EP320. GRANDE HÉTÉROGÉNÉITÉ GÉNÉTIQUE ET NOUVEAU LOCUS POUR LA SCOLIOSE IDIOPATHIQUE

P. Edery (1, 2), P. Margaritte-Jeannin (3), B. Biot (4), A. Labalme (1), J.-C. Bernard (4), J. Chastang (1), B. Kassaï (5), F. Clerget-Darpoux (3) (1) Hospices Civils de Lyon, Service de Cytogénétique Constitutionnelle, Bron, France ; (2) Université Lyonl, France ; (3) Unité d'Épidémiologie Génétique, INSERM U 535, Villejuif, France ; (4) Centre de Réadaptation Fonctionnelle des Massues, Lyon, France; (5) Hospices Civils de Lyon, Centre d'Investigation Clinique (CIC), Bron, France Contact: patrick.edery@chu-lyon.fr

La scoliose idiopathique est une pathologie de la statique rachidienne dont les causes sont inconnues à ce jour, caractérisée par une déviation de la colonne dans le plan frontal (angle de Cobb) d'au moins $10^{\circ}$ et par une rotation vertébrale. La prévalence de cette affection est de 1,5-3\% dans la population générale. Ses conséquences fonctionnelles, esthétiques et sociales sont parfois majeures. Un petit nombre d'analyses de liaison génétique suggère la présence de quelques localisations possibles sur le génome, mais aucune n'a été répliquée de façon convaincante à ce jour. De plus, un polymorphisme du gène $\mathrm{CHD} 7$, impliqué dans le syndrome CHARGE, a été considéré comme prédisposant à la scoliose idiopathique. Toutefois, aucun gène causal n'a été clairement identifié à ce jour. L'étude présentée ici repose sur l'hypothèse de l'existence, à côté du vaste groupe des formes multifactorielles de scoliose idiopathique, d'un groupe restreint de formes «monogéniques » dans lesquelles la maladie est héritée selon un mode dominant. Nous avons donc collecté des familles multiplex de scoliose idiopathique grâce au recrutement du Centre de Réadaptation des Massues, Lyon, France, parmi lesquelles nous avons sélectionné 6 grandes familles multiplex compatibles avec une hérédité dominante. Le critère d'inclusion de la scoliose idiopathique était un angle de Cobb supérieur à $15^{\circ}$, pour limiter le risque de phénocopies (prévalence des scolioses supérieures a $15^{\circ}$ de l'ordre de $1 \%$ ). Nous avons ensuite effectué un tour du génome avec un panel de 1000 marqueurs répartis tous les $3,7 \mathrm{cM}$ en moyenne (deCODE genetics ${ }^{\text {(6) }}$ ) dans ces 6 familles, après une étude d'informativité. Dans seulement une de ces grandes familles, la coségrégation de la maladie et des marqueurs est compatible avec la transmission d'une mutation dominante (partage d'un même haplotype par tous les malades et transmetteurs obligatoires). Pour cette famille, cette parfaite coségrégation est observée en deux régions du génome (5q13-q14 et 3q11-q13) avec un lod score de 2.70. La probabilité d'avoir obtenu ces deux pics en absence de liaison est $<5 \%$ indiquant que le gène muté est situé à une de ces localisations. Dans deux autres grandes familles, nous n'avons trouvé aucune région du génome compatible avec une liaison génétique, suggérant soit une hétérogénéité génétique intrafamiliale soit la présence de phénocopies dans ces familles. De plus, dans chacune des 6 familles étudiées ici, nous avons exclu une compatibilité de liaison avec les autres régions impliquées à ce jour dans la scoliose idiopathique : 19p13.3, 17p11.2, 9q34, 17q25, 18q et $8 \mathrm{q}$ (gène $\mathrm{CHD} 7$ ), suggérant des résultats faux positifs ou une importante hétérogénéité génétique.

Mots-clés : scoliose idiopathique, maladie multifactorielle, formes monogéniques dominantes.

EP321. GENERISK : UN LOGICIEL D'ESTIMATION DES RISQUES POUR DES MALADIES GÉNÉTIQUES À PARTIR DE DONNÉES FAMILIALES

B. Bonaìti $(1,2)$, C. Bonaïti-Pellié $(2,3)$

(1) INRA-GABI, Jouy-en-Josas, France ; (2) INSERM, U535, Villejuif, France

Contact : catherine.bonaiti@inserm.fr

Dans les maladies génétiques à âge de début variable ou dans les formes héréditaires de maladies communes comme le cancer, la prise en charge des personnes porteuses d'une mutation nécessite d'avoir une estimation précise des risques cumulés en fonction de l'âge (fonction de pénétrance), en particulier pour définir des stratégies de prévention. Ces personnes étant le plus souvent diagnostiquées dans des familles recensées sur des critères complexes, et en particulier sur la présence de plusieurs personnes atteintes, les échantillons d'individus porteurs dont on dispose sont bien entendu biaisés. Il est donc crucial, pour obtenir des estimations fiables, de corriger pour ce biais de recensement. Cette correction peut être effectuée grâce à des méthodes basées sur le maximum de vraisemblance. La GRL (genotype restricted likelihood) a été développée dans le cas où les critères son essentiellement familiaux (Carayol et Bonaïti-Pellié, 2004). Son principe est de maximiser, pour chaque famille, la probabilité des génotypes observés conditionnellement aux phénotypes de tous les membres de cette famille, et au génotype de l'individu index (premier individu testé dans la famille) dont le génotype est toujours muté. Cette méthode s'applique préférentiellement aux formes héréditaires de maladies communes. La PEL (Proband's phenotype exclusion likelihood) a été développée dans le cas où les familles ont été recensées indépendamment de l'histoire familiale et s'applique essentiellement aux maladies héréditaires à âge de début variable (Alarcon et Bonaîti-Pellié, 2009). Le logiciel GENERISK permet l'utilisation de ces deux méthodes. La GRL et la PEL ont été généralisées de manière à prendre en compte un éventuel effet de l'origine parentale de la mutation et un effet pléiotropique de cette mutation, c'est-à-dire la possibilité de plusieurs maladies différentes (par exemple des tumeurs de localisations diverses) chez les individus porteurs. Nous présentons les principes des méthodes, leurs applications possibles et les précautions à prendre dans l'utilisation du logiciel. Une illustration de GENERISK sur l'estimation du risque de différentes tumeurs dans le syndrome Lynch/ HNPCC sera présentée par Valérie Bonadona au cours de ces Assises. Carayol J, Bonaîti-Pellié C. Estimating penetrance from family data using a retrospective likelihood when ascertainment depends on genotype and age of onset. Genet Epidemiol $2004 ; 27$ : 109-17. Alarcon F, Bourgain C, Gauthier-Villars M, Planté-Bordeneuve V, Stoppa-Lyonnet D, BonaîtiPellié C. PEL : An unbiased method for estimating age dependent genetic disease risk from pedigree data unselected for family history. Genet Epidemiol $2009: 33: 379-85$.

Mots-clés : estimation, risque, prédisposition.

\section{Maladies monogéniques - de la clinique aux gènes}

DP322. ÉTUDE MOLÉCULAIRE DE 10 FAMILLES NORD-AFRICAINES DE DÉFICIT EN CLASSE II DU SYSTÈME HLA

R. M'rad (1, 3), F. Mellouli (2), M. Trabelsi (1, 3), I. Chelly (1), H. Jilani (1), L. Euchi (1), M. Chaabouni (1, 3), I. Ouertani (1), M. Bejaoui (2), F. Maazoul (1), H. Chaabouni $(1,3)$

(1) Service des maladies congénitales et héréditaires, EPS Charles Nicolle, Tunis, Tunisie: (2) Centre de greffe de Moelle, Bab Saadoun, Tunis, Tunisie; (3) Laboratoire de génétique humaine, Faculté de Médecine de Tunis, Tunis, Tunisie

Contact : rmrad@yahoo.com

Le défaut d'expression des molécules HLA de classe II (MCHII) est responsable d'un déficit immunitaire combiné et sévère appelé<syndrome des lymphocytes nus $>$. C'est une génopathie, rare au cours de laquelle les infections notamment digestives et pulmonaires apparaissent tôt dans la vie Sur le plan biologique il est caractérisé par une absence de la réponse cellulaire et humorale à une stimulation antigénique exogène. Sur le plan moléculaire, il est secondaire à un défaut d'expression d'un des quatre facteurs majeurs de la régulation transcriptionnelle de l'expression du MCHII : CIITA, RFXANK, RFX5, et RFXAP. Le gène RFXANK code pour une sous unité du complexe RFX impliquée dans la régulation transcriptionnelle du système MCHII. Une délétion de $26 \mathrm{pb}$ (752delG-25) a été décrite avec une fréquence élevée. Un effet fondateur de cette mutation dans la population nord africaine a été mis en évidence. Dans ce travail, nous rapportons l'étude moléculaire de 8 familles Tunisiennes et 2 autres Libyennes chez qui le diagnostic de déficit en MHC class II est fortement suspect. Le cas index n'était pas disponible chez $2 / 10$ familles Chez les 8 familles où le cas index est disponible, la délétion (752delG-25) du gène RFXANK a été retrouvée à l'état homozygote chez le malade et hétérozygote chez ces parents dans $5 / 8$ cas. Chez les deux familles où le cas index est décédé, 752 delG-25 est retrouvée à l'état hétérozygote chez les deux parents. Cette étude montre qu au moins $75 \%$ des patients tunisiens sont porteurs de cette anomalie. Ces résultats confirment l'effet fondateur décrit dans la population nord africaine et permettent de proposer un conseil génétique et un diagnostic anténatal fiable dans les familles à risque.

Mots-clés : RFXANK, Afrique du nord, déficit immunitaire.

DP323. ANALYSIS OF TAZ (TAFAZZIN) AND LDB3 (LIM DOMAIN-BINDING3/CYPHER/ZASP) GENES IN LEFT VENTRICULAR NON COMPACTION

E. Villard (1), G. Habib (2), E. Donal (3), J.C. Eicher (4), C. Pascal (5), R. Isnard (1), G. Dilanian (1), M. Komajda (1), P. Charron (1)

(1) AP-HP - Hopital La Pitie-Salpetriere, Paris, France ; (2) AP-HM Hopital de la Timone, Marseille, France; (3) CHU de Rennes - Hopital de Pontchaillou, Rennes, France ; (4) Centre Hospitalier Universitaire de Dijon, Dijon, France ; (5) CHU de Nantes - Hopital Guillaume et Rene Laennec, Nantes, France

Contact : philippe.charron@psl.aphp.fr 
Background: Left ventricular non compaction (LVNC) is a recently identified cardiomyopathy, characterized by an excessively prominent trabecular meshwork and deep intertrabecular recesses. Some genes have been described as responsible for LVNC, including TAZ and LDB3, but the precise prevalence of these genes and the impact of mutation screening in clinical practice are poorly understood, Objective: To assess the prevalence of mutations in TAZ (tafazzin, Xq28) and LDB3 (LIM domain-binding3/Cypher/ZASP, 10q23.2) genes in a large cohort of patients with LVNC, whatever the familial context. Methods : DNA was extracted from a population of 59 consecutive patients with a definitive diagnosis of LVNC (Echo core lab), from the French registry of LVNC. Direct sequencing of exons and intron-exon boundaries was performed with ABI Prism 3100 Genetic Analyzer (Applied Biosystems). The suspected mutations were tested in a control population ( $>240$ chromosomes); segregetion within the families were analysed when available; evolutive conservation among various animal species were analysed by apropriate software. Results: We identified two new missense mutations in the TAZ gene (Phe128Ser and Met155Val) in two index male patients. No mutation was observed in the LDB3 gene, but two new genetic polymorphisms. The prevalence of TAZ mutations was $3 \%(2 / 59)$ and $0 \%$ for LDB3. Conclusion: Mutations in TAZ gene were not unfrequent in LVNC whereas no mutation was observed in LDB3 gene. These findings may have impact for LVNC mutation screening strategy in clinical practice, and also for genetic counselling as TAZ mutations are associated with $\mathrm{X}$-linked inheritance.

Mots-clés : cardiomyopathie, mutation.

P324. SYNDROME DE BARTH : NOUVELLES MUTATIONS DU GÈNE TAZ ET VALIDATION DES CRITÈRES DIAGNOSTIQUES V. Koubi (1), A. Chabli (1), M. Jambou (1), N. Brahimi (1), R. Tourraine (2), J.L. Stephan (2), G. Viot (3), S. Lyonnet (1), P. De Lonlay (1), M. Willems (1), J. Steffann (1), J.P. Bonnefont (1), A. Munnich (1), A. Rötig (1), A.S. Lebre (1)

(1) Université Paris Descartes, Hôpital Necker-Enfants Malades et Inserm U781, Départements de Génétique, des Maladies Métaboliques et de Biochimie B, Paris, France ; (2) Université de Saint Étienne, Hôpital Nord, CHU Saint-Étienne, Services de génétique et de pédiatrie, Saint-Étienne, France; (3) Université Paris Descartes, Hôpital Cochin, Département de Génétique, Paris, France

Contact : valeriekoubi@yahoo.fr

Objectifs : Le syndrome de Barth (BTHS, MIM 302060) est une pathologie récessive liée à l'X, caractérisée par l'association d'une cardiomyopathie précoce, une myopathie des muscles squelettiques, un retard de croissance et d'une neutropénie cyclique. Depuis l'identification du gène TAZ (NM_ 000116, Xq28) dans le BTHS (en 1996), plus de 40 mutations ont été décrites (HGMD). Le gène TAZ code pour la protéine tafazzine, qui, lorsqu'elle est mutée engendre des anomalies mitochondriales via une altération dans le remodelage des cardiolipines et des phosphatidylglycerol membranaires. Les anomalies des cardiolipines sont mises en évidence par dosage en chromatographie liquide haute performance couplée à la spectrométrie de masse (hPLCSM). Chez les patients BTHS, il existe une diminution des cardiolipines (CL), une augmentation des monolysocardiolipines (MLCL) et une élévation spécifique du ratio MLCL/CL. La recherche de mutation du gène TAZ est alors effectuée par séquençage direct des 11 exons. À ce jour, aucune corrélation phénotype-génotype n'a pu être clairement établie et il existe de grandes variations phénotypiques inter et intra-familiales. Méthodes : Nous avons collecté des échantillons tissulaires (peau, cœur, foie) de 50 patients de sexe masculin avec suspicion de syndrome de Barth afin de réaliser une analyse biochimique des cardiolipines par hPLC-SM. Résultats : Parmi les 50 patients étudiés, 8 ont été confirmés comme porteurs d'un syndrome de Barth. La comparaison des tableaux clinico-biologiques chez ces malades nous a permis de mettre en évidence la constante association d'une cardiomyopathie dilatée. d'une neutropénie cyclique et d'une anomalie du profil des cardiolipines chez des garçons âgés de moins de 6 mois. Le retard de croissance, l'hypotonie, l'atrophie musculaire, les difficultés alimentaires et les infections répétées sont fréquents. Le séquençage du gène TAZ a mis en évidence 8 mutations distinctes pour chacun des patients : 4 substitutions (exons 3,4,8), 1 substitutioninsertion (exon 2), 1 insertion (exon 9) et 2 délétions de plusieurs exons. Parmi celles-ci, 2 mutations ont déjà été rapportées dans la littérature mais aucune des délétions retrouvées n'a été décrite à ce jour. Au total, nous avons donc mis en évidence 6 nouvelles mutations dans le gène TAZ responsables d'un syndrome de Barth. En conclusion, l'association chez un nourrisson de sexe masculin d'une cardiomyopathie dilatée et d'une neutropénie cyclique doit faire suspecter un syndrome de Barth. Une anomalie du profil des cardiolipines doit être recherchée sur une biopsie de peau. L'élévation du ratio et l'identification d'une mutation permettra de proposer aux patients un conseil génétique ainsi qu'un éventuel diagnostic anténatal.

Mots-clés : syndrome de Barth, cardiomyopathie, gène TAZ.

TP325. GÈNES DU DESMOSOME ET DYSPLASIE VENTRICULAIRE DROITE ARYTHMOGENE : SPECTRE DES MUTATIONS ET IMPLICATIONS CLINIQUES

V. Fressart (1), G. Duthoit (2), E. Donal (3), V. Probst (4), J.C. Deharo (5), P. Chevalier (6), D. Klug (7), O. Dubourg (8), E. Delacretaz (9), P. Cosnay (10), P. Scanu (11), D. Keller (12), F. Hidden-Lucet (2), C. Coirault (13), Y. Lecarpentier (13), B. Hainque (1), F. Simon (1), V. Bessirard (14), J.L. Hébert (13), G. Fontaine (2), R. Frank (2), P. Charron (14)

(1) Service de Biochimie Métabolique, Hôpital Pitié-Salpêtrière, Paris, France ; (2) Département de Cardiologie, Hôpital Pitié-Salpêtrière Paris, France; (3) Service de Cardiologie, Hôpital Pontchaillou, Rennes, France; (4) Service de Cardiologie, Hôpital Laennec, Nantes, France; (5) Service de Cardiologie, Hôpital La Timone, Marseille, France; (6) Service de Cardiologie, Hôpital Est, Lyon, France; (7) Service de Cardiologie, Hôpital Cardiologique, Lille, France; (8) Université de Versailles - Saint Quentin, Hôpital Ambroise Paré, Boulogne, France ; (9) Service de Cardiologie, Hôpital de l'lle, Berne, Suisse; (10) Service de Cardiologie, CHU Tours, Tours, France: (11) Service de Cardiologie, Hôpital Cote de Nacre, Caen, France: (12) Service de Cardiologie, Hôpital universitaire de Bâle, Bâle, Suisse; (13) Service d'Explorations Fonctionnelles Cardiorespiratoires, Hôpital de Bicêtre, Paris, France ; (14) Département de Génétique, AP-HP, UPMC Université Paris 6, Paris, France

Contact : vero.fressart@psl.aphp.fr

Introduction : La dysplasie ventriculaire droite arythmogène (DVDA) est une cardiomyopathie héréditaire (prévalence 1/2000 environ) responsable de mort subite, en particulier chez des sujets jeunes et volontiers sportifs. Cinq gènes codant des protéines du desmosome ont été impliqués dans la genèse de cette pathologie : JUP, DSP, PKP2, DSG2 et DSC2. Nous avons réalisé l'analyse systématique de ces cinq gènes chez des patients atteints de DVDA avérée afin de connaître la fréquence de mutation des différents gènes testés et d'évaluer l'impact potentiel du génotype sur le phénotype. Matériels et Résultats : L'analyse a été réalisée par séquençage direct de ces 5 gènes chez 135 probands indépendants répondant aux critères de l'International Task Force for ARVD/C (arrhythmogenic right ventricular dysplasia/cardiomyopathy). Nous avons identifié chez 62 patients ( $46 \%$ ), 41 différentes mutations causales dont 29 nouvelles mutations. De plus, des variants dits de signification inconnue (VSI) ont été objectivés chez 9 autres patients (7\%). Les mutations causales sont distribuées comme suit : $31 \%$ dans PKP2, $10 \%$ dans DSG2, $4.5 \%$ dans DSP, $1,5 \%$ dans DSC2 et $0 \%$ dans JUP. Des mutations privées ont été retrouvées dans $39 \%$ des cas mutés ( 24 des patients mutés sur 62 ). La présence d'une mutation était liée significativement à lâge du proband, à la présence de potentiels tardifs, au caractère symptomatique du patient, à l'atteinte ventriculaire gauche, à l'atteinte diffuse du ventricule droit, mais pas au contexte familial. Les mutations dans DSG2 étaient associées à une diminution de la Fraction d'Éjection (FE) du ventricule gauche (VG) : $50 \%$ des patients porteurs de mutation DSG2 avaient une FEVG diminuée contre $33 \%$ des patients porteurs de mutation DSP et seulement $10 \%$ des patients porteurs de mutation PKP2 $(\mathrm{p}=0,008)$. Enfin, la présence de deux mutations, objectivée chez $4 \%$ des patients ( $8 \%$ en tenant compte des VSI) était associée à un risque supérieur de survenue de mort subite : $40 \%$ chez les porteurs de deux mutations $(\mathrm{N}=5)$ contre $5 \%$ chez les porteurs d'une seule mutation identifiée $(N=57)(p=0,047)$. Conclusions : Il s'agit de la première analyse exhaustive des cinq gènes codant les protéines du desmosome impliqués dans la DVDA. Nos résultats suggèrent la possibilité d'utiliser le test génétique comme une aide au diagnostic de cette maladie, avec la perspective d'une aide au diagnostic des cas douteux. La découverte d'une mutation dans DSG2 ou d'une double mutation semble être associée respectivement à un risque évolutif accru vers l'insuffisance cardiaque ou la survenue de mort subite et revêtrait donc un caractère pronostique.

Mots-clés : dysplasie ventriculaire droite arythmogène, desmosome, Plakophilline2, Desmocolline2, Desmogléine2, Plako.

mP326. À PROPOS DE DEUX VARIANTS R148W ET R176W DE KCNH2 : MUTATIONS RESPONSABLES DE SYNDROME DE QT LONG OU POLYMORPHISMES RARES

V. Fressart (1, 2), C. Ormand (1), I. Magnin Poull (3), F. Rousselet (4), J.M. Lupoglazoff $(2,5,6)$, B. Hainque $(1,2)$, P. Charron $(2,5,7)$, M. Berthet (2), P. Guicheney (2), I. Denjoy $(2,5,8)$

(1) Service de Biochimie Métabolique, Hôpital Pitié-Salpêtrière (AP-HP), Paris, France; (2) INSERM U956, Hôpital Pitié-Salpêtrière (AP-HP), Paris, France; (3) Service de Cardiologie, CHU-Nancy, Nancy, France; 
(4) Département de Génétique, CHU-Nancy, Nancy, France; (5) Centre de Référence pour les Maladies Cardiaques Héréditaires, Paris, France; (6) Service de Cardiologie Infantile, Hôpital Robert-Debré, Paris, France ; (7) Département de Génétique, Hôpital Pitié-Salpêtrière (AP-HP), Paris, France ; (8) Service de Cardiologie, Hôpital Lariboisière (APHP), Paris, France

Contact : vero.fressart@psl.aphp.fr

Le syndrome du QT long congénital (QTL) se caractérise à l'électrocardiogramme (ECG) de surface par un allongement de l'intervalle QT associé à un risque de troubles du rythme ventriculaire graves. Le syndrome du QTL est cliniquement hétérogène: QTc allongé isolé, bradycardie sinusale, lipothymies, syncopes et morts subites qui en font toute la gravité. Le diagnostic est porté classiquement chez des sujets jeunes. La transmission est habituellement autosomique dominante. En plus des formes dites congénitales, il existe des formes dites acquises, médicamenteuses ou liées à des désordres hydro-électrolytiques. Le syndrome du QTL congénital se caractérise par une grande hétérogénéité génétique, plus de 10 gènes sont impliqués dans ce syndrome. Parmi eux, le gène $\mathrm{KCNH} 2$ codant un canal potassique, est le siège de 40 à $50 \%$ des mutations causales de QTL. En l'absence d'études électrophysiologiques, il est difficile de déterminer si certains nouveaux variants faux sens sont des mutations ou des polymorphismes. Ils sont alors classés comme variant de signification inconnu (VSI). Ce travail a pour but de montrer les limites des démarches classiques permettant d'évaluer le caractère causal de variants. Deux variants, p.Arg $148 \mathrm{Trp}, \mathrm{c} .422 \mathrm{C}>\mathrm{T}$ et p.Arg $176 \mathrm{Trp}, \mathrm{c} .526 \mathrm{C}>\mathrm{T}$ ont été identifiés dans le gène $\mathrm{KCNH} 2$, chez 4 et 2 familles, en l'absence d'autres mutations dans les gènes fréquemment mutés dans le syndrome du QTL. Ils sont localisés dans le domaine extracellulaire entre les segments I et II de la protéine. L'analyse du variant par logiciels de prédictions, l'étude de la ségrégation familiale (recrutement des apparentés, évaluation de leur atteinte clinique, confrontation phénotype génotype) la recherche du variant chez des contrôles sont des aides précieuses à la décision. Le plus souvent, l'analyse de tous ces critères permet de conclure quant au caractère pathogène du variant. Cependant, dans certains cas, la difficulté persiste et le choix entre polymorphisme rare ou mutation ne peut être fait. Les deux variants décrits ont aussi bien des caractéristiques de polymorphisme que de mutation d'après les logiciels de prédiction. L'analyse de la ségrégation familiale ne permet pas davantage de trancher sur la nature causale du variant car la durée de l'intervalle QTc varie de normale à clairement prolongée chez les porteurs. Enfin, l'absence des deux variants chez 100 contrôles permet de les éliminer en tant que polymorphisme fréquent, mais pas de trancher entre polymorphisme rare et mutation. Les procédures habituelles permettant la validation d'un variant comme polymorphisme ou mutation peuvent être insuffisantes. Les résultats suggèrent que ce pourrait être des variants rares à faible effet associés à une autre mutation non encore identifiée chez les sujets les plus atteints des familles. L'analyse fonctionnelle de ces deux variants après expression in vitro serait d'une aide appréciable pour statuer sur leur caractère causal.

Mots-clés : syndrome de QT long, KCNH2.

IP327. EXPLORATION MOLÉCULAIRE D'UNE COHORTE DE 100 PATIENTS FRANÇAIS PORTEURS DE CARDIOMYOPATHIE DILATÉE

G. Millat (1), H. Crehalet (1), V. Chanavat (1), S. Julia (2), P. Bouvagnet (3), R. Rousson (1)

(1) Laboratoire de Cardiogénétique Moléculaire, Centre de Biologie et Pathologie EST, Bron, France; (2) Service de Génétique Médicale, Hôpital Purpan, CHU Toulouse, Toulouse, France; (3) Service de Cardiologie C, Hôpital CardioVasculaire et Pneumologique L.-Pradel, Bron, France

Contact : gilles.millat@chu-lyon.fr

La cardiomyopathie dilatée (CMD) est une maladie du muscle cardiaque, caractérisée par une dilatation ventriculaire et une altération de la fonction systolique. Les patients présentent une insuffisance cardiaque, une arythmie et un risque de mort prématurée. La prévalence de la cardiomyopathie dilatée est de $1 / 2500$ individus. La cardiomyopathie dilatée familiale représenterait entre 20 à $40 \%$ des cardiomyopathies dilatées. Cette forme familiale est principalement due à des mutations des gènes codant des protéines du cytosquelette et du sarcomère des myocytes cardiaques. À ce jour, plus de 25 gènes morbides sont impliqués dans cette pathologie. Les différentes études rapportées à ce jour suggèrent que les gènes les plus fréquemment mutés sont les gènes MYH7, LMNA et TNNT2. Une exploration moléculaire de ces gènes a donc été initiée à partir de l'ADN génomique de 100 patients français présentant une cardiomyopathie dilatée. Après amplification des exons et des jonctions exons-introns de chacun de ces 3 gènes, les amplicons ont été analysées soit par DHPLC/ séquençage (gènes MYH7 et TNNT2), soit par HRM/séquençage (gène
LMNA). Cette étude a permis d'identifier une mutation dans 16 familles CMD (16\%). Parmi les 15 mutations identifiées, 8 sont nouvelles et 7 ont été précédemment rapportées dans la littérature. Aucune de ces mutations n'a été identifiée après analyse de 200 chromosomes témoins. Plus de la moitié des mutations ont été localisées dans le gène LMNA qui représente donc le gène le plus fréquemment muté ( $9 \%$ des patients $C M D)$ au sein de notre cohorte. Les mutations LMNA sont 5 mutations faux-sens (p.Leu92Phe ; p.Glu161Lys ; p.Ala287fs; p.Glu317Lys; p.Arg377His et p.Gly523Arg), une insertion de $24 \mathrm{pb}$ (p.ins128_129insRVTLISSR), une insertion de $1 \mathrm{pb}$ (p.Ala287fs) et une mutation d'épissage (IVS7+1 G>A). Les 7 autres mutations identifiées sont 4 mutations TNNT2 ( $4,9 \%$ des patients CMD), et 3 mutations faux-sens MYH7 (3,9\% des patients CMD). Les mutants faux-sens MYH7 (p.Val1044Ala; p.1101-1104 delGSQL ; p.Ala1263Glu) sont localisés au sein de la partie tige de la protéine MYH7. Les fréquences obtenues sont similaires à celles rapportées dans la littérature. Au vu du grand nombre de gènes morbides impliqués, il est probable qu'une étude moléculaire plus exhaustive (gènes MYPN, MYBPC3, TNNI3, ANKRD1, RBM20...) aurait permis de déceler une anomalie moléculaire chez un plus grand nombre de patients. L'identification soit de nouvelles mutations dans des gènes connus soit de nouveaux gènes morbides est cruciale pour mieux définir les corrélations phénotype/génotype et ainsi mieux appréhender la physiopathologie de cette maladie. Cette identification est également nécessaire pour améliorer la prise en charge en thérapeutique des malades et permettre la mise en place d'un conseil génétique adapté pour les différents apparentés.

\section{IP328. UNE MUTATION DU GENE KCNQ1 RESPONSABLE DU SYNDROME DU QT LONG ET DE FIBRILLATION ATRIALE FAMILIALE}

S. Duchatelet (1), D.C. Bartos (2), D. Klug (3), J.M. Lupoglazoff (1, 4, 5), I. Denjoy (1, 5, 6), M. Berthet (1), V. Fressart (1, 7), B.P. Delisle (2), P. Guicheney (1)

(1) Inserm U956, Hôpital Pitié-Salpêtrière, Paris, France ; (2) University of Kentucky, Lexington, KY, USA ; (3) Service de cardiologie A, Hôpital cardiologique, Lille, France; (4) Service de cardiologie Infantile, Hôpital Robert Debré, Paris, France ; (5) Centre de Référence pour les Maladies Cardiaques Héréditaires, Paris, France; (6) Service de cardiologie, Hôpital Lariboisière, Paris, France; (7) Service de Biochimie Métabolique, Hôpital Pitié-Salpêtrière, Paris, France

Contact : pascale.guicheney@upmc.fr

Le syndrome du QT long congénital (SQTL) est une maladie cardiaque héréditaire rare qui se caractérise par un allongement de la durée de l'intervalle QT sur l'électrocardiogramme et un risque de syncope et de mort subite, le plus souvent de transmission autosomique dominante. De nombreuses mutations ont été identifiées dans le gène KCNQ1 codant une sous-unité du canal potassique voltage-dépendant à l'origine du courant sortant repolarisant IKS. La plupart d'entre elles sont responsables du SQTL et sont associées à une réduction du courant potassique sortant IKS. Quelques rares mutations associées à une activation d'IKS sont à l'origine du syndrome du QT court ou de fibrillation atriale familiale (FA). Nous avons identifié la mutation p.Arg231Cys dans KCNQ1 dans 6 familles, 5 d'entre elles présentaient des formes sévères de syndrome du QT long (QTc $>500 \mathrm{~ms}$ ). Dans la 6ème famille, 4 des 5 porteurs de la mutation, présentaient une FA ayant débuté entre les âges de 15 et 33 ans, associée ou non à un allongement modeste de l'intervalle QT. Nous avons exclu dans cette famille la présence d'une autre mutation dans la plupart des gènes décrits comme responsables de FA (KCNQ1, KCNH2, SCN5A, KCNE2, KCNE3, KCNE5, KCNA5, KCNJ2, NPPA, GJA5). L'analyse fonctionnelle de la mutation p.Arg231Cys, après expression hétérologue, a montré un comportement inhabituel du canal muté. En effet, une diminution du courant potassique est observée pour des potentiels de membrane positifs, et une augmentation du courant potassique aux potentiels négatifs. Ces résultats ne permettent pas d'expliquer les différents phénotypes observés dans ces familles. Cette variabilité phénotypique suggère l'implication d'autres facteurs génétiques dans l'expression clinique de la maladie. Nous avons recherché l'ensemble des SNPs décrits comme ayant un effet protecteur ou à risque sur la FA d'après les études de gènes candidats et les analyses d'association génome-entier. Nous avons sélectionné 12 polymorphismes dans 10 gènes $(\mathrm{KCNH} 2, \mathrm{KCNE} 1, \mathrm{KCNE} 4, \mathrm{KCNE} 5, \mathrm{SCN} 5 \mathrm{~A}$, GJA5, SLN, IL10, PITX2, ZFHX3) et nous les avons génotypés dans ces 6 familles. La plupart des SNPs étudiés sont retrouvés dans les familles SQTL et la famille FA. Dans la famille FA, l'allèle T protecteur du SNP (rs17003955, c.97C > T, p.Pro33Ser) du gène KCNE5 n'est présent que chez le porteur de la mutation p.Arg231Cys n'ayant pas développé de FA. KCNE5 code une sous-unité régulatrice de KCNQ1 qui pourrait donc moduler les conséquences fonctionnelles de la mutation de KCNQ1. Des études fonctionnelles vont être réalisées afin de déterminer l'effet de la 
sous-unité codée par KCNE5 et de ce polymorphisme sur les conséquences fonctionnelles de la mutation p.Arg231Cys. Néanmoins, d'autres patients dans les familles SQTL sont porteurs de l'allèle C du SNP rs17003955 comme ceux qui présentent une FA; ceci suggère que d'autres facteurs génétiques non encore identifiés contribuent à la variabilité phénotypique associée à cette mutation.

Mots-clés : syndrome du QT long, fibrillation atriale, KCNQ1.

EP329. SPORADIC ARRHYTHMOGENIC RIGHT VENTRICULAR CARDIOMYOPATHY DUE TO A DE NOVO MUTATION E. Gandjbakhch (1, 2), V. Fressart (3), G. Bertaux (4), L. Faivre (5), F. Simon (3), R. Frank (1), G. Fontaine (1), E. Villard (2), C. Coirault (6), B. Hainque $(3,6)$, P. Charron $(1,2,7)$

(I) Département de Cardiologie, Hôpital Pitié-Salpêtrière, AP-HP, Paris, France; (2) INSERM, UMR-S956, AP-HP, UPMC Université Paris 6, Paris, France; (3) Service de Biochimie, Unité de Cardiogénétique et Myogénétique, Hôpital Pitié-Salpêtrière, AP-HP, Paris, France; (4) Centre de Cardiologie, Centre Hospitalier Universitaire, Dijon, France, (5) Centre de Génétique, Centre Hospitalier Universitaire, Dijon, France ; (6) INSERM U582, Hôpital Pitié-Salpêtrière, AP-HP, Paris, France ; (7) Département de Génétique, Hôpital Pitié-Salpêtrière, AP-HP, Paris, France

Contact : estelle.gandjbakhch@upmc.fr

We report the case of a 41-year-old man with a diagnosis of sporadic arrhythmogenic right ventricular cardiomyopathy (ARVC). The electrocardiogram displayed extensive $\mathrm{T}$ waves inversion in précordial leads. The 24-hour ECG monitoring documented more than 500 polymorphic ventricular ectopies. Signal-averaged ECG with $40 \mathrm{~Hz}$ filter showed late potentials. The echocardiogram showed right ventricular (RV) abnormalities with global mild RV dilatation, wall motion abnormalities localised in the inferior wall and the apex, and excessive trabeculations, while left ventricle appeared normal. RV abnormalities were confirmed by cardiac noncontrast cine-MRI. The electrophysiological study easily induced reproducible fast ventricular tachycardia with two different left-bundle-branch block morphologies. The genetic screening of the four desmosomal genes plakophilin-2, desmoplakin, desmoglein-2, desmocollin-2 (that have been shown to be involved in ARVC) identified the heterozygous missense mutation $\mathrm{R} 49 \mathrm{H}$ in the desmoglein-2 gene. This mutation is located in the highly conserved cleavage motif $\mathrm{RXK} / \mathrm{RR}$ that is recognised by pro-protein convertases and is thus predicted to prevent efficient pro-desmoglein-2 maturation. The mutation was absent in both parents, and we demonstrated that it was a de novo mutation. To the best of our knowledge, this is the first description of a de novo mutation in ARVC. Appearance of a de novo mutation in the desmoglein- 2 gene (that is an essential component of desmosome that mediates cell-to-cell adhesion) provides compelling genetic evidence for the involvement of this gene in ARVC. The recognition of de novo mutations has important implications, including for clinical practice, since individuals with sporadic ARVC caused by a de novo mutation can transmit the disease gene to $50 \%$ of their offspring. This suggests that the benefit of molecular genetics can be extended to sporadic ARVC, and may improve genetic counselling.

Mots-clés : cardiomyopathy, ARVC, genetics

\section{DP330. KATP CHANNELS AND EARLY REPOLARIZATION SYNDROME}

S. Chatel (1), M. Haïssaguerre (2), F. Sacher (2), R. Weerasooriya (2), V. Probst (1, 3), G. Loussouarn (1), M. Horlitz (5), R. Liersch (5), E. Schulze-Bahr (6), A. Wilde (7), S. Kääb (8), J. Koster (4), Y. Rudy (4), F. Kyndt (1), H. Le Marec (1, 3), J.J. Schott (1)

(1) INSERM UMR915, CNRS ERL3147 l'institut du thorax, Nantes, France ; (2) Université de Bordeaux, Hôpital Haut-Lévêque, BordeauxPessac, France; (3) Service de Cardiologie, L'institut du Thorax, CHU de Nantes, Nantes; (4) Washington University School of Medicine, St. Louis, Missouri, USA ; (5) Klinikum Wuppertal, Germany ; (6) Department of Cardiology and Angiology, Hospital of the University of Münster, Münster, Germany; (7) Academic Medical Centre, Amsterdam, The Netherlands; (8) Medizinische Klinik und Poliklinik I Marchioninistraße, Munich, Germany

Contact : stephanie.chatel@nantes.inserm.fr

Early repolarization is a common electrocardiographic finding that is generally considered to be benign and affects 1 to $5 \%$ individuals. Potential arrhythmogenicity has been suggested, but so far clinical evidence was lacking. A recent case-control study reveals that early repolarization is more frequent in case subjects with idiopathic ventricular fibrillation than with idiopathic ventricular fibrillation have a family history of sudden cardiac arrest which suggests the presence of genetic defects. Early repolarization could involve a transmural repolarization gradient across the ventricular wall, caused by an anomaly of epicardium action potential. This anomaly can be due to an increase of current IKATP. ATP-sensitive potassium channel (KATP) are heteromers composed of inwardly rectifying $\mathrm{K}+$ subunits Kir6.0 (Kir6.1 encoded by KCNJ8 or Kir6.2 encoded by KCNJ11) and sulphonylurea receptors SUR (SUR1 encoded by ABCC8 or SUR2 encoded by ABCC9). A candidate gene approach by direct sequencing was performed in a cohort of 96 patients. We first describe a variant in $\mathrm{KCNJ} 8$ (c. $1265 \mathrm{C}>\mathrm{T}$ p.S $422 \mathrm{~L}$ ) in a young female presenting with multiple episodes of idiopathic ventricular fibrillation associated with very dramatic changes in repolarization pattern. In a second step, we sequenced other cardiac KATP channel subunits and identified 5 rare variants in ABCC 9 in 5 probands: 4 missense variants and a nucleotide substitution in a splice-site. Enrollment of these patient's family and functional analysis are in progress. Several physiopathological hypothesis (membrane trafficking increased, ATP sensitivity decreased...) are going to be tested in order to investigate the electrophysiological consequences and establish a pathogenetic link between phenotype and genotype. The identification of 6 patients among $96(6.25 \%)$ with a variant in different KATP channel subunits reinforces the implication of ATP-sensitive channels in early repolarization syndrome.

Mots-clés : syndrome de repolarisation précoce, canaux Katp, approche gène candidat.

DP331. ÉTUDE GÉNÉTIQUE DES FORMES PÉDIATRIQUES DU SYNDROME DE WOLFF PARKINSON WHITE : ÉVIDENCE D'UNE HÉTÉROGÉNÉITÉ GÉNÉTIQUE DANS LA POPULATION TUNISIENNE

S. Nouira (1), F, Ouarda (2), C. Charfeddine (1), I. Arfa (1), H. Ouragini (1), F. Abid (2), S. Abdelhak (1)

(1) Unité de Recherche UR04/SP03 « Exploration Moléculaire des Maladies Orphelines d'Origine Génétique », Institut Pasteur de Tunis, Tunisie ; (2) Service de cardiologie pédiatrique de l'Hôpital de la Rabta, Tunis, Tunisie

Contact : nouira_sonia@yahoo.fr

Le Wolff Parkinson White (WPW, MIM 194200) est une anomalie congénitale rare avec une prévalence estimée à $1 / 1000$. Cette maladie est caractérisée par l'existence d'une voie de conduction supplémentaire entre oreillette et ventricule (Faisceau de Kent) se traduisant sur le plan électrophysiologique par une pré-excitation ventriculaire et une tachycardie. Le WPW peut se présenter sous une forme isolée ou associée à une cardiomyopathie héréditaire (CMH). Ces deux formes cliniques sont transmises suivant le mode autosomique dominant. Des mutations dans le gène PRKAG2 localisé dans la région chromosomique $7 \mathrm{q} 36$ seraient responsables des formes familiales de WPW. Ce gène code pour la sous unité gamma 2 de la protéine kinase AMP dépendante qui joue un rôle majeur dans le maintien de l'équilibre cellulaire du rapport AMP/ATP par la régulation des enzymes qui déterminent le flux d'énergie dans la cellule. L'implication des gènes PRKAG2 et NKX2-5 a été étudiée chez 3 familles tunisiennes présentant 11 individus atteints du WPW. L'investigation clinique montre une large variabilité phénotypique. L'analyse mutationnelle par séquençage direct montre qu'à l'exception de deux polymorphismes, aucune mutation n'a été identifiée dans les parties codantes des gènes PRKAG2 et NKX2-5 chez les familles étudiées. Ces résultats sont en faveur d'une hétérogénéité clinique et génétique du WPW dans la population Tunisienne. Associée à la présence d'individu non pénétrant et l'influence de facteurs environnementaux et épigénétiques, cette hétérogénéité complique l'étude des cardiopathies héréditaires.

Mots-clés : Wolff Parkinson White, PRKAG2, analyse génétique.

IP332. DÉFICIENCE EN GDP-MANNOSE : GLCNAC2-PP-DOLICHOL MANNOSYLTRANSFÉRASE (ALG1, CDGIK) : 8 NOUVEAUX PATIENTS ET 10 NOUVELLES MUTATIONS

T. Dupré $(1,2,5)$, S. Vuillaumier-Barrot $(1,2,5)$, I. Chantret $(2,5)$, H. Sadou Yayé (1), C. Le Bizec (1), A. Afenjar (6), C. Altuzarra (7), C. Barnérias (8), L. Burglen (9), P. De Lonlay (3, 4), F. Feillet (10), S. Napuri (11), G. Matthijs (12), S.E.H. Moore (2, 5), N. Seta $(1,3)$ (1) AP-HP, Hôpital Bichat-Claude Bernard, Biochimie Métabolique et Cellulaire, Paris France ; (2) INSERM U773 CRB3, Paris France : (3) Université Paris-Descartes, Paris, France; (4) AP-HP, Hôpital NeckerEnfants Malades, Département de Pédiatrie, Paris, France ; (5)Université Denis-Diderot, Paris 7. Paris, France ; (6) AP-HP, Service de Neuropédiatrie et Pathologie du Développement, Hôpital Armand-Trousseau, Paris, France ; (7) Service de Pédiatrie, Hôpital Saint Jacques, Besançon, 
France; (8) AP-HP, Service de Neuropédiatrie, and Centre de Référence des Maladies Neuromusculaires, Hôpital Necker-Enfants-malades, Paris, France ; (9) AP-HP, Service de Génétique, Génétique Clinique-Neurogénétique, Hôpital A.-Trousseau, Paris, France; (10) Centre de Référence des Maladies Héréditaires du Métabolisme, CHU Brabois Enfant, Vandauvre-lès-Nancy, France; (11) Explorations Fonctionnelles Neurologiques Hôpital Sud-Rennes, Rennes, France ; (12) Center for Human Genetics, University of Leuven, Belgique

Contact : sandrine.vuillaumier@bch.aphp.fr

Les « Congenital Disorders of glycosylation » de type I (CDG I), constituent un groupe de pathologies héréditaires transmises sur un mode autosomique récessif. Ils sont liées à des défauts d'une des étapes précoces de la synthèse de la partie glycanique des $\mathrm{N}$-glycoprotéines. Les CDG Ik sont dus à une carence en GDP-mannose: GlcNAc2-PP-dolichol mannosyltransferase (MT-1, gène ALG1). À ce jour, seuls quatre patients, avec des présentations cliniques sévères, ont été décrits. Nous présentons ici les données génétiques, biochimiques et cliniques de 8 nouveaux cas de CDG lié à MT-1 : 5 cas français et 3 cas du réseau européen Euroglycanet La biosynthèse des oligosaccharides lipides (OSL) a été explorée dans des fibroblastes de biopsie de peau en culture et l'activité mannosyltransferase MT-1 a été dosée dans les microsomes préparés à partir de ces cellules. Le gène ALG1 (codant pour MT-1) a été séquencé à la fois sur ADN génomique et ADNc. Les fibroblastes en culture issus d'une biopsie de peau des 5 patients français ont été marqués par de la glucosamine tritiée. Ils ont présenté des accumulations de GlcNAc2-PP-dolichol, le deuxième intermédiaire dans la biosynthèse de OSL indiquant un possible déficit de MT-1. Les activités des mannosyltransferases MT-1, MT-2 et MT-3, permettant l lajout respectivement des 3 premiers résidus mannosyl de l'OSL ont été mesurées. Seule l'activité MT-1 a été trouvée déficitaire. Le séquençage du gène ALG1 chez les 8 patients a révélé 12 mutations différentes : 2 mutations connues pour être causales: c.773C $>\mathrm{T}$ (Ser258Leu) et c. $450 \mathrm{C}>\mathrm{G}$ (p.Ser150Arg) et 10 mutations qui n'ont pas été décrites précédemment dont 6 faux sens (c.434G $>$ A (p.Gly145Asp), c.397C $>$ A (p.Pro133Thr), c.826C $>\mathrm{T}$ (p.Arg276Trp), c.1129A $>\mathrm{G}$ (Met377Val), c. $1145 \mathrm{~T}>\mathrm{A}$ (p.Met382Lys), c.1312C $>\mathrm{T}$ (p.Arg438Trp) et 4 mutations d'épissage [c.740G $>\mathrm{T}$, c.740 +5G $>\mathrm{A}$ (p.Ala211_Arg247del)], c. $961+1 \mathrm{G}>\mathrm{C}, \mathrm{c} .1263 \mathrm{G}>\mathrm{A}$ (p.Cys396X). Les nouvelles mutations faux sens sont données comme pathogènes par les logiciels de prédiction phénotypique. Les présentations cliniques sont sévères avec des dysmorphies, une atteinte du SNC et des troubles oculaires fréquents. Ces nouvelles données modifient radicalement la fréquence relative des CDG I dans notre cohorte de patients CDG diagnostiqués en France. En effet, jusqu'à présent, le CDG Ik décrit dans la littérature seulement pour 4 patients dans le monde devient dans notre série le deuxième sous type de CDG I le plus fréquent après le CDG Ia (déficit en phosphomanomutase), à égalité avec le CDG $\mathrm{Ib}$ (déficit en phosphomannose isomérase). Notre stratégie diagnostic s'en trouve donc modifiée.

Mots-clés : CDG Ik, ALG1, mannosyltransferase.

EP333. A NEW INBORN ERROR OF GLYCOSYLATION DUE TO DPM2 DEFICIENCY

W. Vleugels (1, 2), V. Race (1), R. Bammens (1), L. Keldermans (1), R. Barone (3, 4), F. Foulquier (2), G. Matthijs (1)

(1) Center for Human Genetics, Leuven, Belgium ; (2) Unité de Glycobiologie Structurale et Fonctionnelle UMR/CNRS 8576, IFR147, Lille, France ; (3) Department of Neuroscience - University of Catania, Catania, Italy ; (4) Centre for Inherited Metabolic Diseases - Department of Pediatrics - University of Catania, Catania, Italy

Contact : valerie.race@uz.kuleuven.ac.be

Congenital disorders of glycosylation (CDG) are a group of complex metabolic diseases. Since its first clinical description in 1980, about 40 different types have been identified. Most CDG are due to defects in the N-glycosylation pathway, the other ones affect the O-glycosylation pathway and a few types present with a combined $\mathrm{N}$ - and $\mathrm{O}$-glycosylation deficiency. We report here a patient with severe developmental delay, epilepsy and dysmorphic features, with a defect in the early steps on the $\mathrm{N}$-glycosylation process according to the transferrin isoelectric focusing (type I). Analysis of the lipid-linked oligosaccharides (LLO) showed an accumulation of the dol-PPGlcNAc2-Man5 structure. This pattern is compatible with a defect in the DPM1, ALG3 or MPDU1 genes, in which mutations have previously been described; however, no mutations were detected in those genes in this patient. We decided to further analyze DPM2 and SAC1 genes. The patient was found to be compound heterozygote for 2 mutations in the DPM2 gene: a splice mutation $(c .4-1 \mathrm{G}>\mathrm{C}$ ) and a point mutation (c.68A>G, p.Y23C). Hence, this patient presents with a novel type of CDG. The human dolichol-phosphatemannose (DPM) synthase is a heterotrimeric complex composed of DPM1,
DPM2 and DPM3. Until now, only mutations in DPM1 (the catalytic subunit) and more recently in DPM3 have been described. DPM2 is a hydrophobic protein of 84 amino acids, whose function is still not clear. It is reported in the literature that DPM2 could be involved in the regulation of the DPM synthase complex but also in the regulation of the glycosylphosphatidylinositols- $\mathrm{N}$-acetylglucosaminyltransferase. Further investigation of the effect of the DPM2 mutations by complementation analysis using Lec15 null hamster DPM2-deficient cells is ongoing.

Mots-clés : glycosylation, dolichol cycle, dysmorphies.

EP334. ÉVALUATION PILOTE DU DIAGNOSTIC MOLÉCU-
LAIRE DE LA MUCOVISCIDOSE VIA LE KIT XTAG ${ }^{\circ}$ CYSTIC FIBROSIS 71 KIT V2 DE LUMINEX

X. Pepermans (1), T. Leal (1), M. Philippe (1)

Centre de Génétique Humaine, Cliniques Universitaires, Saint-Luc, Bruxelles, Belgique

Contact : Xavier.Pepermars@uclouvain.be

Introduction La mucoviscidose (MIM219700) est la plus courante des maladies récessives graves dans la population caucasienne. Elle est causée par des mutations du gène CFTR (Cystic Fibrosis Transmembrane conductance Regulator), localisé en $7 \mathrm{q} 31.2$. Ce gène produit une protéine de 1480 acides aminés exprimée dans plusieurs tissus et glandes exocrines. L'expression phénotypique de la maladie est complexe et variable. Ses manifestations cliniques sont multiples et incluent des infections récurrentes sino-pulmonaires, une insuffisance pancréatique exocrine, de l'infertilité masculine, ainsi qu'une teneur élevée d'électrolytes dans la sueur. À ce jour, plus de 1500 différentes mutations ont été décrites (http ://www.genet.sickkids.on.ca/). Objectif L'objectif de cette étude est de comparer l'efficacité du diagnostic moléculaire de la mucoviscidose en dépistage en routine. Ainsi, afin de détecter les mutations et polymorphismes du CFTR, nous avons eu recours à deux méthodes différentes. La méthode xTAG ${ }^{*}$ Cystic Fibrosis 71 Kit v2 (Luminex, Canada) a été comparée à celle de INNO-LiPA CFTR 17+Tn Update combinée à INNOLiPA CFTR19 kit (Innogenetics, Belgium), utilisée dans cette étude comme méthode de référence. La méthode Luminex détecte 71 mutations délétères et 6 polymorphismes tandis que la méthode INNO-LiPA détecte 36 mutations et 3 polymorphismes. Méthodes Lors d'une première étape de validation, 30 échantillons d'ADN positifs par la méthode INNO-LiPA ont été analysés par le kit Luminex. Lors d'une deuxième étape, nous avons examiné des échantillons d'ADN provenant de différentes matrices biologiques ( 10 de chaque type) : sang total sur EDTA ; villosités choriales ou liquide amniotique, et sang total sur carte de Guthrie. L'ADN du sang total a été extrait par la méthode saline, ceux provenant de prélèvements fœtaux et de cartes de Guthrie ont été extraits par phenol-chloroforme. Chaque échantillon a été analysé en triplicat par 3 opérateurs différents à 3 jours différents. Finalement, 55 échantillons sans anomalie ont été comparés par les deux méthodes. Si une substitution a été détectée avec la méthode Luminex, mais pas avec la méthode INNO-LiPA, le séquençage exonique par BDT3.1/3130xl (Appliedbiosystems) a été réalisé pour confirmation. Résultats Dans toutes les étapes de validation et pour tous les échantillons d'ADN testés, les mêmes résultats pour chaque mutation ont été obtenus par les deux méthodes. La reproductibilité de la nouvelle méthode est identique à celle de la méthode de référence. Aucune différence significative inter-opérateur n'a été observée. Les 3 polymorphismes identifiés par la méthode Luminex (F508C, 1507V et I506) ont été confirmés par séquençage exonique du CFTR. Conclusions Le kit xTAG Cystic Fibrosis 71 Kit v2 de Luminex est robuste et permet la détection d'un plus large nombre de mutations CFTR. Cette technologie peut être appliquée comme méthode de dépistage en routine au laboratoire.

Mots-clés : mucoviscidose, Luminex, dépistage.

G335. INCLUSION D'UN EXON CRYPTIQUE DU GÈNE CFTR DANS UNE FORME ATYPIQUE DE MUCOVISCIDOSE

C. Costa (1, 2), V. Prulière Escabasse (3), L. Bassinet (4), L. Golmard (2), C. Gameiro (1), A. De Becdelièvre (1, 2), A. Aissat (2), M. Goossens (1, 2), E. Girodon-Boulandet $(1,2)$

(1) Laboratoire de Génétique, APHP CHU H Mondor, Creteil, France ; (2) Génétique INSERM U955 équipe 11, Université paris XII, Creteil, France ; (3) ORL, APHP CHU H Mondor, Creteil, France ; (4) Pneumologie-CRCM CHIC Créteil, France

Contact : catherine.costa@hmn.aphp.fr

La mucoviscidose est la plus fréquente des maladies autosomiques récessives chez les caucasiens. Plus de 1600 mutations du gène CFTR sont actuellement décrites comme responsables de formes classiques de mucoviscidose ainsi que de formes atypiques. La plupart des mutations sont 
situées dans les régions codantes du gène, incluant les sites consensus d'épissage, tandis que de rares mutations au milieu d'introns sont décrites, telles $3849+10 \mathrm{kbC}>\mathrm{T}$ et $1811+1.6 \mathrm{kbA}>\mathrm{G}$. Cependant un certain nombre d'allèles restent encore non identifiés, rendant le conseil génétique difficile, particulièrement lorsque le diagnostic est incertain. Nous décrivons ici le cas d'une femme de 25 ans présentant comme seul signe une bronchectasie disséminée, sans atteinte pancréatique, et un test de la sueur limite. Seule la mutation F508del à l'état hétérozygote ayant été identifiée après l'étude des 27 exons du gène et la recherche de grands réarrangements, nous avons réalisé une étude de l'ARNm extrait à partir des cellules épithéliales nasales. Un ARN de plus grande taille correspondant à une inclusion de $97 \mathrm{pb}$ entre les exons $6 \mathrm{~b}$ et $7 \mathrm{a}$ été mis en évidence. Cette inclusion correspond à une partie de la séquence de l'intron $6 \mathrm{~b}$, délétée de $4 \mathrm{pb}$ (GAAT), et qui est flanquée des séquences consensus d'epissage. Un codon stop apparaît au sein de cette séquence. L'analyse génomique de l'intron $6 \mathrm{~b}$ de la patiente montre la seule présence de cette délétion, c.1002-1112_1115delGAAT. Des études in silico (ESEfinder, Human Splicing Finder HSF) prédisent que ce motif (GAAT) pourrait être considéré comme un site silenceur d'epissage (ISS) et pourrait servir de matrice pour une hnRNP Al ribonucleoprotein. Cet élément cis intronique (GAAT) aiderait le spliceosome à ignorer les pseudo-exons et pourrait agir en tant que site de liaison pour des protéines dont le rôle serait de promouvoir l'exclusion d'exons. Ainsi la suppression de ce site aurait pour conséquence l'inclusion d'un pseudo-exon. Le taux de transcrit anormal est estimé à $20 \%$. Il persisterait donc un ARNm CFTR résiduel qui pourrait expliquer la forme modérée de mucoviscidose observée chez la patiente.

Mots-clés : mucoviscidose, ARN, pseudo-exon.

\section{-1P336. UTILISATION DE LA PCR EN TEMPS REEL POUR LA VALIDATION DE LA MLPA DANS LA RECHERCHE DE GRANDS RÉARRANGEMENTS GÉNIQUES}

O. Theisen (1), J. Leclerc (1), C.M. Dhaenens (1), E. Girodon (2), G. Lalau (1)

(1) Laboratoire de Toxicologie et Génopathies, Centre de Biologie Pathologie, CHRU de Lille, Lille, France ; (2) Service de Biochimie-Génétique, Hôpital Henri Mondor, Créteil, France

Contact : j-leclerc@chru-lille.fr

Les anomalies génétiques identifiées dans les maladies monogéniques sont principalement des mutations ponctuelles. Les grands réarrangements, délétions ou duplications pouvant toucher un exon, plusieurs exons ou le gène entier, représentent un pourcentage faible mais non négligeable des anomalies identifiées. Ils ne pouvaient initialement être suspectés que par la transmission uniparentale de marqueurs polymorphes intragéniques ou par l'absence d'amplification de produits de PCR. L'apparition ces dernières années de la technique MLPA (Multiplex Ligation-dependent Probe Amplification) a permis la recherche de ces réarrangements de façon simple et adaptée au diagnostic moléculaire au laboratoire. Quoique très fiable, cette technique peut cependant révéler de faux positifs. En effet, la présence d'un polymorphisme de l'ADN cible au niveau du site d'hybridation des sondes de MLPA, peut entraîner une absence d'amplification et la détection erronée d'une délétion. C'est pourquoi il peut être utile de disposer d'une technique permettant de confirmer les réarrangements identifiés en MLPA. Notre étude a donc consisté à valider l'utilisation de la quantification génique par PCR en temps réel en tant que technique de confirmation pour la recherche de réarrangements de deux gènes étudiés dans notre laboratoire, CFTR et NF2. Les grands réarrangements représentent 1 à $6 \%$ des mutations du gène CFTR et sont associés à des phénotypes sévères de mucoviscidose. Concernant le gène NF2, ils sont associés à des formes cliniques modérées de Neurofibromatose de type 2 et représentent près de $15 \%$ des mutations. Nous avons ainsi analysé par PCR en temps réel des patients pour lesquels une délétion, partielle ou complète, avait été préalablement identifiée par MLPA. Les résultats obtenus par MLPA ont tous été confirmés par PCR en temps réel, validant ainsi l'utilisation de cette technique comme méthode de confirmation de la MLPA. Par ailleurs, la PCR en temps réel apparaît être un bon outil de recherche en première intention de délétions plus fréquemment retrouvées dans certaines populations, comme la CFTRdele2-3 chez les patients slaves, ou de délétions pour lesquelles un déséquilibre de liaison avec une mutation ponctuelle a été décrit, comme la CFTRdele2-9 associée à la p.Leu997Phe. En conclusion, la PCR quantitative en temps réel semble être une méthode efficace, rapide et simple d'utilisation pour la validation des résultats obtenus par MLPA et pour la recherche ciblée de certaines délétions.
aP337. CONTRIBUTION À UNE MEILLEURE CONNAISSANCE DE LA PATHOGÉNICITÉ DE LA MUTATION I148T DU GÈNE CFTR RECHERCHEE LORS DU DÉPISTAGE NÉONATAL DE LA MUCOVISCIDOSE

M.P. Reboul (1), M.A. Delrue (1), C. Zordan (1), M.H. Dealbert (1), S. Labatut (1), B. Arveiler (1, 2), D. Lacombe (1, 2), F. Morice-Picard (1), A. Iron $(1,2)$

(1) Service de Génétique Médicale, Groupe Hospitalier Pellegrin, CHU de Bordeaux, France : (2) Laboratoire de Génétique Humaine, Université Victor Segalen-Bordeaux 2, France

Contact : marie-pierre.reboul@chu-bordeaux.fr

La stratégie de dépistage néonatal de la mucoviscidose choisie en France combine le dosage de la trypsine immunoréactive chez tous les nouveaux-nés avec une recherche de 30 mutations fréquentes chez une sélection d'entre eux, avant la pose éventuelle du diagnostic par dosage du chlorure sudoral. Cette procédure conduit inévitablement à l'identification - non recherchée - d'une part de porteurs sains hétérozygotes pour la maladie et d'autre part de nouveaux-nés porteurs de formes modérées (ou frontières) de la maladie, peu ou pas symptomatiques au moment du diagnostic et dont le pronostic est difficile à établir. Ces deux points constituent les difficultés essentielles du programme de dépistage néonatal et soulèvent la question du choix des mutations recherchées et d'une stratégie de dépistage sans biologie moléculaire. Il est donc maintenant nécessaire de réévaluer certaines mutations à rechercher lors de la première analyse de biologie moléculaire à J3. De plus cette réévaluation est importante pour le conseil génétique dans sa responsabilité quant à la prise en considération de ces mutations problématiques pour le diagnostic prénatal qui peut déboucher sur une demande d'interruption médicale de grossesse. Une des mutations actuellement recherchées lors du dépistage néonatal en France et pour lesquelles il est difficile d'établir précisément une relation génotype/phénotype est le faux sens I148T situé dans l'exon 4 du gène CFTR au niveau de la première boucle intracellulaire de la protéine CFTR. La mutation I148T a été décrite pour la première fois en 1994 en France comme mutation CF. Par la suite, d'autres observations ont été rapportées par différents auteurs, surtout dans des familles du sud de l'Italie chez lesquelles I148T semblait plutôt être associée à des formes cliniques sévères de la mucoviscidose, justifiant de ce fait sa présence dans le panel des mutations étudiées en $1^{\text {r }}$ intention dans les procédures de dépistage néonatal. Par la suite, les analyses exhaustives du gène CFTR chez la plupart des patients mucoviscidosiques porteurs de I148T ont montré son association en cis avec la mutation 3199del6 (ou 3195del6) de l'exon 17a, faisant beaucoup plus vraisemblablement de l'allèle complexe [I148T ; 3199del6] l'anomalie génique causale de mucoviscidose et remettant ainsi en question l'allèle I148T sans 3199 del6 comme mutation CF. Nous rapportons l'observation d'une femme de 36 ans porteuse du génotype [I148T]+[I148T;3199del6]. Son test sudoral est négatif et elle ne présente pas de symptomatologie pulmonaire. Cette observation peut contribuer à une meilleure connaissance de la pathogénicité de cette mutation et s'avérer utile dans le difficile débat sur le maintien ou non de certaines mutations dans le panel des mutations recherchées en première intention lors du dépistage néonatal de la mucoviscidose.

Mots-clés : mucoviscidose, dépistage néonatal, mutation $1148 \mathrm{~T}$.

aP338. ÉTUDE DES GRANDS RÉARRANGEMENTS DANS LE GENE CFTR PAR LA TECHNIQUE SQF-PCR

S. Hadj Fredj (1), M. Desgeorges (2), M. Boudeya (1), A. Bibi (1), R. Belhadj (1), M. Claustres (2), S. Fattoum (1), T. Messaoud (1)

(1) Laboratoire de biochimie, Hôpital d'enfants de Tunis, Tunisie ; (2) Laboratoire de génétique moléculaire, Institut universitaire de recherche clinique de Montpellier, France: (3) Centre de transfusion sanguine de Brest, France

Contact : taieb.messaoud@rns.tn

Depuis l'identification du gène CFTR en 1989, plus de 1700 mutations ont été identifiées chez les patients mucoviscidosiques. Toutefois, le pourcentage des allèles non identifiés est assez important dans la plupart des populations étudiées. La présence des grands réarrangements génomiques dans ce gène pourrait expliquer ce pourcentage assez élevé puisqu'ils échappent à la plupart des techniques de détection des mutations ponctuelles. C'est pourquoi, nous nous sommes intéressés pour la première fois à la recherche des grands réarrangements génomiques chez des patients tunisiens atteints de mucoviscidose chez qui au moins une mutation mucoviscidosique reste non identifiée. Ce travail a été effectuée par la technique de PCR semi-quantitative fluorescente: SQF-PCR (Semi Quantitative Fluorescent PCR Assay). C'est est une méthode semi-quantitative basée sur une PCR multiplexe de fragments fluorescents. L'amorce sens de chaque couple est marquée par le fluorophore 6-FAM. Elle permet ainsi, 
de façon rapide, de mettre en évidence des réarrangements de grande taille. Cette étude nous a permis d'identifier deux grandes délétions : la première est une grande délétion complexe déjà décrite (del 3-10,14b-16), allant de l'exon 3 à l'exon 10 et de l'exon $14 \mathrm{~b}$ à l'exon 16 , identifiée à l'état hétérozygote en association avec la mutation G542X localisée dans l'exon 11. La seconde est une nouvelle grande délétion, décrite pour la première fois de part le monde (del 3), identifiée à l'état hétérozygote; elle touche l'exon 3 et une partie des introns 2 et 3 . La détermination des points de jonction est en cours afin de déterminer la taille approximative de cette délétion. Cette recherche montre bien l'implication des grands réarrangements dans la distribution des mutations mucoviscidosiques dans notre population. Elle permet ainsi de diminuer nettement le pourcentage des mutations non identifiées, d'affiner le conseil génétique et d'améliorer la spécificité du diagnostic prénatal de mucoviscidose. À l'issue de cette étude, la famille présentant la délétion complexe del 3-10,14b-16 a bénéficié d'un diagnostic prénatal.

Mots-clés : mucoviscidose, SQF-PCR, grands réarrangements,

aP339. ÉTUDE DES REMANIEMENTS GÉNOMIQUES AU LOCUS CFTR

S. Quéméner (1, 2, 3), J.M. Chen (1, 3, 4), N. Chuzhanova (5), C. Bénech $(1,4)$, P. Stenson (6), K. Giteau (1, 2), M.P. Audrézet (1, 2), D.N. Cooper (6), C. Férec $(1,2,3,4)$

(1) Institut National de la Santé et de la Recherche Médicale (INSERM), Brest, France ; (2) Centre Hospitalier Universitaire (CHU) Morvan, Brest, France; (3) Université de Bretagne Occidentale (UBO), Brest, France; (4) Établissement Français du Sang (EFS) Bretagne, Brest, France ; (5) University of Central Lancashire, Preston, United Kingdom; (6) Institute of Medical Genetics, Cardiff, United Kingdom

Contact : sylvia.quemener@chu-brest.fr

Depuis la découverte du gène CFTR il y a 20 ans, plus de 1600 mutations pathologiques sont répertoriées. Cependant, aujourd'hui, très peu d'anomalies du nombre de copie (CNV, Copy Number variation) du gène CFTR ont été mises en évidence. Une vingtaine de délétions a été rapportée grâce à des techniques semi quantitatives de PCR, représentant 1 à $3 \%$ des anomalies causales de la mucoviscidose. Il est toutefois difficile de détecter des gains de copies avec les techniques actuelles. Afin de rechercher de nouveaux microou macro-réarrangements du gène CFTR entier, nous avons créé une puce CGH (Comparative Genomic Hybridization) dédiée à l'analyse du gène CFTR pour rechercher d'éventuels CNVs. Cette technologie permet d'observer à la fois des délétions, des duplications, ainsi que des anomalies dans les régions introniques et 5', 3'du gène. En effet, notre puce Agilent est constituée de 15000 oligonucléotides couvrant les $189 \mathrm{~kb}$ du gène CFTR et s'étendant $2 \mathrm{Mb}$ en amont et $700 \mathrm{~kb}$ en aval du gène. Elle contient 10000 oligonucléotides dans notre zone d'intérêt avec une sonde tous les $95 \mathrm{pb}$ au locus CFTR et une sonde tous les $250 \mathrm{~kb}$ dans les régions 5' et 3' flanquantes. Quatre milles oligonucléotides ont aussi été désignés sur chaque autosome et sur les chromosomes sexuels afin de normaliser les résultats de puce. Nous avons sélectionné une population de 162 patients atteints de mucoviscidose, originaires de différents pays : France, Espagne, États-Unis, Australie, République Tchèque, Irlande, Belgique, Ukraine, Turquie, Algérie, Tunisie. Pour chacun d'entre eux, le génotype est incomplet ce qui signifie qu'un allèle (ou les 2) n'a pas de mutation identifiée. Ceci correspond à 223 chromosomes analysés par CGH dédiée. Les résultats ont d'une part, validé la technique de CGH sur 16 délétions déjà connues dans le laboratoire et, d'autre part, mis en évidence 2 délétions ainsi que 5 duplications non observées par les techniques traditionnelles de PCR. Dans cette population analysée, nous avons évalué le ratio délétion: duplication à 3:1. Ce résultat est en accord avec différentes études de CNVs dans la population générale. Cette technique de CGH dédiée est complémentaire aux techniques actuelles de criblage du gène CFTR grâce à l'analyse des grands réarrangements, des régions introniques ainsi que 5' et 3'. Elle permet une observation rapide et sensible des CNVs permettant une analyse fine des points des cassure des anomalies afin de charactériser plus facilement les points de jonction.

Mots-clés : CFTR, CGH-array.

IP340. ÉTUDE DU GÈNE CFTR CHEZ 27 PATIENTS ALGÉRIENS F. Cabet (1), K. Radoui (2), D. Boggio (3), G. Bellon (3), Y. Morel (1)

(1) Laboratoire d'Endocrinologie Moléculaire et Maladies Rares, Centre de Biologie et de Pathologie Est, CHU Lyon, Bron, France ; (2) Service de Pneumo-Allergologie, Canastel, CHU Oran, Algérie; (3) Service de Pneumologie, Hôpital-Mère Enfant, CHU Lyon, Bron, France Contact : faiza.cabet@chu-lyon.fr

La fréquence des mutations du gène CFTR n'est pas connue en Afrique du Nord et en particulier en Algérie. Très peu d'études ont été rapportées dans la littérature car pendant longtemps on a considéré la mucoviscidose comme une pathologie exceptionnelle dans cette région. Depuis le clonage du gène CFTR en 1989, plus de I 500 variations de séquence ont été répertoriées par le Consortium "Cystic Fibrosis genetic Analysis consortium ». Plusieurs études montrent, pour certaines mutations, de grandes variations de fréquence entre les populations d'origines ethniques différentes. Par exemple, la fréquence de la F508del varie selon un gradient Nord-Sud ( $100 \%$ au nord du Danemark et $24 \%$ en Turquie). Chez 27 enfants algériens non apparentés atteints de mucoviscidose, nous avons identifié 19 mutations différentes, certaines avec une fréquence significativement différente de celle habituellement décrite. Ainsi, la mutation $711+1 \mathrm{G}>\mathrm{T}$ rarement observée dans les autres populations (1.3\% en Italie du sud et $0,1 \%$ aux USA) est la mutation la plus fréquente ( $19 \%)$ après la F508del $(20 \%)$. Trois autres mutations ont une fréquence inhabituellement élevée par rapport à d'autres séries : $9,5 \%$ pour Q290X et $5,6 \%$ pour $1898+3 \mathrm{~A}>\mathrm{G}$ et $3272-26 \mathrm{~A}>\mathrm{G}$ (pour cette dernière, la fréquence n'est que de $0,3 \%$ en Europe). Les autres mutations identifiées sont: N1303K, G542X, $2183 \mathrm{AA}>\mathrm{G}, \mathrm{L} 227 \mathrm{R}, 3120+1 \mathrm{G}>\mathrm{A}, \mathrm{H} 199 \mathrm{R}, \mathrm{E} 1104 \mathrm{X}, \mathrm{S} 549 \mathrm{R}, \mathrm{G} 551 \mathrm{D}$, 2789+5G>A, A455E, S997F, K1165X, 4472delT. Cette étude confirme le spectre mutationnel spécifique de la mucoviscidose en Algérie avec néanmoins des fréquences différentes de celles publiées par Loumi en 2008. Ces résultats démontrent l'intérêt d'inclure ces mutations parmi les plus fréquentes à rechercher chez les conjoints d'hétérozygotes dans ces populations en vue d'un conseil génétique approprié.

Mots-clés : CFTR, mutations, Algérie

IP341. IMPLICATION DU GÈNE CFTR DANS LA SURVENUE DE L'AGÉNÉSIE BILATÉRALE DES CANAUX DÉFÉRENTS (ABCD) S. Hadj Fredj, M. Boudaya, S. Oueslati, A. Bibi, R. Belhadj, T. Messaoud Laboratoire de Biochimie, Hôpital d'enfants de Tunis, Tunisie

Contact : taieb.messaoud@rns.tn

L'agénésie bilatérale des canaux déférents (ABCD) est une affection congénitale représentant 1 à $2 \%$ des causes d'infertilité masculine et 9 à $25 \%$ des azoospermies obstructives. Récemment, différentes études moléculaires ont permis de montrer que des mutations mucoviscidosiques, pouvaient rendre compte des bases génétiques de cette forme de stérilité et que certains polymorphismes localisés dans le gène CFTR pouvaient fortement être associés à l'agénésie bilatérale des canaux déférents. Le nombre important de stérilité masculine par agénésie bilatérale des canaux déférents observés dans notre pays nous a poussé à orienter notre étude sur la recherche des mutations et des polymorphismes mucoviscidosiques (TGmTn, V201M et M470V) impliqués dans cette affection dans notre population. À cet effet, nous avons étudié une population de sexe masculin dont l'age varie entre 33 ans et 44 ans chez qui le diagnostic d'agénésie bilatérale des canaux déférents a été confirmé. Cette étude a été menée par la combinaison de différentes techniques de biologie moléculaire telles que la chromatographie liquide à haute performance en milieu dénaturant (DHPLC), l'électrophorèse sur gel en gradient dénaturant (DGGE) et le séquençage automatique. Notre travail a porté tout d'abord sur la recherche des mutations du gène CFTR chez ces patients agénésiques en explorant 9 exons du gène CFTR. Cette étude nous a permis d'identifier 5 différentes mutations : R74W, F508del, G542X, W1282X et D1270N. Le second volet de notre travail a porté sur l'étude de trois polymorphismes: TGmTn, $\mathrm{V} 201 \mathrm{M}$ et M470V situés respectivement dans l'intron 8 et dans les exons $6 \mathrm{a}$ et 10 . Le variant $5 \mathrm{~T}$ a été identifié chez trois de nos malades agénésiques ; pour les trois cas observés, le variant $5 \mathrm{~T}$ est associé avec la répétition (TG) 12 ce qui suggère son application au niveau de la modulation de l'épissage de l'exon 9. À l'issue de la recherche des polymorphismes $\mathrm{V} 201 \mathrm{M}$ et M470V, nous avons pu identifié 2 malades portant le variant V201M à l'état hétérozygote et 7 malades portant le polymorphisme M470V. Dans la présente étude, on a pu montré d'une part que les variants $5 \mathrm{~T}, \mathrm{~V} 201 \mathrm{M}$ et M470V sont plus fréquents chez les hommes atteints d'une agénésie bilatérale des canaux déférents par rapport à la population générale et que des mutations responsables de mucoviscidose sont identifiées chez ces patients agénésiques ce qui permet sans ambiguïté de confirmer l'implication des mutations et des polymorphismes mucoviscidossiques dans la survenue de cette forme de stérilité masculine. L'étude moléculaire du gène CFTR sert à connaitre et comprendre les bases génétiques de cette pathologie et à imposer un conseil génétique jugé nécessaire pour les couples qui veulent avoir recours à une procréation médicalement assistée par injection intracytoplasmique des spermatozoïdes pour éviter tout risque d'agénésie bilatérale des canaux déférents ou encore tout risque de mucoviscidose jusqu'au jour où tous les facteurs determinants de cette pathologie seront totalement élucidés.

Mots-clés : mucoviscidose, mutations, $\mathrm{ABCD}$. 
IP342. MISE EN ÉVIDENCE DE MUTATIONS DU GÈNE CFTR CHEZ DES PATIENTS CONSULTANT POUR HYPOFERTILITÉ AVEC CANAUX DÉFÉRENTS PRÉSENTS À L'EXAMEN CLINIQUE

O. Theisen (1), J. Leclerc (1), J.M. Rigot (2), F. Marcelli (2), V. Mitchell (3), J.J. Lafitte (4), A. Moerman (5), G. Lalau (1)

(1) Laboratoire de Toxicologie et Génopathies, Centre de Biologie Pathologie, CHRU de Lille, Lille, France; (2) Service d'Andrologie, Hôpital Calmette, CHRU de Lille, Lille, France : (3) Laboratoire de Spermiologie, Hôpital Calmette, CHRU de Lille, Lille, France; (4) Service de Pneumologie et Oncologie Thoracique, Hôpital Calmette, CHRU de Lille, Lille, France; (5) Service de Génétique Clinique, Hôpital Jeanne-de-Flandre, CHRU de Lille, Lille, France

Contact : j-leclerc@chru-lille.fr

L'agénésie bilatérale des canaux déférents $(\mathrm{ABCD})$ est responsable d'une azoospermie dite obstructive et représente 1 à $3 \%$ des causes de stérilité masculine. Cette pathologie fait partie des affections associées aux variations du gène CFTR. Selon la littérature, 78 à $92 \%$ des sujets atteints d'ABCD sont porteurs d'au moins une mutation du gène CFTR. 127 patients consultant pour hypofertilité dans notre Centre présentaient une hypovolémie spermatique, une azoo- ou une oligospermie et un taux de fructose séminal abaissé. Une altération du gène CFTR était identifiée sur $85 \%$ des allèles étudiés : $92 \%$ des patients présentaient au moins une mutation. Parmi les mutations identifiées, $40 \%$ sont des mutations « CFcausing » (p.F508del : $33 \%$ ) et $60 \%$ des variations pouvant être considérées «CFTR-related disorders associated " (allèle IVS8-T5 : $23 \%$ ) ou « unproven or uncertain clinical relevance». Parmi les hétérozygotes composites ou supposés tels en l'absence d'étude familiale. 1 \% est porteur de 2 mutations sévères (homozygote p.F508del), $69 \%$ sont porteurs d'1 mutation sévère et d' 1 mutation modérée, $30 \%$ sont porteurs de 2 mutations modérées ou d'effet délétère incertain. L'examen clinique retrouvait une absence ou une anomalie des canaux déférents à la palpation chez 113 d'entre eux. Chez 14 patients, les deux canaux déférents étaient accessibles à la palpation ; ils présentaient un spermogramme évocateur (hypovolémie, azoospermie (10 patients) ou oligospermie (4 patients), fructose séminal abaissé) ; 9 étaient porteurs de 2 variations du gène CFTR et les 5 autres d'1 seule. L'examen clinique ne constatait aucune anomalie génitale chez 10 d'entre eux $(71 \%)$ et mettait en évidence une dilatation des épididymes chez les 4 autres $(29 \%)$ : l'échographie génito-urinaire était strictement normale pour 11 sujets ( $79 \%$ ) et montrait une absence des vésicules séminales dans 1 cas. 10 patients avaient bénéficié d'un test de la sueur, pathologique chez 6 d'entre eux $(60 \%)$, des valeurs de chlorures normales étaient constatées chez les 3 patients oligospermiques testés. 2 patients qui ne présentaient pas d'anomalie clinique et échographique et avaient un spermogramme moins altéré (oligospermie et fructose légèrement diminué) étaient hétérozygotes composites pour 2 variations d'effet délétère au plus très modéré. Des altérations du gène CFTR sont rencontrées chez des patients dont les déférents sont présents mais qui présentent un spermogramme évocateur ; d'autre part, le fait que certains patients aient pu procréer de façon naturelle quelques années auparavant laisse suggérer une aggravation progressive, comme pour les autres atteintes de la mucoviscidose, de l'atteinte génitale au cours du temps.

Mots-clés: infertilité masculine, gène CFTR, agénésie des canaux déférents.

\section{IP343. IDENTIFICATION D'ANOMALIES STRUCTURALES DES CELLULES CILIÉES DE L'ÉPITHELIUM NASAL SANS TRA- DUCTION CLINIQUE CHEZ LES PATIENTS ATTEINTS D'AMAUROSE CONGÉNITALE DE LEBER PORTEURS DE MUTATIONS DU GÈNE CEP290}

J.M. Rozet (1), J. Kaplan (1), J.F. Papon (2), A. Coste (2), B. Louis (3), E. Escudier (3), I. Perrault (1)

(1) INSERM U781 \& Université Paris-Descartes, CHU Necker, Paris, France; (2) Service ORL, CHU Henri-Mondor, Créteil, France; (3) INSERM U 841\& Université Pierre-et-Marie-Curie, CHU Henri Mondor, Créteil, France

Contact : jean-michel.rozet@inserm.fr

Contrairement aux altérations du gène CEP290 à l'origine des syndromes de Joubert et de Senior-Loken, celles qui ont été identifiées chez les patients atteints d'amaurose congénitale de Leber (ACL) semblent n'avoir aucune traduction pathologique en dehors de la rétine. Toutefois, la localisation de CEP290 au niveau du cil connecteur des photorécepteurs, son interaction avec RPGR et plus généralement son rôle dans la fonction ciliaire, nous a incités à rechercher d'autres manifestations cliniques chez les patients atteints d'ACL. Cela a déjà été illustré pour les altérations du gène la fonction des cellules de l'oreille interne, des muqueuses respiratoires, des spermatozoïdes et certainement d'autres cellules ainsi qu'en témoignent les cas d'hypoacousie, d'infections répétées des voies respiratoires, de stérilité et même de dyskinésie ciliaires rapportés chez les patients porteurs de mutations de ce gène. Sept patients (âges compris entre 8 et 40 ans ; age moyen 17,5 ans) appartenant à 6 familles ont accepté la réalisation d'un audiogramme, d'un test d'olfaction et d'un examen endoscopique de la muqueuse nasale avec prélèvement de cellules en vue de l'étude de la cinétique du battement ciliaire par vidéomicroscopie à haute vitesse et de celle de l'ultrastructure ciliaire. Parmi les sept patients, 1/7 est homozygote pour la mutation intronique c. $2991+1655 \mathrm{~A}>\mathrm{G}, 1 / 7$ est hétérozygote composite pour cette mutation et une mutation non sens et les 5 autres sont homozygotes ou hétérozygotes composites pour des mutations nonsens ou des mutations touchant les sites consensus d'épissage. L'interrogatoire sur d'éventuels antécédents ORL a montré que $6 / 7$ de ces patients souffraient en permanence d'une congestion muqueuse nasale, parfois associée à une obstruction nasale chronique (3/6), 4/7 avaient des antécédents d'otites à répétition, $3 / 7$ des antécédents de bronchites répétées, $2 / 7$ de rhinopharyngites et de sinusites, $2 / 7$ avaient une hypoaccousie discrète de transmission et enfin 1/7 (40 ans) était stérile par azoo-asthénospermie. Quelque soit leur génotype, les patients se sont révélés porteurs d'anomalies ciliaires. Chez les 7 patients les cils étaient courts et rigides; chez $3 / 7$ patients le nombre de cellules battantes était significativement diminué ; chez $2 / 7$ patients, incluant l'homozygote pour la mutation intronique, la fréquence du battement ciliaire était très diminuée ; chez tous les patients, il a été noté des anomalies fréquentes de l'ultrastructure ciliaire : absence de microtubules centraux, absence de bras de dyneine et même déciliation chez un patient. Ces constatations démontrent qu'en dépit de l'absence de pathologies extraoculaires, les mutations du gène CEP290, y compris la mutation intronique, s'expriment au-delà de la rétine, apportant un argument à l'entrée de quelques sous-types génétiques d'amaurose de Leber dans la grande famille des ciliopathies.

Mots-clés: amaurose congénitale de Leber, mutations CEP290, ciliopathie.

"P344. INTÉRÊT DE L'ÉTUDE MOLÉCULAIRE DES GÈNES BBS DEVANT LA DÉCOUVERTE D'UN HYDROMÉTROCOLPOS ASSOCIÊ À UNE POLYDACTYLIE EN ANTÉNATAL ÉVOQUANT UN SYNDROME DE MCKUSICK-KAUFMAN - BARDET-BIEDL

E. Schaefer (1), M. Durand (1), C. Stoetzel (2), B. Doray (1), B. Viville (3), S. Hellé (2), J.M. Danse (2), C. Hamel (4), P. Bitoun (5), A. Goldenberg (6), S. Finck (7), L. Faivre (8), S. Sigaudy (9), M. Holder (10), M.C. Vincent (11), V. Marion (2), D. Bonneau (12), A. Verloes (13), I. Nisand (3), J.L. Mandel (14), H. Dollfus (1, 2)

(1) Service de Génétique Médicale: centre de référence anomalies du développement Grand-Est et centre de référence affections génétiques ophtalmologiques (CARGO), Hôpitaux Universitaires de Strasbourg, Strasbourg, France; (2) Laboratoire de Génétique Médicale EA 3949, Équipe Avenir-Inserm, Faculté de Médecine de Strasbourg, Université Louis-Pasteur, Strasbourg, France ; (3) Service de Gynécologie Obstétrique, Hôpitaux Universitaires de Strasbourg, Strasbourg, France; (4) Service d'ophtalmologie, CHU Hôpital Gui-de-Chauliac, Montpellier, France; (5) Consultation de génétique ophtalmologique, Hôpital Jean-Verdier, Bondy, France: (6) Unité de génétique clinique, CHU Hôpital Charles-Nicolle, Rouen, France; (7) Service de pédiatrie, CHR, Haguenau, France; (8) Centre de génétique médicale, CHU Hôpital d'Enfants, Dijon, France; (9) Département de génétique médicale - Unité de génétique clinique, Hôpital des enfants de la Timone, Marseille, France; (10) Service de génétique clinique Guy Fontaine, CHRU de Lille - Hôpital Jeanne-deFlandre, Lille, France; (11) Laboratoire de diagnostic génétique, CHU, Strasbourg, France; (12) Service de génétique, CHU, Angers, France; (13) Département de génétique, APHP-CHU Robert-Debré, Paris, France: (14) Collège de France, Paris, France

Contact : helene.dollfus@chru-strasbourg.fr

L'hydrométrocolpos est une malformation rare définie par l'accumulation de secrétions dans le vagin ou l'utérus secondaire à une obstruction vaginale congénitale (atrésie ou imperforation) et responsable d'une masse kystique pelvienne. Selon son expression clinique, le diagnostic peut être plus ou moins précoce. Dans la période anténatale, il se manifeste par une masse liquidienne abdominale de taille variable qui peut engendrer, dans les formes extrêmes, à la naissance, une insuffisance rénale terminale d'origine mécanique ou une insuffisance respiratoire par hypoplasie pulmonaire et entrainer le décès. La découverte d'un hydrométrocolpos en prénatal doit faire rechercher des signes associés évoquant un syndrome malformatif à type de malformations génitales ou extra-génitales. La malformation la plus fréquemment retrouvée est la polydactylie, qui doit alors faire 
suspecter 2 principaux syndromes : le syndrome de McKusick-Kaufamn (MKKS) et le syndrome de Bardet-Biedl (BBS). Dans ces deux syndromes, I'hydrométrocolpos et la polydactylie peuvent être les seuls signes présents à l'échographie et c'est le bilan post-natal à la recherche d'autres malformations ou l'évolution clinique qui permettra de les distinguer. En effet, la présence de malformations cardiaques ou gastrointestinales est plus fréquente dans MKKS et les anomalies rénales primitives se retrouvent presque exclusivement dans BBS. D'autre part, les patients atteints du BBS peuvent développer dans l'enfance une obésité, une rétinopathie pigmentaire, un retard mental et une insuffisance rénale progressive. Le pronostic de ceux syndromes étant différents, il est important de pouvoir poser un diagnostic et précoce pour le conseil génétique et la prise en charge de ces patients. À ce jour, 14 gènes sont identifiés dans le syndrome de BardetBiedl (BBS1 à BBS14) alors qu'un seul gène (MKKS-BBS6) a été décrit comme impliqué dans le syndrome de McKusick-Kauffman. Au vue de la difficulté à distinguer ces deux syndromes en période néonatale, nous avons repris rétrospectivement la cohorte de 350 familles BBS de notre laboratoire et nous avons identifié 7 patients pour lesquels le diagnostic de MKKS avait été éyoqué à la naissance et chez qui des mutations dans divers gènes BBS (BBS2, BBS6, BBS8, BBS10 et BBS12) ont été trouvées, posant le diagnostic final de BBS. Nous développerons l'histoire familiale, le phénotype et le génotype de ces patients. Enfin, à partir de cette étude, nous montrerons l'importance de l'étude moléculaire des gènes BBS en cas d'hydrométrocolpos associé à une polydactylie en période anté ou néonatale pour le diagnostic, le pronostic et le conseil génétique.

Mots-clés : syndrome de McKusick-Kaufman, syndrome de Bardet-Biedl, diagnostic moléculaire.

EP345. BASES MOLÉCULAIRES D'UNE FORME SYNDROMIQUE DE CILIOPATHIE ASSOCIANT DYSKINÉSIE CILIAIRE PRIMITIVE ET SYNDROME DE USHER

M. Legendre (1, 2), P. Duquesnoy (2), H. Tenreiro (1), L. Vincensini (2), A. Deschildre (3), A. Delattre (4), D. Escalier (2), E. Escudier (1, 2), S. Amselem $(1,2)$

(1) Service de Génétique et d'Embryologie médicales, APHP, Hôpital Trousseau, Paris, France; (2) Inserm U933, Hôpital Trousseau, Paris, France ; (3) Service de Pneumologie pédiatrique, Hôpital Jeanne-de-Flandres, Lille, France; (4) Service d'ORL, Hôpital Roger-Salengro, Lille, France

\section{Contact : serge.amselem@trs.aphp.fr}

Les cils sont présents à la surface de nombreuses cellules. Ces organelles, de structure apparentée au flagelle du spermatozoïde, peuvent être classés selon l'organisation des microtubules de leur cytosquelette appelé axonème : cils mobiles comme ceux des cellules épithéliales respiratoires, cils primaires comme ceux des cellules nodales embryonnaires, et structures apparentées comme le cil connecteur des photorécepteurs et le kinocilium des cellules de l'oreille interne. En pathologie moléculaire, des mutations de plusieurs gènes ont été identifiées à l'origine de ciliopathies. Ainsi, 10 gènes sont impliqués dans les dyskinésies ciliaires primitives (DCP) (prévalence 1/15000), maladie respiratoire résultant d'un défaut de mobilisation du mucus par dysfonctionnement des cils respiratoires, associée à une infertilité masculine et dans environ $50 \%$ des cas à un situs inversus. Plus rarement, les DCP sont syndromiques, associées notamment à une rétinite pigmentaire ; cette forme particulière s'explique par des anomalies de RPGR impliqué dans le transport de protéines le long des axonèmes des cils et des photorécepteurs. Par ailleurs, dans les syndromes de Usher (prévalence 1/30000) caractérisés par une atteinte sensorielle (rétinite pigmentaire et surdité de perception) sans atteinte respiratoire, plusieurs gènes ont également été identifiés. Nous rapportons ici les bases moléculaires d'un syndrome complexe associant, chez deux frères consanguins, une DCP avec absence des bras de dynéine externes et un syndrome de Usher de type I, faisant suspecter un défaut génétique touchant une protéine commune à différents types d'axonèmes. L'analyse moléculaire (ADN et ARN) a mis en évidence une délétion homozygote des exons 3 et 4 de DNAI2 (p.Ala62GlyfsX115), qui résulte très probablement d'une recombinaison illégitime entre deux séquences Alu situées dans les introns 2 et 4. DNAI2 étant connu pour son implication dans les DCP non syndromiques, ces données nous ont incités à rechercher chez ces 2 frères un second gène pouvant rendre compte du syndrome de Usher. L'analyse de USHIG, localisé à $650 \mathrm{~kb}$ de DNAI2 au sein d'une région commune d'homozygotie, a mis évidence une mutation non sens homozygote (p.Lys218X), qui génèrerait une protéine tronquée du domaine SAM d'interaction avec l'harmonine. Les anomalies moléculaires des deux gènes, non décrites à ce jour, ont été retrouvées à l'état hétérozygote chez les parents asymptomatiques des cas index. Alors que l'expression syndromique de plusieurs ciliopathies s'explique aisément par les fonctions pléiotropes de certaines protéines ciliaires, cette observation illustre la survenue de deux ciliopathies rares secondaires à des mutations homozygotes de deux gènes distincts étroitement liés.

Mots-clés : dyskinésie ciliaire primitive, syndrome de Usher, DNAI2, USHIG.

IP346. IDENTIFICATION DE 39 NOUVELLES MUTATIONS DANS LE SYNDROME DE BARDET-BIEDL : LA DIFFICULTÉ DES MUTATIONS PRIVÉES DANS LE DIAGNOSTIC D'UNE MALADIE HÉTÉROGÊNE

J. Muller (1, 2), C. Stoetzel (3), M.C. Vincent (1), V. Laurier (3), J.M. Danse (3), S. Hellé (3), V. Marion (3), V. Bennouna-Greene (3), S. Vicaire (2), A. Megarbane (4), J. Kaplan (5), V. Drouin-Garraud (6), M. Hamdani (7), S. Sigaudy (8), C. Francannet (9), J. Roume (10), P. Bitoun (11), A. Goldenberg (6), N. Phillip (12), S. Odent (13), J. Green (14), M. Cossée (1), E.E. Davis (15), N. Katsanis (15), D. Bonneau (16), A. Verloes (17), J.L. Mandel (1, 2, 18), H. Dollfus (3)

(1) Laboratoire de Diagnostic Génétique, CHU Strasbourg Nouvel Hôpital Civil, Strasbourg, France : (2) IGBMC (Institut de Génétique et de Biologie Moléculaire et Cellulaire), CNRSIINSERM/Université de Strasbourg, IIIkirch, France; (3) Laboratoire de Génétique Médicale EA3949, Equipe AVENIR-Inserm, Université de Strasbourg, Faculté de Médecine, Strasbourg, France; (4) Unité de Génétique Médicale, Université Saint Joseph Faculté de Médecine, Beirut, Liban; (5) Laboratoire de Génétique Médicale, Hôpital Necker-Enfants Malades, Paris, France; (6) Unité de Génétique Clinique, Hôpital Charles Nicolle, Rouen, France ; (7) Hôpital 20 Août 1953, Casablanca, Maroc; (8) Service de Génétique Médicale, CHU de la Timone, Marseille, France ; (9) Service de Génétique, Centre Hospitalier Universitaire de Clermont-Ferrand., France: (10) Service de Génétique Médicale, CHI Poissy-St-Germain, Poissy, France ; (1I) Service de pédiatrie, Hôpital Jean-Verdier, Bondy, France; (12) Hôpital de la Timone enfants, Service de Pédiatrie et de Génétique Médicale, Marseille, France; (13) Unité de Génétique Médicale, CHU Hôpital Sud, Rennes, France; (14) Department of Genetics, Memorial University of Newfoundland St John's, Canada ; (15) McKusick-Nathans Institute of Genetic Medicine, Johns Hopkins University School of Medicine, Baltimore, Maryland, États-Unis ; (16) Service de Génétique, CHU, Angers, France; (17) Unité de Génétique Clinique, Hôpital Robert Debré, Paris, France; (18) Chaire de Génétique Humaine, Collège de France, Illkirch, France

Contact : jean.muller@igbmc.fr

Le syndrome de Bardet-Biedl (SBB) (OMIM 209900) est une maladie autosomique récessive de la classe des ciliopathies, définie par un ensemble de signes cliniques incluant: rétinopathie pigmentaire précoce, obésité, polydactylie, hypogonadisme, déficit cognitif variable et anomalies rénales. BBS est caractérisé par une hétérogénéité génique importante avec 14 gènes identifiés à ce jour. Ces gènes sont retrouvés mutés dans environ $75 \%$ des familles atteintes. Les gènes BBS1 et BBS10 sont les plus souvent impliqués chez les patients atteints : $20 \%$ de fréquence respective pour chacun de ces 2 gènes, $8 \%$ pour BBS 12 , moins de $5 \%$ pour les autres gènes BBS (BBS11, BBS13 et BBS14 ont été identifiés dans 1 seule famille).

Dans cette étude, nous avons analysé les 12 premiers gènes BBS dans une cohorte de 185 familles de patients en combinant des stratégies d'analyse variées: techniques de pré-screening (SSCP, dHPLC...), de séquençage direct et en particulier pour les familles consanguines des puces SNP pour la détection de régions chromosomiques candidates par cartographie homozygote. Nous avons ainsi pu identifier au moins une mutation dans 146 familles $(78,9 \%)$. Les 39 familles sans mutation $(21,1 \%)$ font l'objet d'études plus approfondies incluant la recherche de mutations par délétion, la recherche de mutations dans les promoteurs ou encore l'identification de nouveaux gènes BBS. Au total 96 mutations ont pu être recensées dans 11 gènes BBS, dont 39 sont des nouvelles mutations jamais décrites à ce jour. Ce résultat confirme le niveau élevé de mutations privées dans le syndrome de Bardet-Biedl.

La forte hétérogénéité génétique (plus de 200 exons à séquencer), et la nature privée des mutations ponctuelles identifiées rendent difficile l'identification des anomalies moléculaires chez les patients atteints de BBS au sein d'un laboratoire de diagnostic génétique, qui ne dispose pas des outils haut débit adaptés à cette complexité. Cela retarde la prise en charge des familles sur le plan du conseil génétique. Il est à noter que nous n'avons pas pu détecter de mutation dans le gène BBS11. Ce gène a été rattaché au syndrome par l'identification d'une seule mutation faux sens dans une unique famille BBS. Par contre plusieurs autres mutations du gène BBS11 ont été associées à un phénotype très différent : la myopathie des ceintures autosomique récessive de type $2 \mathrm{H}$ (LGMD2H).

Mots-clés : syndrome de Bardet-Biedl, mutation, diagnostic. 
IP347. SYNDROME DE BARDET BIEDL: STRATÉGIE DIAGNOSTIQUE POUR LES FAMILLES CONSANGUINES

M.C. Vincent (1, 2), N. Dondaine (1), C. Stoetzel (2), C. Thibeault (3), F. Pleniak (3), M. Antin (1), A.S. Jaeger (1), S. Samimi(1), E. Scherrer (1), V. Kremer (1), J. Amiel (4), C. Antignac (4), B. Leheup (5), J. Roume (6), B. Isodor (7), F. Giulano (8), C. Vilain (9), M. Holder (10), H. Dollfus (2, 11), J.L. Mandel $(1,3)$

(1) Laboratoire de Diagnostic Génétique, Hôpitaux Universitaires de Strasbourg, France: (2) Laboratoire de Génétique Médicale, EA3949. Faculté de Médecine, Hôpitaux Universitaires de Strasbourg, France ; (3) IGBMC (CNRS/INSERM/ULP), Illkirch, France; (4) Département de Génétique, Groupe hospitalier universitaire Necker Enfants malades, Paris, France; (5) Service de Médecine Infantile et Génétique Clinique, Centre Hospitalier Universitaire Nancy Bradois, France; (6) Unité de Génétique Clinique, Service de Cytogénétique et Biologie de la Reproduction, CHI Poissy, Saint-Germain en Laye, France ; (7) Unité de Génétique Clinique, Centre hospitalier Universitaire de Nantes, France ; (8) Unité de Génétique Clinique, Centre Universitaire de Nice, France; (9) Service de Génétique Médicale, Université Libre de Bruxelles, Belgique; (10) Service de Génétique Médicale, Centre Hospitalier Régional Universitaire Lille, France; (11) Service de Génétique Médicale, Hôpitaux Universitaires de Strasbourg, France

Contact : marie-claire.vincent@chru-strasbourg.fr

Le syndrome de Bardet Biedl (BBS [MIM209900]) associe une rétinopathie pigmentaire, une obésité, une polydactylie, des malformations rénales et génitales et des troubles cognitifs. Son incidence est de $1 / 150000$ individus dans la population nord européenne, mais peut être beaucoup plus élevée dans les populations à forte consanguinité. Ce syndrome, majoritairement autosomique récessive, est génétiquement hétérogène : 14 gènes publiés à ce jour (BBS1_BBS14) représentant plus de 200 exons à étudier. Nous rapportons l'étude de 12 cas ( 6 sporadiques et 6 familiaux) de sujets appartenant à une famille consanguine BBS par puce de génotypage SNP (Affymétrix $10 \mathrm{~K}$ ou $50 \mathrm{~K}$ ). Cette étape préliminaire permet de cibler la recherche de mutations sur un ou un petit nombre de gènes BBS candidats pour un séquençage direct ou pour une étude par l'association pré-screening HRM et séquençage du variant détecté. Cette stratégie de réduction et ciblage du (des) gène(s) BBS à analyser a limité la recherche de mutations sur 1 à 4 gènes BBS maximum pour un patient donné. Nous avons obtenu un taux de détection de mutation positive de $75 \%$, équivalent à celui rapporté par les études exhaustives des 12 premiers gènes dans les familles BBS publiées. Cet outil diagnostic a permis pour 5 familles l'identification de mutations privées dans des gènes BBS de faible fréquence (BBS2, BBS5, BBS6 et BBS7) qui ne font pas partie des 3 gènes étudiés en première intention (BBS1, BBS10 et BBS12 de fréquence respective $25 \%, 20 \%$ et $10 \%$ ). Cette stratégie rapide, efficace et peu coûteuse permet d'offrir une optimisation du conseil génétique aux familles BBS consanguines.

Mots-clés : Bardet biedl, famille consanguine, puce SNP.

\section{MP348. DESCRIPTION D'UNE DISOMIE UNIPARENTALE DU CHROMOSOME 12 RESPONSABLE D'UNE MALADIE DE WIL- LEBRAND DE TYPE 3}

P. Boisseau (1), M. Fouassier (2), M. Giraud (1), O. Pichon (3), C. Thomas (1), P. Talarmain (1), C. Ternisien (2), M. Trossaert (2), S. Bezieau (1) (1) Laboratoire de Génétique Moléculaire, CHU Hôtel-Dieu, Nantes, France ; (2) Centre Régional de Traitement de l'Hémophilie, Laboratoire d'Hématologie, CHU Hôtel-Dieu, Nantes, France; (3) Laboratoire de Cytogénétique, CHU Hôtel-Dieu, Nantes, France

Contact : pierre.boisseau@chu-nantes.fr

La maladie de Willebrand (VWD) est une maladie hémorragique héréditaire de sévérité variable, due à des anomalies qualitatives ou quantitatives du facteur Willebrand (VWF). La VWD de type 3 est la forme la plus sévère de cette pathologie. Elle est caractérisée par un déficit quantitatif total en facteur Willebrand et sa présentation clinique se traduit par des manifestations hémorragiques graves. C'est une maladie autosomique récessive rare dont le gène responsable se situe en 12p13.3. Les parents de ces patients sont considérés comme transmetteurs mais ne présentent pas systématiquement les phénotypes clinique ou biologique de la maladie. Nous présentons ici une patiente de 5 ans adressée au Centre de Traitement des Hémophiles de Nantes pour un saignement gingival prolongé. Les résultats biologiques montraient un taux de FVIII :C à 1 \% (norm $>50 \%$ ) et pour le VWF: un dosage d'activité du cofacteur à la ristocétine (VWF:Rco) inférieur à $5 \%$ (norm $>50 \%$ ), un dosage antigènique (VWF : $\mathrm{Ag})$ inférieur à $1 \%$ (norm $>50 \%$ ). L'analyse moléculaire du gène du VWF a montré une délétion homozygote de 11 pb dans l'exon 20 qui enfant. Le père présente un phénotype biologique et clinique strictement normal et n'est pas porteur de la délétion alors que la mère présente la délétion à l'état hétérozygote avec un phénotype clinique normal et un phénotype biologique discrètement inférieur à la normale : VWF :Rco égal à $55 \%$ et VWF:Ag à $47 \%$. L'analyse de la famille à l'aide de 8 marqueurs génétiques polymorphes de la région a montré une unique contribution maternelle du chromosome $12 \mathrm{chez}$ la patiente et par PCR quantitative, la présence de deux allèles chez cette enfant. Ces résultats vont dans le sens d'une homozygotie de cette délétion par isodisomie maternelle de cette région du chromosome 12 . Les disomies parentales sont des anomalies génétiques bien connues dans certaines pathologies soumises à l'empreinte parentale mais aussi plus épisodiquement dans des maladies récessives, cependant aucune description de disomie du chromosome 12 n'avait jusqu'alors été mise en cause dans la maladie de Willebrand.

Mots-clés: maladie de Willebrand de type 3, disomie uniparentale, délétion.

IP349. LA PREMIÈRE MUTATION DE GNRH1 À L'ORIGINE D'UNE FORME FAMILIALE D'HYPOGONADISME HYPOGONADOTROPE AVEC OLFACTION NORMALE

J. Bouligand $(1,2)^{*}$, C. Ghervan (4)*, J.A. Tello (5), S. Brailly-Tabard (1, 2), S. Salenave (3), P. Chanson (2, 3), M. Lombès (2), R.P. Millar (5), A. Guiochon-Mantel $(1,2)^{* *}$, J. Young $(2,3)^{* *}$

(1) Laboratoire de Génétique Moléculaire, Pharmacogénétique et Hormonologie, CHU de Bicêtre (AP-HP), Le Kremlin Bicêtre, France ; 2) Université Paris-Sud, Faculté de Médecine Paris-Sud, INSERM U693, Le Kremlin Bicêtre, France ; 3) Service d'Endocrinologie et des Maladies de la Reproduction et Centre de Référence des Maladies Endocriniennes Rares de la Croissance, CHU de Bicêtre (AP-HP), Le Kremlin Bicêtre, France ; 4) Endocrinology Department, University of Medicine and Pharmacy Iuliu Hatieganu, Cluj Napoca 40349, Romania ; (5) Medical Research Council Human Reproductive Sciences Unit, The Queen's Medical Research Institute, Edinburgh, United Kingdom

* contributions équivalentes. ** contributions équivalentes de ces deux séniors

Contact : anne.mantel@bct.aphp.fr

Les hypogonadismes hypogonadotrophiques isolés congénitaux normosmiques (HHC) sont liés à un déficit de sécrétion de la GnRH hypothalamique ou à une absence de réceptivité à ce neuropeptide au niveau hypophysaire. Ils peuvent être associés à des mutations du récepteur de la $\mathrm{GnRH}$ (RGnRH), du récepteur de Kiss-1 (GPR54), de la neurokinine B (TAC3) ou de son récepteur (TACR3). Nous avons recherché des mutations du gène GNRH1, codant le prépro-GnRH, chez 200 patients atteints d'HHC ne présentant pas de mutation de RGnRH ni de GPR54. Dans une famille, nous avons identifié chez un frère et sa sœur atteints d'une forme sévère d'HHC une mutation de GNRH1 (c.18-19 insA) à l'état homozygote. Les parents non atteints, ainsi qu'une sœur non atteinte, sont porteurs hétérozygotes de cette mutation. Cette mutation entraîne un décalage du cadre de lecture à partir du $7^{c}$ acide aminé du signal peptide et la formation d'un peptide aberrant, ne contenant ni la séquence du décapeptide GnRH ni du GAP (GnRH associated peptide). L'absence d'immuno-réactivité à la GnRH dans des cellules exprimant des pro-convertases (AtT20) surexprimant ce mutant a été validée in vitro. L'administration pulsatile de GnRH à la patiente a permis de restaurer la pulsatilité de la LH confirmant l'origine hypothalamique de 1'HHC. Cette nouvelle forme génétique d'HHC familial permet de valider définitivement le rôle capital de la GnRH dans la reproduction humaine et de réfuter le rôle du GAP comme inhibiteur de la fonction lactotrope. II s'agit de la première description d'une mutation dans un facteur hypothalamique hypophysiotrope.

Mots-clés: GnRH, mutation, hypogonadisme hypogonadotrope.

IP350. MOLECULAR ANALYSIS OF THREE GENES IN ISOLATED HYPOPARATHYROIDISM

C. Silve (1, 2), G. Bertrand (2), A. Lienhardt (3), A. Linglart (1, 4), B. Grandchamp (2)

(1) INSERM U561, Hôpital St-Vincent-de-Paul, Paris, France ; (2) Service de Biochimie Hormonale et génétique, Hôpital Bichat, Paris, France ; (3) Pédiatrie Médicale, Hôpital Dupuytren, Limoges, France; (4) Service d'endocrinologie pédiatrique, Hôpital St Vincent de Paul, Paris, France Contact : Caroline.Silve@inserm.fr

Hypoparathyroidism (HP) is characterized by hypocalcemia, hyperphosphatemia, and absent, or markedly reduced serum PTH levels. Most cases of $\mathrm{HP}$ are sporadic, but familial forms (FHP) with either autosomal dominant (AD) or recessive (AR) inheritance are described. Syndromic and isolated forms exist. Isolated HP (IHP) are rare and have been shown to 
be caused by mutation in either of three genes critical for appropriate PTH secretion. Rare mutations in the PTH gene can cause AD or AR FIHP. More commonly, gain of function mutations in the CaSR gene have been identified in AD hypocalcemia. More recently, homozygous mutations in glial cells missing B (GCMB) have been reported in AR-IHP. Here we report the prevalence of mutations in the PTH, CaSR, and GCMB genes in a series of patients with familial or sporadic IHP collected from 2003 to 2009.

We obtained blood samples from 28 affected members of 13 IHP kindreds, all compatible with an $\mathrm{AD}$ transmission, and from 56 patients affected with sporadic IHP. In addition, we collected blood samples from 16 and 13 unaffected relatives from familial and sporadic cases respectively. When a new mutation was identified, its presence was searched for in genomic DNA from at least 48 healthy Caucasians. Sequence of all coding exons and intron-exon junctions for the CaSR, PTH, and GCMB genes were amplified and sequenced. Results showed:

PTH gene: no mutation.

CaSR gene: 7 heterozygous missense mutations, 6 in AD-HP and 1 in sporadic IHP. One mutation was reported by us (Silve, 2005), three are unpublished.

GCMB gene: (a) in AD-HP, 1 missense mutation previously described as autosomal recessive (R47L), and two heterozygous single base deletion leading to frame shifts (reported by us, Mannstadt, 2008). (b) In sporadic cases, one homozygous 9 bp insertion, one homozygous missense mutation and one heterozygous missense mutation, which, interestingly, also affects R47 (all unpublished).

No mutation in either of the 3 studied genes were identified in the remaining $4 \mathrm{AD}$-HP kindreds, and 52 sporadic cases.

Four major conclusions can be drawn from these results:

- Gene mutation in either CaSR or GCMB genes are more frequently identified in FIHP than in sporadic cases.

- AD-IHP can be caused by mutations in the GCMB gene with variable penetrance.

- For some GCMB mutations described as recessive, a phenotype can be observed at the heterozygous state.

- Most cases of sporadic IHP remain unexplained.

IP351. SOX3 ANALYSIS IN A LARGE COHORT OF PATIENTS WITH SYNDROMIC AND NON SYNDROMIC HYPOPITUITARISM : ALLELIC AND PHENOTYPIC HETEROGENEITY FUNCTIONAL CONSEQUENCES

M.L. Sobrier (1), I. Giurgea (1, 3), K. Machinis (1), M. Legendre (1, 2), M.P. Vie-Luton (1), S. Rose (2), N. Collot (2), G. Pinto (4), B. Mignot (5), A.M. Bertrand (5), C. Naud-Saureau (6), F. Kurtz (7), S. Odent (8), F. M'Bou (9), S. Cabrol (10), M. Houang (10), J.C. Carel (11), J. Leger (11), P. Czernichow (11), S. Amselem $(1,2)$

(1) INSERM U.933, Université Paris 6, Hôpital Armand-Trousseau, Paris, France ; (2) AP-HP, Service de Génétique et d'Embryologie médicales, Hôpital Armand-Trousseau, Paris, France; (3) AP-HP, Service de Biochimie et Génétique, Groupe Henri Mondor-Albert Chenevier, Créteil, France; (4) AP-HP, Service d'Endocrinologie, Hôpital Necker Enfants Malades, Paris, France; (5) Service d'Endocrinologie, Hôpital Saint Jacques, Besançon, France; (6) Service de Pédiatrie, Hôpital de Lorient, France; (7) Service de Pédiatrie, Hôpital de Saint-Avold, France ; (8) Service de Génétique CHU de Rennes, France ; (9) Service de Pédiatrie et néonatologie, CH du Lamentin, Martinique, France ; (10) AP-HP, Service d'explorations fonctionnelles Endocriniennes, Hôpital Trousseau, Paris, France; (11) AP-HP, Service d'Endocrinologie, Hôpital Robert Debré, Paris, France

Contact : marie-laure.sobrier@inserm.fr

SOX3, a transcription factor belonging to the SRY related high mobility group (HMG) box (SOX) family of proteins, is involved in the early control of pituitary development and function. So far, only 4 mutations have been identified in the SOX3 gene, which maps to Xq27.1. To assess SOX3 involvement in human pathology, we investigated 168 male patients from 166 independent families with pituitary dysfunction and with or without midline central nervous system (CNS) defects. Mutations were identified in 13 patients from 11 unrelated families. Expansions of the polyalanine tract ( +8 and +11 Ala) were found in 4 boys (from 2 families); 3 of them displayed isolated growth hormone deficiency (IGHD), whereas one had combined pituitary hormone deficiency (CPHD) involving GH and TSH. A contraction of the polyalanine tract $(-9 \mathrm{Ala})$ was found in a patient with CPHD and callosal anomalies. Missense mutations were observed in 8 independent patients : p.Ala102Ser in a patient with transitory ACTH deficiency, blindness related to septo-optic dysplasia and severe midline CNS anomalies; p.Arg5Pro in one with IGHD and behaviour troubles:
p.Arg5Gln in a patient with CPHD and mental retardation ; p.Pro3Ser in a patient with CPHD and hypertelorism ; and p.Prol03Thr in 4 independent patients with non-syndromic CPHD. A majority of patients had morphological pituitary anomalies: anterior pituitary hypoplasia (9/13), abnormal pituitary stalk (9/13), and ectopic posterior pituitary (10/13). The impact of each identified mutation on the subcellular location of SOX3 and its ability to transactivate a target construct were assessed through in vitro expression studies (i.e use of SOX 3 antibody and luciferase tests). The missense mutations and the contraction of the polyalanine tract (-9Ala) do not modify the expression pattern of this nuclear protein ; however, expansions of the poly-alanine tract $(+8$ or $+11 \mathrm{Ala})$ lead to the formation of SOX3 aggregates in the nucleus or both in the nucleus and the cytoplasm. Depending on the type of mutation, transcriptional activity was found to be diminished (+11Ala), increased (p.Pro3Ser, p.Arg5Pro, p.Pro103Thr, -9Ala) -in keeping with the previously described critical importance of SOX3 gene dosage in proper development-, or normal (+8Ala, p.Arg5Gln, p.Ala102Ser, therefore suggesting that these latter two variations may represent polymorphisms). This study, which identifies 8 SOX 3 mutations (among which 4 are new) in $7 \%$ of this large cohort, broadens the clinical spectrum of SOX3-related disorders.

Mots-clés : pituitary deficiency, SOX 3 mutations.

\section{P352. PARTIAL PROHORMONE CONVERTASE 1 DEFICIENCY CONFERS INCREASED RISK OF OBESITY}

H. Choquet (1), J.W.M. Creemers (2), M. Pigeyre (3), V. Vatin (1), S. Beckers (4), S. Meulemans (2), N. Potoczna (5), M. Tauber (6), B. Balkau (7), P. Elliot (8), M.R. Jarvelin (8, 9), F. Horber (5), W. Van Hul (4), L. Van Gaal (10), F. Pattou (11), P. Froguel (1, 12), D. Meyre (1)

(1) CNRS-8090-Institute of Biology, Pasteur Institute, Lille, France; (2) Department of Human Genetics, University of Leuven, Leuven, Belgium ; (3) Department of Nutrition, Hospital University, Lille, France; (4) Department of Medical Genetics, University of Antwerp and Antwerp University Hospital, Antwerp, Belgium; (5) Department of Surgery and Internal Medicine, Clinic Lindberg, medical departement, Winterthur and University of Berne, Switzerland; (6) Institut National de la Santé et de la Recherche médicale U563, Children's Hospital, Centre Hospitalier Universitaire de Toulouse, France; (7) INSERM U780-IFR69; University Paris Sud, Villejuif, France ; (8) Department of Epidemiology and Public Health, Imperial College London, London, United Kingdom ; (9) Institute of Health Sciences, University of Oulu Department of Child and Adolescent Health, National Public Health Institute, Biocenter Oulu, University of Oulu, Oulu, Finland; (10) Department of Endocrinology, Diabetology and Metabolism, Antwerp University Hospital, Antwerp, Belgium; (11) Institut National de la Santé et de la Recherche Médicale U859, Centre Hospitalier Régional Universitaire de Lille, Lille North of France University, Lille, France ; (12) Department of Genomic Medicine, Hammersmith Hospital, Imperial College London, London, UK

Contact : helene@good.ibl.fr

Complete prohormone convertase 1 (PCSK1) deficiency is a rare recessive form of obesity with only three cases reported so far. Although these homozygous or compound heterozygous carriers of loss of function mutations have severe phenotypes, including obesity, and intestine and hormonal defects, heterozygous family carriers were considered clinically unaffected. We sequenced PCSK1 exons in 845 non-consanguineous obese subjects of European origin. We characterized the functional consequences of the detected mutations on $\mathrm{PC} 1 / 3$ protein using in silico and in vitro studies. We also genotyped 6,104 obese and 6,274 non obese subjects for the identified functional mutations, to find additional carriers. Eight individuals $(0.95 \%)$ were heterozygous for novel PCSK1 non-synonymous mutations. No homozygous or heterozygous compound for a pathogenic mutation was found. Although in silico analyses predicted only $62.5 \%$ of these mutations to be potentially deleterious, we found that $87.5 \%$ of mutations had indeed an effect on $\mathrm{PC} 1 / 3$ processing and/or on its enzymatic activity. In addition, mutation $\mathrm{T} 175 \mathrm{M}$ located on a potential $\mathrm{N}$-glycosylation site of $\mathrm{PC} 1 / 3$ altered maturation and secretion of the protein. The genotyping of the seven pathogenic mutations in additional obese and normal weight subjects identified twelve carriers amongst cases and two carriers amongst controls. These data evidence a 6 -fold enrichment of these PCSK 1 mutations in European obese adults and children (Fisher's exact test, $\mathrm{P}=0.007$ ). Currently, family studies are undertaken to examine cosegregation of identified mutations with obesity and detailed phenotypic studies of heterozygous carriers and family members are in progress. In conclusion, in addition to the very rare syndromic forms of obesity due to complete PCSK1 deficiency, and to the frequent coding non-synonymous polymorphisms that modestly 
increase obesity risk, we now report that partial prohormone convertase 1 deficiency conferred by heterozygous non-synonymous mutations associates with obesity.

Mots-clés : prohormone convertase 1, monogenic, obesity.

口P353. ÉTUDE DE 109 CAS D'HYPERINSULINISME CONGÉNITAL RÉSISTANT AU DIAZOXIDE, AGONISTE DU CANAL KATP : SPECTRE MUTATIONNEL, CORRÉLATION AVEC LE DIAGNOSTIC HISTOPATHOLOGIQUE

C. Saint-Martin (1), C. Vaury (1), M. Santiago-Ribeiro (2), J.B. Arnoux (3), V. Verkarre (4), S. Gobrecht (1), F. Jaubert (4), Y. Aigrain (5), P. de Lonlay (3), C. Bellanné-Chantelot (1)

(1) Département de Génétique, AP-HP Groupe Hospitalier Pitié-Salpétrière, Université Pierre-et-Marie-Curie, Paris, France ; (2) Département de Recherche Médicale, Commissariat à l'Énergie Atomique, Orsay, France; (3) Centre de Référence des Maladies Métaboliques, AP-HP Hôpital Necker-Enfants Malades, Université Paris Descartes, Paris, France ; (4) Département d'Anatomo-pathologie, AP-HP Hôpital NeckerEnfants Malades, Université Paris Descartes, Paris, France ; (5) Département de Chirurgie pédiatrique, AP-HP Hôpital Necker-Enfants Malades, Université Paris Descartes, Paris, France Contact : cecile.saint-martin@psl.aphp.fr

L'hyperinsulinisme congénital caractérisé par une secrétion inappropriée d'insuline par les cellules bêta-pancréatiques est la principale cause d'hypoglycémie chez le jeune enfant. Une prise en charge rapide et adaptée est primordiale pour éviter toute complication neurologique. Celle-ci dépend de la réponse du patient au diazoxide et du diagnostic histopathologique. La forme focale correspond à une hyperplasie limitée à quelques ilôts de Langherans et est guérie par une pancréatectomie ciblée de la lésion alors que la forme diffuse est une hyperplasie de l'ensemble des ilôts de Langherans et nécessite une pancréatectomie subtotale. Les formes résistantes au diazoxide sont majoritairement associées à des mutations des gènes $\mathrm{ABCC} 8 \mathrm{et} \mathrm{KCNJ} 11$ codant pour les sous-unités SUR1 et KIR6.2 du canal potassique sensible à l'ATP (KATP) qui régule la secrétion d'insuline par les cellules bêta du pancréas. Nous avons criblé ABCC 8 et KCNJ11 par séquençage et par dosage génique (MLPA, QMPSF) pour 109 cas d'hyperinsulinisme congénital résistants au diazoxide. Les résultats ont ensuite été analysés en fonction du diagnostic histopathologique obtenu par examen radiologique (PET-CT) ou après chirurgie. Une anomalie moléculaire des gènes $\mathrm{ABCC} 8$ ou KCNJ11 a été identifiée dans $82 \%$ des cas. Parmi 119 mutations identifiées, 94 étaient distinctes et $50 \%$ d'entre elles étaient privées soulignant la diversité allélique. Dans $94 \%$ des cas, il s'agissait d'une mutation $\mathrm{ABCC} 8$. Tous les patients avec une forme focale étaient associés à une anomalie moléculaire du canal KATP. Les formes diffuses étaient génétiquement plus hétérogènes : $41 \%$ des patients étaient homozygotes ou hétérozygotes composites, $32 \%$ portaient une mutation hétérozygote et $27 \%$ étaient sans mutation. De façon intéressante, nous avons observé une transmission paternelle pour deux tiers des patients diagnostiqués avec une forme diffuse et portant une mutation hétérozygote du canal KATP. Le mécanisme de disomie uniparentale observé dans les formes focales a été exclu. Les gènes $\mathrm{ABCC} 8$ et KCNJ11 étant localisés à proximité de la région 11 p15.5 soumise à empreinte, nous avons recherché un défaut épigénétique reponsable de l'expression du seul allèle paternel. Nous avons exclu une anomalie de méthylation au niveau leucocytaire, une analyse au niveau pancréatique est en cours. Cette étude montre l'intérêt du diagnostic moléculaire dans les formes diffuses avec 2 mutations pour lesquelles un PET-CT n'est alors pas nécessaire et un conseil génétique envisageable pour la famille. Dans le cas des patients avec une seule mutation du canal KATP, l'analyse moléculaire doit être confrontée à l'exploration radiologique par PET-CT de façon à classer les patients en formes focales ou diffuses et à leur fournir une prise en charge clinique adaptée.

Mots-clés : hyperinsulinisme, ABCC8, KCNJ11.

PP354. INTÉRÊT DU TEST FONCTIONNEL EX VIVO D'ÉPISSAGE POUR LE DIAGNOSTIC MOLÉCULAIRE DES MALADIES GÉNÉTIQUES : ÉVALUATION DE L'EFFET DES SUBSTITUTIONS NUCLÉOTIDIQUES DE SIGNIFICATION INCONNUE

P. Gaildrat (1), A. Killian (1), J.C. Thery (1), M. Vezain (1), I. Tournier (1), S. Baert-Desurmont (1, 2), S. Krieger (3), A. Hardouin (3), T. Frébourg $(1,2)$, A. Martins (1), M. Tosi (1)

(1) Inserm U614, Faculté de Médecine, Insitut Hospitalo-Universitaire, Université de Rouen, Cancéropôle Nord-Ouest, France ; (2) Département de Génétique, Centre Hospitalo-universitaire de Rouen, France ; (3) Laboratoire de Biologie Clinique et Oncologique, Centre François Baclesse, Caen, Cancéropôle Nord-Ouest, France

Contact : mario.tosi@univ-rouen.fr

De nombreuses variations nucléotidiques de signification biologique maladies à déterminisme mendélien. L'interprétation de ces VSI représente un défi majeur en génétique médicale et, plus particulièrement, en oncogénétique. De nombreuses études ont montré qu'une fraction importante de ces VSI peut être pathogène du fait de l'altération du processus d'épissage de l'ARN pré-messager. Depuis 2007, nous avons introduit dans le cadre du diagnostic moléculaire un test fonctionnel d'épissage basé sur l'utilisation d'un minigène, permettant de déterminer si le VSI analysé est responsable d'un défaut d'épissage potentiellement délétère. Les principaux avantages de cet essai sont, d'une part, l'utilisation d'un matériel biologique toujours disponible, l'ADN génomique du patient et, d'autre part, une interprétation facilitée des résultats, notamment du fait de son caractère monoallélique. Nous avons mis à profit ce test dans le cadre d'une étude nationale portant sur 150 VSI identifiés dans les gènes MLH1 et MSH2, impliqués dans les cancers héréditaires du colon (Tournier et al., 2008 et données non publiées). Ces données ont contribué au reclassement de $19 \%$ des variants en mutations délétères d'épissage. L'utilisation de ce test nous a également permis d'analyser, dans le cadre d'une collaboration au sein du Cancéropôle Nord-Ouest, 73 VSI des gènes BRCA1 et BRCA2 impliqués dans les cancers héréditaires du sein et de l'ovaire et de reclasser $12 \%$ de ces variants en mutations d'épissage (Bonnet et al., 2008; Thery et al., données non publiées). La bonne concordance entre les données obtenues à l'aide du test d'épissage et celles établies par l'analyse RT-PCR de l'ARN des patients démontre la pertinence de cette approche. Aujourd'hui, compte-tenu du problème que posent les VSI dans le diagnostic moléculaire des maladies génétiques, nous avons développé au sein de notre unité Inserm cette activité de test d'épissage, sous forme de prestation de service que nous proposons aux laboratoires de génétique moléculaire afin de les aider dans l'interprétation de ces variants. Dans le cadre du diagnostic, cet essai représente i) une méthode alternative pour l'évaluation des effets des VSI sur l'épissage, quand l'ARN du patient n'est pas disponible, ii) une méthode de criblage, précédant la demande du prélèvement sanguin du patient pour la confirmation sur l'ARN, iii) une approche complémentaire à l'analyse de l'ARN du patient, quand ce matériel est disponible.

Mots-clés : variants de signification inconnue, test fonctionnel d'épissage, diagnostic moléculaire.

\section{aP355. ACTIVATION D'UN PSEUDOEXON ASSOCIÉE À UNE DOUBLE DÉLÉTION DANS UN INTRON DU GÈNE DMD}

M. Messaoud Khelifi (1, 2), C. Saquet (3), D. Thorel (3), D. Méchin (3), M. Claustres (1, 2, 3), P. Khau van Kien (3), S. Tuffery-Giraud (1, 2) (1) Université Montpellierl, UFR médecine, Montpellier, France; (2) INSERM, U827, Montpellier, France : (3) CHU Montpellier, Laboratoire de Génétique Moléculaire, Montpellier, France

Contact : mouna.messaoud-khelifi@inserm.fr

Les pseudoexons (PEs) sont des séquences introniques présentant toutes les caractéristiques des « vrais » exons (longueur, présence de sites accepteur et donneur d'épissage), bien que n'étant pas reconnues au cours du processus d'épissage. Cette régulation négative s'exercerait via un enrichissement en séquences inhibitrices d'épissage (exonic splicing silencers «ESS », intronic splicing silencers «ISS »), ou la présence de structure secondaire inadaptée de l'ARN pré-messager, ne permettant pas au spliceosome d'accéder aux sites d'épissage. L'exonisation de ces séquences introniques résulte le plus souvent du renforcement d'un des sites d'épissage par une mutation ponctuelle. L'étude des transcrits du gène DMD chez un patient atteint de Dystrophie Musculaire de Becker a révélé l'inclusion d'un PE de $166 \mathrm{pb}$ entre les exons 56 et 57 dans une fraction des transcrits musculaires (dp $427 \mathrm{~m})$, correspondant à une séquence de l'intron 56. L'analyse de l'ADN génomique chez ce patient n'a pas mis en évidence de mutation ponctuelle, mais la présence exceptionnelle de deux délétions génomiques, de 592 pb et 29 pb en 5'et 3 'du PE. Afin d'explorer le rôle de ces délétions dans l'activation du PE, nous avons construit une série de minigènes rapporteurs d'épissage correspondant à la séquence sauvage du PE et de ses bordures introniques (contexte sauvage), ou à la séquence du $\mathrm{PE}$ incluant les deux ou une seule des deux délétions en amont et en aval du PE. Les expériences d'épissage ex vivo ont permis de reproduire le profil anormal d'épissage en contexte muté, et de démontrer que seule la délétion en amont était nécessaire à l'activation du PE. Des études complémentaires sont en cours combinant une analyse bioinformatique à l'aide des logiciels Human Splicing Finder (HSF) et MFOLD et une approche de mutagénèse dirigée afin d'identifier les éléments cis régulateurs d'épissage (motifs de liaison à des protéines activatrices ou inhibitrices, et structure secondaire de l'ARN) potentiellement impliqués dans la reconnaissance de ce PE dans le gène DMD. Ces résultats démontrent que les remaniements génomiques dans les introns peuvent conduire à des évènements indésirables dans les transcrits tels que l'insertion de PE. 
Une meilleure connaissance des PEs du gène DMD et des éléments cisrégulateurs impliqués dans leur reconnaissance est essentielle pour le développement et la sécurité des thérapies basées sur le saut d'exon.

Mots-clés : pseudoexon, gène DMD.

DP356. IDENTIFICATION DE NOUVELLES SÉOUENCES DANS LES GËNES LAMA2 ET SGCG MODULANT LES ÉLÉMENTS RÉGULATEURS DE L'ÉPISSAGE EN CIS AINSI QUE LA STRUCTURE SECONDAIRE DE L'ARN MESSAGER

O. Siala, I. Hadj-Salem, A. Tlili, I. Ammar, H. Belguith, F. Fakhfakh

Laboratoire de Génétique Moléculaire Humaine, Faculté de Médecine de Sfax, Tunisie

Contact : olfa_siala@yahoo.fr

L'épissage de l'ARN pré messager nécessite la présence de sites et de consensus d'épissage par la machinerie cellulaire. En effet, et en plus des informations situées au niveau des sites 5 ' et 3 ' d'épissage, la cellule a besoin d’autres séquences activatrices ou inhibitrices de l'expression génique qui sont respectivement les enhancers et les silencers. Au cours de notre étude, nous avons séquencé les gènes LAMA2 et SGCG respectivement responsables des formes MDC1A et LGMD2C de dystrophies musculaires. Des études bioinformatiques ont été ensuite réalisées utilisant le programme ESEfinder pour la caractérisation des enhancers, ainsi que le programme MFOLD pour étudier l'effet des nouvelles variations nucléotidiques sur la structure secondaire de l'ARNm. Le séquençage a montré la présence de nouveaux polymorphismes dans le gène LAMA2 et SGCG qui n'existent pas dans la dernière version des banques de données. II s'agit des polymorphismes c.3174+22_23insAT et c.6085+12delA respectivement au niveau des introns 22 et 42 du gène LAMA2; ainsi que du polymorphisme $\mathrm{c} .{ }^{*} 102 \mathrm{~A} / \mathrm{C}$ dans la région $3^{\prime}$ UTR du gène SGCG. Ces variations ont été retrouvées à l'état homozygote chez 210 individus issus de 5 populations méditerranéennes (Tunisienne, Marocaine, Algérienne, Libanaise et Française). Le programme ESEfinder a montré que la variation c.*102A/C crée un enhancer qui se lie à la protéine SR «SF2/ASF " avec un score de 3.9 largement supérieur à la valeur du seuil significatif (1.9). Pour le gène LAMA2, l'ESEfinder a montré que la variation c. $3174+22$ 23insAT n'a pas d'effet sur la composition en enhancers ; alors que le polymorphisme c. $6085+12$ delA se trouve dans la région de liaison entre le consensus d'épissage en 5' et la protéine U1snRNA du spliceosome. Le programme MFOLD a montré que c.*102A/C est accompagné de plusieurs variations structurale touchant la position, le nombre et l'orientation des boucles externes. Cependant, le polymorphisme c.3174+22_23insAT n'a pas d'effet sur la structure secondaire de l'ARN ; alors que c. $6085+12$ delA a un effet remarquable sur la totalité de la structure de l'ARNm. Ces résultats montrent une corrélation structure-fonction entre la séquence primaire de l'ARN et la liaison aux facteurs d'épissage.

Mots-clés : épissage de l'ARNm, ESEfinder, MFOLD.

PP357. ANOMALIES D'ÉPISSAGE DUES À DES VARIANTS DE
SÉQUENCE NON CLASSÉS DANS LES GÊNES DES CAVERNO-
MATOSES CÉRÉBRALES ; UTILISATION DES LOGICIELS PRÉMATOSES CÉRÉBRAL
DICTIFS D'ÉPISSAGE

F. Riant (1, 2, 3), M. Cecillon (1), E. Tournier-Lasserve (1, 2, 3)

(1) Laboratoire de Génétique moléculaire, Hôpital Lariboisière, Paris, France ; (2) INSERM U 740, Paris, France ; (3) CERVCO, APHP, Hôpital Lariboisière, Paris, France

Contact : florence.riant@lrb.aphp.fr

Introduction : De nombreux variants non classés ont un effet sur l'épissage de l'ARNm par destruction ou création de séquences consensus d'épissage. Dans les Cavernomatoses Cérébrales Multiples (CCM), la majorité des mutations aboutit à l'apparition d'un codon stop prématuré et pour tout variant inconnu une conséquence sur l'épissage doit être suspectée. Objectifs : 1) rechercher les conséquences sur l'ARN de variants non classés 2) comparer les conséquences observées sue l'ARNm avec les prédictions données par les logiciels d'épissage. Méthode: les conséquences de 25 variants non classés trouvés chez des patients CCM ont été recherchées sur l'ARN de leucocytes périphériques. Un éventuel effet sur l'épissage du cDNA a aussi été recherché pour 11 mutations stop et 7 petites délétions ou insertions exoniques trouvées chez les patients CCM. Les résultats ont alors été comparés avec les prédictions données par 3 logiciels de prédiction d'épissage via Alamut. Résultats: 14 variants induisent un défaut d'épissage (10 introniques et 4 exoniques) et une mutation faux sens entraine un saut d'exon. Les 10 autres mutations stop et les 8 mutations de type insertion ou délétion ne modifient pas l'épissage. Treize des 15 variants altérant l'épissage étaient prédits comme délétères par les analyses in silico et 10 des 12 variants non classés sans effet sur l'épissage ont été correctement prédits comme neutres. Conclusion : 15 variants CCM affectent l'épissage, dont 3 mutations faux sens, une mutation silencieuse et une mutation stop. Les prédictions données par les logiciels étaient exactes dans $85 \%$ des cas pour un seuil de variation de $10 \%$ ( 2 faux positifs et 2 faux négatifs). Il est donc très important de tester la conséquence sur l'épissage pour tout nouveau variant trouvé dans les gènes CCM. Cependant, les prélèvements appropriés pour l'extraction d'ARN ne sont pas toujours disponibles et la présence de transcrits alternatifs peut être une gêne pour l'analyse. L'analyse par Alamut est une aide pour la prédiction les défauts d'épissage, mais les résultats doivent absolument être confirmés expérimentalement.

Mots-clés : variants non classés, logiciels prédictifs, cavernomatose cérébrale.

DP358. CONSÉQUENCES FONCTIONNELLES D'UNE MUTATION GERMINALE DU DOMAINE RICHE EN LEUCINE DE NLRP3, IDENTIFIÉE DANS UNE FORME ATYPIQUE DE MALADIE AUTOINFLAMMATOIRE - IMPLICATIONS THÉRAPEUTIQUES

I. Jéru (1, 2, 3), S. Marlin (4, 5), G. Le Borgne (1, 2), E. Cochet (3), P. Duquesnoy (1), F. Dastot-Le Moal (3), L. Cuisset (6), V. Hentgen (7), R. Dhote (8), G. Grateau (9), S. Amselem $(1,2,3)$

(1) INSERM, U933, Paris, France ; (2) Université Pierre-et-Marie-CurieParis6, UMR S 933, Paris, France: (3) Service de Génétique et Embryologie médicales, Hôpital Trousseau, Paris, France; (4) INSERM, U587, Paris, France ; (5) Centre de référence des surdités congénitales et héréditaires, Hôpital Trousseau, Paris, France: (6) Service de Biochimie et Génétique moléculaire, Hôpital Cochin, Paris, France; (7) Service de Pédiatrie, Centre Hospitalier de Versailles, Le Chesnay, France ; (8) Service de Médecine Interne, Hôpital Avicenne, Bobigny, France ; (9) Centre de référence Amyloses d'origine inflammatoire et Fièvre méditerranéenne familiale, Hôpital Tenon, Paris, France

Contact : isabelle.jeru@trs.aphp.fr

La physiopathologie des cryopyrinopathies, appartenant au groupe plus large des fièvres récurrentes héréditaires (FRHs), reste inexpliquée chez de nombreux patients. La quasi-totalité des anomalies moléculaires identifiées à ce jour sont localisées dans le domaine NBS (Nucleotide Binding Site) de la protéine NLRP3, codé par l'exon 3 du gène. Nous avons étudiế une forme familiale très atypique de cryopyrinopathie, de transmission autosomique dominante et présente sur trois générations, caractérisée par une surdité de perception, un syndrome inflammatoire biologique et une absence de signes cutanés. L'analyse de marqueurs microsatellites liés au gène NLRP3 nous a conduits à séquencer tous les exons de ce gène et à identifier une mutation faux-sens hétérozygote (p.Tyr859Cys), ségrégeant avec le phénotype pathologique. Cette mutation est localisée en C-terminal dans le domaine riche en leucine (LRR - leucin rich repeats) de la protéine. Des tests fonctionnels réalisés dans des cellules HEK293T, exprimant de manière stable ASC (Apoptosis-associated Speck-like protein containing a CARD) et la pro-caspase 1, ont montré un effet activateur modéré de cette mutation sur le clivage protéolytique de la pro-caspase 1 en caspase 1 et sur la formation de specks (agrégats intra-cytoplasmiques). En revanche, cette mutation n'a pas d'effet décelable sur la voie NFkB. Cette étude décrit la première forme familiale de cryopyrinopathie associée à une mutation de NLRP3 localisée hors de l'exon 3. Cela souligne l'importance de séquencer tous les exons de ce gène chez les patients présentant des manifestations atypiques, ce d'autant que les traitement anti-ILI sont très efficaces chez la quasi-totalité des patients présentant ce type d'affections. Par ailleurs, cette première étude des conséquences fonctionnelles d'une mutation faux-sens affectant le domaine LRR de NLRP3 montre à la fois l'intérêt et la limite des tests fonctionnels développés aujourd'hui pour tester la pathogénicité de nouveaux variants. À cet égard, l'effet « gain de fonction " de cette mutation sur la voie de signalisation de la pro-caspase 1 est en accord avec le phénotype inflammatoire des patients.

Mots-clés : autoinflammatoire, NLRP3, LRR.

IP359. PROFIL MUTATIONNEL DU GÈNE DE LA FIÈVRE MÉDITERRANÉENNE FAMILIALE AU MAROC

L. Belmahi (1), F.Z. Laarabi (1), I. Cherkaoui (1), A. Sefiani $(1,2)$

(1) Département de Génétique Médicale. Institut National d'Hygiène, Rabat, Maroc; (2) Centre de Génomique Humaine, Faculté de Médecine et de Pharmacie, Université Mohamed V, Rabat, Maroc

Contact : lati_belma@yahoo.fr

La Fièvre Méditerranéenne Familiale (FMF), ou maladie périodique, est une maladie héréditaire autosomique récessive. Elle se caractérise par la récurrence d'épisodes paroxystiques comportant une fièvre, une douleur abdominale, thoracique et/ou articulaire. La FMF touche surtout les 
populations du Bassin méditerranéen en particulier, les Arméniens, les Juifs Sépharades, les Turcs et les Arabes. La principale complication de cette maladie est une amylose généralisée, qui peut être évitée par un traitement à vie à la colchicine. Après avoir été pendant plusieurs années en diagnostic par élimination des autres causes de fièvres récurrentes, la confirmation diagnostique de la FMF est aujourd'hui moléculaire, basée sur la mise en évidence de mutations du gène MEFV qui a été cloné en 1997. Nous rapportons à travers cette étude la première série de mutations à l'origine de la FMF dans la population marocaine. Le séquençage de l'exon 10 et de l'exon 2 du gène MEFV, nous a permis d'identifier un panel de 7 mutations situées dans l'exon 10 et une seule mutation (E148Q) au niveau de l'exon 2. La mutation M694V et la mutation M694I sont majoritaires à $53 \%$ et $30,5 \%$ respectivement, avec une prévalence élevée de la M694V chez la population originaire de la ville Fès. Les autres mutations plus rares sont la E148Q (5,5\%), M680L (4,5\%), A744S $(4,5 \%)$, M694del $(1 \%)$, R761H (1\%).

Mots-clés : fièvre méditerranéenne familiale, diagnostic moléculaire, MEFV.

\section{aP360. DÉFICIENCE COGNITIVE ET PETITE TAILLE :} EXPRESSION INHABITUELLE D'UN SYNDROME CINCA

C. Beneteau (1), F. Guerin (1, 2), I. Lemelle (2), J. Gosselin (1), J.L. Schaeffer (3), K. Angioi (4), I. Touitou (5), B. Leheup (1)

(1) Centre de Référence Est Syndromes Malformatifs et Anomalies du Développement, Service de Médecine Infantile III et de Génétique Clinique CHU de Nancy et EA 4368 Faculté de Médecine Nancy-Université Université Henri Poincaré, Nancy, France ; (2) Service de Médecine Infantile II, CHU de Nancy, France; (3) Service de Pédiatrie, Hôpital de SaintDizier, France; (4) Département d'ophtalmologie, CHU de Nancy, France; (5) Unité Médicale des Maladies Auto-Inflammatoires, Hôpital A de Villeneuve, Centre Hospitalier Universitaire de Montpellier, Montpellier, France

Contact : leheup.bruno@wanadoo.fr

Parmi les fièvres intermittentes héréditaires familiales, le syndrome CINCA (Chronic Infantile Neurological Cutaneous and Articular) est une maladie rare, caractérisée par un début très précoce avec typiquement un érythème diffus durant la période néonatale et une triade clinique associant : manifestations cutanées, céphalées chroniques et arthropathies. Des observations « mild » du syndrome CINCA ont déjà été rapportées avec une révélation clinique plus tardive et des symptômes plus modérés, proches du syndrome de Muckle Wells. Nous rapportons une observation de syndrome CINCA, avec mutation identifiée dans le gène NLRP3-CIAS1 chez deux jumeaux monozygotes, dont le tableau clinique est inhabituel. Ils sont nés en 1996 , les $2^{\circ}$ et $3^{\circ}$ de leur père et $6^{c}$ et $7^{\circ}$ de leur mère. Aucun des deux parents n'a de qualification professionnelle. Un des deux jumeaux présentait un RCIU avec taille et poids à $-2,5$ DS. Les deux patients consultent initialement à l'âge de 8 ans pour des difficultés scolaires avec scolarisation en CLIS dans le cadre d'une déficience légère avec QI 55, QIV 62, QIP 58 chez l'un et QI 66, QIV 66, QIP 73 chez l'autre et un retard de croissance (tailles à respectivement $-2,5$ et $-3,5 \mathrm{DS}$ ). Le bilan hormonal ne met pas en évidence de déficit somatotrope. Le bilan inflammatoire systématique retrouve une VS augmentée à 30/60 chez les deux patients. La réévaluation étiologique retrouve en fait une surdité de perception bilatérale chez les deux enfants et la notion de deux épisodes de méningites aseptiques chez un des jumeaux. Un œdème papillaire bilatéral au fond d'œil est également retrouvé à l'âge de 9 ans dans le cadre du bilan d'un épisode aigu de céphalées et de vomissements. Au plan morphologique, les particularités sont discrètes avec front proéminent, antéversion narinaire et philtrum long. Malgré l'absence de signes articulaires (bilan radiologique articulaire normal) ou cutanés, la suspicion de syndrome CINCA est confirmée par la détection de la mutation c.2068G $>$ A dans le gène NLRP3. Les deux enfants sont traités depuis maintenant un an par Anakinra. Il faut souligner la reprise staturale nette puisque la vélocité de croissance annuelle préthérapeutique se situait à -3 ds et à +2 DS un an après l'initiation du traitement Nous rappelons au travers de cas deux observations que les syndromes inflammatoires chroniques peuvent être associés à des troubles cognitifs et soulignons l'intérêt de la mesure systématique de la VS dans les consultations génétiques de retard mental.

Mots-clés : CINCA, NLRP3, retard mental.

aP361. DÉLÉTIONS DANS LE DOMAINE C-TERMINAL DU GÈNE ALAS2 : NOUVELLE PORPHYRIE, NOUVEAU MÉCA. NISME MOLÉCULAIRE

S. Ducamp (1, 2), C. Martin-Schmitt (1), S. Lyoumi (1), C. Kannengiesser (1, 3), L. Gouya (1, 2), B. Grandchamp (1, 3), J.C. Deybach (1, 2), H. Puy (1, 2) (1) INSERM Unité 773, Centre de Recherche Biomedicale Bichat-Beaujon,
Français des Porphyries, Hôpital Louis Mourier, Colombes, France ; (3) AP-HP, Service de Génétique et Biochimie hormonale, Hôpital Bichat Claude Bernard, Paris, France

Contact : sarah.ducamp@inserm.fr

La protoporphyrie érythropoḯtique (EPP, MIM177000) est une maladie rare héréditaire résultant d'un déficit partiel de l'activité de la Ferrochélatase (FECH, EC.4.99.1.1), dernière enzyme de la voie de biosynthèse de l'hème. Ce déficit est responsable de l'accumulation de Protoporphyrine IX (PPIX) entraînant le développement d'une photodermatose algique et plus rarement, dans $2 \%$ des cas, d'une insuffisance hépatique terminale. Le mode de transmission original de cette maladie repose sur la co-transmission d'un allèle FECH portant une mutation délétère, en trans de l'allèle commun hypomorphe FECH IVS3-48C, ramenant l'activité FECH en dessous du seuil critique de $30 \%$ (Gouya et al., Am. J. Hum. Genet. 2006). Pour $7 \%$ des patients EPP, aucune mutation FECH n'est trouvée. Parmi ceux-ci $3 \%$ sont homozygotes pour l'allèle normal FECH IVS3-48T et présentent une activité FECH normale. Ceci suggère fortement l'existence d'un variant EPP impliquant un autre locus. Chacune des sept porphyries héréditaires résulte d'un déficit partiel d'une des enzymes de la voie de biosynthèse de l'hème. Avant ce travail, aucune mutation affectant les gènes ALAS1 et ALAS2, codant respectivement pour les isoformes ubiquitaires et érythroïdes spécifiques de l'acide delta-aminolévulinique synthétase (ALAS, EC 2.3.1.37), première enzyme de la voie de biosynthèse de l'hème, n'a été associée à une porphyrie. Les mutations affectant le gène ALAS2 sont responsables chez l'homme d'anémies sidéroblastiques liées à l'X (XLSA, MIM 301300). Nous décrivons ici huit familles EPP pour lesquelles le locus FECH a été exclu. Nous avons identifié chez les atteints des délétions dans le domaine C-terminal de l'ALAS2, soit c.1706-1709 delAGTG (p.E569GfsX24) ou c.1699-1700 delAT (p.M567EfsX2), menant à la délétion ou au remplacement des 19-20 derniers résidus de l'enzyme. Les études d'expression, en système procaryote, montrent une augmentation de l'activité ALAS2 pour les deux types de mutations. Ces mutations gain de fonction sont responsables d'une nouvelle forme de porphyrie : la protoporphyrie érythropoḯtique dominante liée à l'X (XLDPP, MIM 300752), biochimiquement caractérisée par une haute proportion de zinc-PPIX dans les érythrocytes (Ducamp et al., Am. J. Hum. Genet. 2008). De plus, nos études préliminaires indiquent que seule une partie définie du domaine C-terminal est impliquée dans l'augmentation de l'activité ALAS2. Enfin les enzymes mutants montrent une température optimale inférieure à celle de l'enzyme sauvage, suggérant une énergie d'activation plus faible entraînant le gain de fonction.

Mots-clés : protoporphyrie érythropoïétique, ALAS2, liée à l'X.

IP362. MUTATIONS DE TMPRSS6 AFFECTANT LES DIFFÉRENTS DOMAINES FONCTIONNELS DE LA PROTÉINE DANS DES ANÉMIES MICROCYTAIRES FERRIPRIVES

F. Guillem (1), C. Oudin (2), C. Kannengiesser (1, 2), P. Matak (3), G. Nicolas (3), J. Donadieu (4), B. Isidor (5), P. Aguilar-Martinez (6), S. Vaulont (3), C. Beaumont (1), B. Grandchamp $(1,2)$

(1) INSERM UMR773 et Université Paris Diderot, site Bichat, Pari, France ; (2) APHP, Hôpital Xavier Bichat, Service de Biochimie Hormonale et Génétique, Paris, France; (3) Institut Cochin, Université ParisDescartes, CNRS, UMR8104, INSERM U567, France ; (4) APHP, Service d'Hématologie Pédiatrique, Hôpital Armand-Trousseau, Paris, France ; (5) Service de Génétique Médicale, CHU de Nantes, Nantes, France ; (6) Service d'Hématologie Biologique, $\mathrm{CHU}$ de Montpellier, France

Contact : bernard.grandchamp@inserm.fr

TMPRSS6 est une sérine protéase membranaire d'expression hépatique qui réprime l'expression de l'hepcidine, hormone clé du métabolisme du fer. Cette régulation s'opère par la dégradation de la forme membranaire de l'hémojuvéline, corécepteur des BMPs indispensable à la régulation de l'hepcidine par le fer. TMPRSS6 comporte un court domaine intracytoplasmique, un domaine transmembranaire et un ectodomaine composé de 1 domaine SEA, 2 domaines CUB, 3 domaines LDLR et un domaine sérine protéase. Comme toutes les protéines appartenant à la famille des TTSP (type II transmembrane serine protease), la protéine TMPRSS6 est activée via un clivage autocatalytique après une arginine, en $\mathrm{N}$-term du domaine sérine protéase. Des mutations du gène TMPRSS6 ont été décrites chez des patients souffrant d'une anémie avec déficience en fer, réfractaire au traitement par le fer oral (IRIDA), une maladie transmise sur le mode autosomique récessif et caractérisée par un fer sérique bas, un faible pourcentage de saturation de la transferrine, une microcytose. La physiopathologie de la maladie s'explique par une concentration d'hepcidine plasmatique anormalement élevée conduisant à une hypoabsorption du fer alimentaire et une rétention du fer macrophagique. L'administration de fer par voie intraveineuse entraîne une augmentation de la ferritine sérique et 
une correction de l'anémie quasi complète et la réponse à ce traitement est généralement durable : la concentration d'hémoglobine est maintenue à des valeurs normales ou subnormales pendant plusieurs mois. Ces observations suggèrent que malgré une concentration plasmatique élevée d'hepcidine, une part du fer administré par voie intraveineuse et capté par les macrophages est lentement redistribuée dans la circulation puis utilisée pour l'érythropoìèse. Nous avons récemment identifié cinq nouvelles mutations chez 5 patients ( 3 cas index) par séquençage des 18 exons, des jonctions introns-exons, et du promoteur du gène. Deux frères étaient homozygotes pour la délétion d'un nucléotide dans l'exon 15 entrainant la perte du domaine sérine protéase (p.A605fsX8). Trois patients étaient hétérozygotes composites pour des mutations faux sens touchant différents domaines de la protéine: p.Glu114Lys localisée dans le putatif domaine SEA et p.Pro765Ala dans le domaine sérine protéase chez un patient, p.Leu235Pro dans le premier domaine CUB et p.Tyr418Cys dans le deuxième domaine CUB chez deux frères d'une autre famille. Les quatre acides aminés touchés par les mutations faux sens et localisés dans différents domaines de la protéine sont très conservés entre les espèces. L'étude fonctionnelle, par transfections dans les cellules $\mathrm{HuH} 7$, de cDNAs codant pour des protéines TMPRSS6 portant les mutations faux-sens dans les différents domaines montre que l'intégrité structurale de ces domaines est nécessaire au clivage autocatalytique activateur de TMPRSS6.

Mots-clés : hepcidine, IRIDA, TMPRSS6.

aP363. LA MUTATION V617F DU GÈNE JAK2 CHEZ LES MALADES DE SYNDROMES MYÉLOPROLIFÉRATIFS AU MAROC : CONTRIBUTION AU DIAGNOSTIC ET PERSPECTIVES THÉRAPEUTIQUES

H. Dehbi (1), A. Benmoussa (1), S. Fehri (2), A. Quessar (2), S. Nadifi (1) (1) Laboratoire de Génétique Médicale, Faculté de Médecine et de Pharmacie de Casablanca, Maroc; (2) Service d'hématologie et d'oncologie pédiatrique, hôpital 20 aout 1953, Casablanca, Maroc

Contact : hind500@yahoo.fr

La mutation JAK2 V617F est de découverte récente, son implication dans la pathogénie des syndromes myéloprolifératifs (SMP) est actuellement confirmée. Notre étude est la première à s'intéresser au statut de la mutation JAK2 V617F chez les patients souffrants d'un syndrome myéloprolifératif au Maroc. L'étude a porté sur 70 patients, La mutation a été détectée par PCR allèle spécifique (AS-PCR).L'incidence de la mutation V617F JAK2 dans la polyglobulie de vaquez, thrombocytémie essentielle et la myélofiborse idiopathique sont respectivement $89,47 \%, 62,5 \%$ et $33,33 \%$. Nous avons également constaté que les patients porteurs de cette mutation présentent des niveaux plus élevés de l'hémoglobine et l'hématocrite par rapport aux malades ne portant pas cette mutation. Par conte, la mutation V617F JAK2 est absente chez les patients atteints de polyglobulie ou de thrombocytose secondaire. Notre étude est la première à évaluer la mutation JAK2 V617F chez des patients avec syndromes myéloprolifératifs au Maroc. Nos données semblent confirmer que la mutation JAK2 V617F est spécifique aux syndromes myéloprolifératifs.

Mots-clés : syndromes myéloprolifératifs, mutation V617F, gène JAK2.

- P364. HÉTÉROGÉNÉITÉ GÉNÉTIQUE DES ANÉMIES SIDEROBLASTIQUES : SÉRIE DE 31 PROPOSANTS

C. Kannengiesser (1, 3), S. Ducamp (2, 3), G. Hetet (1), D. Henry (1), C. Oudin (1), M. Touati (4), L. Garçon (5), H. Puy (2, 3), C. Beaumont (3), B. Grandchamp $(1,3)$

(1) AP-HP, Service de Génétique et Biochimie hormonale, Hôpital BichatClaude-Bernard, Paris, France, Université Paris-Diderot, Paris, France, (2) AP-HP. Centre français de porphyries, Hôpital Louis-Mourier, France ; (3) INSERM U773, Université Paris-Diderot, Paris, France ; (4) service d'hématologie clinique, CHU de Limoges, Hôpital Dupuytren. Limoges, France; (5) AP-HP, service d'hématologie Biologique, Hôpital Hotel-Dieu, Paris

\section{Contact : bernard.grandchamp@inserm.fr}

L'anémie sidéroblastique (AS) se définit par la présence de sidéroblastes en couronne dans la moelle osseuse. Les formes génétiques d'AS sont soit isolées, soit syndromiques. À ce jour, 7 formes d'AS congénitales sont identifiées. Les formes isolées sont: l'AS liée à l'X impliquant le gène ALAS2 qui est la forme la plus fréquente, l'anémie autosomique récessive non syndromique (gène SLC25A38) et l'AS avec surcharge en fer (gène GLRX5). Quatre formes sont syndromiques : l'anémie mégaloblastique répondant â la thiamine, avec diabète et surdité (gène SLC19A2), l'anémie sidéroblastique liée à l' $\mathrm{X}$ et ataxie (gène $\mathrm{ABCB}$ 7), la myopathie mitochondriale, acidose lactique et AS (gène PUS1) et le syndrome de Pearson, cytopathie mitochondriale caractérisé par une AS et une insuffisance pancréatique. Nous avons reçu depuis 2002 des prélèvements pour 31 proposants ( 20 hommes et 11 femmes) pour lesquels a été établi un diagnostic d'AS sur la présence de sidéroblastes en couronne dans la moelle. En fonction du dossier clinico-biologique, une recherche de mutations dans les séquences codantes, les jonctions exons-introns et promoteurs des gènes ALAS2, SLC25A38, SLC19A2, GLRX5 a été réalisée par séquençage direct. Des mutations ont été identifićes dans 3 de ces gènes :-ALAS2: 16 cas index dont 11 hommes et 5 femmes (52\% des proposants) dont 7 nouvelles mutations. Toutes les femmes atteintes d'AS liées à l'X présentaient un biais d'inactivation de l'X. Ces mutations sont dans 6 cas des substitutions affectant des codons conservés et dans un cas une délétion au niveau du promoteur. L'expression des cDNAs mutés chez E coli confirme l'importance fonctionnelle des mutations faux sens observées. On observe une variabilité phénotypique importante chez les patients porteurs de mutation d'ALAS2, certains d'entre eux diagnostiqués à l'âge adulte présentent une microcytose associée à une surcharge en fer parfois importante mais une anémie très modérée ou même absente. -SLC25A38: 4 patients porteurs de mutations ( $13 \%$ des cas) dont 3 nouvelles mutations. Dans 3 cas sur 4 , il s'agissait d'homozygotie vraie, traduisant une consanguinité. -SLC19A2 : 1 patient porteur d'une mutation déjà décrite dans une autre famille. Cette série confirme la grande hétérogénéité génétique des AS. Les formes liées à l'X sont les plus fréquentes dans notre série ( $52 \%$ ). Cette proportion est proche de celle de $37 \%$ rapportée dans la plus grande série américaine publiée récemment (Bergmann et al., Pediatr Blood Cancer, 2009). L'absence d'AS liées à GLRX5 confirme leur rareté. Dans une proportion relativement élevée de patients aucune anomalie génétique n'a pu été identifiée ( $32 \%$ dans notre série, $43 \%$ dans la série américaine) suggérant l'implication d'autres gènes non identifiés à ce jour dans les AS.

Mots-clés : anémie sidéroblastique, ALAS2, SCL25A38.

nP365. MYÉLODYSPLASIE NÉONATALE: PREMIER SIGNE D'UNE INTOLÉRANCE AUX PROTÉINES DIBASIQUES

M. Barth (1), X. Rialland (2), S. Le Bouedec (3), G. Simard (4), M. Nagel (5), D. Bonneau (1)

(1) Service de génétique, CHU Angers, Angers, France; (2) Service d'onco-hématologie pédiatrique, CHU Angers, Angers, France ; (3) Service de néonatalogie, CHU Angers, Angers, France; (4) Service de biochimie métabolique, CHU Angers, Angers, France ; (5) Center for metabolic disorders, Weisswasser, Germany

Contact : mabarth@chu-angers.fr

Introduction : L'intolérance aux protéines dibasiques (LPI) est une maladie métabolique rare par mutation d'un transporteur des acides aminés dibasiques (lysine, ornithine, arginine) situé sur la membrane basolatérale des entérocytes, des cellules tubulaires rénales, des hépatocytes et de l'épithélium bronchique. Classiquement, la maladie débute après le sevrage par des vomissements, des diarrhées, un retard de croissance, une hépatosplénomégalie, une ostéoporose et une protéinose alvéolaire. Une atteinte hématologique à type de pancytopénie a également été rapportée avec parfois un début néonatal. Le myélogramme retrouvait alors toujours un syndrome d'activation macrophagique. Cas clinique : Nous rapportons le cas d'une enfant qui a développé à $48 \mathrm{~h}$ de vie, une thrombopénie sévère isolée à 20000 plaquettes $/ \mathrm{mm}^{3}$. L'enfant a régulièrement été transfusée en plaquettes. Le myélogramme a montré une moelle dysplasique avec myélofibrose réticulinique, érythoblastopénie et mégacaryocytopénie. À 6 mois, alors que la diversification alimentaire a été débutée, elle a présenté une diarrhée chronique entrainant une cassure de la courbe staturo-pondérale. Le bilan métabolique a retrouvé une élévation de la glutamine et des taux plasmatiques effondrés de lysine, arginine et ornithine alors que la chromatographie d'acides aminés urinaires était normale. Évolution: Malgré la normalité de l'excrétion urinaire d'acides aminés dibasiques, le diagnostic d'intolérance aux protéines dibasiques a été retenu et confirmé par la mise en évidence de deux mutations hétérozygotes dans le gène SLC7A7 codant pour le transporteur $\mathrm{y}+\mathrm{LAT} 1$ des acides aminés dibasiques. Un traitement par nutrition parentérale restreinte en protéines ainsi qu'une supplémentation en citrulline et carnitine a été débuté. Rapidement la diarrhée s'est estompée, la croissance staturo-pondérale a repris et les besoins transfusionnels ont progressivement diminués. À 18 mois, le myélogramme est normalisé. Les apports protidiques entéraux sont alors progressivement augmentés jusqu'à $2 \mathrm{~g} / \mathrm{kg} / \mathrm{j}$. À l'âge de 7 ans, le traitement par citrulline a été arrêté. Six mois plus tard, l'enfant a développé une nouvelle thrombopénie sévère. La reprise du traitement par citrulline et d'un régime végétarien apportant $1,5 \mathrm{~g} / \mathrm{kg} / \mathrm{j}$ a permis la normalisation de la numération formule sanguine en un an. Conclusion : Nous rapportons le cas d'une enfant qui a développé une myélodysplasie de début néonatal causée par une intolérance aux protéines dibasiques. La mise en place du traitement spécifique par citrulline et d'un régime restreint en protéines a permis une 
normalisation du bilan hématologique. Le diagnostic d'intolérance aux protéines dibasiques doit donc être évoqué comme diagnostic différentiel des myélodysplasies de révélation néonatale.

Mots-clés : myélodysplasie, intolérance aux protéines dibasiques.

IP366. UNE NOUVELLE MUTATION, P.ASN491SER, DU GÈNE CODANT DMT1 MODIFIE LE ROUTAGE DE LA PROTÉINE ET CONDUIT, LORSQU'ELLE EST ASSOCIÉE À LA MUTATION P.GLY212VAL, À UNE ANÉMIE MICROCYTAIRE HYPOCHROME E. Bardou-Jacquet (1, 2, 3), A. Mosser (2, 4), M.L. Island (3), L. Detivaud $(2,3)$, N. Fatih (3), E. Brissot (5), M. Ropert (2, 3, 6), H. Maisonneuve (5), P. Brissot (1, 2, 3), O. Loréal (2, 3), A.M. Jouanolle $(2,4)$

(1) Service des maladies du foie, CHU Pontchaillou, Rennes, France ; (2) Centre de référence des surcharges génétiques en fer rares, $\mathrm{CHU}$ Pontchaillou, France ; (3) Inserm U-522, Université de Rennes I, France ; (4) Laboratoire de génétique moléculaire, CHU Pontchaillou, Rennes, France; (5) Service de médecine interne et hématologie, $\mathrm{CH}$ les Oudairies, La Roche sur Yon, France; (6) Laboratoire de biochimie, CHU Pontchaillou, Rennes, France

Contact : anne-marie.jouanolle@chu-rennes.fr

DMT1 est une protéine transmembranaire assurant le transfert de cations divalents, dont le fer, du pôle apical vers le cytosol dans les entérocytes, et des endosomes acidifiés vers le cytosol dans les autres types cellulaires. Des mutations du gène SLC11A2 codant DMT1 conduisent à une anémie microcytaire hypochrome associée le plus souvent à une surcharge hépatique en fer massive. Nous rapportons ici le 5ème cas d'anémie microcytaire relevant du gène codant DMT1. Il s'agit d'une jeune femme chez laquelle le diagnostic d'anémie microcytaire hypochrome a été posé à l'âge de 13 ans $(\mathrm{Hb}: 8,6 \mathrm{~g} / \mathrm{dL}$; VGM : $58 \mathrm{fl}$; CHM : 17,8 pg/cell). À 27 ans, elle est adressée pour avis au Centre de Référence des surcharges génétiques en fer rares (Rennes). Le bilan hématologique est inchangé, le bilan martial perturbé révèle une franche augmentation du fer sérique et du coefficient de saturation de la transferrine (respectivement $37 \mu \mathrm{mol} / \mathrm{L}$ et $83 \%$ ) : la concentration hépatique en fer évaluée par IRM est très élevée $(300 \mu \mathrm{mol} / \mathrm{g}$ de poids $\sec (\mathrm{N}<36))$, contrastant avec une ferritinémie modérément augmentée $(214 \mu \mathrm{g} / \mathrm{L})$. L'analyse du gène SLC11A2 par séquençage a mis en évidence deux mutations hétérozygotes: la mutation p.Gly212Val déjà décrite (Beaumont et al., 2006) et la mutation p.Asn491Ser non rapportée dans la littérature. L'impact fonctionnel des mutations a été évalué sur i) l'épissage de l'ARN et ii) le routage de la protéine dans la lignée cellulaire humaine $\mathrm{HUH} 7$ d'origine hépatique en utilisant le vecteur d'expression pdsRed2-C1 dans lequel les formes sauvages et mutées de l'ADNc ont été insérées. Les résultats i) montrent l'impact, sur le métabolisme du fer, d'une hétérozygotie composite du gène codant DMT1 qui entraîne anémie microcytaire et surcharge en fer paradoxale dont le mécanisme reste à élucider, ii) rapportent une mutation p.Asn491Ser ayant un impact sur le routage cellulaire de la protéine, et iii) suggèrent que la mutation $\mathrm{p}$.Gly $212 \mathrm{Val}$ a un impact sur l'activité de la protéine sans affecter son routage cellulaire.

Mots-clés : DMT1, anémie, fer.

IP367. IMPACT DES MUTATIONS G1691A DU FACTEUR V LEIDEN ET DE G20210A DE LA PROTHROMBINE DANS LA GENÈSE DE LA MALADIE THROMBO-EMBOLIQUE

H. Dehbi (1), H. QAadda (1), K. Raddaoui (1), M. Miguil (2), S. Faiz (3), I. Slassi (4), S. Nadifi (1)

(1) Laboratoire de Génétique Médicale, Faculté de Médecine et de Pharmacie de Casablanca, Maroc; (2) Service de réanimation gynéco obstétricale, CHU Ibn Rochd, Casablanca, Maroc; ; (3) Laboratoire d'hématologie, CHU Ibn Rochd, Casablanca, Maroc; (4) Service de neurologie. CHU Ibn Rochd, Casablanca, Maroc

Contact : hind500@yahoo.fr

Les mutations du facteur V Leiden (G1691A), de la prothrombine(G20210A) sont fréquentes en Europe et rare en Afrique, cependant leur implication dans la genèse des complications thromboembolique est certaine. Le but de cette étude était de déterminer la prévalence de ces mutations chez des malades ayant eu différentes complications thromboemboliques afin de déterminer les causes génétiques prédisposant à la maladie thromboembolique (MTE). La recherche de ces mutations a concerné différentes complications thromboemboliques (AVC, TVP, complications obstétricales...) provenant de différents services de CHU Ibn Rochd de Casablanca. Notre méthodologie consiste en une amplification génique par PCR de l'ADN génomique, suivie d'un clivage enzymatique. Nos résultats ont montré que la mutation du FV Leiden est significativement plus élevée lors des complications obstétricales $(6,25 \%)$ alors que la mutation du facteur II a été retrouvée avec une prévalence de $4.7 \%$ chez les TVP contre $3,12 \%$ dans les complications obstétricales. Ces deux mutations étaient absentes lors des AVC, thrombophlébite. Ces résultats ont été comparés à ceux obtenus dans une autre étude réalisée préalablement chez 200 sujets sains pris au hasard dans la population Marocaine. Ces données révèlent que la prévalence de mutation du facteur V Leiden qui était absente chez la population saine est beaucoup plus impliquée dans la genèse des TVP que dans les complications obstétricales alors que le FII est impliqué à part égale et son taux avoisine celui retrouvé chez la population saine $(2,4 \%)$.

Mots-clés : maladie thromboembolique, mutation G1691A, mutation G20210A.

- P368. LE TAUX D'HÉMOGLOBINE A2 N'EST PAS TOUJOURS FACILE A INTERPRÉTER: LE CAS D'UNE HÉMOGLOBINE RÉSULTANT D'UN GENE DE FUSION AVEC PROMOTEUR BÊTA-GLOBINE

K. Moradkhani, C. Préhu, J. Riou, M. Mathis, C. Godart, M. Goossens, H. Wajcman

Laboratoire des hémoglobines, Service de Biochimie et Génétique, Hôpital Henri Mondor, Créteil, France

Contact : kamikimi@yahoo.com

Le taux d'hémoglobine A2 est diminué dans les alpha-thalassémies et augmenté dans les bêta-thalassémies. Le degré d'augmentation et/ou de diminution du taux d'HbA2 dépend de la nature de l'anomalie. Pendant la période embryonnaire et foetale, ce sont les gènes dzêta- et alpha-globine, sur le cluster alpha- et epsilon- et gamma-globine sur le cluster bêta, qui sont exprimés. Ce n'est qu'à partir de la 36ème semaine d'aménorrhée que les gènes de deltaet bêta-globine s'expriment. Cette régulation est sous le contrôle du LCR (cluster bêta) et de la région HS40 (cluster alpha). Le promoteur du gène delta-globine est faiblement stimulé par LCR : le taux d'HbA2 (alpha2delta2) se situe à $2.5 \%$ après l'âge d'un an et reste à ce niveau toute la vie. Au contraire, le promoteur de bêta-globine est rapidement fortement stimulé et le reste de l'Hb est constitué par de l'HbA (alpha2beta2).

Quelles sont les causes d'une $\mathrm{Hb} \mathrm{A} 2$ abaissée ? Ceci est fréquemment observé dans le cadre d'une carence martiale car le fer est impliqué dans la transcription de l'alpha-globine. La diminution d'HbA2 est également rencontrée dans les alpha-thalassémies. Une autre cause d'Hb A2 basse, sans traduction clinique, est la présence d'un variant de delta-globine ou d'une delta-thalassémie. L'absence totale de gamma-globine se traduit par l'absence de l'HbA2 mais reste sans conséquence pathologique.

L'HbA2 peut être faussement normale. Si le tableau hématologique et clinique correspond à une thalassémie mais sans répercussion sur le taux de A2 on doit penser à une association de plusieurs anomalies (bêtathalassémie associée à une carence martiale ou à une (delta-bêta-zéro thalassémie).

Quelles sont les causes d'une $\mathrm{Hb}$ A2 élevée ? La plus fréquente est une beta-thalassémie. En effet, en cas de bêta-thalassémie, le gène de deltaglobine est stimulé d'avantage par le LCR entraînant un taux plus élevé d'HbA2. Ce taux varie d'une mutation bêta-thalassémique à une autre et peut atteindre, dans certains cas, jusqu'à 15-17\% de l'hémoglobine totale. Une augmentation modérée du taux d'HbA2 est également décrite dans les anémies mégaloblastiques (carence en vit. B12) et à la suite de certains traitements antiviraux. La méthode classique de diagnostic d'une alphaou bêta-thalassémie est le dosage d'HbA2 effectué par des systèmes électrophorétiques (électrophorèse capillaire) ou chromatographiques (CEHPLC). Dans cette dernière méthode une cause d'erreur à connaître est l'élution de certains variants d'hémoglobine dans la fenêtre de 1'HbA2. Parmi ces variants on peut citer les $\mathrm{HbE}, \mathrm{Hb}$ Lepore, $\mathrm{Hb} \mathrm{D}$-Ouled Rabah, $\mathrm{Hb}$ D-Iran, $\mathrm{Hb}$ Fort de France, $\mathrm{Hb}$ Jeddah. En utilisant l'électrophorèse capillaire, on peut distinguer certains de ces variant de l'Hb A2. Parmi les hémoglobines issues de la fusion entre les gènes delta- et bêta-globine, on peut citer les Hbs Lepore (protéine de fusion delta-bêta) et les Hbs AntiLepore (protéine de fusion bêta-delta). Ces dernières donnent plusieurs hémoglobines en fonction de point de recombinaison (ex. : Hb P-Nilotic, $\mathrm{Hb}$ Miyada et $\mathrm{Hb}$ Hong Kong). L'Hémoglobine Hong Kong a la particularité de conserver le promoteur du gène bêta ?globine et d'avoir la partie codante du gène delta-globine. Nous rapportons ici le cas d'un sujet porteur d'une $\mathrm{Hb}$ Hong Kong chez qui la CE-HPLC a révélé un pic représentant $17 \%$ de l'Hb totale qui éluait dans la fenêtre de l'HbA2 et dont l'origine ethnique nous suggérait une $\mathrm{HbE}$ alors que les paramètres hématologiques étaient normaux. Des études phénotypiques et génétiques complémentaires ont permis le diagnostic d'une $\mathrm{Hb}$ Hong Kong à l'état hétérozygote.

Mots-clés : gène de fusion, Hémoglobine anti-Lepore, bêta-thalassémie et hémoglobine A2. 
EP369. PÉNÉTRANCE THÉRAPEUTIQUE DU GÉNOTYPE C282Y HOMOZYGOTE

E. Cadet (1), D. Capron (2), J.P. Ducroix (3), J.F. Cadranel (4), J. Rochette (1)

(I) Génétique UMR-INSERM U 925, Amiens, France; (2) Hépato-Gastroentérologie, CHU d'Amiens, France: (3) Médecine Interne, $\mathrm{CHU}$ d'Amiens, France ; (4) Hépato-Gastroentérologie, Hôpital Laënnec, Creil, France

Contact : jacques.rochette@u-picardie.fr

La Pénétrance d'un génotype est le nombre de sujets développant un phénotype spécifique rapporté au nombre de sujets porteurs du génotype correspondant. Elle est habituellement déterminée à partir d'un phénotype hérité sur le mode dominant et elle peut-être utilisée pour des calculs de risque dans certaines maladies génétiques. Elle présente donc un intérêt en santé publique. L'hémochromatose HFE est une surcharge en fer primaire d'origine génétique. Malgré son mode de transmission autosomique récessif, de très nombreuses études ont tenté d'établir la pénétrance du génotype morbide $\mathrm{C} 282 \mathrm{Y}$ homozygote. La plupart des études cherchent à établir deux valeurs de pénétrance : i) la pénétrance «clinique » basée sur la présence ou l'absence d'un certain nombre de symptômes plus ou moins spécifiques d'une surcharge en fer, ii) la pénétrance « biochimique » basée sur la valeur du coefficient de saturation de la transferrine (Sat \%) et/ou sur la valeur de la ferritine sérique (FS). Les valeurs de ces deux pénétrances (âge et sexe confondus) varient de $1 \%$ à $50 \%$ pour la pénétrance clinique et de $9 \%$ à $100 \%$ pour la pénétrance biochimique selon les auteurs. Cette dispersion des valeurs indique une hétérogénéité importante dans le choix des critères d'inclusion pour la définition du phénotype ainsi qu'un certain nombre de biais concernant le choix d'une population témoin. Afin de pallier ces difficultés, nous avons introduit la notion de "pénétrance thérapeutique $"$. Nous avons défini la pénétrance thérapeutique comme la proportion de sujets porteurs du génotype C282Y homozygote pour lesquels il est nécessaire de soustraire au moins 5 grammes de fer afin de normaliser la FS à une valeur $\leq 50$ microgrammes/L. La soustraction de 5 grammes de fer au moins, pour normaliser la FS est l'un des critères communément admis pour la définition phénotypique de l'hémochromatose. Entre 1997 et 2009 , nous avons suivi 808 sujets ( 477 hommes et 331 femmes) porteurs du génotype $\mathrm{C} 282 \mathrm{Y}$ homozygote. Au moment du diagnostic, les sujets ayant une FS $\geq 600$ microgrammes/L ont été soumis à un protocole de phlébotomies. Sur 331 femmes de plus de 45 ans, 39 seulement entraient dans la définition précédente. Pour les hommes de plus de 35 ans, 163 sujets sur 477 correspondaient au critère retenu. Dans notre série, la pénétrance thérapeutique du génotype C282Y homozygote est donc estimée à 0,14 chez la femme et à $0,34 \mathrm{chez}$ l'homme. Nous avons établi que la pénétrance thérapeutique représente environ la moitié de la pénétrance biochimique. La pénétrance thérapeutique n’introduit pas la confusion entre la prévalence de l'hémochromatose symptomatique aux différents stades de la maladie, relevant le plus souvent de l'expressivité variable, et la pénétrance clinique. Il n'est pas nécessaire d'utiliser une population témoin et elle est applicable à d'autres génotypes d'hémochromatose comprenant aussi le phénomène d'oligogénisme.

Mots-clés : pénétrance, mutation C282Y, HFE.

- 370 . DES MUTATIONS FAUX-SENS DANS LA SÉQUENCE CODANTE DE SOUS-UNITÉ L-FERRITINE : UNE NOUVELLE CAUSE GÉNÉTIQUE D'HYPERFERRITINÉMIE EN L'ABSENCE DE SURCHARGE EN FER

C. Kannengiesser (1, 3), A.M. Jouanolle (2), G. Hetet (1), A. Mosser (2), F. Muzeau (3), D. Henry (1), E. Bardou-Jacquet (2), M. Mornet (4), P. Brissot (5), Y. Deugnier (5), B. Grandchamp (1, 3), C. Beaumont (3)

(1) AP-HP, Service de Génétique et Biochimie hormonale, Hôpital Bichat Claude Bernard, Paris, France, Université Paris-Diderot, Paris, France ; (2) Laboratoire de Génétique Moléculaire, Hôpital Pontchaillou, Rennes, France ; (3) INSERM U773, Université Paris-Diderot, Paris, France ; (4) Service de Médecine Interne, Centre Hospitalier Jacques Caur, Bourges, France; (5) Service des Maladies du Foie et Centre de Référence des Surcharges Génétiques en Fer, Hôpital Pontchaillou, Rennes, France Contact : bernard.grandchamp@inserm.fr

La ferritine est une protéine composée de deux types de sous unités $\mathrm{H}$ et $\mathrm{L}$ dont le polymère permet le stockage intracellulaire du fer sous une forme non toxique. Chez les mammifêres une fraction de la sous-unité L synthétisée dans les hépatocytes est secrétée dans le plasma sous forme glycosylée tandis que la sous unité $\mathrm{H}$ n'est pas secrétée. Le rôle physiologique de la ferritine secrétée n'est pas connu et une hypothèse serait qu'elle puisse, au cours du développement embryonnaire, apporter du fer à certains types cellulaires sur lesquels un récepteur de la ferritine est exprimé Un taux élevé de ferritine sérique est fréquemment rencontré dans des situations cliniques variées et une fois écartées les causes habituelles d'hyperferritinémie (surcharge en fer, inflammation), de nombreux cas restent inexpliqués. Jusque récemment, la seule cause génétique connue d'hyperferritinémie sans surcharge en fer, souvent associée à une cataracte, était le syndrome héréditaire hyperferritinémie-cataracte (HHCS), transmis sur le mode autosomique dominant. Ce syndrome est lié à des mutations de I'IRE (iron responsive element) dans la région 5' non traduite de l'ARNm $\mathrm{L}$ ferritine, responsable de la régulation traductionnelle de cet ARNm. Nous avons étudié 93 proposants avec hyperferritinémie comprenant 26 cas appartenant à des familles ayant au moins deux cas d'hyperferritinémie inexpliquée, et 67 cas isolés. Dans les familles, nous avons également analysé 30 apparentés. L'hyperferritinémie était considérée comme inexpliquée en l'absence d'autre signe de surcharge en fer (saturation de la transferrine inférieure à $45 \%$ et/ou fer sérique inférieur à $25 \mu \mathrm{mol} / \mathrm{L}$ et/ou absence d'excès de fer tissulaire) et en l'absence d'inflammation (CRP normale). Nous avons exploré par séquençage des exons le gène codant pour la sous unité L ferritine (FTL) chez les cas index et 30 apparentés. Une mutation p.Thr30Ile hétérozygote a été trouvée chez 17 cas index de la cohorte et une autre mutation p.Gln26Leu chez deux cas index. Ces deux mutations sont situées dans l'hélice alpha située en position $\mathrm{N}$ terminale de la molécule et en augmentent l'hydrophobicité. La mutation p.Thr30Ile coségrège avec l'hyperferritinémie (transmission autosomique dominante) dans les 10 familles qui ont pu être étudiées. Aucun symptôme clinique spécifique n'a été trouvé associé à la présence de ces mutations. Tous les sujets porteurs de l'une de ces deux mutations présentent un pourcentage très élevé de la glycosylation de la ferritine sérique ( $>90 \%$, normales : $50-80 \%$ ) signant l'origine secrétée de la ferritine en excès. Ces mutations faux-sens de FTL représentent de nouvelles causes d'hyperferritinémie génétique, sans surcharge en fer et dont la reconnaissance peut éviter aux sujets concernés des investigations souvent nombreuses et parfois invasives. Ces mutations augmenteraient l'efficacité de la sécrétion de la L-Ferritine qui s'exerce en l'absence d'un peptide signal.

Mots-clés : hyperferritinémie, L-ferritine, ferritine secretée.

घP371. HÉTÉROZYGOTIE COMPOSITE ET PHÉNOTYPE D'HEMOCHROMATOSE JUVÉNILE TARDIVE : IDENTIFICATION DE NOUVEAUX GÉNOTYPES

E. Cadet (1), G. Le Gac (2), E. Letocart (2), I. Fajardy (3), M. Voicu (3), E. Verlet (4), R. Garidi (5), D. Capron (6), B. Heymann (7), C. Ferec (2), J. Rochette (1)

(1) Laboratoire de Génétique Moléculaire Médicale-INSERM UMR-925, CHU, Amiens, France; (2) Établissement Français du Sang-INSERM U613, Université de Bretagne Occidentale, Brest, France ; (3) Laboratoire de Biochimie, CHRU, Lille, France ; (4) Service de Médecine Interne, $\mathrm{CH}$, Dunkerque, France; (5) Service d'Hématologie Clinique, CHU, Amiens, France; (6) Service d'Hépato-Gastro- Entérologie, CHU, Amiens, France; (7) Centre de Gastro-Entérologie privé, Amiens, France Contact : estelle.cadet@u-picardie.fr

L'hémochromatose de type 2 ou hémochromatose juvénile est une forme sévère de surcharge en fer de transmission autosomique récessive. Il existe une hétérogénéité génétique avec 2 formes : $2 \mathrm{~A}$ et $2 \mathrm{~B}$ liées respectivement à des mutations dans les gènes codant l'hémojuvéline (HJV) et l'hepcidine (HAMP). Les formes les plus fréquentes sont dues à des mutations du gène HJV. L'hémochromatose $2 \mathrm{~A}$ est notamment illustrée par une hétérogénéité allélique importante et les hétérozygoties composites sont fréquentes. Nous décrivons quatre nouveaux génotypes d'hétérozygoties composites caractérisés par une présentation phénotypique tardive dans quatre familles différentes. Il s'agit respectivement des génotypes p.R92P/p.D172Y ; p.L194R/p.D149fsX245; p.R288Q/p.G320V et p.L194R/p.C347X. Les deux premiers génotypes ont été mis en évidence chez un seul membre de chaque famille. En revanche, les deux autres génotypes ont été trouvés respectivement chez trois frères et chez un frère et une sœur. Une variabilité importante est à noter dans l'âge des diagnostics: de 19 ans pour la patiente porteuse de l'hétérozygotie p.R92P/p.D172Y à 40 ans en moyenne pour ceux porteurs des mutations p.R288Q/p.G320V. Sur le plan clinique, une hyperferritinémie associée à une cardiomyopathie et à un hypogonadisme sont présents chez deux des sujets masculins porteurs d'une mutation non-sens. Par contre, la sceur dont le génotype est p.L194R/p.C347X présente une hyperferritinémie à $800 \mu \mathrm{gL}-1$ sans signe clinique. Les autres patients porteurs des hétérozygoties composites p.R92P/p.D172Y et p.R288Q/p.G320V présentent tous une surcharge en fer importante confirmée par IRM sans complication cardiaque ni endocrinienne. La recherche des mutations préalablement identifiées a pu être effectuée chez les parents des propositus dans trois des quatre familles (excepté celle du patient dont le génotype est p.L194R/p.D149fsX245). Dans tous les cas, chaque parent était porteur à l'état hétérozygote d'une des 
mutations retrouvées chez le probant. Aucun des parents hétérozygotes ne présentaient de surcharge en fer. Des tests in vitro basés sur la relation fonctionnelle décrite entre l'hémojuvéline membranaire et l'hepcidine ont été effectués. Ces derniers consistaient à mesurer et à comparer les activités transcriptionnelles du promoteur HAMP en réponse à l'HJV sauvage ou mutée, avec et sans BMPs (Bone Morphogenetic Proteins). Ces tests fonctionnels ont permis de révéler que toutes les protéines HJV mutées aux positions p.R92P, p.D172Y, p.L194R et p.C347X sont incapables de stimuler la transcription du gène HAMP. Ces résultats sont également obtenus avec la mutation la plus fréquemment décrite : p.G320V. Les hémojuvélines mutées, rapportées dans cette étude, sont donc incapables d'activer la synthèse de l'hepcidine via la voie de signalisation intracellulaire BMP/SMAD. Ces résultats illustrent de nouveau l'hétérogénéité allélique, génotypique et phénotypique de l'hémochromatose de type $2 \mathrm{~A}$.

Mots-clés: hémochromatose juvénile, hétérozygotie composite, hémojuvéline.

\section{IP372. DYNAMIQUE MOLÉCULAIRE EN SOLVANT EXPLICITE ET AIDE À L'INTERPRÉTATION DES CONSÉQUENCES DES MUTATIONS : APPLICATION AU VARIANT HFE P.R226G}

C. Cézard (1), G. Legac (2), C. Férec (2), J. Rochette (3)

(1) UMR-CNRS 6219, UPJV-Amiens, France ; (2) INSERM U 613, Brest, France ; (3) UMR-INSERM 925, UPJV-Amiens, France

Contact : jacques.rochette@u-picardie.fr

L'hémochromatose HFE est une maladie autosomique récessive liée à l'homozygotie p.C282Y. Quelques cas d'hétérozygotie composite ont été décrits, associant presque toujours la mutation p.C282Y à une mutation privée. Nous décrivons un nouveau génotype d'hétérozygotie composite p.C282Y/p.R226G, responsable d'une surcharge en fer (FS> 2000 microgrammes $/ \mathrm{ml}$, Csat $=75 \%$ ) chez un adulte de sexe masculin. Nous avons voulu savoir si la substitution R226G, vicinale du résidu cystéine 225 , pouvait générer un mécanisme susceptible de conduire à l'impossibilité de la création du pont disulfure 225 S-S 282 malgré la présence des 2 cystéines au sein du mutant. Cette hypothèse était basée sur un phénotype identique à celui qui est observé au cours de l'hémochromatose HFE due à l'homozygotie p.C282Y. Nous avons utilisé une méthode de dynamique moléculaire en solvant explicite, beaucoup plus proche de la réalité physiologique que les méthodes de modélisation par homologie ou en solvant implicite, déjà employées pour l'étude du variant p.Q283P. Nous avons effectué des simulations de 45 nanosecondes sur le domaine alpha3 de chacune de ces protéines (sauvage et mutée) en utilisant le champ de forces AMBER99SB développé au laboratoire. Les calculs ont été effectués à température et pression constantes, respectivement de $300^{\circ} \mathrm{K}$ et de 1 bar. Des études énergétiques et de conformation ont été réalisées afin d'étudier la stabilité du mutant. Nous montrons : i) que la structure secondaire de la protéine est conservée, en particulier la structure du feuillet beta dans lequel est localisée la mutation, n'est pas affectée, ii) nous notons pour le variant, des fluctuations de structures tertiaires, absentes dans la structure sauvage. En particulier, le résidu G226 induit un remaniement du groupement phényl du résidu Y64 alors qu'aucune interaction de ce type n'est décelable entre les résidus R226 et Y 64 de la protéine sauvage. Nous avons ensuite établi la distance entre les deux atomes de soufre impliqués dans le pont disulfure pour les deux protéines. Cette distance est de : $3,57 \AA+1-0,21$ pour la protéine sauvage, alors que pour le mutant cette distance est significativement plus grande : $4,25 \hat{A}+/-0,52$. Des résultats de calculs d'énergie libre de solvatation viennent renforcer l'hypothèse d'une déstabilisation importante de la conformation du mutant. En effet ; les temps nécessaires à la création de plusieurs ponts salins qui stabilisent normalement la structure tertiaire de la protéine sauvage sont significativement modifiés pour le variant. L'ensemble de ces résultats indique que la mutation $R 226 \mathrm{G}$ ne permet pas la création du pont disulfure $225 \mathrm{~S}-\mathrm{S} 282$ et introduit des modifications d'interaction charge-charge à distance de la substitution R226G Ce travail confirme l'intérêt de l'utilisation de l'outil de dynamique moléculaire en solvant explicite pour l'interprétation des conséquences des mutations lorsque des tests fonctionnels sont difficiles à développer.

Mots-clés : HFE, mutation, dynamique moléculaire.

IP373. IDENTIFICATION DE NOUVELLES MUTATIONS DANS LES GÈNES HFE, TFR2 ET SLC40A1 IMPLIQUÉS DANS LES FORMES RARES D'HÉMOCHROMATOSE HÉRÉDITAIRE

M. Voicu (1), E. Cadet (2), I. Fajardy (1), P. Thuillier (1), N. Roger (1), A. Tronc (1), E. Verlet (3), Ph. Mathurin (4), V. Canva (4), S. Ruet (1), V. Bailleul (1), J. Rochette (2)

(1) Laboratoire de Biochimie, CHRU Lille, France; (2) Laboratoire de Génétique Moléculaire Médicale, INSERM UMR-925, CHU Amiens,
Dunkerque, France; (4) Clinique des Maladies de l'Appareil Digestif et de la Nutrition, CHRU Lille, France

Contact : voicupm@yahoo.fr

Les hémochromatoses génétiques résultent de mutations dans plusieurs gènes codant des protéines impliquées dans le métabolisme du fer. Plusieurs formes de la maladie ont été décrites: l'hémochromatose classique de type 1 liée à des mutations dans le gène HFE, l'hémochromatose de type 2 ou juvénile due à des mutations dans les gènes codant soit l'hémojuvéline (type 2A) soit l'hepcidine (type 2B), l'hémochromatose de type 3 due à des mutations dans le gène codant le récepteur 2 de la transferrine (TfR2) et l'hémochromatose de type 4 liée à des mutations dans le gène SLC40Al codant la ferroportine. De nouvelles mutations ont été mises en évidence dans les gènes HFE, TfR2 et SLC40A1 chez des patients présentant une surcharge en fer. Nous avons mis en évidence dans le gène HFE une nouvelle hétérozygotie composite p.T117N/H63D chez un patient de 40 ans d'origine italienne, présentant une fatigue accentuée à l'effort, un coefficient de saturation de la transferrine de $70 \%$ et une ferritine de $342 \mathrm{ng} / \mathrm{mL}$. La mutation p.T117N est située dans l'exon 3 et n'est pas retrouvée dans la population générale, sur un échantillon test de 90 individus. Nous décrivons deux nouvelles mutations du gène TfR2: (1) la mutation p.Q672X (exon 16) à l'état homozygote provoque l'apparition prématurée d'un codon stop. Elle a été trouvée chez un homme de 40 ans présentant une forte surcharge en fer objectivée par la biologie et la biopsie hépatique. La surcharge décrite est très sévère et est associée à une fibrose septale mutilante. Par ailleurs, ce patient est hétérozygote HFE p.C282Y mais ne présente aucune mutation sur les autres gènes HJV, HAMP et SLC40A1 (2) une hétérozygotie composite associant une mutation connue p.I238M (exon 5) et une mutation nouvelle p.A45A a été retrouvée chez un patient de 49 ans d'origine africaine présentant une hyperferritinémie, une discrète surcharge hépatique en fer et aucune des 2 mutations p.C282Y et p.H63D du gène HFE. Enfin, nous rapportons une mutation nouvelle du gène SLC40A1 p.V161A à l'état hétérozygote (exon 5) chez un patient de 59 ans présentant un rhumatisme distal et une hyperferritinémie. Des études familiales et la recherche de ces anomalies dans la population générale sont en cours de réalisation afin de pouvoir affirmer ou non leur caractère pathogène. Des études fonctionnelles réalisées dans un second temps pourront confirmer les premiers résultats.

Mots-clés : hémochromatose HFE, TfR2, SLC40A1.

口P374. DÉLÉTIONS DU GÈNE HFE: CARACTÉRISATION MOLÉCULAIRE, PÉNÉTRANCE ET INTÉRÊT DIAGNOSTIQUE G. Le Gac (1), I. Gourlaouen (1), J.-M. Chen (1), S. Quemener (1), C. Ronsin (2), V. Géromel (2), C. Férec (1)

(1) Inserm U613, Établissement Français du Sang, Bretagne, Laboratoire de Génétique Moléculaire et d'Histocompatibilité du CHU de Brest, Université de Bretagne Occidentale, Brest, France; (2) LCL, service de biologie moléculaire, Ivry-sur-Seine, France

Contact : gerald.legac@univ-brest.fr

Introduction: Nous avons récemment décrit l'existence d'une délétion complète du gène HFE chez une femme de 47 ans. Bien que homozygote pour l'allèle délété, cette femme présentait un phénotype modéré de surcharge en fer [1]. La même délétion a été rapportée chez deux autres patients : une femme de 29 ans et un homme de 44 ans. Tous deux étaient homozygotes et tous deux présentaient des phénotypes sévères de surcharges en fer [2]. Les trois patients étaient d'origine sarde. Cela nous a conduit a réaliser une étude de population qui a confirmé l'existence d'un effet fondateur, et qui a également permis d'établir que la délétion du gène HFE était responsable d'une majorité de cas d'hémochromatose en Sardaigne [2]. Dans cette étude, nous rapportons l'existence d'un second type de délétion du gène HFE. Résultats : La délétion a été observée chez un homme de 60 ans, qui n'est pas d'origine sarde et chez qui une recherche de la mutation p.C282Y du gène HFE avait été prescrite en raison d'une élévation du coefficient de saturation de la transferrine $(95 \%)$. Une PCR spécifique de la délétion observée dans la population sarde a permis de révéler l'existence de points de cassures différents. Ce résultat a été confirmé par l'analyse de marqueurs du système HLA de classe I, mettants en évidence deux haplotypes, et par la mise en œuvre d'une technique de " marche sur le chromosome ». La caractérisation du second type de délétion du gène HFE n'est pas encore totalement achévée, mais il est déjà possible de souligner que les deux délétions ont été causées par des recombinaisons entre séquences Alu. Conclusions : Un parallèle peut être réalisé entre la variabilité phénotypique qui a été observée chez les patients homozygotes pour une délétion du gène HFE et celle qui est habituellement décrite pour le génotype p.C282Y/p.C282Y. Un parallèle peut également être réalisé avec les études fonctionnelles qui témoignent d'un rôle limité de la protéine HFE dans la régulation de la synthèse hépatique d'hepcidine, 
au regard de l'hémojuvéline et du récepteur à la transferrine de type 2. Les mutations du gène HFE apparaissent alors comme des facteurs nécessaires mais pas suffisants pour expliquer les phénotypes les plus sévères de surcharge en fer. Les séquences de type Alu apparaissent autour du gène HFE avec une fréquence plus importante $(17 \%)$ que celle que l'on peut observée en moyenne à l'échelle du génome $(5 \%)$. Une telle situation a été rapporté pour le gène SERPINC1 qui code l'antithrombine et dans lequel plusieurs délétions ont pu être identifiées [4]. Il ne serait donc pas surprenant que d'autres délétions puissent être découverte autour du gène HFE.

\section{Références}

1. Le Gac G., et al. Blood 2008.

2. Peluchi S., et al. Blood 2009.

3. Le Gac G., et al. Haematologica (in press).

4. Picard V., et al. Hum Genet 2009.

Mots-clés : hémochromatose, larges délétions.

\section{P375. MOI \\ COHORT}

M. Marduel (1, 2), M. Devillers (2), A. Marques (2), M. Abifadel (2), A. Carrié (3), A. Sassolas (4), D. Erlich (2), A. Munnich (1, 2), C. Junien (2), C. Boileau (2), M. Varret $(1,2)$, J.P. Rabès (2), The French ADH Research Network

(1) Université Paris-Descartes, Paris, France ; (2) INSERM U781, Paris, France ; (3) CHU Pitié Salpêtriere, Paris, France ; (4) CHU Antiquaille, Lyon, France

Contact : marie.marduel@inserm.fr

Autosomal Dominant Hypercholesterolemia (ADH) is characterized by isolated elevation of plasmatic LDL cholesterol associated with high risk of premature cardiovascular complications, that are the most important cause of morbidity and mortality in industrialized countries. Genetic factors involved in cholesterol homeostasis are multiple, but identification of genes linked to $\mathrm{ADH}$ have contributed to a better understanding of cholesterol metabolism. ADH was initially associated with mutations in 3 major genes: LDLR (encoding the low-density lipoprotein receptor), APOB (for the apolipoprotein B) and PCSK9 (for proprotein convertase subtilisin-kexin type 9). Over 1,000 mutations in the LDLR gene, 9 mutations in the APOB gene and 10 mutations in the PCSK9 gene have been implicated in ADH. Through the ADH French Research Network, we collected clinical, biological and molecular data from 392 French families. Mutations in the LDLR gene were identified in 286 families and are distributed as following : $46.9 \%$ missense, $17.5 \%$ nonsense, $10.3 \%$ splice, $12 \%$ frameshift, $1.7 \%$ in phase small insdel and $11.6 \%$ major rearrangements. Mutations were identified in 27 families for the APOB gene and in 8 families for the PCSK 9 gene. The 69 remaining families were named nonLDLR/nonAPOB/nonPCSK9 and are currently extended and analysed in order to identify other ADH genes. Thus, after the discovery of PCSK9 as the third ADH gene, our team has pursued the claim assertion that the disease is far more heterogeneous and that other $\mathrm{ADH}$ gene remain to be identified.

IP376. PCSK9 : DE LA RECHERCHE DE MUTATIONS AU DOSAGE SÉRIQUE DANS L'HYPERCHOLESTÉROLÉMIE FAMIIIALE

M. Abifadel $(1,2)$, J.P. Rabès $(1,3)$, A. Marquès (1), M. Marduèle (1), L. Tosolini (1), M. Devillers (1), M.E. Samson Bouma (1), A. Munnich (1), M. Varret (1), C. Boileau $(1,3)$

(1) Inserm U781, hôpital Necker-Enfants malades, Paris, France: (2) Faculté de pharmacie, université Saint-Joseph, Beyrouth, Liban ; (3) Laboratoire de biochimie et de génétique moléculaire, Hôpital Ambroise-Paré, Boulogne, France

Contact : marianne.abi-fadel@inserm.fr

L'hypercholestérolémie à transmission autosomique dominante touche un sujet sur 500 dans sa forme hétérozygote. Les gènes codant le récepteur des lipoprotéines de faible densité (LDLR) et son ligand l'apolipoprotéine B ont été longtemps les seuls incriminés dans cette pathologie. Nos travaux ont permis d'identifier en 2003, le troisième gène impliqué dans cette maladie : PCSK9, Proprotéine Convertase Subtilisine Kexine 9 (Abifadel et al. Nature Genetics, 2003). Plusieurs mutations hypercholestérolémiantes de PCSK9 (p.S127R, p.F216L, p.R218S...) entraînent un gain de fonction provoquant une diminution des taux de récepteurs des LDL en surface cellulaire. Tandis que d'autres variations sont hypocholestérolémiantes, notamment les mutations faux sens p.Y142X et p.C679X, retrouvées chez $2 \%$ des Afro-américains et associées à une forte réduction de l'incidence des accidents cardiovasculaires. PCSK9 est une enzyme circulante qui s'active par clivage auto-catalytique avant d'être sécrétée. Ses substrats et inhibiteurs ainsi que ses différents mécanismes d'action restent à élucider. Le dosage de PCSK9 dans le sang circulant est récemment possible grâce à des méthodes immunologiques (ELISA, Enzyme-linked

M/S hors sínie $n^{\circ} 1$, vol. 26, janvier 2010 immunosorbent assay). Nous avons ainsi pu mesurer la protéine PCSK9 pour la première fois dans le plasma de patients porteurs de 4 différentes mutations hypercholestérolémiantes du gène et de variations hypocholestérolémiantes. Les valeurs de PCSK9 obtenues fluctuent entre 50 et $680 \mathrm{ng} / \mathrm{ml}$, avec des valeurs moyennes de $310 \mathrm{ng} / \mathrm{ml}$. Bien que le nombre d'individus porteurs des mutations de PCSK9 soit minime, les valeurs obtenues pour 2 de ces mutations confirment les données obtenues dans les modèles cellulaires par des études de mutagénèse dirigée. Ceci contribue parallèlement aux études génétiques, aux modèles cellulaires et animaux que nous étudions, à une meilleure caractérisation du rôle de PCSK9 qui constitue actuellement une cible thérapeutique majeure dont l'inhibition pourrait agir en synergie avec les statines dans le traitement de l'hypercholestérolémie.

Mots-clés : hypercholestérolémie familiale, PCSK9, mutations.

IP377. NOVEL ZMPSTE24 (FACE1) MUTATIONS IN B-TYPE MANDIBULOACRAL DYSPLASIA AND RESTRICTIVE DERMOPATHY I. Agouti (1), V. Esteves-Vieira (1), P.S. Jouk (2), N. Elcioglu (3), P.C. Van den Akker (4), S.A. Lynch (5), C. Bennett (6), E. Hobson (7), S.A. Shalev (8), D. Mowat (9), N. Lévy (1, 10), A. De Sandre-Giovannoli $(1,10)$

(1) Laboratoire de Génétique Moléculaire, Département de Génétique Médicale, Hôpital la Timone Enfants, 264 Rue St Pierre, Marseille, France; (2) Génétique Médicale, Chu Michallon, Grenoble, France: (3) Department of Pediatric Genetics, Marmara University Hospital, Altunizade, Istanbul, Turkey; (4) Department of Clinical Genetics, University Medical Center Groningen, GRONINGEN, The Netherlands; (5) National Centre for Medical Genetics, Our Lady's Children's Hospital Crumlin, Dublin 12, Ireland; (6) Department of Clinical Genetics, Algernon Firth Building, Leeds General Infirmary, Great George Street, Leeds, UK ; (7) Department of Clinical Genetics, Chapel Allerton Hospital, Chapeltown Road, Leeds, UK ; (8) The Institute for Genetics, Ha'Emek Medical Center, Afula, Israel; (9) Sydney Children's Hospital Randwick. Sydney. Australia ; (10) INSERM UMR_S910, Faculté de Médecine de Marseille, 27 Bd Jean Moulin, Marseille, France

Contact : annachiara.desandre@ap-hm.fr

Homozygous mutations of ZMPSTE24, encoding a zinc metalloprotease specifically involved in the post-translational processing of prelamin A to mature lamin A, have been shown to cause two autosomal recessive disorders belonging to the phenotypic spectrum of systemic laminopathies : B-type Mandibuloacral Dysplasia (MAD-B) and Restrictive dermopathy (RD). Typically, ZMPSTE24 loss of function mutations lead to MAD-B, while null mutations lead to RD, respectively inducing partial or massive intracellular accumulation of toxic Prelamin A precursors. B-type MAD is characterized by mandibular hypoplasia, progressive lyses of the distal clavicles and phalanges, lipodystrophy, mottled cutaneous pigmentation and brittle and sparse hair. RD is an extremely severe genodermatosis, usually lethal in the perinatal period, characterized by a tight and rigid skin, multiple joint contractures, clavicular hypoplasia, generalized low bone density and pulmonary insufficiency generally leading to early death. We report eight novel homozygous or compound heterozygous ZMPSTE24 mutations and a novel LMNA heterozygous sequence variation observed in 7 families that were addressed to our laboratory for molecular genetic diagnosis. Among the ZMPSTE24 mutations, a three-nucleotide deletion was observed in two unrelated families affected with $\mathrm{B}$ type MAD; the seven other mutations, including a large deletion involving exons 9 and 10, four predicted missense mutations, one non sense and one donor splice site mutations, were observed in typical RD cases. One further case affected with RD carried the most frequent ZMPSTE24 mutation associated to this disease, i.e. a homozygous thymine insertion in exon 9. The mutations segregated following an autosomal recessive transmission pattern, as expected, in all the families. Functional analyses on lymphoblastoid or fibroblast cell lines could be carried out in some of the families, supporting the pathogenic effects of the mutations identified. Conversely, in one case of this series, the pathogenic effect of the mutation observed could not be ascertained, with obvious consequences for genetic counselling and eventual prenatal diagnosis issues.

Mots-clés : laminopathy, ZMPSTE24, restrictive dermopathy.

EP378. RECHERCHE DE LA MUTATION RÉCURRENTE P.GLY608GLY CHEZ TROIS PATIENTS MAROCAINS ATTEINTS DU SYNDROME D'HUTCHINSON-GILFORD (PROGERIA)

A. Lamzouri (1), Y. Doubaj (1), I. Ratbi (1, 2), S. Chafai Elalaoui (1), F.Z. Laarabi (1), A. Sefiani $(1,2)$

(I) Département de Génétique Médicale, Institut National d'Hygiène, Rabat, Maroc; (2) Centre de Génomique Humaine, Faculté de Médecine et de Pharmacie de Rabat, Université Mohammed V Souissi, Rabat, Maroc Contact : lamzouriafaf@hotmail.com 
Le syndrome d'Hutchinson-Gilford ou Progèria est une maladie génétique extrêmement rare dont la prévalence est estimée à 1 sur 8 millions. Elle est caractérisée par un vieillissement prématuré et une atteinte multisystémique sans retard mental, entrainnant un décès précoce à la $1^{\text {Te }}$ ou $2^{\mathrm{C}}$ décade de vie. Son mode de transmission est autosomique dominant, avec une pénétrance complète. Le gène impliqué est le LMNA localisé en 1q21.2 codant pour les protéines lamines A et C. Dans plus de $80 \%$ des cas, il s'agit d'une substitution hétérozygote d'une seule paire de base, apparue de novo au niveau de l'exon 11 ; c. $1824 \mathrm{C}>\mathrm{T}$; p.Gly608Gly, qui provoque la création d'un site donneur d'épissage, aboutissant à la formation d'une protéine lamine A tronquée. Celle-ci s'accumule sous forme d'un précurseur (prélamine A) dans les noyaux des cellules où elle exerce des effets délétères. Nous rapportons dans ce travail les observations de trois patients marocains, deux filles et un garçon âgés de 5 à 12 ans, suivis en consultation de génétique pour un tableau classique de Progèria. Un seul de nos patients, avaient la mutation récurrente c.1824C $>T$; p.Gly608Gly du gène LMNA à l'état hétérozygote. Le séquençage de la totalité de ce gène est en cours pour identifier la mutation causale chez les deux autres patients.

Mots-clés : Hutchinson, Gilford, LMNA, mutation.

\section{IP379. NOTRE EXPÉRIENCE DANS LE DIAGNOSTIC DES MALADIES LYSOSOMALES}

B. Imessaoudene, A. Zellaguil, D. Ghris, A. Boughalem, M.T. Hassen, A. LebdjiriI, I. Amara, A. Berhoune

Laboratoire de Biochimie, CHU Mustapha, Alger, Algérie

Contact : imessaoudene@hotmail.com

Les maladies lysosomales recouvrent l'ensemble des affections métaboliques caractérisées par une accumulation pathologique de substance de réserve. Elles comprennent une cinquantaine de maladies présentant une symptomatologie avec des répercussions neurologiques graves. La structure familiale dans notre population est fortement imprégnée de consanguinité. Ceci est soutenu par le nombre important de maladies dont le diagnostic phénotypique à été posé dans notre laboratoire. Ce diagnostic phénotypique repose essentiellement sur la mise en évidence des métabolites urinaires et sanguins ainsi que sur les activités enzymatiques. Depuis plusieures années les malades nous sont adressés par les services de pédiatrie et de neurologie de tout le pays. Dans notre bilan sur les trois dernières années nous retrouvons par ordre d'importance les maladies suivantes: Gauchers $=40$ cas Leucodystrophie métachromatique $=16$ cas Landing $=14$ cas Mucopolysaccharidoses $=40$ cas Le diagnostic génotypique à été réalisé seulement pour quelques Gauchers. La demande était justifiée par la mise en œuvre d'un traitement substitutif. Le conseil génétique serait une finalité importante pour fournir à ces familles un indice permettant de prévenir la survenue d'autres enfants atteints.

Mots-clés : lysosome, maladies génétiques, enzymothérapie.

\section{IP380. ANALYSE MUTATIONNELLE DU GENE HGNSAT CHEZ 2 FAMILLES MAROCAINES ATTEINTES DU SYNDROME DE SANFILIPPO TYPE C}

S. Chafai Elalaoui (1), I. Cherkaoui Jaouad (1), I. Canals (2), D. Grinberg (2), L. Vilageliu (2), A. Sefiani $(1,3)$

(1) Département de génétique médicale, Institut National d'Hygiène, Rabat, Maroc: (2) Centre de génomique humaine, Faculté de médecine et de pharmacie de Rabat, Université Mohammed V Souissi, Rabat, Maroc; (3) Centre de génomique humaine, Faculté de médecine et de pharmacie de Rabat, Université Mohammed V Souissi, Rabat, Maroc Contact : sihamgen@yahoo.fr

La mucopolysaccharidose de type III ou syndrome de Sanfilippo (SS) est une maladie de surcharge lysosomale, du groupe des mucopolysaccharidoses, de transmission autosomique récessive. Elle se caractérise par un retard d'apparition des traits dysmorphiques, une détérioration neuropsychique, et le décès survient vers l'âge de 20 ans. Elle est due à la présence d'héparane sulfate non dégradé en raison du déficit de l'une des quatre enzymes nécessaires à son catabolisme, définissant ainsi les types A, B, C et D. Le SS type C, est dû au déficit en Acétyl CoA : alpha-glucosaminide-acétyltransférase, codée par le gène HGNSAT récemment cloné et identifié. Plusieurs mutations ont été rapportées dans ce gène, dont la mutation récurrente c. $[318+1 \mathrm{G}>\mathrm{A} ; 794 \mathrm{C}>\mathrm{A}]$, rapportée chez deux patients marocains et un patient espagnol. Nous avons recherché chez deux familles marocaines consanguines, non apparentés ayant le SS type C cette mutation récurrente. Pour l'une des familles, le SS était associé à une puberté précoce chez les deux sæurs atteintes dans cette famille. Nous avons commencé par la recherche de la mutation récurrente c. $[318+1 \mathrm{G}>\mathrm{A} ; 794 \mathrm{C}>\mathrm{A}]$, qui n'a été trouvée que chez une famille. Tandis été trouvée, dont les études fonctionnelles ont montré que l'enzyme ayant cette nouvelle mutation n'a aucune activité. Nous nous sommes intéressés à la causalité de la substitution c.794C $>$ A (P265Q) dans la mutation complexe c. $[318+1 \mathrm{G}>\mathrm{A} ; 794 \mathrm{C}>\mathrm{A}]$. Nous avons recherché la substitution c. $794 \mathrm{C}>\mathrm{A}$ chez 61 témoins marocains, parmi lesquels 2 d'entre eux l'avaient (un homozygote et un hétérozogote), ce qui correspond à 3 chromosomes sur 122. Par ailleurs des études de fonction ont montré que l'enzyme ayant cette substitution présente une activité de $96 \%$ par rapport à l'enzyme normale, et que l'enzyme ayant la mutation complexe a une activité très diminuée. Ceci nous permet de conclure que la mutation complexe c. $[318+1 \mathrm{G}>\mathrm{A} ; 794 \mathrm{C}>\mathrm{A}]$ serait au fait la mutation d'épissage c. $318+1 \mathrm{G}>\mathrm{A}$. Ces résultats nous permette de proposer la recherche de cette mutation récurrente en première intention chez les patients marocains ayant le syndrome de Sanfillippo type C.

Mots-clés : gène HGNSAT, nouvelle mutation, mutation récurrente.

IP381. IDENTIFICATION ET CARACTÉRISATION D'UNE DÉLÉTION INTRAGÉNIQUE HOMOZYGOTE DU GÈNE ALPHAN-ACÉTYLGLUCOSAMINIDASE CHEZ UN PATIENT ATTEINT D'UNE MALADIE DE SANFILIPPO DE TYPE B (MUCOPOLYSACCHARIDOSE IIIB)

I. Maystadt (1), A. Destrée (1), P. Vannuffel (1), C. Verellen-Dumoulin (1), K.J. Champion (2), T. Wood (2), M.J. Basehore (2)

(1) Institut de Pathologie et de Génétique, Gosselies, Belgique ; (2) Greenwood Genetic Center, Greenwood, South Carolina, USA

Contact : isabelle.maystadt@ipg.be

La maladie de Sanfilippo de type B (mucopolysaccharidose IIIB) est une maladie de transmission autosomique récessive, résultant d'une accumulation de glycosaminoglycans dans les lysosomes suite à un déficit en alpha- $\mathrm{N}$-acétylglucosaminidase, une enzyme lysosomale codée par le gène NAGLU. La maladie est évolutive et se manifeste par un retard de développement psychomoteur apparaissant le plus souvent entre l'âge de un et quatre ans, suivi de troubles du comportement de type agressivité et agitation, et d'une détérioration progressive des fonctions neurologiques tant sur le plan moteur que cognitif. Plus de 100 mutations différentes du gène NAGLU ont déjà été répertoriées chez les patients atteints de la maladie de Sanfilippo, mais aucune délétion intragénique de grande taille n'a été rapportée à ce jour. Chez une patiente de sept ans, issue de parents consanguins et présentant un phénotype classique de maladie de Sanfilippo avec déficit prouvé en alpha- $\mathrm{N}$-acétylglucosaminidase, nous décrivons ici pour la première fois une délétion intragénique de 1146 paires de bases, emportant les exons 3 et 4 du gène NAGLU. Cette délétion provient vraisemblablement d'une recombinaison homologue non-allélique entre deux séquences Alu (séquence Alu-Y dans l'intron 2 et séquence Alu-Sx dans l'intron 4). Les séquences Alu ont déjà été incriminées dans la pathogenèse de plusieurs maladies génétiques humaines (hypercholestérolémie, maladie de Duchenne, maladie de Tay-Sachs,...). Cette observation confirme donc le rôle des séquences Alu dans la survenue de délétions intragéniques. L'existence de délétions intragéniques dans le gène NAGLU est une information capitale pour le conseil génétique. Il convient en effet de différencier les patients homozygotes des patients hétérozygotes composites pour une délétion intragénique et une mutation ponctuelle du gène NAGLU. En cas de découverte d'une mutation apparemment homozygote, il est donc indispensable d'en tester l'origine parentale et de compléter si nécessaire le séquençage du gène par des méthodes de dosage génique.

Mots-clés : mucopolysaccharidose, deletion, séquence Alu.

\section{IP382. NOUVELLE MALADIE DE SURCHARGE RESSEMBLANT} AU SYNDROME DE MORQUIO CHEZ DEUX FRÈRES

L. Perrin (1), O. Fenneteau (2), C. Baumann (1), M. Gérard (1), K. Mazda (3), A. Verloes (1)

(1) Génétique clinique, Hôpital Robert-Debré, Paris, France ; (2) Laboratoire d'hématologie, Hôpital Robert-Debré, Paris, France; (3) Chirurgie orthopédique, Hôpital Robert-Debré, Paris, France

Contact : laurence.perrin@rdb.aphp.fr

Nous rapportons le cas de deux frères issus de parents d'origine antillaise non apparentés présentant une maladie de surcharge non identifiable. Les deux enfants ont la même histoire clinique : grossesse et la naissance normale, impression de micromélie dès les premiers mois de vie, puis retard statural progressif et déformations squelettiques évoquant une maladie de Morquio. On observe une petite taille (-4DS), un tronc court, un thorax bombé avec un pectus carinatum, un cou court, une scoliose sévère, une micromélie rhizomélique, des pieds petits et adductus ainsi qu'une hyperlaxicité des petites articulations et un genu valgum. Il n'y a pas d'hépatosplénomégalie ni de dysmorphie faciale. Le périmètre crânien est normal 
et l'examen neurologique sans particularité. La marche a été acquise à un âge normal mais les deux enfants ont présenté des difficultés d'apprentissage. La scoliose sévère et évolutive a nécessité une correction chirurgicale chez les deux patients à 4 et 6 ans. Les radiographies osseuses mettent en évidence une platyspondylie généralisée avec une déformation en rostre des corps vertébraux et une hypoplasie de l'odontoïde. Il existe également une dysplasie épiphysométaphysaire. L'examen ophtalmologique est sans particularité (pas de dépôts cornéens). Malgré des screenings urinaires itératifs, des traces de dermatane sulfate ont été retrouvées une seul fois, chez l'un des deux patients. Au frottis sanguin, on n'observe pas de lymphocytes vacuolés, alors que les éosinophiles présentent des granulations anormales de teintes variées et qu'il existe un renforcement des granulations azurophiles des polynucléaires (anomalie d'Alder). La biopsie de peau ne montre pas de signes de surcharge. Les dosages enzymatiques ont permis d'exclure une maladie de Morquio ( $\mathrm{N}$-acetylgalactosamine-6-sulfatase and betaD-galactosidase), une maladie de Maroteaux-Lamy (arylsulfatase B), une mucolipidose (hydrolases acides), une mannosidose (beta mannosidase), une maladie de Hurler (alpha-L-iduronidase). En conclusion : ces deux enfants présentent une maladie de surcharge non classifiable, ressemblant sur le plan clinique et radiologique à la maladie de Morquio. Le déficit enzymatique causal n'est pas identifié à ce jour.

Mots-clés : morquio, maladie de surcharge.

IP383. CARACTÉRISATION MOLÉCULAIRE DE LA MALADIE DE SANFILIPPO CHEZ DES PATIENTS TUNISIENS

S. Ouesleti (1, 4), H. Ben Turkia (2), H. Dranguet (3), P. Saugier-veber (3), A. Miled (1), N. Miladi (4), S. Bekri (3)

(I) Laboratoire de Biochimie de l'Hôpital Farhat Hached, Sousse, Tunisie ; (2) Service de pédiatrie de la Rabta, Tunis, Tunisie ; (3) Fédération de Génétique-CHU de Rouen, France; (4) Unité de recherche des Maladies Neurologiques de L'Enfant, Faculté de Médecine de Tunis, Tunisie

Contact : Soumeya.Bekri@chu-rouen.fr

La mucopolysaccharidose de type III (MPS III) ou maladie de Sanfilippo est une maladie lysosomale caractérisée par le déficit d'une des 4 enzymes intervenant dans le catabolisme de l'héparane sulfate : héparane sulfamidase-type IIIA, alpha-N-acétylglucosaminidase-type IIIB, acétyl$\mathrm{CoA}$ :alpha-glucosaminide- $\mathrm{N}$-acétyltransférase- type IIIC, et $\mathrm{N}$-acétylglucosamine-6-sulfate sulfatase-type IIID. Les manifestations cliniques sont dominées par un retard mental et une atteinte du système nerveux central. Cette pathologie est sous-diagnostiquée car l'atteinte viscérale est généralement mineure et la dysmorphie faciale reste modérée. Le pronostic sévère est lié à la détérioration neurologique. Cette étude a porté sur la caractérisation clinique et moléculaire de 9 familles tunisiennes ayant une maladie de Sanfilippo ( 10 cas index et 22 apparentés). Une recherche de mutations par séquençage direct des exons, jonctions intron-exon et de la région promotrice a été effectuée pour les gènes SGSH (MPS IIIA), NAGLU (MPS IIIB) et HGSNAT (MPS IIIC) Le gène SGSH a été étudié dans 4 familles MPS IIIA, 6 mutations ont été identifiées dont 4 non répertoriées. Le génotypage de NAGLU dans 3 familles MPS IIIB a conduit à la caractérisation de 2 mutations non décrites précédemment. Le séquençage de HGSNAT dans 2 familles MPS IIIC a mis en évidence la présence de 2 nouvelles mutations. Le taux de consanguinité très élevé en Tunisie laisserait supposer une prévalence plus élevée dans cette population. La caractérisation moléculaire de la maladie de Sanfilippo est un préalable à l'optimisation de nouvelles perspectives thérapeutiques de type molécules chaperonnes et permet de proposer un diagnostic anténatal le cas échéant.

EP384. LA MALADIE DE GAUCHER EN TUNISIE À PROPOS DE HUIT CAS ADULTES

F. Ben Rhouma (1, 2), H. Ben Turkia (3), W. Cherif (1, 3), N. Tebib (3), H. Azzouz (3), S. Mseddi (4), M.F. Ben Dridi (3), S. Abdelhak (1)

(1) Unité SP 26/04 d'Exploration Moléculaire des Maladies Orphelines d'Origine Génétique, Institut Pasteur de Tunis, Tunisie ; (2) Unité 05/UR/ 08-02 des maladies neurologiques de l'enfant, Faculté de Medecine de Tunis, Tunisie; (3) Unité 25/04 de Dépistage et de Prise en charge des Maladies Héréditaires du Métabolisme, Service de Pédiatrie du CHU la Rabta Tunis, Tunisie ; (4) Service et Laboratoire d'Hématologie du CHU Hédi Chaker de Sfax, Tunisie

Contact : benrhouma_faten@yahoo.fr

La maladie de Gaucher est la maladie de surcharge lysosomale la plus fréquente. De transmission autosomique récessive, elle est due à un déficit enzymatique en beta-glucocérébrosidase qui entraîne l'accumulation pathologique du substrat (le glucosylcéramide) dans les lysosomes des macrophages. Cette surcharge est responsable des manifestations cliniques de l'affection. Elle est caractérisée par une hétérogénéité phénotypique considérable. Elle est subdivisée en trois types (Type 1 : MIM 230800; Type 2 : MIM 230900 ; Type 3 : MIM 231000) classés selon l'absence ou la présence de manifestations neurologiques. De plus, elle présente une très grande hétérogénéité génétique. Plus de 200 mutations touchant le gène GBA codant pour cette enzyme ont été identifiées. Certaines mutations surviennent par des mécanismes de recombinaison entre le gène GBA et son pseudogène psGBA. La proximité de ces deux derniers complique l'analyse génotypique et interfère dans le dépistage de quelques mutations dans ce gène. Dans le présent travail, nous avons cherché trois mutations récurrentes (N370S, L444P et RecNcil) chez huit patients adultes tunisiens atteints de la maladie de Gaucher. Le dépistage de ces mutations a été réalisé par la méthode de PCR/RFLP puis confirmé par séquençage. Les résultats ont révélé que 5 patients sont homozygotes N370S/N370S ; un seul patient hétérozygote $\mathrm{N} 370 \mathrm{~S} / \mathrm{RecNciI}$ et deux patients hétérozygotes N370S/L444P. À travers notre étude nous avons remarqué une homogénéité du spectre mutationnel de la maladie de Gaucher chez les patients adultes dans la population Tunisienne ; ce qui fournit un outil de diagnostic moléculaire très important. De plus, nous avons trouvé qu'un des patients explorés a développé la maladie de Parkinson. Sachant qu'il est le seul qui porte la mutation RecNcil, une hypothèse peut être évoquée sur l'association de cette mutation avec l'apparition de la maladie de Parkinson chez les patients adultes tunisiens atteints de la maladie de Gaucher.

Mots-clés : maladie de Gaucher, gène GBA, beta-glucocérébrosidase.

IP385. MALADIE DE SAN FILIPPO : CAS CLINIQUE D'UNE FAMILLE MAROCAINE

H. Dehbi (1), H. Hjiej (2), S. Nassereddine (1), S. Nadifi (1), G. Benjalloun (2) (1) Laboratoire de Génétique Médicale, Faculté de Médecine et de Pharmacie de Casablanca, Maroc: (2) Unité de pédopsychiatrie, hôpital des enfants, CHU Ibn Rochd, Casablanca, Maroc

Contact : hind500@yahoo.fr

La maladie de San Filippo ou la mucopolysaccharidose (MPS) de type III est une maladie de surcharge lysosomale caractérisée par un défaut de dégradation de l'héparane sulfate(HS) suite à un déficit enzymatique. Sa prévalence est variable car elle est souvent sous-diagnostiquée, mais elle reste plus fréquente en Europe qu'en Afrique. Cette pathologie autosomale récessive est caractérisée par une dégradation intellectuelle sévère et rapide. Nous rapportons dans ce travail l'observation clinique d'une famille marocaine atteinte de cette encéphalopathie métabolique. Il s'agit de deux frères âgés respectivement de 5 ans et 16 ans, issus de parents consanguins, et qui présentent un retard global de développement. L'examen clinique retrouve chez les deux garçons une dysmorphie faciale légère avec déformation des membres inférieurs en genu valgum et pieds bots varus, l'évaluation pédopsychiatrique retrouve un retard mental avec trouble de la compréhension. Le benjamin présente en plus, un retard de langage et des acquisitions psychomotrices avec troubles du comportement et instabilité, sans agressivité ni crises épileptiques associées. Par contre, le frère aîné présente une forme plus atténué, il est autonome et scolarisé dans une école spécialisée, et sa symptomatologie se limite à un bégaiement. La pathologie a été confirmée par le dosage élevé de l'HS dans les urines. Le dosage dans les leucocytes des quatre enzymes impliquées est en cours de réalisation, il permettra de préciser le sous type et d'orienter le conseil génétique. La recherche des mutations ne pourra être réalisée qu'après la détermination du sous type. L'évolution a été marquée par une amélioration nettement positive du langage et du comportement après une année de suivi médical, de rééducations intensives, et de prise en charge en psychothérapique.

Mots-clés : mucopolysaccharidose type III, héparane sulfate, déficience intellectuelle.

DP386. CARACTÉRISATION MOLÉCULAIRE DES GLYCOGÉNOSES HÉPATIQUES PAR DÉFICIT EN PHOSPHORYLASE (TYPE VI) ET PHOSPHORYLASE KINASE (TYPE VIII)

A. Spraul (1), D. Dobbelaere (2), D. Habes (3), V. Valayannopoulos (4), P. Labrune (5), O. Bernard (3), E. Jacquemin (3), C. Baussan (1)

(1) Laboratoire de Biochimie, CHU Bicêtre, APHP, France; (2) Centre de Référence des Maladies Héréditaires du Métabolisme, CHU Lille, France; (3) Service d'Hépatologie Pédiatrique et Centre de Référence de Maladies Rares, CHU Bicêtre, APHP, France; (4) Centre de Référence des Maladies Métaboliques, CHU Necker, APHP, France; (5) Centre de Référence des Maladies Héréditaires du Métabolisme Hépatique, CHU Antoine-Béclère, APHP, France

Contact : anne.spraul@bct.aphp.fr

Les glycogénoses de type VI (GSDVI) et de type VIII (GSDVIII) sont la conséquence d'un dysfonctionnement du système de la phosphorylase 
(PHase) hépatique qui catalyse la première étape de la dégradation du glycogène. Ces maladies génétiques sont liées soit à un déficit en PHase hépatique (gène PYGL), soit à un déficit en phosphorylase kinase (PHK), complexe multienzymatique activant la PHase et formé par l'association de plusieurs sous-unités, dont les sous-unités alpha et gamma respectivement codées par les gènes PHKA2 et PHKG2. Les déficits en PYGL et PHKG2 sont transmis selon un mode autosomique récessif, le déficit en PHKA2 est lié au chromosome X (XLG). L'orientation initiale est clinicobiologique : hépatomégalie, cytolyse, retard de croissance, bonne tolérance au jeûne. Le diagnostic enzymatique direct peut être effectué sur cellules sanguines mais n'est pas toujours informatif du fait d'une activité résiduelle de la PHase ou d'une expression incomplète du déficit en PHK « variants XLG2 ». Le diagnostic nécessite alors de faire une biopsie hépatique pour mesurer l'activité enzymatique hépatique. La recherche de mutation représente maintenant une alternative pour confirmer le diagnostic et éviter un geste invasif. Cette étude rapporte la caractérisation moléculaire de 20 patients atteints de GSDVI ou GSDVIII après orientation du déficit enzymatique sur cellules sanguines. Patients et Méthodes : Le séquençage du gène PYGL a été réalisé chez les enfants suspects de GSDVI. Chez les garçons atteints de GSDVIII modérée, l'étude du gène PHKA2 a été réalisé d'emblée. Par contre, chez les filles et les garçons avec un phénotype sévère, le gène PHKG2 a été séquencé en première intention. Résultats : Un déficit enzymatique en PHase, plus ou moins prononcé, sans défaut d'activation de la PHase, a été retrouvé chez 8 patients. L'étude moléculaire du gène PYGL a permis de confirmer le diagnostic de GSDVI et d'identifier 10 nouvelles mutations et 2 décrites. Ces mutations étaient homozygotes ou hétérozygotes composites. Chez les 12 autres patients, la diminution de l'activité PHase était associée à un défaut d'activation et à une diminution de l'activité PHK sur cellules sanguines, excepté chez 3 patients présentant uniquement un défaut d'activation * variants XLG2 . L'étude du gène PHKA2 a révélé des mutations hémizygotes chez 10 garçons avec des mutations faux sens chez les 3 « variants XLG2 ", L'analyse du gène PHKG2, réalisée chez les 2 autres enfants a retrouvé une délétion homozygote chez le garçon mais aucune mutation chez la fille. Conclusion : La sélection des patients atteints de GSDVI ou GSDVIII par l'étude enzymatique sur cellules sanguines permet d'orienter le choix du gène à séquencer. L'étude moléculaire a confirmé le diagnostic dans la quasi totalité des cas et suggère que l'on peut surseoire à la biopsie hépatique si l'étude enzymatique sur cellules sanguines n'est pas informative.

Mots-clés : PYGL, PHKA2, PHKG2.

\section{EP387. DESCRIPTION MOLÉCULAIRE DES MUTATIONS DANS PGM1 RESPONSABLES DE LA GLYCOGÉNOSE DE TYPE XIV} L. Capel (1), T. Stojkovic (2), M. Piraud (3), F. Parisot (1), P. Laforêt (2), F. Petit (1)

(I) Service de biochimie et hormonologie, unité de génétique, Hôpital Antoine-Béclère (APHP), Clamart, France; (2) Service de neurologie et maladies musculaires, Institut de myologie, Groupe hospitalier Pitié-Salpétrière (APHP), Paris, France: (3) Service des maladies métaboliques, Hospices Civils de Lyon, Lyon, France

Contact : francois.petit@abc.aphp.fr

Introduction. Les glycogénoses sont des pathologies rares touchant les enzymes du métabolisme du glycogène et, par extension, des enzymes du métabolisme des produits de dégradation du glycogène. Ces affections ont des répercussions hépatiques et/ou musculaires pouvant être à l'origine d'une maladie métabolique potentiellement létale et/ou d'une maladie musculaire pouvant être responsable de myopathie. Matériels et méthodes. Nous rapportons ici le cas d'un patient présentant un déficit musculaire modéré se caractérisant principalement par une intolérance à l'effort (crampes musculaires) avec des signes de rhabdomyolyse. L'étude biochimique de la cascade enzymatique de la glycogénolyse a mis en évidence un déficit en activité phosphoglucomutase. Le but de ce travail était de caractériser sur le plan moléculaire le déficit en phosphoglucomutase. Il existe 4 gènes codant une protéine à activité phosphoglucomutase mais une analyse des profils d'expression nous a orienté vers l'étude du gène de la phosphoglucomutase de type 1 (PGM1). Les ADN du patient et de ses parents ont été extraits à partir des leucocytes circulants. Les 12 exons du gène PGM1 (exo 1A de la protéine PGM1 commune et exon 1B de la protéine PGMI musculaire) ont été amplifiés, séquencés et comparés à la séquence de référence (Genebank accession BC019920). Résultats. L'analyse de la séquence du gène PGMI chez notre patient a permis de mettre en évidence deux anomalies de séquence en trans : - la mutation c.343A>G d'origine paternelle responsable de la modification de séquence peptidique p.Thr115Ala dans l'exon 2 (acide aminé hautement conservé), - la mutation c.1145-1G $>\mathrm{C}$ d'origine maternelle dans le site donneur d'épissage de l'intron 7-8. La mutation c. $343 \mathrm{~A}>\mathrm{G}$ a été recherchée dans une population de 64 témoins adultes également été retrouvé chez le patient (rs855314). Discussion - conclusion. Il s'agit de la première description d'une anomalie moléculaire à l'origine d'un déficit en activité phosphoglucomutase responsable d'une atteinte musculaire modérée. Il a été proposé de classer ce déficit comme la glycogénose de type XIV. Il est probable que d'autres anomalies seront décrites dans ce gène en étudiant des patients présentant des signes cliniques similaires et jusque là peu ou pas explorés sur le plan moléculaire.

Mots-clés : phosphoglucomutase, glycogénose

-P388. FACTEURS GÉNÉTIQUES DE L'HYPERBILIRUBINÉMIE NON-CONJUGUÉE EN PÉRIODE NÉONATALE

E. Brisselet (1), M. Hébert (1), L. Capel (1), F. Parisot (1), C. Poüs (1), P. Labrune (2), F. Petit (1)

(1) Service de biochimie et hormonologie, unité de génétique, Hôpital Antoine-Béclère (APHP), Clamart, France; (2) Service de pédiatrie, Centre de référence maladies héréditaires du métabolisme hépatique, Université Paris Sud, INSERM U 948, Hôpital Antoine-Béclère (APHP), Clamart, France

Contact : francois.petit@abc.aphp.fr

L'hyperbilirubinémie non-conjuguée est une situation fréquente en période néonatale, en particulier en cas de prématurité. Souvent bénigne, I'hyperbilirubinémie peut cependant, dans certains cas, atteindre des concentrations dépassant les $250 \mu \mathrm{mol} / \mathrm{L}$ pouvant être à l'origine d'une atteinte neurologique et nécessitant une prise en charge rapide et efficace alliant photothérapie et parfois exsanguinotransfusion. En dehors des cas d'hyper-hémolyse, les causes sont souvent mal identifiées. Le but de cette étude était de déterminer si la démarche biologique consistant en l'analyse du promoteur du gène UGT1A1 était nécessaire et suffisante pour répondre à la demande clinique de diagnostic étiologique de l'hyperbilirubinémie en période néonatale. 103 nouveau-nés à terme ayant présenté une hyperbilirubinémie non-conjuguée avec des concentrations en bilirubine comprises entre 85 et $727 \mu \mathrm{mol} / \mathrm{L}$ ont été inclus. Les données moléculaires obtenues ont été comparées avec celles obtenues chez 64 témoins sains adultes. Nous avons étudié les polymorphismes et séquences suivantes: le polymorphisme A(TA)6TAA/ A(TA)7TAA du promoteur du gène UGT1A1 (TATAbox), la séquence des 5 exons du gène UGT1A1 (exons $1,2,3,4$ et 5 ), le polymorphisme c.-3275T>G de la séquence de liaison du phénobarbital en amont du gène UGTIA1 (PBREM), deux polymorphismes c.388A $>\mathrm{G}$ et c.521T $>\mathrm{C}$ dans le gène du canal OATP2 (OATP2 ex4 et OATP2 ex5), le polymorphisme (GT)n de la séquence promotrice du gène de l'hème-oxygénase 1 (HOX1). Concernant les deux polymorphismes du canal OATP2 et le polymorphisme de la séquence promotrice du gène $\mathrm{HOX} 1$, aucune différence significative n'a été mise en évidence entre les deux groupes. Concernant le polymorphisme de la TATAbox, il existe une réduction significative de la fréquence de l'allèle A(TA)7TAA homozygote dans la population des patients par rapport au groupe des témoins. Concernant le polymorphisme du PBREM, nous avons observé chez les témoins un lien d'exclusion entre l'allèle A(TA)7TAA de la TATAbox et l'allèle G. En revanche, il a été mis en évidence chez les patients une augmentation significative des associations A(TA)6TAA et allèle T et A(TA)7TAA et allèle G. Sur les 103 patients, une anomalie génétique dans le gène UGT1A1 pouvant être à l'origine de l'hyperbilirubinémie n'a été retrouvée que pour 52 cas. Ces résultats montrent que l'étude limitée au promoteur du gène UGT1A1 ne permet pas d'apporter une réponse suffisante en matière de diagnostic étiologique. De plus, ces résultats suggèrent une absence d'implication dans le phénotype hyperbilirubinémie néonatale des gènes OATP2 et de l'hème-oxygénase 1. Le PBREM semble être un polymorphisme influant mais son rôle doit encore être évalué sur une population plus importante. D'autres facteurs, comme par exemple l'allaitement maternel, ont probablement une implication plus importante que les aspects moléculaires dans l'origine des ictères en période néonatale.

Mots-clés : hyperbilirubinémie, UGT1A1.

IP389. MISE AU POINT DE LA QMPSF DANS LE DIAGNOSTIC MOLÉCULAIRE RÉFRACTAIRE DE LA GLYCOGÉNOSE DE TYPE IA

F. Parisot (1), A. Hubert-Buron (1, 2), L. Capel (1), P. Saugier-Weber (3), P. Labrune (2), F. Petit (1)

(1) Service de biochimie et hormonologie, unité de génétique, Hôpital AntoineBéclère (APHP), Clamart, France ; (2) Service de pédiatrie, Centre de référence maladies héréditaires du métabolisme hépatique, Université Paris Sud, INSERM U 948, Hôpital Antoine-Béclère (APHP), Clamart, France ; (3) Service de génétique, Hôpital Charles Nicolle, Rouen, France Contact : francois.petit@abc.aphp.fr

Introduction. La glycogénose de type Ia (GSDIa) ou maladie de Von Gierke est une affection rare (1 naissance sur 100000) du métabolisme du 
glycogène liée à un déficit en glucose-6-phosphatase. Elle se manifeste dès les premiers jours de vie par une intolérance au jeûne pouvant être à l'origine d'hypoglycémies graves. Elle est également caractérisée par des perturbations du métabolisme des lipides avec notamment une hypertriglycéridémie, une hépatomégalie majeure avec un risque d'adénomes hépatiques pouvant dégénérer. De nombreuses anomalies de séquence dans le gène G6PC ont été rapportées chez les patients présentant une GSDIa, la plus fréquente dans la population caucasienne étant la p.Arg83Cys. Cependant, quelques patients dont le diagnostic de GSDIa a été confirmé par enzymologie sur biopsie hépatique restent réfractaires au diagnostic moléculaire utilisant le séquençage systématique des 5 exons du gène G6PC. Le but de cette étude était de mettre en place la QMPSF (quantitative multiplex PCR of short fragments) dans le cadre du diagnostic de la GSDIa pour rechercher des délétions ne pouvant être mises en évidence par des méthodes de PCR classiques. Matériels et méthodes. La mise au point de la QMPSF pour le gène G6PC a été réalisée sur des témoins adultes sains et sur 10 patients présentant une GSDIa réfractaire au séquençage systématique des 5 exons. La PCR multiplex a été réalisée à partir de $75 \mathrm{ng}$ d'ADN en utilisant des amorces marquées au 6-FAM. Les amplicons couvraient soit une partie de l'exon soit une des jonctions intron-exon selon la taille des exons. Les amplicons ont été purifiés et séparés sur l'analyseur de fragments ABI Prism 3130 (Applied Biosystems). Les fragments d'intérêt ont été repérés grâce au marqueur de taille GS500ROX (Applied Biosystems). Discussion - conclusion. La QMPSF permet un screening rapide de larges délétions génomiques, constituant une alternative à la PCR quantitative. Son application au gène G6PC permettra d'évaluer la fréquence des grandes délétions dans ce gène et ainsi d'apporter une réponse complémentaire aux patients et aux familles pour lesquelles le diagnostic moléculaire classique n'est pas complet.

Mots-clés : QMPSF, glycogénose Ia.

口P390. VICTOIRES ET IMPASSES DU DIAGNOSTIC PHÉNOTY. PIQUE DE CERTAINES MALADIES GÉNÉTIQUES : À PROPOS DE 12 CAS DE TYROSINEMIE HÉRÉDITAIRE

L. Yargui, D.J. Ghriss, H. Benghezel, A. Berhoune

Laboratoire Central de Biochimie, CHU Mustapha, Alger, Algérie Contact : Iyecebiochem@yahoo.fr

Les erreurs innées du métabolisme des acides aminées sont une pathologie souvent rencontrée dans notre population. C'est ainsi que depuis plusieurs décennies, nous avons eu à répondre à plusieurs demandes de diagnostic de maladies génétiques et en particulier le syndrome d'hypertyrosinémie. Nous avons alors mis en pratique un screening basé sur des réactions simples de mise en évidence de l'accumulation des métabolites urinaires de la tyrosine ainsi que la mesure plasmatique de cet acide aminé aromatique. Sur un total de 657 enfants examinés, âgés de 2 à 14 mois, le diagnostic de tyrosinémie héréditaire (TH) a été retenu chez 12 malades. Seul le diagnostic phénotypique basé sur des réactions d'orientation simples et la détermination quantitative de la tyrosine ont été utilisés. Au regard des signes d'appel et de la symptomatologie clinique présentés par ces nourrissons, la forme hépatorénale (TH type 1) a été retenue. Le typage par la mesure du succinylacétone urinaire et l'évaluation de l'activité enzymatique tissulaire déficitaire n'ont pu être effectués faute de moyens techniques plus élaborés. Nous développerons, les forces des tests biochimiques simples pratiqués et leurs limites à défaut du diagnostic moléculaire non encore réalisable dans notre laboratoire.

Mots-clés : hypertyrosinémie, diagnostic phénotypique, aminoacidopathie.

aP391. HYPERGLYCINÉMIE SANS CÉTOSE : ÉTUDE RÉTROSPECTIVE DE 218 PATIENTS

C. Acquaviva, S. Boyer, P. Clerc-Renaud, C. Vianey-Saban

Service Maladies Héréditaires du Métabolisme et Dépistage Néonatal, Centre de Biologie et de Pathologie Est, CHU Lyon, France

Contact : cecile.acquaviva-bourdain@chu-lyon.fr

L'hyperglycinémie sans cétose (NKH) est une anomalie héréditaire du métabolisme de la glycine, transmise selon un mode autosomique récessif. Elle est la conséquence d'un déficit du système de clivage de la glycine (SCG), complexe enzymatique mitochondrial composé de 4 protéines : protéine $\mathrm{P}$, $\mathrm{T}, \mathrm{H}$ et $\mathrm{L}$, codées respectivement par les gènes GLDC, AMT, GCSH et DLD. Chez tous les patients décrits dans la littérature, le déficit enzymatique résulte de mutations dans les gènes GLDC ou AMT, à l'exception de 3, atteints d'une forme transitoire de NKH, pour lesquels des anomalies du gène GCSH ont été rapportées. La protéine L étant commune à d'autres systèmes multienzymatiques, la présentation clinique de ce déficit est différente de celle des patients atteints de NKH. Depuis 1986, nous avons étudié 218 patients (issus de 214 familles, d'origine caucasienne pour la majorité) atteints de NKH par mesure de l'activité du SCG et/ou étude des gènes AMT et GLDC. Les symptômes (hypotonie et convulsions) sont apparus au cours de la $1^{\text {re }}$ semaine de vie pour la majorité des patients $(84 \%)$. Seuls $5 \%$ ont développé des signes cliniques (retard psychomoteur) après l'âge de 10 mois. Le pronostic est sévère: $48 \%$ des patients décèdent avant l'âge de 1 mois et seulement $10 \%$ sont en vie à 5 ans. La chromatographie des acides aminés dans le plasma et le LCR permet d'orienter le diagnostic. Les concentrations de glycine dans le plasma varient de 177 à $3785 \mu \mathrm{mol} / \mathrm{L}$ (médiane 1125 -valeurs usuelles $<240$ ), dans le LCR de 16 à $702 \mu \mathrm{mol} / \mathrm{L}$ (médiane 179 -valeurs usuelles $<10$ ) et le ratio LCR/plasma est compris entre 0,03 et 0,61 (médiane 0,16-valeurs usuelles $<0,04$ ). Pour 5 patients, le ratio LCR/ plasma est normal mais tous présentent une concentration de glycine plasmatique supérieure à $624 \mu \mathrm{mol} / \mathrm{L}$. Le diagnostic de NKH a été confirmé par la mesure de l'activité du système de clivage de la glycine dans le foie $(45 \%$ des patients), par étude des gènes AMT et/ou GLDC (20\%) ou par les deux techniques $(35 \%)$. L'activité du SCG varie de 0 à $70 \%$ des témoins (valeur médiane $2 \%$ ), $85 \%$ des patients ayant une activité résiduelle inférieure à $15 \%$. L'analyse moléculaire a été réalisée pour tous les patients avec une activité résiduelle supérieure à $30 \%(\mathrm{n}=9)$. Pour 117 familles, le génotype a été établi : $35 \%$ des mutations ont été identifiées dans le gène AMT (35 mutations différentes dont 32 nouvelles et 1 mutation majoritaire, p.Arg 320 His concernant $23 \%$ des allèles) et $65 \%$ dans le gène GLDC (95 mutations différentes dont 50 nouvelles). Quatre vingt dix neuf familles ont bénéficié d'un diagnostic prénatal (194 au total) par technique enzymatique ou moléculaire. Cependant, une activité normale du SCG dans le tissu trophoblastique a été mesurée chez 3 fotus atteints. Il est donc indispensable de coupler l'étude génétique à la mesure de l'activité enzymatique chez tous les nouveaux patients diagnostiqués afin de proposer aux familles un diagnostic prénatal fiable par étude génétique.

Mots-clés : hyperglycinémie sans cétose, gène GLDC, gène AMT.

GP392. DÉVELOPPEMENT D'UN OUTIL BIOINFORMATIQUE (HPRED) DÉDIÉ À LA PRÉDICTION DES CONSÉQUENCES DES MUTATIONS DU GENE DE L'HYPOPHOSPHATASIE POUR L'AIDE AU DIAGNOSTIC ET AU CONSEIL GÉNÉTIQUE

H. Countouris (1), D. Fauvert (1, 2), A. Taillandier (1), I. Brun-Heath (2), P. De Mazancourt (2), E. Mornet $(1,2)$

(1) Laboratoire SESEP, Centre Hospitalier de Versailles, Le Chesnay, France : (2) EA2493, Université de Versailles Saint-Quentin en Yvelines, Versailles, France

Contact : emornet@labosesep.fr

Un nombre croissant de travaux est consacré à la construction de modèles in silico permettant de prédire les conséquences des variations de séquence au niveau de l'ADN. Ces outils sont essentiellement basés sur l'alignement multiple de séquences entre différentes espèces, sur les caractéristiques physico-chimiques des acides aminés composant la protéine et/ou, sur l'altération de structures à partir de modèles tridimensionnels. Bien que ces outils soient de plus en plus utilisés, leurs résultats de prédiction sont souvent discordants. De plus, ces outils étant destinés à l'étude de tout gène, ils ne prennent pas en compte certaines spécificités déterminées par des données expérimentales. Nous avons développé HPRED, un outil prédictif de la sévérité des mutations du gène de la phosphatase alcaline tissu non spécifique (ALPL; MIM 171760) responsables de l'hypophosphatasie, maladie héréditaire rare caractérisée par un défaut de minéralisation osseuse et dentaire. Pour ce faire nous avons généré systématiquement toutes les mutations possibles pour tous les acides aminés de la protéine. Le serveur HPRED joue d'une part le rôle d'un métamoteur capable d'afficher les scores de sévérité donnés par trois des principaux serveurs existants (SIFT, PMUT, POLYPHEN), et d'autre part d'un outil prédictif produisant un score de sévérité propre aux résultats expérimentaux obtenus au laboratoire (score SESEP). Ces derniers sont issus d'expériences de mutagenèse dirigée qui ont permis de définir des points chauds pour l'effet dominant négatif, et de l'étude des domaines fonctionnels basés sur le modèle tridimensionnel de la phosphatase alcaline. L'outil intègre également le programme de prédiction ESEfinder 3.0 pour l'identification de sites potentiellement activateurs d'épissage. Pour chaque mutation soumise par l'utilisateur, un traitement statistique des 4 scores obtenus (SIFT, PMUT, POLYPHEN et SESEP) est réalisé afin de générer un score global et unique. Afin de valider le modèle nous avons testé 67 mutations fauxsens du gène ALPL préalablement identifiées et pour lesquelles le degré de sévérité était déterminé avec certitude. Le taux de discordance entre les trois serveurs SIFT, PMUT et POLYPHEN était de $46,3 \%$. Le taux d'assignements corrects était de $68,6 \%$ pour SIFT, $55 \%$ pour PMUT, $80,5 \%$ pour POLYPHEN et $89,8 \%$ pour le serveur HPRED. En conclusion, HPRED propose un service en ligne comprenant l'analyse de mutations et/ou polymorphismes selon les coordonnées génomiques, protéiques ou 
ADNc et fournit en sortie les scores prédictifs de SIFT, PMUT, POLYPHEN et SESEP, le score prédictif global, un ensemble d'informations répertoriées dans les bases de données NCBI, ENSembl et Applied Biosystems, et la localisation du résidu muté sur le modèle tridimensionnel. L'adresse du serveur HPRED est: http://www.sesep.uvsq.fr/03 hypo_00_hpred_form.php

Mots-clés : modèle prédictif, mutation, hypophosphatasie.

DP393. PREMIÈRE OBSERVATION D'UN SYNDROME DE GÈNES CONTIGUS IMPLIQUANT UN GENE DU COMPLEXE PYRUVATE DÉSHYDROGÉNASE

A. Boutron (1), C. Thauvin-Robinet (2), C. Altuzarra (3), O. Dulac (4), P. Callier (2), F. Mugneret (2), M. Brivet (1)

(1) Laboratoire de Biochimie, AP-HP Hôpital de Bicêtre, Le KremlinBicêtre, France ; (2) Génétique, Hôpital d'Enfants de Dijon, France ; (3) Pédiatrie, CHU de Besançon, France ; (4) Neuropédiatrie, AP-HP hôpital Necker-Enfants Malades, Paris, France

Contact : michele.brivet@bct.aphp.fr

Le déficit en pyruvate deshydrogénase (PDH) atteint le métabolisme énergétique en provoquant des encéphalopathies dégénératives. Une dizaine de cas de déficits en PDH sont diagnostiqués par an en France, surtout dus à une anomalie du gène PDHA1 (Xp22.1) codant la sous-unité Elalpha. Nous avons identifié une délétion complète du gène PDHA1, hétérozygote, chez une jeune fille de 18 ans, confirmée par hybridation génomique in situ (FISH) et hybridation génomique comparative (CGH-array). Cette dernière a révélé qu'il s'agissait en fait d'un syndrome de gènes contigus impliquant 19 gènes. Cas clinique : La patiente a présenté des symptômes évocateurs d'un déficit en PDH dès son $8^{c}$ jour (mauvaise prise de poids, dilatation des ventricules cérébraux, augmentation du lactate et du pyruvate dans le sang et le LCR avec rapports L/P normaux). Un traitement par régime cétogène et thiamine a corrige les anomalies biologiques mais n'a pas empêché la survenue d'un retard du développement. L'état clinique s'est ensuite progressivement détérioré (comportement psychotique, dystonie, syndrome de West) malgré le traitement. L'activité PDH était normale dans les lymphocytes mais abaissée dans les fibroblastes, avec diminution de la sous-unité Elalpha en Western-blot, justifiant la recherche d'anomalies du gène PDHA1. Matériel et Méthodes; Le gène PDHAl a été amplifié par PCR longue (PCRL) entre le promoteur et l'extrémité 3' UTR du gène puis séquencé au niveau du promoteur et de chaque exon. Un dosage génique des extrémités du gène a été réalisé par qPCR de 2 fragments d'ADN chevauchant les amorces de PCRL. Une délétion du chromosome X a aussi été recherchée, à l'aide de sondes FISH. Les bornes de la délétion ont été déterminées à l'aide d'une puce à oligo nucléotides $244 \mathrm{k}$ Agilent. Résultats : le séquençage du promoteur de PDHA l a révélé la présence d'un changement de base, apparemment homozygote, pouvant refléter l'existence d'une grande délétion hétérozygote; la qPCR a montré qu'il n'existait qu'une seule copie du gène dans les régions 5'UTR et 3'UTR du gène. La FISH et la CGH-array ont confirmé l'existence d'une délétion intersticielle en Xp22.12 de $3,4 \mathrm{Mb}(16,42-19,81 \mathrm{Mb})$, emportant 19 gènes allant de CPTS2 à SH3KBP autour de PDHA1. Discussion : Le tableau clinique de notre patiente n'intègre pas tous signes que l'on pourrait attendre à la suite de la perte de 19 gènes contigus du chromosome $\mathrm{X}$; Il semble plutôt à rapporter au seul déficit en PDH, mis à part l'épilepsie résistante qui pourrait aussi être associée au gène CDKL5. Cette situation s'explique probablement par un biais d'inactivation du chromosome $\mathrm{X}$ favorable. Il est à noter que cette patiente est issue d'une grossesse gémellaire monochoriale monoamniotique. Sa sæur jumelle est décédée précocement au 8ème jour, dans un tableau évoquant aussi un déficit en PDH, ce qui peut suggérer qu'il existait une assymétrie d'inactivation du chromosome X, différente entre les 2 jumelles.

Mots-clés : PDH, grande deletion, gènes contigus.

口P394. LA SÉVÉRITÉ CLINIQUE DES ENCÉPHALOMYOPATHIES SECONDAIRES À DES MUTATIONS DU GÈNE SUCLGI SERAIT CORRÉLÉE À LA DIMINUTION D'EXPRESSION DE LA PROTÉINE

C. Rouzier (1, 2), S. Tuffery-Giraud (3), K. Fragaki (1, 2), V. Serre (4), J. Miro (3), S. Leguedard (3), A. Chaussenot (1), S. Bannwarth $(1,2)$, C. Caruba (5), E. Ostergaard (6), J.F. Pellissier (7), C. Richelme (8), C. Espil (9), B. Chabrol (10), V. Paquis-Flucklinger $(1,2)$

(1) Service de génétique médicale, Hôpital Archet 2, CHU de Nice, France; (2) IGMRC, FRE CNRS/UNSA 3086, Université de Nice Sophia-Antipolis, France; (3) INSERM U827, Institut de recherche clinique, Montpellier, France; (4) INSERM U781, Hôpital Necker Enfants Malades, Paris, France ; (5) Service de biochimie, Hôpital Pasteur, CHU de Nice, France ; (6) Department of Clinical Genetics, National University Hôpital Rigshospitalet, Copenhagen, Denmark; (7) Laboratoire de neu- pédiatrie, Hôpital Archet 2, CHU de Nice, France; (9) Service de Neuropédiatrie, CHU Pellegrin, Bordeaux, France; (10) Service de Neuropédiatrie, Hôpital Timone, CHU de Marseille, France

Contact : cecilerouzier@gmail.com

Les mitochondriopathies secondaires à une déplétion de l'ADN mitochondrial (ADNmt) forment un groupe hétérogène de pathologies sévères de transmission autosomique récessive. Récemment des déficits de la ligase succinate- $\mathrm{CoA}$ ont été impliqués dans des encéphalomyopathies avec déplétion de l'ADNmt et acidurie méthylmalonique modérée. À ce jour, des mutations du gène SUCLA2, qui code pour la sous-unité beta de la ligase succinate-CoA, ont été identifiées chez 17 patients. En revanche des mutations du gène SUCLG1, qui code pour la sous-unité alpha de l'enzyme, ont été rapportées dans seulement deux familles. Nous rapportons les cas de deux patients non apparentés chez lesquels nous avons identifié trois nouvelles mutations pathogènes du gène SUCLG1. Le premier patient présentait une hypotonie néonatale sévère associée à une acidose lactique et une acidurie méthylmalonique ayant entraîné le décès à l'âge de un an. Nous avons identifié chez ce patient une mutation faux-sens hétérozygote (p.Prol70Arg) et une mutation qui entraîne l'épissage complet de l'exon 1 du transcrit. Le rôle de SUCLG1 a été confirmé par les résultats du western blot qui mettait en évidence une absence totale de protéine SUCLG1 dans les fibroblastes. Le second patient, âgé de 12 ans, avait un phénotype plus modéré, similaire à celui présenté par les patients porteurs de mutations dans le gène SUCLA2. Nous avons identifié chez ce patient une mutation non-sens hétérozygote (p.Gln150X). Le western blot mettait en évidence une expression résiduelle de SUCLG1 dans les fibroblastes de ce patient. Nos résultats suggèrent qu'une absence totale de protéine SUCLGl est responsable de formes sévères d'encéphalomyopathie avec un début très précoce, voire anténatal, alors qu'une expression résiduelle de SUCLGI est impliquée dans les formes de début plus tardif de type SUCLA2-like. D'autre part, nous montrons l'absence de protéine SUCLA2 par western blot dans les fibroblastes n'exprimant pas SUCLG1. Ces résultats suggèrent une dégradation de SUCLA2 lorsque SUCLG1, son partenaire hétérodimérique, est absent.

Mots-clés : SUCLG1, mitochondriopathie, acidurie méthylmalonique.

\section{"P395. DÉFICIT EN PYRUVATE DÉSHYDROGENASE DU À UNE NOUVELLE MUTATION DU GĖNE PDHX CHEZ DEUX PATIENTS MAROCAINS}

M. Tajir (1), J.B. Arnoux (2), A. Boutron (3), S. Chafai Elalaoui (1), P. De Lonlay (2), A. Sefiani (1, 4), M. Brivet (3)

(1) Département de Génétique Médicale, Institut National d'Hygiène, Rabat, Maroc; (2) Centre de Référence Maladies Métaboliques, AP-HP Hôpital Necker Enfants-Malades, Paris, France; (3) Biochimie métabolique, AP-HP Hôpital de Bicêtre, le Kremlin-Bicêtre, France ; (4) Centre de Génomique Humaine, Faculté de Médecine et Pharmacie, Université Mohamed V Souissi, Rabat, Maroc

Contact : mariam_tajir@yahoo.fr

Le déficit en pyruvate déshydrogénase (PDH) est une maladie héréditaire du métabolisme énergétique affectant principalement le système nerveux et caractérisée par une détérioration neurologique de gravité variable. $\mathrm{La}$ PDH est un complexe enzymatique formé de 5 sous-unités (E1alpha, E1beta, E2, E3 et E3BP), codées chacune par des gènes différents et associées à une PDH kinase et une PDH-phosphatase. $\mathrm{La}$ PDH joue un rôle important dans le métabolisme du glucose en catalysant la décarboxylation oxydative du pyruvate, produit de la glycolyse, en acétyl CoA, qui rentre dans le cycle de Krebs. On décrit trois phénotypes cliniques: une forme néonatale sévère caractérisée par une détresse neurologique et une acidose lactique ; une forme infantile avec hypotonie et retard psychomoteur; une forme intermédiaire avec des épisodes d'ataxie à rechute, aggravés par les apports en sucres. Une hyperlactatémie avec un rapport lactate/pyruvate normal est retrouvée dans les 3 types. La presque totalité des mutations est retrouvée sur le gène PDHAl porté par le chromosome X, codant la sous-unité Elalpha de l'enzyme. Des mutations de transmission autosomique récessive, affectant les autres sous unités du complexe ont également été caractérisées sur le gène PDHX (en $11 \mathrm{pl} 3$; codant la protéine X ou E3BP), le gène PDHB (en 3p21.1-p14.2; codant la protéine E1 beta), le gène DLAT (en 11q23.1; codant la protéine E2) et le gène DLD (en 7q31-q32; codant la protéine E3). Nous rapportons dans ce travail les observations de deux patients d'origine marocaine ayant un déficit en PDH. La première observation décrit une forme infantile, d'un enfant âgé de 8 ans, consanguin, adressé à la consultation de génétique médicale pour fatigabilité et retard des acquisitions psychomotrices. Une hyperalaninémie à la chromatographie des acides aminés plasmatiques, l'augmentation du lactate et du pyruvate sanguin et l'IRM cérébrale étaient en faveur d'une maladie du métabolisme énergétique. L'étude de l'activité enzymatique sur fibroblastes a objectivé une baisse de l'activité de la pyruvate 
déshydrogénase et l'étude moléculaire a révélé une nouvelle mutation au niveau du gène PDHX à l'état homozygote $(c .1182+2 \mathrm{~T}>\mathrm{C})$. La deuxième observation décrit une forme néonatale, d'un nourrisson de sexe féminin, née dans un contexte de souffrance fotale aigue, qui a présenté en période néonatale une hyperlactatémie constante avec rapport lactate/pyruvate normal, une lactaturie élevée, une hyperalaninémie et des anomalies à l'IRM cérébrale. Le dosage de l'activité enzymatique sur lymphocytes a retrouvé un déficit en PDH. L'étude moléculaire a retrouvé la même mutation au niveau du gène PDHX. La récurrence de cette nouvelle mutation chez deux patients marocains non apparentés suggère un effet fondateur de cette mutation. Si cette hypothèse est confirmée, le diagnostic moléculaire du déficit en PDH pourra être débuté par la recherche de cette mutation en premier chez les patients marocains.

Mots-clés : déficit en PDH, gène PDHX, nouvelle mutation.

\section{-P396. UNE MUTATION FRÉQUENTE DANS LES DÉFICITS DU COMPLEXE PYRUVATE DÉSHYDROGÉNASE ?}

H. Romdhane (1), M.C. Marden (2), A. Boutron (1), M. Zater (1), F. Sedel (3), H. Ogier de Baulny (4), F. Rivier (5), A. Lebas (6), M. Brivet (1) (1) Laboratoire de Biochimie, AP-HP Hôpital de Bicêtre, Le KremlinBicêtre, France; (2) INSERM U779, Université Paris XI, Le KremlinBicêtre, France; (3) Neurologie, AP-HP GH Pitié-Salpêtrière, Paris, France ; (4) Centre de Référence des Maladies Métaboliques Héréditaires, Hôpital Robert Debré, Paris, France ; (5) Neuropédiatrie, Hôpital Gui de Chauliac, Montpellier, France; (6) Neuropédiatrie, AP-HP Hôpital de Bicêtre, Le Kremlin-Bicêtre, France

Contact : michele.brivet@bct.aphp.fr

Les anomalies du complexe pyruvate deshydrogénase ( $\mathrm{PDH}$ ) perturbent gravement le métabolisme énergétique et provoquent des encéphalopathies dégénératives. Une dizaine de cas de déficits en PDH sont diagnostiqués par an en France, essentiellement dus à une anomalie du gène PDHA1 codant la sous-unité Elalpha. Les anomalies des gènes codant les autres sous-unités (PDHB, PDHX, DLAT et DLD) sont extrêmement rares et sont des mutations uniques jusqu'à maintenant. C'est pourquoi l'identification d'une même mutation faux sens ( $\mathrm{p} . \mathrm{M} 101 \mathrm{~V})$ du gène PDHB codant la sous-unité Elalpha chez 6 patients, nous a conduit à nous interroger sur le caractère délétère de cette mutation. Patients : Les 6 patients ont été identifiés au sein d'une cohorte de 75 patients atteints d'un déficit en PDH. Ils présentaient une forme clinique modérée avec des accès d'ataxie à répétition et une augmentation parallèle du pyruvate et du lactate dans le sang et le liquide céphalo-rachidien au moment des crises. La diminution de l'activité PDH a été objectivée dans les lymphocytes. La recherche d'une mutation sur les 5 gènes codant les différentes sous-unités du complexe PDH n'a révélé aucune anomalie sauf sur le gène PDHB : cinq des patients (consanguins, d'origine maghrébine) étaient homozygotes pour la mutation c.301A $>\mathrm{G}$ (p.M101V) et le $6^{\mathrm{e}}$ était hétérozygote composite pour cette mutation et l'anomalie d'un site consensus d'épissage (c.301A $>$ G /c.793-2A >G). Matériel et Méthodes: Le caractère délétère de la mutation p.M101V a été étudié in silico et expérimentalement, au niveau de ses conséquences possibles sur l'épissage (séquençage du cDNA), sur la stabilité (western blot) ou la structure de la protéine (modélisation). Résultats : La mutation p.M101V concerne un acide aminé conservé chez les eucaryotes. Elle n'a pas été retrouvée sur 100 chromosomes témoins. Cette mutation fait partie du site donneur d'épissage de l'intron 5 mais ne modifie pas le cDNA-PDHB. Le western-blot réalisé à partir d'un homogénat de fibroblastes, n'a pas montré de diminution de la sous-unité Elalpha. La mutation p.M101V se situe dans une zone étroite d'interface hydrophobe, rigide, entre les sous-unités Elalpha et Elbeta.Le changement de taille lié au remplacement d'un résidu méthionine par un résidu valine (perte de 2 atomes de la chaîne latérale) pourrait destabiliser localement l'hétérodimère. Conclusions: Plusieurs données épidémiologiques et structurales suggèrent que la mutation p.M101V du gène PDHB est bien responsable du déficit en PDH des patients. De plus, cette mutation a été retrouvée récemment chez un patient autrichien à l'état hétérozygote composite (p.M101V /p.R105Q). Il n'a été rapporté dans la littérature que 9 mutations de PDHB chez 7 patients. La mutation p.M101V peut donc être considérée comme la mutation fréquente de ce gène et suggère un effet fondateur au Maghreb.

Mots-clés : pyruvate deshydrogénase, PDHB, mutation fréquente

EP397. CARTOGRAPHIE PAR HOMOZYGOTIE AVEC UNE PUCE À SNP CHEZ 3 PATIENTS AYANT UNE ANOMALIE DU TRANSPORT MITOCHONDRIAL DU PYRUVATE

M. Brivet (1), A. Boutron (1), G. Touati (2), L. Burglen (3), P. De Lonlay (2), J.L. Mandel (4)
(1) Laboratoire de Biochimie, AP-HP Hôpital de Bicêtre, Le KremlinBicêtre, France ; (2) Centre de Référence Maladies Métaboliques, AP-HP Hôpital Necker-Enfants Malades, Paris, France; (3) Génétique, AP-HP Hôpital Trousseau, Paris, France ; (4) Institut de Génétique et de Biologie Moléculaire et Cellulaire, Strasbourg, France

Contact : michele.brivet@bct.aphp.fr

Le transport du pyruvate dans la mitochondrie est une étape essentielle de la glycolyse, de la lipogénèse et de la néoglucogénèse. Un gène de la levure (YIA6) a été identifié en 2003 pour ce transporteur. Une anomalie du transport du pyruvate dans la mitochondrie a pu être caractérisée chez 2 patients non apparentés et un fœtus, dans 2 familles algériennes consanguines. Mais l'étude de 3 gènes candidats, codant pour les protéines humaines les plus homologues du produit de YIA6, n'a pas révélé d'anomalies chez ces sujets. En 2008, Le gène YIA6 a d'ailleurs été ré-attribué au transport du NAD+. Une autre approche, de cartographie par homozygotie à l'aide de puces SNP, a donc été tentée pour identifier d'autres gènes candidats. Patients : Les patients présentaient une encéphalopathie apparue dès la période néonatale, une dysmorphie faciale et une microcéphalie ainsi qu'une augmentation permanente du lactate et du pyruvate sanguins avec un rapport L/P normal. Ceci suggérait l'hypothèse d'un déficit en pyruvate deshydrogénase (PDH). Toutefois les mesures de la décarboxylation du pyruvate $(1-14 \mathrm{C})$ en $14 \mathrm{CO} 2$ à partir d'homogénats de lymphocytes ou de fibroblastes n'ont pas révélé de déficit en PDH: alors que les mêmes mesures, effectuées à partir de fibroblastes perméabilisés par la digitonine pour permettre au pyruvate de traverser la membrane plasmique et d'arriver à la mitochondrie intacte, ont montré l'absence quasi totale de décarboxylation du pyruvate (1-C14) et l'absence de transformation du pyruvate (2-14C) en acétylcarnitine ou en dérivés du cycle de Krebs. Ces anomalies ont pu être reproduites chez des témoins, en utilisant un inhibiteur spécifique du transport mitochondrial du pyruvate, l'alpha-cyano-4-hydroxycinnamate. L'anomalie du transport mitochondrial du pyruvate a été recherchée au cours de 3 grossesses ultérieures dans l'une des 2 familles, en utilisant des amniocytes perméabilisés et retrouvée chez l'un des 2 fætus d'une grossesse gémellaire. Matériel et Méthodes: Un cartographie par homozygotie a été réalisée à l'aide de puces à SNP 250K Affymetrix en utilisant l'ADN des 2 patients non apparentés et celui du fœtus atteint. Les régions d'homozygotie ont été définies par la présence de plus de 35 SNP homozygotes consécutifs et analysées grâce au logiciel HomoSNP (plewniak@igbmc.u-strasbg.fr). Résultats : Nous avons identifié quelques régions d'homozygotie et notamment une région commune de 37 SNPs homozygotes. Un gène candidat est à l'étude dans cette zone. Discussion : L'approche de cartographie par homozygotie se développe de plus en plus pour identifier les gènes responsables d'anomalies autosomiques récessives dans des familles consanguines. Elle permet une investigation, même s'il n'existe qu'un petit nombre de familles disponibles et de petite taille. II serait intéressant de savoir si d'autres cas présumés d'anomalie du transport mitochondrial du pyruvate existent (contacter les auteurs).

Mots-clés : transport mitochondrial du pyruvate, cartographie par homozygotie, puce à SNP.

\section{aP398. INSUFFISANCE CARDIAQUE AIGÜE FATALE AVEC DÉFICIT EN COENZYME Q10 CHEZ UN ENFANT ATTEINT D'ACIDÉMIE PROPIONIQUE}

K. Fragaki (1, 2), A. Cano (3), C. Cochaud (1), A. Chaussenot (1), C. Rouzier $(1,2)$, S. Bannwarth (1, 2), C. Caruba (4), B. Chabrol (3), V. PaquisFlucklinger $(1,2)$

(1) Service de Génétique Médicale, Centre de référence des pathologies mitochondriales, CHU de Nice, France; (2) IGMRC, FRE CNRS/UNSA 3086, Université de Nice Sophia-Antipolis, France; (3) Service de Neuropédiatrie, $\mathrm{CHU}$ de Marseille, France; (4) Service de Biochimie, CHU de Nice, France

Contact : k.fragaki@laposte.net

L'acidémie ou acidurie propionique est une affection métabolique, transmise sur un mode autosomique récessif, secondaire à des mutations portant sur les gènes des sous-unités alpha ou bêta de la propionyl-CoA-carboxylase. Le dysfonctionnement de cette enzyme provoque une $\alpha$ intoxication endogène $"$, liée à l'accumulation dans le sang de propionyl-CoA, acide propionique, kétones et autres composés intermédiaires du métabolisme, exposant ainsi les patients à un risque de défaillance vitale. Outre les décompensations métaboliques aiguës, malgré les progrès diagnostiques et thérapeutiques des dernières décennies, les complications principales de l'acidémie propionique sont les atteintes du système nerveux, les myocardiopathies et les pancréatites aiguës. Le rôle d'un déficit de la chaine respiratoire mitochondriale en tant que mécanisme secondaire à l'intoxication a été évoqué récemment. Nous rapportons pour la première fois un déficit en coenzyme Q10 (CoQ10) chez un enfant atteint d'acidémie propionique, 
brutalement décédé à l'âge de 14 ans d'une insuffisance cardiaque aigüe, en l'absence de décompensation métabolique de sa maladie. Le déficit en CoQ10 est le seul désordre de la phosphorylation oxydative qui réponde favorablement à un traitement médicamenteux. Il est donc important d'obtenir rapidement une analyse biochimique de la chaîne respiratoire mitochondriale chez tout patient atteint d'acidémie propionique, dès l'apparition de signes cliniques évoquant un déficit énergétique, afin d'identifier très rapidement les possibles déficits secondaires en CoQ10. Il est vraisemblable que le traitement puisse améliorer, voire prévenir les complications cardiaques observées chez ces patients, si le diagnostic biochimique est réalisé à temps.

Mots-clés : acidémie propionique, déficit en coenzyme Q10, maladies mitochondriales.

\section{aP399. DÉPISTAGE MOLÉCULAIRE DE 980 CAS SUSPECTÉS DE NEUROPATHIE OPTIQUE HÉRÉDITAIRE ET PUBLICA- TION DE 77 NOUVELLES MUTATIONS DU GENE OPA1}

M. Ferre $(1,2,3)$, D. Bonneau (1, 2, 3), D. Milea (4, 5), A. Chevrollier (1, 3), C. Verny (2, 6), H. Dollfus (7, 8, 9), C. Ayuso (10), S. Defoort (11, 12 , 13), C. Vignal (14), X. Zanlonghi (14, 15), J.F. Charlin (16, 17), J. Kaplan $(18,19,20)$, S. Odent $(16,21)$, C.P. Hamel $(22,23)$, V. Procaccio $(2,3$, $24,25)$, P. Reynier $(1,2,3)$, P. Amati-Bonneau $(1,3)$

(1) INSERM, U694, Angers, France ; (2) Université d'Angers, Faculté de Médecine, Angers, France ; (3) CHU d'Angers, Département de Biochimie et Génétique, Angers, France ; (4) Glostrup Hospital, Department of Ophthalmology, Glostrup, Denmark ; (5) University of Copenhagen, Copenhagen, Denmark ; (6) CHU d'Angers, Département de Neurologie, Angers, France ; (7) INSERM, Équipe Avenir 3439, Strasbourg, France ; (8) Université Louis Pasteur-Strasbourg, Faculté de Médecine, Laboratoire de Génétique Médicale, Strasbourg, France ; (9) CHRU de Strasbourg, Service de Génétique Médicale, Strasbourg, France ; (10) Fundación Jiménez Diaz, Servicio de Genética, CIBERER, Madrid, Spain; (11) CNRS, UMR 8160, Lille, France: (12) Université de Lille 2, Lille, France: (13) CHRU de Lille, Hôpital Roger Salengro, Service d'Explorations Fonctionnelles de la Vision, Lille, France; (14) Fondation Rothschild, Département d'Ophtalmologie, Paris, France, (15) Clinique Sourdille, Laboratoire d'Explorations Fonctionnelles de la Vision, Nantes, France; (16) Université de Rennes 1, Faculté de Médecine, Rennes, France; (17) CHU de Rennes, Service d'Ophtalmologie, Rennes, France ; (18) INSERM, U781, Unité de Recherches Génétique et Épigénétique des Maladies Métaboliques, Neurosensorielles et du Développement, Paris, France; (19) Université Paris Descartes, Faculté de Médecine, Paris, France; (20) AP-HP, Groupe Hospitalier Necker, Service de Génétique Médicale, Paris, France; (21) CHU de Rennes, Département de Médecine de l'Enfant et de l'Adolescent, Rennes, France ; (22) CHRU de Montpellier, Montpellier, France ; (23) Université Montpellierl et Montpellier2, Institut des Neurosciences, Montpellier, France ; (24) CNRS, UMR6214, Angers, France; (25) INSERM, U771, Angers, France

Contact:MaFerre@chu-angers.fr

Nous rapportons les résultats du dépistage moléculaire de 980 patients adressés à notre laboratoire pour suspicion de neuropathie optique héréditaire. Parmi ces patients, $588(60 \%)$ avaient une histoire familiale d'atrophie optique héréditaire alors que les 392 autres patients $(40 \%)$ n'avaient pas d'antécédents familiaux évidents de la maladie. Chez tous les patients, les dix mutations primaires de l'ADN mitochondrial (ADNmt) causant la neuropathie optique héréditaire de Leber (NOHL) ont été recherchées et les séquences codantes complètes des gènes $\mathrm{OPA} 1$ et $\mathrm{OPA} 3$, les deux gènes actuellement identifiés dans l'atrophie optique autosomique dominante (AOAD), ont été examinées. Des causes moléculaires ont été identifiées chez 440 patients ( $45 \%$ des patients examinés). Parmi eux, 295 patients $(67 \%)$ étaient porteurs d'une mutation d'OPA1, 131 patients $(30 \%)$ d'une mutation de l'ADNmt, et 14 patients $(3 \%)$, appartenant à trois familles non liées, avaient une mutation d'OPA3. Fait intéressant, une mutation d'OPAl a été trouvée dans $157(40 \%)$ des 392 cas apparemment sporadiques d'atrophie optique. Désormais, la base de donnée spécifique du gène OPA1 contient un total de 204 mutations, en incluant les 77 nouvelles mutations d'OPA1 identifiées suite à cette étude. L'analyse statistique de ce vaste ensemble de mutations nous a conduit à proposer une stratégie diagnostique pour optimiser l'étude moléculaire des neuropathies optiques. Nos résultats soulignent l'importance de rechercher les mutations de l'ADNmt responsables de la NOHL ainsi que les mutations d'OPA1 et OPA 3 dans les cas de neuropathie optique héréditaire suspectés, même en l'absence d'antécédents familiaux de la maladie.

Mots-clés : atrophie optique héréditaire, mitochondries, OPA1.
IP400. A DE NOVO MUTATION IN THE ATPASE 8 GENE IN A PATIENT WITH MITOCHONDRIAL DISORDER

E. Mkaouar-Rebai (1), F. Kammoun (2), I. Chamkha (1), N. Kammoun (1), I. Hsairi (2), C. Triki (2), F. Fakhfakh (1)

(1) Laboratoire de Génétique Moléculaire Humaine, Faculté de Médecine de Sfax, Tunisia ; (2) Service de Neurologie Infantile, CHU Hédi Chaker de Sfax, Tunisia

Contact : emna_mkaouar@mail2world.com

Mitochondrial DNA defects were known to be associated with a wide spectrum of human diseases and patients might present wide range of clinical features in various combinations. In the present study, we described a patient with psychomotor and neurodevelopmental delay, mild hyperintensity of posterior periventicular white matter, generalized clonic seizures, leukodystrophy and congenital deafness. He also suffered from a tetraplegia, with central blindness and swallowing difficulty. Brain MRI showed involvement of the interpeduncular nucleus and central tegmental tract, white matter abnormalities and cerebellar atrophy. A whole mitochondrial genome screening revealed the presence of 19 reported polymorphisms and an undescribed A to G mutation at nucleotide 8411 (p.M16V) affecting a conserved region of the mitochondrial ATPase 8 protein. This de novo mutation was detected in heteroplasmic form $(97 \%)$ and was absent in 120 controls. Thus, the m.8411 $\mathrm{A}>\mathrm{G}$ mutation could strongly be associated to the disease in the tested patient.

Mots-clés : ATPase 8 gene, mitochondrial disorder, mtDNA.

EP401. SPECTRE PHÉNOTYPIQUE DES PATIENTS AVEC ENCÉPHALOMYOPATHIE ET ACIDURIE MÉTHYLMALONIQUE PORTEURS D'UN DÉFICIT EN SUCCINYL-COENZYME A SYNTHÉTASE (MUTATIONS DES GÊNES SUCLA2 ET SUCLGI)

C. Haudry (1), V. Valayannopoulos (1), V. Serre (1), M. Barth (2), N. Boddaert (1), J.B. Arnoux (1), V. Cormier-Daire (1), M. Rio (1), D. Rabier (1), A. Vassault (1), A. Munnich (1), J.P. Bonnefont (1), P. De Lonlay (1), A. Rötig (1), A.S. Lebre (1)

(1) Université Paris Descartes, Hôpital Necker-Enfants Malades et Inserm U781 et U797, Départements de Génétique, de Radiologie pédiatrique, des Maladies Métaboliques et de Biochimie B. Paris, France ; (2) Université d'Angers, Hôpital d'Angers et Inserm U694, Département de Génétique, Angers, France

Contact : anne-sophie.lebre@nck.aphp.fr

La succinyl-Coenzyme A synthétase (SCS) est le complexe enzymatique qui catalyse la conversion du Succinyl-CoA en succinate. Chez les mammiferes, il existe deux isoformes spécifiques de l'ATP (SCS-A) ou du GTP (SCS-G). Les deux isoformes SCS-A et G sont des protéines hétérodimeriques constituées de la même sous-unité alpha mais de deux sous-unités béta différentes. Le gène SUCLG1 code pour la sous-unité alpha commune. Les gènes SUCLA2 et SUCLG2 codent pour les sous-unités béta des complexes SCS-A et SCS-G, respectivement. Des mutations des gènes SUCLG1 et SUCLA2 ont été décrites chez des patients avec encéphalomyopathie et acidurie methylmalonique (MMAurie). Le premier patient rapporté dans la littérature avec mutations SUCLG1 présentait un phénotype anténatal très sévère avec une mortalité précoce. Les patients mutés SUCLA2 et les autres patients mutés SUCLG1 avaient des présentations moins sévères avec un syndrome « Leigh-like ", une déplétion de l'ADN mitochondrial et une espérance de vie allant jusqu'à 21 ans. Dans cette étude, nous décrivons les données cliniques, radiologiques, biochimiques et génétiques concernant trois nouveaux patients avec mutations SUCLG1. Ces patients présentent une encéphalopathie, une hypotonie, une atrophie musculaire et une dystonie. Nous avons fait une étude de la littérature et avons comparé les phénotypes des patients SUCLA2 et des patients SUCLG1 déjà rapportés afin d'identifier un possible profil clinique et métabolique commun. Les patients porteurs de déficit en SCS ont un profil métabolique particulier (acidose lactique, présence d'acide méthylmalonique et de C4-dicarboxylic carnitine (C4-DC) dans les urines) et des lésions des ganglions de la base à l'IRM cérébrale. Une perte d'audition est observée dans les deux groupes de patients, mais est significativement plus observée chez les patients mutés SUCLA2. Tous les patients testés ont un déficit multiple de la chaîne respiratoire dans le muscle mais la déplétion de l'ADN mitochondrial n'est pas constante. Cette étude élargit le spectre phénotypique des mutations SUCLG1 et confirme la mise en évidence d'un profil métabolique caractéristique et d'une image très évocatrice en IRM cérébrale (lésions de ganglions de la base) dans les déficits en SCS. L'existence d'une déplétion de l'ADN mitochondrial dans le muscle n'est pas constante, ce qui permet de suggérer un mécanisme physiopathologique alternatif pour expliquer le déficit multiple de la châne respiratoire observé chez les patients.

Mots-clés : gène SUCLG1, acidurie méthylmalonique, déplétion de l'ADN mitochondrial. 
P402. DIAGNOSTIC DIFFERENTIEL DES CYTOPATHIES MITOCHONDRIALES : ENCÉPHALOPATHIE AIGUË NÉCROSANTE LIÉE AUX MUTATIONS DE RANBP2

M. Rio (1), A.S. Lèbre (1), M. Mazzuca (2), M. Willems (1), N. Brahimi (1), A. Kuster (3), M. Holder (4), A. Munnich (1), A. Rotig (1), P. De Lonlay (5), N. Boddaert (6)

(1) Département de Génétique, Hôpital Necker-Enfants Malades, Paris ; (2) Service de Neuropédiatrie, Hôpital Necker-Enfants Malades, Paris; (3) Service de Pédiatrie, CHU de Nantes, Nantes; (4) Département de Génétique, Hôpital Jeanne-de-Flandre, Lille ; (5) Service de Métabolisme, Hôpital Necker-Enfants Malades, Paris; (6) Département de Radiologie Pédiatrique, Hôpital Necker-Enfants Malades, Paris

Contact : marlene.rio@nck.aphp.fr

Affirmer le diagnostic de cytopathie mitochondriale chez l'enfant est un exercice difficile. En effet, si le diagnostic est fondé sur les résultats de l'investigation enzymologique de la chaîne respiratoire mitochondriale, il est malheureusement rarement affirmé par une étude moléculaire compte tenu de l'extrême hétérogénéité de ces maladies. Or, les signes cliniques, biologiques et radiologiques qui font suspecter le diagnostic sont souvent peu spécifiques et le diagnostic est finalement affirmé par le seul résultat de l'étude enzymologique. Toutefois, cette étude enzymologique n'affirme pas le diagnostic de cytopathie mitochondriale mais met en évidence un défaut de phosphorylation oxydative qui peut être primaire ou secondaire à une autre pathologie. Labellisé depuis 2005, le centre de Référence CARAMMEL (Centre de Référence pour les Maladies Mitochondriales de l'Enfant à l'Adulte) est régulièrement sollicité pour l'expertise de dossiers afin de valider avec certitude le diagnostic de maladie mitochondriale et répondre à la demande conseil génétique et de diagnostic prénatal des parents. Nous rapportons les observations de 3 patients français non apparentés ayant présenté plusieurs épisodes pseudo-encéphalitiques aigus déclenchés par des épisodes fébriles. Le diagnostic de cytopathie mitochondriale avait été évoqué devant cette présentation clinique, d'autant que l'étude enzymologique de la chaîne respiratoire mitochondriale était anormale pour l'un des patients. Cependant, la rapidité de progression de l'atteinte neurologique, l'existence d'une hyperprotéinorrachie, la récupération neurologique quasi complète entre deux épisodes pour un patient ont fait remettre en cause le diagnostic initialement suspecté. Notamment, l'atteinte symétrique des thalami sans atteinte des pallidi et des putamens associée à l'atteinte du tronc cérébral observée à l'IRM cérébrale, a conduit à discuter le diagnostic d'une encéphalopathie aiguë nécrosante (Acute Necrotizing Encephalopathy) en rapport avec une mutation du gène codant pour une protéine du pore nucléaire, RANBP2. L'étude du gène RANBP2 a permis de confirmer ce diagnostic en identifiant chez les 3 patients la mutation c.1880C >T à l'état hétérozygote dans l'exon 12, déjà rapportée comme récurrente dans la littérature. Ces trois observations confirment la nécessité d'une collaboration entre cliniciens, radiologues et biologistes pour l'expertise diagnostique des suspicions de cytopathies mitochondriales et soulignent l'importance de l'imagerie cérébrale qui, à l'instar de la dysmorphologie, permet d'orienter les études moléculaires avec pour corollaire l'établissement d'un diagnostic de certitude ouvrant la possibilité à formuler un conseil génétique et proposer un diagnostic prénatal.

Mots-clés : maladie mitochondriale, RANBP2, IRM.

TP403. PARAPLÉGIE SPASTIQUE À TRANSMISSION MATERNELLE ASSOCIÉE À LA MUTATION MITOCHONDRIALE M.9176T>C ET À UN DÉFAUT D'ASSEMBLAGE DE L'ATP SYNTHASE

C. Verny (1), N. Guegen (2), V. Desquiret (2), A. Prundean (1), F. Dubas (1), A. Chevrollier (2), P. Amati-Bonneau (2), D. Bonneau (2), P. Reynier (2), V. Procaccio $(2,3)$

(1) Département de Neurologie, CHU Angers, France ; (2) Département de Biochimie et Génétique, CHU Angers, France; (3) UMR INSERM U771-CNRS6214, Université d'Angers, France

Contact : viprocaccio@chu-angers.fr

Introduction : Les maladies mitochondriales dues à un déficit de la chaîne respiratoire sont une cause importante de pathologies neurologiques héréditaires. Les paraplégies spastiques se manifestent par une faiblesse musculaire progressive des membres inférieurs et une spasticité. Elles sont caractérisées par une très forte hétérogéneité sur le plan clinique et génétique. À ce jour, plus de 40 gènes responsables de paraplégies spastiques ont pu être localisés et/ou identifiés avec des formes dominantes, les plus communes mais aussi des formes autosomales récessives ou liées à I'X. Résultats : Nous présentons une famille dont 5 membres sont atteints de paraplégie spastique d'expressivité clinique variable, de forme pure ou associée à d'autres troubles neurologiques. La transmission compatible avec une hérédité maternelle nous a amené à analyser le génome mitochondrial qui s'est révélé porteur de la mutation m.9176T $>$ C située dans le gène codant la sous-unité 6 de l'ATP synthase. Cette mutation, classiquement associée au syndrome NARP (Neuropathie, Ataxie, Rétinite Pigmentaire) ou au syndrome de Leigh, en fonction de son taux d'hétéroplasmie, s'est révélée homoplasmique dans tous les tissus explorés chez les patients de cette famille. L'exploration fonctionnelle des fibroblastes des patients atteints a révélé un déficit de production d'ATP en relation avec un défaut d'assemblage de l'ATP synthase ainsi qu'une augmentation de stress oxydant mitochondrial. La sévérité de l'expression clinique étai parfaitement corrélée à l'importance du défaut métabolique. Discussion-Conclusion: Cette première description d'une paraplégie spastique héréditaire due à une mutation de l'ADN mitochondrial illustre l'extrême diversité de la présentation clinique des maladies mitochondriales, une même mutation pouvant être à l'origine de syndromes parfaitement distincts. Le caractère homoplasmique de la mutation m.9176T $>\mathrm{C}$ dans cette famille est aussi très singulier et ne permet pas de corréler la sévérité de la maladie au taux de mutant comme cela est classiquement le cas dans les maladies de l'ADN mitochondrial. Au sein de cette famille, les hommes étaient plus sévèrement touchés que les femmes, ce qui pourrait suggérer l'implication de facteurs modulateurs de l'expression clinique, tels que les facteurs hormonaux. Plusieurs gènes nucléaires codant des protéines mitochondriales (paraplégine, HSP60 et REEP1) sont aussi à l'origine de paraplégies spastiques héréditaires ce qui souligne l'importance des anomalies de la conversion énergétique dans la physiopathologie de ces maladies. Notre observation indique l'intérêt de l'étude de l'ADN mitochondrial lorsque le mode de transmission de la maladie est compatible avec une transmission maternelle et aussi dans des phénotypes neurologiques incluant les paraplégies spastiques.

Mots-clés: mitochondrie, paraplégie spastique héréditaire, $\mathrm{ADN}$ mitochondrial.

P404. RECHERCHE DE MUTATIONS MITOCHONDRIALES CHEZ UNE FAMILLE ATTEINTE DE DIABÈTE MITOCHONDRIAL: MISE EN ÉVIDENCE DE LA MUTATION T14709C À L'ÉTAT HÉTÉROPLASMIQUE

F. Fakhfakh (1), N. Mezghani (1), E. Mkaouar-Rebai (1), S. Youssef (1), M. Mnif (2), N. Charfi (2), M. Abid (2)

(I) Laboratoire de Génétique Moléculaire Humaine, Faculté de Médecine de Sfax, Tunisie ; (2) Service d'Endocrinologie, CHU Hédi Chaker, Sfax, Tunisie

Contact : faiza.fakhfakh@gnet.tn

Le diabète mitochondrial peut être associé à plusieurs signes donnant différents syndromes (MELAS, MERRF, MIDD, KSS, etc). Il est généralement associé à des mutations de l'ADN mitochondrial qui touchent essentiellement des ARNt mitochondriaux. Les mutations les plus impliquées dans le diabète mitochondrial sont la mutation $\mathrm{A} 3243 \mathrm{G}$ au niveau du gène ARNtLeu(UUR) et la mutation T14709C au niveau du gène ARNtGlu. Notre étude a porté sur une famille Tunisienne atteinte de diabète mitochondrial chez laquelle nous avons étudié les deux gènes le plus souvent impliqués dans cette forme de maladie: MT-TL et MT-TQ. Cette famille comporte 2 patientes dont la mère présente en plus du diabète, une myopathie, une obésité et un syndrome pluri métabolique. L'étude moléculaire a été effectuée sur l'ADN génomique total extrait à partir du sang selon la méthode standard. La recherche de mutations ponctuelles a été effectuée par les méthodes PCR-RFLP, PCR-SSP et par séquençage automatique. Les résultats de la PCR-RFLP et du séquençage automatique ont montré l'absence de la mutation $\mathrm{A} 3243 \mathrm{G}$ au niveau du gène $\mathrm{ARN}$ tLeu(UUR) chez les membres de la famille et la présence de la mutation T14709C au niveau du gène de l'ARNtGlu. Cette mutation a été retrouvée à l'état héteroplasmique par PCR-SSP seulement chez la mère et ses 2 filles. Les résultats retrouvés sont en faveur de l'association de la mutation T14709C au niveau du gène de l'ARNtGlu avec le diabète mitochondrial chez cette famille.

Mots-clés : diabète mitochondrial, hétéroplasmie, T14709C.

IP405. STRATÉGIE DIAGNOSTIQUE DANS LES DÉFICITS ISOLES EN COMPLEXE I DE LA CHAÎNE RESPIRATOIRE

Z. Assouline, N. Boddaert, C. Haudry, M. Jambou, M. Rio, V. Valayannopoulos, P. de Lonlay, J. Steffann, I. Desguerre, A. Munnich, J.P. Bonnefont, A. Rötig, A.S. Lebre

Université Paris Descartes, Hôpital Necker-Enfants Malades et Inserm U781 et U797, Départements de Génétique, de Radiologie pédiatrique, de Neurologie et des Maladies Métaboliques, Paris, France

Contact : anne-sophie.lebre@nck.aphp.fr. 
Les déficits isolés en complexe $\mathrm{I}(\mathrm{CI})$ de la chaîne respiratoire $(\mathrm{CR})$ sont une cause fréquente de déficit de la CR chez l'enfant. Les présentations cliniques des patients avec déficits en $\mathrm{Cl}$ sont variées (syndrome de Leigh, neuropathie optique de Leber, syndrome MELAS pour Mitochondrial Encephalomyopathy, Lactic Acidosis and Stroke-like episodes) et peuvent résulter de l'association d'une hypotonie, un retard psychomoteur, une épilepsie, une cardiomyopathie, une atrophie optique ou une rétinopathie. Le CI comprend sept sous-unités codées par l'ADN mitochondrial (ADNmt), 38 sous-unités codées par le génome nucléaire ainsi qu'un nombre croissant de facteurs d'assemblage. Jusqu'à présent, des mutations ont été identifiées dans douze gènes nucléaires (NDUFS1-4, NDUFS6-8, NDUFV1-2, NDUFA1-2 et NDUFA11), sept gènes codés par l'ADNmt et six gènes codant pour des facteurs d'assemblage (NDUFAF1-4, C8orf38 et $\mathrm{C} 20$ orf7). Le diagnostic d'un déficit isolé en CI repose sur des arguments cliniques et des résultats d'examens histologique et enzymologique du tissu atteint. Ce diagnostic est complexe du fait de l'implication de deux génomes (nucléaire et mitochondrial) et du très grand nombre de gènes potentiellement impliqués. Toute anomalie d'une sous-unité de structure ou d'assemblage du CI va entraîner un déficit primaire en CI. La perturbation d'autres fonctions mitochondriales peut être à l'origine de déficits secondaires en CI. C'est donc l'identification de mutations qui permet de confirmer le diagnostic. La tâche est immense et les progrès dans le diagnostic des déficits en CI doivent venir de la mise en évidence de corrélations clinico-biologiques et d'une évolution technologique importante $\mathrm{La}$ mise en évidence de corrélations clinico-biologiques vient du travail collectif des Centres de Référence avec la création de registres de patients mutés. Ainsi, en collectant des IRM cérébrales de patients mutés, nous avons pu identifier un pattern caractéristique des déficits primaires en $\mathrm{CI}$. Au niveau moléculaire, la mise en place de techniques performantes sont indispensables pour progresser. Ainsi, l'étude exhaustive de l'ADNmt $(16,5 \mathrm{~kb})$ est devenue possible avec la méthode Surveyor. La technique d'électrophorèse en gel non dénaturant (BN-PAGE) peut aussi être très utile. La présence de mutations dans un gène du CI peut résulter en un profil anormal avec l'accumulation d'intermédiaires d'assemblage. $\mathrm{Ce}$ profil peut aider dans le choix des gènes candidats à séquencer. Enfin, les progrès dans le diagnostic des déficits en $\mathrm{CI}$ doivent venir de l'accès à des techniques plus performantes comme le séquençage haut-débit des gènes nucléaires du CI. L'identification des bases génétiques des déficits en $\mathrm{Cl}$ est une étape indispensable de la prise en charge des patients et leur famille, afin de proposer un conseil génétique fiable et la possibilité d'un diagnostic prénatal.

Mots-clés : maladies mitochondriales, complexe I, syndrome de Leigh.

\section{-P406. CONFUSION DIAGNOSTIQUE ENTRE DES TUMEURS DE LA PLAQUE TECTALE ET DES LÉSIONS LIEES À DES MUTATIONS DU GENE MT-ND5 CODE PAR L'ADN MITO- CHONDRIAL}

A.S. Lebre, M. Rio, P. de Lonlay, V. Valayannopoulos, C. Haudry, I. Desguerre, J. Kaplan, J.L. Dufier, C. Sainte-Rose, C. Treguier, F. Brunelle, A. Rötig, A. Munnich, N. Boddaert

Université Paris Descartes, Hôpital Necker-Enfants Malades et Inserm U781 et U797, Départements de Génétique, de Radiologie pédiatrique, de Neurologie, de Neuro-Chirurgie, d'Ophtalmologie et de Maladies Métaboliques, Paris, France

Contact : anne-sophie.lebre@nck.aphp.fr

Nous rapportons l'histoire médicale de trois patients vus initialement pour suspicion de tumeur de la plaque tectale. La patiente 1 , âgée de 14 ans, a été initialement suivie pour altération de la vision et découverte fortuite à I'IRM cérébrale d'une anomalie de la plaque tectale qui a été prise pour une tumeur. Par la suite, sont apparues des difficultés scolaires, une atrophie optique, et des hypersignaux T2 au niveau du cervelet avec atrophie cérébelleuse progressive et pic de lactate à l'IRM cérébrale. Devant cette association inexpliquée, l'hypothèse d'une maladie énergétique a été soulevée et a conduit à la réalisation d'une biopsie musculaire pour étude de la chaîne respiratoire mitochondriale. Cette étude a révélé l'existence d'un déficit en complexe I de la chaîne respiratoire dans le muscle. L'étude de l'ADN mitochondrial dans le muscle de la patiente 1 par la méthode Surveyor a permis d'identifier la mutation m.13513G $>\mathrm{A}$ à l'état hétéroplasmique dans le gène MT-ND5. Cette mutation, survenue de novo, permet d'expliquer l'association de l'atrophie optique et des anomalies constatées à l'IRM cérébrale. Le patient 2 , âgé de 10 ans, a été pris en charge depuis l'âge de 4 ans pour un strabisme divergent avec amblyopie. L'IRM initiale avait montré un hypersignal périaqueducal et de la lame tectale pour lequel avait été suspectée une origine tumorale motivant un suivi radiologique qui n'avait pas montré d'évolutivité de cette image. Secondairement sont d'un pic de lactate à l'IRM cérébrale. Du fait de la similitude de cette imagerie cérébrale avec celle de la patiente 1 , l'hypothèse d'une maladie énergétique a été soulevée et une biopsie de muscle a été réalisée. Celle-ci n'a pas montrée d'anomalie de la chaîne respiratoire mitochondriale. Toutefois, devant la similitude des images radiologiques, l'étude du gène MT-ND5 a été réalisée et a permis d'identifier une mutation m.13514A $>\mathrm{G}$ à l'état hétéroplasmique. Cette mutation permet d'expliquer les difficultés praxiques, l'anomalie constatée à l'IRM cérébrale et la gène visuelle. La patiente 3, âgée de 6 ans, est une jeune fille qui présente un décalage des acquisitions fixé, une hyperlactaturie et une IRM cérébrale avec anomalie de signal péri aqueducale. Du fait de la similitude de cette imagerie cérébrale avec celle des patients 1 et 2, l'étude du gène MT-ND5 a été réalisée et a permis d'identifier une mutation m.13513G >A à l'état hétéroplasmique dans le muscle. À l'examen clinique, ces trois patients présentaient une altération de la vision et des hypersignaux de la plaque tectale à l'IRM cérébrale. Cette association inexpliquée doit faire rechercher une atrophie optique et une mutation du gène MT-ND5 codé par l'ADN mitochondrial.

Mots-clés : maladies mitochondriales, tumeur de la plaque tectale, gène MT-ND5.

P407. POLYNEUROPATHIE AMYLOÏDE FAMILIALE LIÉE À TTR: UN POLYMORPHISME MITOCHONDRIAL PEUT-IL EXPLIQUER LA DIFFÉRENCE DE PÉNÉTRANCE SELON L'ORIGINE PARENTALE DE LA MUTATION ?

B. Bonaïti $(1,2)$, M. Olsson (3), U. Hellman (3), O. Suhr (3), C. BonaïtiPellié $(2,4)$, V. Planté-Bordeneuve $(5,6)$

(1) INRA-GABI, Jouy-en-Josas, France : (2) INSERM, U535, Villejuif, France: (3) Department of Public Health and Clinical Medicine, Umeà University Hospital, Umeà, Sweden; (4) Univ Paris-Sud, Villejuif, France: (5) Département de Neurologie, CHU Henri Mondor-AP-HP, Créteil, France ; (6) Inserm U267, Institut Cochin, Paris, France

Contact : catherine.bonaiti@inserm.fr

La polyneuropathie amylö̈de liée à la transthyrétine avec mutation Val30Met (TTR-V30M-FAP) est la forme la plus fréquente d'amylö̈dose familiale, avec une transmission autosomique dominante. Cette maladie grave présente d'importantes disparités dans l'âge de survenue et la pénétrance. Récemment, une différence de pénétrance selon le sexe du parent transmetteur a été mise en évidence dans différentes régions géographiques avec une pénétrance plus élevée en cas de transmission maternelle de la mutation (Hellman et al., 2008 ; Bonaïti et al., 2009). Par ailleurs, des différences dans la distribution des haplogroupes mitochondriaux selon l'âge de début de l'affection chez des patients porteurs d'une mutation TTR-V30M-FAP en Suède et en France ont été observées suggérant qu'un polymorphisme de l'ADN mitochondrial pourrait être un mécanisme sousjacent de variation phénotypique (Olsson et al., 2009). Nous avons testé cette hypothèse en modélisant la fonction de pénétrance avec un effet « origine parentale » et/ou l'effet d'un polymorphisme mitochondrial dans des échantillons de familles ayant une TTR-V30M-FAP : 33 familles portugaises et 78 familles suédoises dans lesquelles plusieurs individus avaient été testés pour les haplogroupes mitochondriaux. Cette analyse a montré que l'effet d'un polymorphisme mitochondrial était suffisant pour expliquer la différence de pénétrance observée selon le sexe du parent transmetteur dans l'échantillon portugais, alors que, dans l'échantillon suédois, il reste clairement un effet « origine parentale » résiduel. Cette étude est en faveur du rôle d'un polymorphisme mitochondrial qui induirait une plus forte pénétrance en cas de transmission maternelle de la maladie. En pratique clinique, ces résultats pourraient permettre de mieux diagnostiquer les individus les plus à risque et modifier de façon significative la prise en charge des patients et des personnes à risque. Hellman U, Alarcon $\mathrm{F}$, Lundgren HE, Suhr OB, Bonaïti-Pellié C, Planté-Bordeneuve V. Heterogeneity of penetrance in familial amyloid polyneuropathy, ATTR Val30Met, in the Swedish population. Amyloid 2008; 15 : 181-6. Bonaîti B, Alarcon F, Bonaïti-Pellié C., Planté-Bordeneuve V. Parent-of-origin effect in Transthyretin related amyloid polyneuropathy. Amyloid 2009; 16: 149-50. Olsson M, Hellman U, Planté-Bordeneuve V, Jonasson J, Lang K, Suhr OB. Mitochondrial haplogroup is associated with the phenotype of familial amyloidosis with polyneuropathy in Swedish and French patients. Clin Genet $2009 ; 75: 163-8$.

Mots-clés : polyneuropathie amyloïde, pénétrance, mitochodrie.

GP408. GÈNES MODIFICATEURS ET EXPRESSION DE LA DRÉPANOCYTOSE : CARACTÉRISTIQUES DES PATIENTS SUIVIS AU CENTRE DE COMPÉTENCE DE BORDEAUX

E. Klein (1), S. Dulucq (1), C. Ged (2, 3), F. Moreau-Gaudry $(2,3)$, H. De Verneuil (2, 3), C. Runel (4), Y. Perel (4), M. Micheau (1, 4) 
(1) Laboratoire d'Hématologie, Hôpital Pellegrin, Bordeaux, France ; (2) Laboratoire de Biochimie et Biologie Moléculaire, Hôpital Pellegrin, Bordeaux, France ; (3) INSERM U876, Université V Segalen Bordeaux2, Bordeaux, France ; (4) Centre de compétences des maladies constitutionnelles du globule rouge et de l'érythropoïèse, Hôpital Pellegrin, Bordeaux, France

Contact : cecile.ged@u-bordeaux2.fr

Bien que définie par une mutation unique, la drépanocytose se présente cliniquement de manière très variable selon les sujets, sa sévérité variant de formes létales précoces à des formes beaucoup mieux tolérées. Cette variabilité d'expression de la maladie est expliquée en partie par l'action de gènes modulateurs. Ce travail a pour but de caractériser les patients atteints d'un syndrome drépanocytaire majeur, suivis au Centre de Compétence des Maladies Constitutionnelles du globule rouge de Bordeaux. À titre rétrospectif, une population de 50 patients (nourrissons, enfants et adultes) suivis au Centre de Compétence a été sollicitée pour participer à cette étude. Les analyses génétiques, réalisées après obtention d'un consentement éclairé auprès des sujets majeurs ou de leurs représentants légaux pour les mineurs, sont les suivantes : - détermination du polymorphisme de restriction XmnI (-158 C $>\mathrm{T}$ promoteur HBG2). - détermination des haplotypes classiques (Benin, Bantu, Cameroun, Senegal/Arab Indian) par analyse des polymorphismes Hind III (gènes HBG2 et HBG1) et Hinc II (gènes HBP1 et HBD) ou des SNP de la région amont du promoteur HBG2 $(-1450 \mathrm{~A}>\mathrm{G} ;-1280 \mathrm{G}>\mathrm{A} ;-1225 \mathrm{G}>\mathrm{A})$. - détermination du polymorphisme de répétition du promoteur du gène UGT1A1. - recherche de la présence d'une alpha-thalassémie. Les résultats de l'analyse de ces facteurs génétiques modulateurs seront mis en parallèle avec les paramètres clinico-biologiques des patients : fréquence des crises douloureuses vaso-occlusives, manifestations neurologiques, infections, syndrome thoracique aigu, ostéonécrose, lithiase vésiculaire, rétinopathie, priapisme, nombre de grossesses, taux d'hémoglobine et de leucocytes de base, taux d'hémoglobine foetale de base, activité en G6PD, bilirubine, traitement ou non par Hydrea. Cette étude a un double objectif. Dans un premier temps, dans une attitude observationnelle, de décrire la population drépanocytaire suivie au Centre de Compétence de Bordeaux. Dans un deuxième temps, la mise en application de ces analyses génétiques en pratique de routine fournirait aux médecins du Centre de Compétence un outil pronostique précieux pour le suivi de la maladie. Chaque patient pourrait ainsi bénéficier d'une étude approfondie de son profil génétique, en vue d'une amélioration de la prise en charge et de la gestion des risques de complications de la drépanocytose. Mots-clés : drépanocytose, gènes modificateurs, haplotypes.

PP409. ANALYSE DU GÈNE ABCC6 DANS UNE COHORTE DE PATIENTS DRÉPANOCYTAIRES PRÉSENTANT DES LÉSIONS ÉVOCATRICES DE PSEUDOXANTHOME ÉLASTIQUE

A.L. Fauret (1, 2), E. Sbidian (3), V. Nau (1), C. Travers (1), F. Galacteros (4), X. Jeunemaitre $(1,2), A$. Cosnes (3)

(1) AP-HP, Hôpital Européen Georges-Pompidou, Département de Génétique, Paris, France ; (2) Université Paris Descartes, Faculté de Médecine, Paris, France; (3) AP-HP, Hôpital Henri Mondor, Service de Dermatologie, Créteil, France; (4) AP- HP, Hôpital Henri Mondor, Service d'Hématologie - unité fonctionnelle: maladies génétiques du globule rouge, Créteil, France.

\section{Contact : anne-laure.fauret@egp.aphp.fr}

Introduction. Le pseudoxanthome élastique (PXE), affection héréditaire autosomique récessive, se caractérise par la destruction des fibres élastiques. Son expression clinique est cutanée, oculaire et cardiovasculaire. $80 \%$ des cas de PXE sont dus à des mutations du gène ABCC 6 codant pour un transporteur membranaire, ABCC6 ou MRP6, dont le rôle précis est inconnu. Dans la population drépanocytaire, un nombre notable de patients développe un PXE similaire au PXE classique. L'hypothèse la plus souvent proposée est celle d'un processus acquis de destruction des fibres élastiques par des phénomènes oxydatifs mais aucun génotypage n'a été réalisé. Objectif. Tester par biologie moléculaire la possibilité d'authentiques PXE chez des patients drépanocytaires avec lésions évocatrices de la pathologie. Patients et Méthodes. Au sein de la cohorte drépanocytaire de l'hôpital Henri Mondor, 15 patients avec lésions de PXE ont été sélectionnés par croisement de fichiers photographique $(n=4)$, anatomopathologique $(n=2)$ et ophtalmologique (stries angioïdes $n=5$, drüsens $n=4$ ). Après signature du consentement et extraction de l'ADN génomique, les 31 exons du gène ABCC6 ont été amplifiés puis les produits PCR analysés sur un séquenceur automatique. Résultats. L'âge moyen des patients drépanocytaires était de 39 ans. Sept présentaient des lésions cutanées typiques de PXE avec une histologie positive pour les 5 patients testés. Un seul présentait des stries angiö̈des. Les 8 autres patients avaient une atteinte oculaire isolée (stries angioïdes $\mathrm{n}=4$, drüsens $\mathrm{n}=4$ ) avec une atteinte histologique cutanée pour un des 5 sujets biopsiés. L'analyse moléculaire a révélé un patient hétérozygote composite avec lésions cutanées typiques sans atteinte oculaire: p.Leu522Pro et p.Arg887Cys non décrites dans la littérature. Trois patients présentaient une mutation hétérozygote qui a déjà été rapportée : p.Arg1314Trp pour un malade avec lésions cutanées isolées et p.Leu248Phe pour deux patients avec atteinte strictement oculaire. Discussion. En acceptant une prévalence de la maladie de 1/25000 avec une fréquence allélique attendue de $1 / 158$ et une transmission récessive, l'hypothèse d'un pseudo-PXE acquis dans la population drépanocytaire ne semble pas satisfaisante au regard de la fréquence allélique retrouvée dans cette étude, $16,7 \%$. Nous retrouvons 4 mutations dont 3 sont hétérozygotes n'éliminant pas le diagnostic. En effet, un défaut de la technique semble peu probable (sensibilité $97 \%$ ) et d'authentiques PXE ont été décrits chez des patients hétérozygotes pour $\mathrm{ABCC} 6$. L'hétérozygotie pour ABCC6 pourrait favoriser l'expression clinique du PXE dans cette population déjà soumise à des processus oxydatifs liés à leur hémoglobinopathie. Une analyse moléculaire sur une grande cohorte avec population témoin de même origine est nécessaire.

\section{口P410. DÉFICIT EN ALPHA-1 ANTITRYPSINE ET GÈNES MODI- FICATEURS}

M.F. Odou (1, 2), M. Crépin (1), L. Fruit (1, 2), G. Lalau (3), F. Zerimech (1), J. Leclerc (3), E. Crème (1), N. Porchet $(1,4,5)$, M. Balduyck $(1,2)$ (1) Laboratoire de biochimie et biologie moléculaire hormonologie métabolisme nutrition oncologie, Centre de Biologie Pathologie, CHRU, Lille, France ; (2) Faculté des Sciences Pharmaceutiques et Biologiques, Lille, France; (3) Laboratoire de biochimie, toxicologie, génopathies, Centre de Biologie Pathologie, CHRU, Lille, France; (4) Faculté de Médecine, Lille, France; (5) IMPRT IFR 114, Centre de Recherche JP Aubert INSERM, Lille, France

Contact : marie-francoise.odou@chru-lille.fr

Le déficit en alpha-1 antitrypsine (antiprotéase codée par le gène SERPINA1), est une maladie génétique autosomique récessive qui se traduit par une atteinte pulmonaire de type emphysème ou BPCO (bronchopneumopathie chronique obstructive) et parfois par des troubles hépatiques voire pancréatiques. Il existe, pour cette antiprotéase (AAT), un polymorphisme génétique important. Plus de 100 allèles différents ont été caractérisés parmi lesquels les variants PIM sont les plus fréquents dans la population caucasienne et donnent lieu à un taux circulant d'AAT et une activité antiélastasique normaux. Les variants PIS et PIZ, caractérisés par un déficit en AAT pondéral et/ou fonctionnel, sont les variants déficitaires les plus courants tandis que les variants PINuls sont caractérisés par l'absence de synthèse hépatique d'AAT. Par ailleurs, chez les sujets déficitaires, il existe encore une grande variabilité d'expression clinique, suggérant que des gènes modificateurs pourraient jouer un rôle important dans l'expression de ces pathologies. Nous avons donc recherché l'existence d'un lien entre l'expression clinique du déficit en AAT et la présence de variants alléliques sur des gènes modificateurs candidats tels ceux codant pour l'IL-10 ou le CASR (récepteur sensible au calcium). Les patients ont été répartis en quatre groupes: un groupe témoin constitué de sujets ne présentant pas de déficit en AAT $(n=28$, âge moyen 44 ans), un groupe de patients atteints d'emphysème, déficitaires en AAT PIZZ ( $\mathrm{n}=20$, âge moyen 57 ans), un groupe de patients atteints d'emphysème, hétérozygotes et possédant un allèle déficitaire du gène SERPINA1 ( $\mathrm{n}=16$, âge moyen 47 ans), et un groupe de sujets jeunes atteints de pancréatite chronique et pour lesquels une susceptibilité génétique est évoquée $(\mathrm{n}=15$, âge moyen 23 ans). Le séquençage, effectué sur séquenceur Applied Biosystems $3130 \mathrm{XL}$, a concerné l'ensemble des exons codants du gène SERPINA1, le promoteur et les exons codants du gène IL10 et un fragment de l'exon 7 du gène CASR. Puis une étude ciblée de 3 SNPs du promoteur de l'IL-10 a été effectuée par une technique de $\mathrm{SNaPshot}{ }^{\circ}$. Ce travail pourrait permettre de montrer une association entre les variants de I'AAT et les SNPs étudiés, en relation avec la sévérité des tableaux cliniques présentés. Les résultats préliminaires ne mettent pas en évidence de différences significatives dans la répartition de la fréquence des SNPs des différents groupes mais quelques tendances apparaissent, en particulier dans le groupe des patients atteints de pancréatite chronique. Ainsi, les variants allèliques de 2 SNPs de l'IL10 et 1 SNP du CASR sembleraient plus fréquemment retrouvés chez les patients atteints de pancréatite chronique que dans la population témoin. Ces résultats préliminaires nous incitent à poursuivre l'étude sur un nombre de patients plus important afin de mieux comprendre les facteurs génétiques impliqués dans ces pathologies.

Mots-clés : déficit en alpha-1 antitrypsine, gènes modificateurs, IL 10. 
D411. RECHERCHE DE GENES MODIFICATEURS DANS UNE
FAMILLE EMERY-DREIFUSS AVEC HETÉROGENÉITÉ PHÉNOTYPIQUE

B. Granger (1, 2), L. Gueneau (3, 4), V. Drouin-Garraud (4), V. Pedergnana $(3,4)$, R. Ben Yaou $(3,4), S$. Tezenas du Montcel $(1,2), G$. Bonne $(3,4,6)$

(1) Université Pierre-et-Marie-Curie Paris 06, ER4, Modélisation en Recherche Clinique, Paris, France ; (2) Unité de Biostatistiques et Informatique Médicale, Centre Hospitalo-universitaire Pitié-Salpêtrière, Assistance Publique-Hôpitaux de Paris, Paris, France; (3) Inserm, U974, Paris, France ; (4) Université Pierre-et-Marie-Curie-Paris 6, UMR-S974, CNRS, UMR7215, Institut de Myologie, IFRI4, Paris, France ; (5) Département de génétique Médicale, Hôpital Charles Nicolle, Rouen, France; (6) AP-HP, Groupe Hospitalier Pitié-Salpêtrière, U.F. Cardiogénétique et Myogénétique, Service de Biochimie Métabolique, Paris, France

Contact : sophie.tezenas@psl.aphp.fr

Les gènes modificateurs se définissent comme des gènes modulant l'expression phénotypique d'un autre gène. Lors de la recherche de gènes modificateurs, le phénotype d'intérêt doit être un trait quantifiable de la variabilité clinique chez les personnes porteuses de la mutation du gène majeur. La dystrophie musculaire d'Emery-Dreifuss est une maladie génétique rare, de transmission autosomique (mutations du gène LMNA) ou liée à l'X (mutations des gènes EMD et FHL1) et caractérisée par une double atteinte musculaire squelettique et cardiaque. Il est connu qu'une hétérogénéité phénotypique existe au sein des familles porteuses de mutations dans le gène LMNA, notamment dans la sévérité et l'âge d'apparition de l'atteinte musculaire. Les sujets atteints de la famille EMD1, tous porteurs de la même mutation LMNA p.Q6X, présentent une variabilité clinique : alors que tous les sujets présentent une atteinte cardiaque survenant vers l'âge de 20 ans, certains développent une atteinte musculaire d'âge d'apparition variable. Notre objectif était de rechercher dans la famille EMD1, un ou des gènes modificateurs de l'âge d'apparition du premier trouble de la marche (premier signe d'atteinte musculaire chez les patients) en relation avec la mutation dans le gène majeur. La famille EMD1 comporte 100 individus dont 19 sont porteurs de la mutation LMNA p.Q6X. Cinquante-neuf individus ont été génotypés à l'aide de 291 marqueurs microsatellites répartis uniformément le long du génome. Nous avons utilisé une analyse conjointe de ségrégation et de liaison basée sur des chaînes de Monte Carlo Markov à sauts réversibles implémentée dans le logiciel Loki ${ }^{\circ}$. Nous avons mis en évidence une région de liaison en lien avec l'âge d'apparition du premier trouble de la marche localisé sur le chromosome 2 entre les marqueurs D2S143 et D2S2244 avec un odd ratio de liaison a posteriori, évalué à l'aide d'un indicateur de type « facteur Bayesien * de 36,46 (p-value empirique à 0,00512). Le logarithme du ratio de liaison a posteriori, qui compare la probabilité de liaison a posteriori sur le vrai chromosome à celle sur des chromosomes simulés sous l'hypothèse nulle d'absence de liaison, a été évalué à 3,46. La fréquence de l'allèle mineur (allèle 2) était de $20 \%$. Les individus homozygotes pour l'allèle mineur $(2 / 2)$ présentaient une atteinte musculaire plus précoce de 4 ans (médiane) que les individus homozygotes $1 / 1$ pour l'allèle majeur et qu'une partie des individus hétérozygotes (effet récessif de l'allèle mineur). Par ailleurs, une partie des individus hétérozygotes présentaient une atteinte musculaire très précoce (médiane : 38 ans plus tôt que les homozygotes $1 / 1)$, traduisant un effet d'overdominance de l'allèle modificateur. Actuellement, des analyses moléculaires sont en cours afin d'identifier le gène modificateur se trouvant dans la zone de liaison et afin d'élucider les mécanismes moléculaires par lesquels ils influeraient l'âge d'apparition de l'atteinte musculaire.

Mots-clés : analyse de ségrégation-liaison, dystrophie musculaire, gène modificateur.

D412. MALADIE DE DANON : IMPORTANTE VARIABILITÉ PHENOTYPIQUE AU SEIN D'UNE FAMILLE PORTEUSE D'UNE NOUVELLE MUTATION DU GENE LAMP2

S.-L. Cottinet (1), A.M. Bergemer-Fouquet (2), A. Toutain (3), H. Blasco (4), F. Sabourdy (5), Z. Maakaroun-Vermesse (1), T. Levade (5), A. Chantepie (6), F. Labarthe $(1,7)$

(I) Service de Pédiatrie R, CHU Clocheville, Tours, France ; (2) Service d'Anatomie Pathologique, CHU Bretonneau, Tours, France; (3) Service de Génétique, CHU Bretonneau, Tours, France ; (4) Service de Biochimie, CHU Bretonneau, Tours, France ; (5) Laboratoire de Biochimie Métabolique et INSERM U858, CHU Purpan, Toulouse, France : (6) Service de Pédiatrie A et Cardiologie Pédiatrique, CHU Clocheville, Tours, France ; (7) INSERM U921, CHU Bretonneau, Tours, France
La maladie de Danon est une maladie de surcharge lysosomale rare, liée à l'X, due à des mutations du gène codant pour la protéine LAMP2 (Lysosomal-Associated Membrane Protein-2), et associant myopathie squelettique vacuolaire, myocardiopathie hypertrophique $(\mathrm{MCH})$, et retard intellectuel. Nous rapportons une famille comportant 3 frères et demi-frères et leur mère atteints de façon variable de maladie de Danon en rapport avec une nouvelle mutation. Les trois garçons avaient une myocardiopathie hypertrophique s'accompagnant de troubles de conduction mais de révélation et sévérité variables, se traduisant par un décès par troubles du rythme chez l'aîné, une insuffisance cardiaque à 5 ans chez le plus jeune et l'absence de symptômes cliniques à 15 ans chez le troisième. Ils avaient des myalgies et une faiblesse musculaire variables avec des CK et des enzymes hépatiques élevées à 2 à 3 fois la normale, Les trois avaient un retard intellectuel léger ou modéré. La mère, à 40 ans, avait une atteinte limitée à une myocardiopathie hypertrophique infraclinique avec des contractions ventriculaires prématurées. La biopsie musculaire du cas index avec étude morphologique et immunohistochimie de la protéine LAMP2 a montré des fibres atrophiques avec des vacuoles intracytoplasmiques de surcharge glycogénique et l'absence de protéine LAMP2. L'étude moléculaire du gène LAMP2 a identifié une mutation d'épissage (IVS6+1delG) aboutissant à un skipping de l'exon 6, et ségrégeant avec la maladie dans l'ensemble de la famille. Cette famille porteuse d'une nouvelle mutation du gène LAMP2 illustre la variabilité phénotypique intrafamiliale de la maladie de Danon, et l'intérêt de l'immunomarquage pour le diagnostic de cette affection.

Mots-clés : maladie de Danon, LAMP2, variabilité phénotypique.

\section{IP413. PUCES À FAÇON CGH POUR LA DÉTECTION DE RÉAR- RANGEMENTS INTRAGÉNIQUES : APPLICATION AUX GËNES DES ALPHA-DYSTROGLYCANOPATHIES}

C. Bouchet (I), S. Vuillaumier-Barrot (1), A. Eude-Claye (1), E. Charluteau (1), C. Besson (1), S. Quentin (2), L. Devisme (3), C. Lebizec (1), M. Chelbi (1), P. Landrieu (4), A. Goldenberg (5), K. Maincent (6), P. Loget (7), O. Boute (8), B. Gilbert-Dussardier (9), F. Razavi (10), M. Gonzales (11), N. Seta (1)

(I) APHP, Hôpital Bichat-Claude Bernard, Biochimie Métabolique, France; (2) APHP, Hôpital Saint-Louis, Plate-forme Génomique IUH, France: (3) CHR-U Lille, Anatomie Pathologique, Lille, France; (4) APHP, Hôpital de Bicêtre, Neuropédiatrie, France ; (5) CHU de Rouen, Génétique Médicale, France ; (6) APHP, Hôpital Trousseau, Neuropédiatrie, France; (7) CHG, Anatomie Pathologique, Le Mans, France; (8) CHR-U Lille, Génétique médicale, Lille, France: (9) CHU La miletrie, Génétique médicale, Poitiers, France; (10) APHP Necker, Embryologie, France; (11) APHP, Hôpital Armand-Trousseau, Génétique et Embryologie Médicale, France

Contact : celine.bouchet@bch.aphp.fr

Les alpha-dystroglycanopathies (alpha-DGpathies) sont des dystrophies musculaires de phénotypes très variés à transmission autosomique récessive, caractérisées par une anomalie de glycosylation de l'alpha-dystroglycan, protéine centrale du complexe dystrophine au niveau musculaire et neuronal. Les patients présentent une clinique de dystrophie musculaire congénitale (DMC) avec ou sans retard mental, ou de maladie des ceintures (LGMD) avec une apparition plus tardive des symptômes. Les cas les plus sévères (syndromes de Muscle-Eye-Brain Disease-MEBD et de WalkerWarburg) sont caractérisés par une lissencéphalie de type II (LISII). Ces derniers sont classiquement diagnostiqués en anténatal par échographie, suite à la découverte de l'hydrocéphalie associée à la LISII. Au moins 6 gènes différents: POMT1, POMT2, POMGNT1, FKTN, FKRP et LARGE sont impliqués, sans relation simple avec le phénotype clinique. Dans ces syndromes géniques hétérogènes, le type de mutations est à l'origine de la sévérité clinique. Le diagnostic moléculaire de ces pathologies est donc long et coûteux. De plus, ces gènes sont sensibles à des remaniements intragéniques qui, notamment à l'état hétérozygote, ne sont pas révélés par le séquençage classique sur $\mathrm{ADNg}$. Seuls le séquençage du cDNA ou l'utilisation de PCR quantitative sur chacun des exons étaient jusqu'alors mis en cuvre. Nous avons configuré une puce CGH (Agilent $8 \times 15 \mathrm{~K})$ dirigée contre ces 6 gènes, pour étudier ce type de réarrangement pour les cas d'alpha-DGpathies sans mutation identifiée par séquençage classique. Nous avons ainsi identifié, chez 3 fotus LISII, issus de deux familles distinctes, des réarrangements intragéniques du gène LARGE (3 grandes délétions emportant respectivement 45,105 et $122 \mathrm{~Kb}$ et une duplication des exons 2 à 4 ) et, chez 2 patients MEBD, la même délétion des 4 derniers exons de POMGNT1. Ce sont les premiers cas décrits de remaniements du gène POMGNT1. De plus, nous avons confirmé la présence de deux délétions intragéniques du gène POMT2, préalablement identifiée par l'étude du cDNA, chez 2 patients DMC. Des remaniements géniques ont maintenant été décrits dans trois gènes sur les 6 impliqués dans les 
alpha-Dgpathies. Les puces CGH à façon sont donc très adaptées aux gènes des alpha-DGpathies: elles permettent de compléter le séquençage des différents gènes sur $\mathrm{ADNg}$ par la recherche de grands remaniements intragéniques simultanément sur l'ensemble des gènes, avec un gain certain en terme de coût et de temps.

Mots-clés : alpha-dystroglycanopathies, dystrophie musculaire congénitale, CGH array.

P414. MYOTONIE DE STEINERT : CAS DE DEUX FAMILLES PRÉSENTANT UNE EXPANSION DE TRIPLETS CTG NON AMPLIFIABLE PAR TP-PCR

V. Roth (1), C. Philippe (1), S. Pere (1), F. Mourot (1), P. Kaminsky (2), S. Louis (3), A. Trombini (4), P. Jonveaux (1)

(1) Laboratoire de Génétique Médicale, EA 4368, CHU de Nancy, Vandauvre-lès-Nancy, France; (2) Service de médecine interne Maladies Orphelines et Systémiques, CHU de Nancy, Vandauvre-lès-Nancy, France: (3) Service de Neurologie, Hôpital Central, CHU de Nancy, France; (4) Montois la Montagne, France

Contact : u.roth@chu-nancy.fr

La maladie de Steinert est une affection d'hérédité autosomique dominante, dont l'anomalie moléculaire correspond à une amplification d'une répétition du trinucléotide CTG, située dans la région 3' non codante du gène DM1. Le diagnostic moléculaire repose sur la détermination du nombre de triplets CTG au locus DMI. Dans les cas où la première étape de PCR classique n'a pas permis de mettre en évidence deux allèles de taille normale, une seconde analyse par TP-PCR (pour Triplet Repeat Primed PCR1.) est alors réalisée. Deux types de profils peuvent alors être obtenus : un profil « dentelé » révélateur de la présence d'un allèle pathologique et au contraire un profil « plat », caractéristique dans la majorité des cas de l'absence de grande expansion et donc d'une homozygotie pour l'allèle caractérisé par PCR classique. Cependant, des cas exceptionnels de non amplification de l'allèle muté par TP-PCR ont été décrits, rendant alors indispensable une vérification par Southern Blot de toute mise en évidence de profil plat en TP-PCR. Nous avons étudié deux familles pour lesquelles nous avons mis en évidence une amplification de triplets CTG par la technique de Southern Blot alors que les profils obtenus suite aux réactions de TP-PCR étaient soit plats (identiques à ceux observés chez un patient homozygote normal) soit atypiques avec une interruption du profil dentelé sur une cinquantaine de bases sur électrophorégramme. Nous présentons les profils de TP-PCR obtenus dans les deux familles ainsi que la description clinique des différents individus porteurs d'une répétition de triplets CTG non amplifiable par TP-PCR L'atteinte clinique de l'ensemble des patients que nous avons caractérisé comme porteurs d'une expansion de triplets CTG non amplifiable par TP-PCR semble modérée puisque ceux-ci ne présentaient que des signes discrets voire étaient asymptomatiques. Le séquençage de la région amplifiée permettrait d'identifier si une réelle interruption des répétitions de triplets pourrait être à l'origine de ce phénomène.

Mots-clés : Steinert, TP-PCR, amplification.

IP415. AMYOTROPHIES SPINALES PROXIMALES : CORRÉLATION PHÉNOTYPE GÉNOTYPE

K. Sifi $(1,2)$, Y. Sifi $(2,3)$, N. Abadi $(1,2)$, A. Hamri $(2,3)$, C. Benlatreche $(1,2)$

(1) Laboratoire de biochimie, CHU Benbadis, Constantine, Algérie ; (2) Laboratoire de recherche de biologie et de génétique moléculaire, Faculté de Médecine université de Constantine, Algérie ; (3) Service de neurologie $\mathrm{CHU}$ benbadis, Constantine, Algérie

Contact : sifikarima@yahoo.fr

Introduction: Les amyotrophies spinales ou SMA,constituent un groupe de maladies neuromusculaires le plus souvent de transmission autosomique récessive. Cliniquement il s'agit d'affections très hétérogènes définies par l'âge de début allant de la période anténatale (type I) à l'âge adulte (type IV). Depuis son identification, le gène SMN est reconnu comme le gêne responsable du phénotype SMA.Il est localisé dans la région 5q11-2, $5 q 13-3$, qui contient quatre gènes dupliqués en miroir : le gène SMN(survival motor neuron), présent en deux copies hautement similaires (la copie télomérique ou $\mathrm{SMNt}$ et la copie centromerique ou (SMNc), le gène NAIP(Neuronal apoptosis inhibitory protein), le gène P44 et le gène H4F5. OBJECTIFS : L'objectif de notre travail était de rapporter les caracterisques cliniques et génétiques des patients de phénotype SMA observées dans l'Est Algerien. PATIENTS ET MÉTHODE : Notre étude est du type descriptif retro et prospective réalisée au service de neurologie et laboratoire de biochimie du CHU de Constantine de Janvier 1998 à Juillet 2008. Elle a porté sur 92 patients appartenant à 57 familles répondant tous aux critères définis par le consortium international. L'analyse des délétions des exons 7 et 8 du gène SMN,des exons 4 et 5 du gêne NAIP a été effectuée à Constantine comme décrit par Lefebvre et al. Résultats : 20 de nos patients étaient de type I [ $21,7 \%], 16$ de type II [ $17,39 \%], 53$ de type III $[57,60 \%]$ et 03 de type IV $[3,26 \%]$. Nous avons noté une variabilité phénotypique intrafamiliale dans deux familles. Les délétions homozygotes des exons 7,8 ou 7 et 8 du gène SMNt étaient observées chez 38/57 familles $(66,66 \%)$ avec respectivement $71,42 \%$ dans le type I, $70 \%$ dans le type II, $67,74 \%$ dans le type III et $50 \%$ dans le type IV. L'analyse du gène NAIP a mis en évidence des délétions homozygotes des exons 4 et 5 chez 15 familles $(26,31 \%)$ avec la répartition suivante : $4 / 14$ patients de type I $(28,57 \%), 2 / 10$ patients de type II $(20 \%)$ et $9 / 31$ familles de type III $(15,78 \%)$. DISCUSSION : Nos résultats concordaient avec les données de la littérature et l'analyse clinique de l'ensemble de nos patients était classique en dehors de 6 patients de type $I$ avec survie prolongée au delà de 6 ans sans recours à une ventilation assistée. La variabilité phénotypique intrafamiliale était observée dans dans deux familles. la fréquence globale de délétion du gène SMN semblait basse par rapport à celle observée dans de nombreuses études (Chang et al. ; Lefebvre et al. ; Brahe et al.), mais prochent de ceux rapportés par C.A. Kim et al., Thompson et al. et R.M. Shawky et al. CONCLUSION : Il nous semble pertinent de prendre en compte considérablement des familles sans délétions dans le gène SMNt, ces patients pourraient porter d'autres délétions dans d'autres exons ou des mutations ponctuelles,comme il pourrait s'agir d'une hétérogénéité génétique.

\section{Mots-clés : SMA, SMN, NAIP.}

\section{UP416. MYOPATHIES CENTRONUCLÉAIRES : STRATÉGIES DIAGNOSTIQUES ET BASES UMD SPÉCIFIQUES MTM1, DNM2 ET BIN1}

E. Schaefer (1, 2), M. Bitoun (3), D. Hamroun (4), V. Humbertclaude (4), C. Beroud (4), J.L. Mandel (1, 5), J. Laporte (5), V. Biancalana $(1,2)$ (1) Laboratoire Diagnostic Génétique, Faculté de Médecine - CHRU, Strasbourg, France; (2) EA3949, Faculté de Médecine - CHRU, Strasbourg, France : (3) INSERM U974 - Institut de Myologie - Groupe Hospitalier Pitié-Salpêtrière, Paris, France: (4) Laboratoire de Génétique Moléculaire et INSERM U827, Montpellier, France ; (5) IGBMC, Département de Neurobiologie et Génétique, Illkirch, France

Contact : valerie.biancalana@chru-strasbourg.fr

Les myopathies (myotubulaires) centronucléaires (CNM) sont caractérisées par une faiblesse musculaire associée à la présence de noyaux centrés à la biopsie sans régénération excessive. Trois formes à mode de transmission différents ont été décrites. La forme récessive liée à au chromosome $\mathrm{X}$ est usuellement une forme néonatale sévère due à des mutations du gène MTM1. La forme autosomique dominante, généralement peu sévère et à début tardif, est liée à des mutations du gène Dynamine 2 (DNM2). Étonnamment, DNM2 est également impliqué dans une forme de neuropathie de Charcot-Marie-Tooth autosomique dominante (CMT type 2 et intermédiaire). Récemment, des mutations dans le gène amphiphysin 2 (BIN1) ont été identifiées dans 3 familles consanguines présentant une forme modérée autosomique récessive débutant à la naissance ou dans l'enfance. Nos laboratoires sont les seuls laboratoires en France à être impliqués dans l'étude moléculaire de ces 3 gènes au titre de la recherche ou du diagnostic, dans le cadre des myopathies centronucléaires. De cette expérience et des données de la littérature, nous proposons une stratégie de diagnostic moléculaire basée sur plusieurs points : 1/ le mode de transmission $2 /$ les données histopathologiques $3 /$ les données cliniques et notamment les formes de sévérité ou de début atypiques associées à des mutations particulières 4/ le spectre mutationnel de MTM1, DNM2 et BIN1. Enfin, nos laboratoires sont promoteurs de projets de bases UMD internationales pour les 3 gènes afin de faciliter la recherche d'informations sur les aspects épidémiologiques, le spectre des mutations, la corrélation génotype-phénotype et l'histoire naturelle de la maladie. Ces données serviront deux objectifs majeurs. L'un des objectifs est l'amélioration du diagnostic moléculaire en partie pour affiner l'arbre décisionnel de l'étude des 3 gènes, mais aussi pour permettre une meilleure prédiction de l'effet pathologique de variants rarissimes, notamment dans le gène MTM1. En effet, ces bases compileront les études génomiques, ARN et protéine dispersées au niveau international, et proposeront des outils UMD de prédiction d'effet pathologique notamment pour les mutations d'épissage et les mutations faux sens. L'autre objectif de ces bases est de faciliter les études de faisabilité de diverses approches thérapeutiques et le recrutement des patients dans des essais cliniques. À cette fin, les 3 bases partageront un tronc commun de données cliniques utilisant des items harmonisés avec ceux d'autres bases UMD et TREAT-NMD de myopathie congénitale. En conclusion, dans le cadre d'un projet d'amélioration du diagnostic et de la 
prise en charge pour les patients atteints de myopathies centronucléaires, nous présentons des arbres décisionnels pour faciliter le diagnostic moléculaire, ainsi que les objectifs et l'état d'avancement de bases UMD pour les gènes MTM1, DNM2 et BIN1.

Mots-clés : myopathie centronucléaire, stratégie diagnostique, base UMD.

P417. DYSTROPHINOPATHIES ET PROFIL D'INACTIVATION DE L'X : À PROPOS DE 24 CONDUCTRICES SYMPTOMATIQUES À L'ÂGE PÉDIATRIQUE

S. Mercier (1), A. Toussaint (2), C. Beugnet (2), C. De Barace (3), A. Toutain (4), M. Raynaud (4), P. Marcorelles (5), Leila Lazaro (1), L. Pasquier (1), R. Ben Yaou (2), F. Leturcq (2), J. Chelly (6), I. Desguerre (6, 7) (1) Service de Génétique Clinique, CHU Hôpital Sud, UMR 6061 CNRS, Université Rennes I, Rennes, France : (2) Laboratoire de Génétique et de Biochimie Moléculaire, Hôpital Cochin, APHP, Paris, France ; (3) Service de Pédiatrie, CH de Saint-Brieuc, France ; (4) Service de Génétique Médicale, CHU Hôpital Bretonneau, Université de Tours, Tours, France; (5) Service d'Anatomie Pathologique, Hôpital Morvan, Brest, France; (6) Département de Génétique et Développement, U567, UMR8104, Institut Cochin, Paris, France; (7) Service de Neuropédiatrie, Hôpital NeckerEnfants Malades, APHP, Paris, France

Contact : sandra.mercier@chu-rennes.fr

Les dystrophies musculaires de Duchenne (DMD) et de Becker (DMB) [MIM 310200] sont des maladies liées à l'X causées par des mutations dans le gène de la dystrophine. La fréquence des conductrices symptomatiques est estimée entre $3 \%$ et $22 \%$ de l'ensemble des conductrices. Nous rapportons la plus grande série décrite à ce jour à propos de 24 conductrices symptomatiques à l'âge pédiatrique dont le statut a été confirmé par l'étude moléculaire du gène DMD. Dans $30 \%$ des cas, l'atteinte musculaire s'est manifestée initialement par une intolérance à l'effort voire une forme pseudométabolique avec des épisodes de rhabdomyolyse. Globalement, la sévérité de l'atteinte musculaire squelettique est corrélée à l'âge de début des symptômes. Une atteinte cardiaque à type de cardiomyopathie dilatée a été mise en évidence chez $25 \%$ des conductrices âgées de plus de 30 ans. Nous mettons l'accent sur l'atteinte cognitive à type de déficience mentale ou difficultés d'apprentissage qui a été constatée dans près de $30 \%$ des cas. Une élévation du taux de CPK était présente dans $100 \%$ des cas. Un aspect dystrophique de la biopsie musculaire, un marquage spécifique en mosaïque de la dystrophine lié à l'inactivation de l'X, ainsi qu'une diminution de la quantité de dystrophine sur le Western-Blot ont été retrouvées dans plus de $80 \%$ des cas. L'inactivation de l'X joue un rôle fondamental dans la symptomatologie des conductrices comme cela a été démontré précédemment, mais l'interprétation des profils d'inactivation de l'X sur le sang et/ou sur le muscle reste très délicate. En effet, dans notre étude, nous avons observé quasiment la même proportion de profils aléatoires que de biais significatifs d'inactivation de l'X. À l'échelle individuelle, il n'est pas possible d'établir un pronostic fonctionnel basé sur le profil d'inactivation de l'X. Des facteurs modificateurs encore indéterminés, environnementaux ou génétiques, participent très probablement à cette variabilité phénotypique ce qui doit amener à une certaine prudence pour le conseil génétique.

Mots-clés : DMD, conductrices symptomatiques, inactivation de l'X

\section{EP418. L'IMPORTANCE DE LA CONVERSION GÉNIQUE ET D'AUTRES REARRANGEMENTS DE NOVO DU GENNE SMN1 DANS LE DIAGNOSTIC MOLÉCULAIRE DE L'AMYOTROPHIE SPINALE}

Z. Elhaffaf (1), B. Maranda (2), L. Simard (3), R. Gagné (2), S. Bélanger (4), G. Mitchell (1), Li Fan (1)

(1) Service de Génétique médicale et Laboratoire du Diagnostic Moléculaire, CHU Sainte-Justine, Université de Montréal, Montréal, QC, Canada; (2) Service de Génétique médicale, CHUL, Québec, QC, Canada ; (3) Department of Biochem and Med Genet, University of Manitoba, Winnipeg, Manitoba, Canada; (4) Service de Neurologie, CHU Sainte-Justine, Université de Montréal, Montréal, QC, Canada

Contact : zelhaffaf@sympatico.ca

INTRODUCTION : L'amyotrophie spinale (AMS 5q), maladie autosomique récessive ayant un taux de porteur d'environ $1 / 40$, est causée dans $95 \%$ des cas par l'absence de l'exon 7 du gène SMN1 sur le chromosome $5 q 12-13$. Un gène paralogue voisin et quasi identique, SMN2, differre de SMN1 par la présence d'une mutation synonyme $(840 \mathrm{C}>\mathrm{T})$ qui empêche l'épissage normal. La région SMN1/SMN2 est prédisposée à des événements de conversion génique et de duplication/duplication. La détermination du nombre de copies total chez un individu n'est pas discriminative entre une position en cis ou en trans des copies SMN1 ou SMN2. Donc on peut avoir recours à l'analyse familiale de liaison afin de déterminer le statut des individus à risque d'être hétérozygote pour un allèle délété en SMN1. Nous décrivons deux exemples où une conversion génique a été démontré par la comparaison de données haplotypiques et par l'analyse du nombre de copie du gène SMN1. MATÉRIEL ET MÉTHODES La méthodologie diagnostique de quantification a évolué d'une analyse de PCR quantitative dans laquelle la distinction entre SMN1 et SMN2 dépendait d'une digestion de restriction (Famille A), à une analyse par RT-PCR quantitative avec des amplifications spécifiques de SMN1 et de SMN2 (Famille B). L'analyse des haplotypes dans la région de SMN1/2 a été motivé par différentes raisons diagnostiques dans deux familles non consanguines, l'une d'origine moyen orientale (Famille A, diagnostic prénatal) et l'autre, canadienne-française (Famille B, analyse pour conseil génétique). RÉSULTATS Dans la famille A, dans les amniocytes on retrouvait chez le fotus le même haplotype que celui d'une sœur atteinte. Toutefois, une copie du SMN1 était présente, confirmée par différentes techniques et suggérant un état d'hétérozygote. La grossesse a été poursuivie et l'enfant est maintenant en bonne santé. Dans la famille B, l'analyse du nombre de copie ne permettaient pas de déterminer le statut d'un frère non-atteint du probant. L'analyse haplotypique des parents et de la fratrie a révélé un haplotype identique chez le probant (SMN1:SMN2, 0:3) et chez un autre frère non-atteint (SMN1:SMN2, 1:2). CONCLUSION La conversion génique doit être considérée dans l'amyotrophie spinale 5q. Elle peut être critique pour le diagnostic moléculaire. Une discordance entre l'analyse haplotypique et l'analyse du nombre de copie SMN1 peut témoigner d'un phénomène de conversion génique ou d'un autre réarrangement génomique spontanée dans cette région. Pour cette raison, l'analyse diagnostique de l'AMS 5q par liaison doit être interprétée avec précaution.

Mots-clés: diagnostic moleculaire, amyotrophie spinale, conversion génique.

aP419. MUTATIONS DANS LE GENE LPIN1: UNE CAUSE MAJEURE DE RHABDOMYOLYSE SÉVÈRE DU JEUNE ENFANT C. Michot (1), L. Hubert (1), M. Brivet (2), M. Jeanpierre (3), L. De Meirleir (4), R. Horvath (5, 6), P. Chinnery (6), A. Munnich (1), O. Elpeleg (7), Y. De Keyzer (1), P. De Lonlay (1)

(l) INSERM U-781, Université Paris-Descartes et Centre de Référence des Maladies Métaboliques, Paris, France: (2) Service de Biochimie Hôpital du Kremlin-Bicêtre, France ; (3) Département de Biochimie, Université Paris Descartes, Paris, France; (4) Neuropédiatrie - Maladies Métaboliques, Bruxelles, Belgique ; (5) Friedrich-Baur-Institut, Université Ludwig-Maximilians, Munich, Allemagne; (6) Mitochondrial Research Group, Institute for Aging and Health, Université de Newcastle, Newcastle upon Tyne, UK ; (7) Département de Génétique et Maladies Métaboliques, Hadassah - Hebrew University Medical Center, Jerusalem, Israel Contact : pascale.delonlay@nck.aphp.fr

Les rhabdomyolyses récurrentes de l'enfant correspondent à la destruction de fibres musculaires striées squelettiques, dont la principale cause est un défaut d'oxydation des acides gras (OAG). La moitié des cas restent cependant sans cause identifiée. Récemment, le gène lipine 1 (LPIN1) a été impliqué comme une nouvelle étiologie de rhabdomyolyse, de transmission récessive autosomique. Nous avons étudié le gène LPIN1 dans une cohorte de 24 patients ayant présenté, depuis la petite enfance, un ou plusieurs épisodes de rhabdomyolyse sévère (créatine phosphokinase $=\mathrm{CPK}>10000 \mathrm{UI} / \mathrm{L}$ ), et pour lesquels un déficit de l'OAG, une glycogénose et une dystrophinopathie ont été éliminés. Les 24 patients (17 Eurocaucasiens, 6 Africains et 1 Asiatique) ont un examen clinique normal et un taux de CPK normal ou subnormal en dehors des accès, excepté un patient présentant un déficit musculaire modéré. Deux mutations du gène LPIN1 ont été identifiées chez $50 \%$ de nos patients. Aucune mutation de novo n'a été observée. $7 / 12$ patients mutés, tous Caucasiens, sont porteurs, à l'état homozygote $(2 / 7)$ ou hétérozygote composite $(5 / 7)$, d'une délétion récurrente emportant les exons 18 et 19 sur ADN complémentaire. L'étude de l'ADN génomique par PCR long range a confirmé l'existence d'un allèle plus court par rapport aux contrôles. Les 7 patients porteurs de la délétion présentent les mêmes points de cassure (du nucléotide +3371 de l'intron 17 au nucléotide +779 de l'intron 18 , à 33 nucléotides en 5 ' de l'exon 19) et possèdent un haplotype commun de marqueurs microsatellites dans une petite région entourant la délétion. Ces résultats sont évocateurs d'un effet fondateur ancien. La fréquence de $50 \%$ de mutations dans notre cohorte et l'existence d'une délétion récurrente de diagnostic rapide (PCR long range) incite à rediscuter la hiérarchie des investigations à réaliser devant les rhabdomyolyses sévères du petit enfant. Nous proposons que l'étude de LPIN1 soit réalisée après l'exclusion d'un déficit de I'OAG, mais avant toute biopsie musculaire, qui reste un acte invasif. Mots-clés : rhabdomyolyse, lipine 1, effet fondateur. 
DP20. ASPECTS CLINIQUES ET MOLÉCULAIRES AU COURS DE L'AMYOTROPHIE SPINALE INFANTILE : ÉTUDE DE 12 PATIENTS DU SUD TUNISIEN

N. Belguith (1, 2), H. Kamoun (1, 2), B. Ben Rhouma (1), I. Chabchoub (3), F. Kamoun (4), R. Keskes (1), C.H. Triki (4), M. Hachicha (3), F. Fakhfakh (1)

(1) Laboratoire de Génétique Moléculaire Humaine, Faculté de Médecine de Sfax, Tunisie ; (2) Service de Génétique Médicale, Hôpital Hédi Chaker, Sfax, Tunisie; (3) Service de Pédiatrie Hôpital Hédi Chaker, Sfax, Tunisie ; (4) Service de Neurologie de l'enfant et de l'adolescent, Hôpital Hédi Chaker, Sfax, Tunisie

Contact : hassen.kamoun@rns.tn

L'amyotrophie spinale (AMS) est une maladie neuromusculaire, à transmission autosomique récessive. Elle est due à la dégénérescence du motoneurone de la corne antérieure de la moelle épinière. Le type I ou maladie de Werdnig-Hoffman est la forme fatale débutant avant l'âge de six mois. Le gène SMN responsable de l'AMS est localisé sur le chromosome 5 en $5 q 12$-q13, dans une région dupliquée. $95 \%$ des anomalies génétiques de l'AMS sont dues à la délétion des exons 7 et/ou 8 de sa copie télomérique (SMN1). Nous rapportons 12 patients présentant une hypotonie, un retard ou une régression des acquisitions psychomotrices et ayant à l'examen une abolition des réflexes. L'âge de début variant de 1 à 6 mois. Les données éléctromyographiques en faveur d'une atteinte neurogène ont fait suspecter l'AMS type I. Un seul patient est encore vivant et âgé de 2 ans et demi ; il présente un retard psychomoteur sans troubles respiratoires. Les autres patients sont décédés dans un tableau d'insuffisance respiratoire au bout de quelques mois. Après extraction de l'ADN génomique à partir des prélèvements sanguins, une amplification par PCR moyennant des amorces flanquants les exons 7 et 8 du gène SMN a été réalisée suivie d'une digestion enzymatique via les enzymes de restriction DraI et DdeI respectivement. Nos résultats ont confirmé la délétion des deux exons 7 et 8 de la copie télomérique chez 10 patients et de l'exon 7 uniquement chez les deux autres. Ces données offrent ainsi un grand intérêt pour le diagnostic, soulignant le rôle important de l'électromyogramme, et permettant de donner un conseil génétique et de proposer à temps un diagnostic prénatal. D'autre part nous constatons l'absence de corrélation génotype phénotype déjà rapportée dans la littérature. De plus nous confortons l'idée que la délétion de l'exon 7 à elle seule peut engendrer le phénotype grave. Par ailleurs le phénotype modéré que présente l'un de nos patients, présentant la délétion des deux exons, peut s'expliquer par un nombre de copies SMN2 élevé pouvant jouer un rôle dans la modération de l'expression clinique ; hypothèse à vérifier en perspectives.

Mots-clés : amyotrophie spinale infantile, gène SMN, corrélation génotype phénotype.

IP421. DOMINANT AND RECESSIVE MUTATIONS CAUSING EARLY ONSET COLLAGEN VI-RELATED MUSCLE DISOR. DERS WITH VARIABLE SEVERITY: CORRELATION WITH TRANSCRIPT STABILITY AND CLINICAL PHENOTYPE

L. Briñas (1), P. Richard $(2,3)$, S. Quijano-Roy $(1,4)$, C. Gartioux (1), C. Ledeuil (2, 3), S. Makri (5), A. Ferreiro (4, 6, 7), S. Maugenre (8), H. Topaloglu (9), G. Haliloglu (9), I. Penisson-Besnier (10), P.-Y. Jeannet (11), L. Merlini (12, 13), C. Navarro (14), A. Toutain (15), D. Chaigne (16), I. Desguerre (17, 18), C. de Die-Smulders (19), M. Dunand (20), B. Echenne (21), B. Eymard (7), T. Kuntzer (20), K. Maincent (22), M. Mayer (22), G. Plessis (23), F. Rivier (21), F. Roelens (24), T. Stojkovic (1, 7), A.L. Taratuto (25), F. Lubieniecki (26), S. Monges (27), C. Tranchant (28), L. Viollet $(4,29)$, N.B. Romero (1), B. Estournet (4), P. Guicheney (8), V. Allamand (1)

(1) Inserm U974-UPMC Paris 6 UMR_S974-CNRS UMR7215-Institut de Myologie, Groupe Hospitalier Pitié-Salpêtrière, Paris, France ; (2) Inserm US956, CHU Pitié-Salpêtrière, Paris, France; (3) AP-HP, Groupe Hospitalier Pitié-Salpêtrière, UF Cardiogénétique et Myogénétique, Service de Biochimie Métabolique, Paris, France ; (4) AP-HP, Service de Pédiatrie, Centre de Référence Maladies Neuromusculaires (GNMH) Hôpital Raymond-Poincaré, Garches, France ; (5) Service de Neurologie. Établissement Hospitalier Spécialisé Ali Ait Idir, Alger, Algeria; (6) Inserm U787-Institut de Myologie, Groupe Hospitalier Pitié-Salpêtrière, Paris, France; (7) Centre de Référence Neuromusculaire Paris-Est, Institut de Myologie, Groupe Hospitalier Pitié-Salpêtrière, Paris, France : (8) Inserm US956, CHU Pitié-Salpêtrière, Paris, France; (9) Section of Neurology, Department of Paediatrics, Hacettepe Children's Hospital, Ankara, Turkey; (10) Centre de Référence Maladies Neuromusculaires, Département de Neurologie, Centre Hospitalier Universitaire, Angers, France; (1I) Unité de Neuropédiatrie, CHUV - BHII, Lausanne, Switzerland;
(12) Laboratorio di Miologia, Servizio di Genetica Medica Ospedale S. Anna, Ferrara, Italy; (13) Laboratorio di Biologia, IOR-IRCCS, Bologna, Italy: (14) Servicio de Anatomía Patológica, Hospital Meixoeiro, Complexo Hospitalario Universitario de Vigo (CHUVI), Vigo, Spain; (15) Service de Génétique, Centre Hospitalier Universitaire Bretonneau, Tours, France; (16) Clinique Sainte-Odile, Strasbourg, France: (17) APHP, Département de Neuropédiatrie, Centre de référence des maladies neuromusculaires "Garches-Necker-Mondor-Hendave », Hôpital NeckerEnfants Malades, Paris, France; (18) Département de Neurosciences, Équipe 10, Inserm U841, Institut Mondor de recherche biomédicale, Paris XII, Créteil, France; (19) Department of Clinical Genetics, University Hospital Maastricht, Maastricht, The Netherlands: (20) Unité NerfMuscle, Service de Neurologie, BH07/309, Centre Hospitalier Universitaire Vaudois, Lausanne, Switzerland; (2I) CHU Montpellier, Service de Neuropédiatrie-Centre de référence Maladies Neuromusculaires Grand Sud Ouest, Hôpital Gui de Chauliac, Montpellier, France ; (22) Hôpital Armand-Trousseau, Service de Neuropédiatrie, Consultation Maladies Neuromusculaires, Paris, France: (23) Service de génétique médicale, DGR, CHU Clémenceau, Caen Cedex 9, France; (24) Dominiek Savio Instituut Gits, Roeselare, Belgium; (25) Department of Neuropathology/ FLENI, Institute for Neurological Research, Buenos Aires, Argentina ; (26) Department of Pathology, Garrahan National Pediatric Hospital, Buenos Aires, Argentina : (27) Department of Neuropediatrics, Garrahan National Pediatric Hospital, Buenos Aires, Argentina : (28) Service de Neurologie, Hôpitaux Universitaires de Strasbourg, Strasbourg, France ; (29) Inserm U781, Hôpital Necker-Enfants Malades, Paris, France

Contact : l.brinas@institut-myologie.org

Objective : Ullrich Congenital Muscular Dystrophy (UCMD), caused by mutations in the genes encoding collagen VI (COL6), is the archetype of early onset COL6-related myopathies. The great heterogeneity of clinical severity and molecular spectrum complicates the diagnosis and the establishment of genotype-phenotype correlations. Here we extensively characterized, at the clinical, cellular and molecular levels, 49 patients classified according to the severity and progression of their symptoms in order to infer genotype-phenotype correlations. Methods : Patients were classified in three groups : early severe $(18 \%)$, moderate progressive $(53 \%)$ and mild $(29 \%)$. COL6 secretion was analyzed on patient-derived skin fibroblasts. Chain-specific transcript levels were quantified by RT-PCR, and mutation screening was performed by sequencing of cDNA. Results: COL6 secretion was altered in all but one fibroblast cultures studied. We identified 56 mutations, mostly novel, private, and dominant de novo. We showed that mutations causing premature termination codons (PTC) or in-frame insertions strikingly destabilize the corresponding transcripts. Homozygous PTC-causing mutations in the triple helix domains led to the most severe phenotypes (ambulation never achieved). Dominant de novo splice mutations leading to in-frame exon skipping also cause severe clinical phenotypes (loss of ambulation at about 10 years). Interpretation : This work emphasizes the complexity of the diagnosis of early onset COL6-related myopathies, and demonstrates that quantitative RT-PCR is a helpful tool for the identification of the mutation-bearing genes in the case of PTCs or in-frame insertions. Some genotype-phenotype correlations clearly appeared, providing important clues for the diagnosis and management of patients and the development of future clinical trials.

Mots-clés: collagène VI, matrice extracellulaire, corrélation génotype-phénotype.

1P422. DYSTROPHINOPATHIES: UN DÉFAUT PEUT EN CACHER UN AUTRE :

D. Thorel (1), D. Méchin (1), S. Tuffery-Giraud (2, 3), M. Claustres (1, 2, 3), P. Khau Van Kien (1)

(1) Laboratoire de Génétique Moléculaire, CHU Montpellier, Montpellier. France ; (2) INSERM U827, Montpellier, France : (3) Université Montpellier I, Montpellier, France

Contact : p-khau_van_kien@chu-montpellier.fr

Le diagnostic moléculaire des dystrophies musculaires de Duchenne et de Becker (DMD/B) est particulièrement complexe du fait de la grande taille du gène DMD, de la grande diversité des lésions moléculaires et de leurs effets sur l'expression du gène. Le choix des stratégies mises en œuvre tient compte de différents paramètres dont la connaissance approfondie du spectre de mutations, de leurs fréquences et du pedigree (cas sporadique/ familial). Dans les situations de cas apparemment sporadiques, le dosage de créatine phosphokinase (CK) chez les apparentées est utile à l'analyse du pedigree. Nous rapportons ici 5 cas où la connaissance du défaut génétique a été essentielle pour clarifier la situation diagnostique, le statut des différentes apparentées de la famille et formuler un conseil génétique fiable, - Cas $\mathrm{n}^{\circ} 1$ : cas sporadique, décédé de myopathie de Duchenne 
(DMD) sans prélèvement disponible. Dans cette famille, la tante maternelle du cas index présente des CK élevées (trois dosages indépendants et absence de facteur élévateur favorisant connu). Ceci oriente vers une transmission familiale et indique que la mère du cas index est par extension conductrice obligatoire. L'étude d'haplotypes des deux sæurs nous montre qu'elles sont génoidentiques au locus dystrophine. Pourtant une mutation non-sens identifiée chez la mère du cas index n'est pas retrouvée sur le prélèvement de la tante. La cause de l'élévation des CK chez cette apparentée reste indéterminée. - Cas $\mathrm{n}^{\circ} 2$ : survenue d'une dystrophie musculaire congénitale mérosine négative (DMC1A) dans un pedigree présentant deux cas de DMD. La détermination des mutations dans le gène LAMA2 a permis de redresser le diagnostic initial, faussement orienté vers une DMD compte tenu de l'histoire familiale. - Cas $\mathrm{n}^{\circ} 3$ et 4 : survenue dans un même pedigree de deux mutations indépendantes dans le gène DMD (deux familles non apparentées), - Cas n ${ }^{\circ} 5$ : cardiomyopathie mitochondriale de révélation néonatale révélant une DMD chez un nourrisson. Une élévation importante des CK est découverte chez un nourrisson consanguin présentant un ventricule gauche non compacté et une décompensation cardiaque. L'analyse d'une biopsie de muscle objective un déficit de la chaîne respiratoire mitochondriale et un déficit total en dystrophine. L'analyse du gène DMD a permis de mettre en évidence une mutation non-sens héritée de la mère. Toutefois, le conseil génétique ne peut être formulé que pour la DMD dans cette famille dans l'attente du défaut moléculaire responsable de la cardiopathie mitochondriale. Ces données illustrent l'importance de réaliser des analyses exhaustives dans chaque famille; chaque apparentée et chaque cas doivent être génotypés afin de sécuriser le diagnostic, d'aboutir à une prise en charge adaptée des patients et à un conseil génétique fiable.

Mots-clés : dystrophine, pièges diagnostiques, conseil génétique.

\section{GP423. RECHERCHE DE MUTATION DANS LE GÈNE THAP1 (LOCUS DYT6) DANS UNE SÉRIE DE 117 PATIENTS ATTEINTS DE DYSTONIE PRIMAIRE}

F. Clot (1), D. Grabli (2, 3), P. Burbaud (4), P. Damier (5), P. Krystkowiak (6), A. Mendes (7), P. Pollak (8), C. Sân (1), E. Roze (2), M. Vidailhet (2, 3), A. Durr $(1,3)$, A. Brice $(1,3)$

(1) AP-HP, Département de Génétique et Cytogénétique, Groupe Hospitalier Pitié-Salpêtrière, Paris, France ; (2) AP-HP, Département de Neurologie, Groupe Hospitalier Pitié-Salpêtrière, Paris, France ; (3) CRicm INSERM UMRS975, Paris, France; (4) Service de Neurophysiologie Clinique, CHU de Bordeaux, Pessac, France; (5) CHU Nantes, INSERM, CICO004, Nantes, France; (6) Service de Neurologie, CNRS UMR 8160 , CHU d'Amiens, France ; (7) Hospital Geral Santo Antonio, Porto, Portugal ; (8) Service de Neurologie, CHU de Grenoble, France

Contact : fabienne.clot@psl.aphp.fr

La dystonie est une maladie rare, caractérisée par des contractions musculaires involontaires et prolongées. C'est une maladie très hétérogène sur le plan clinique et génétique. Récemment des mutations du gène THAP1, au locus DYT6, codant pour la protéine « thanatos_associated protein », ont été identifiées dans les dystonies primaires de transmission autosomique dominante avec une pénétrance réduite de $60 \%$. À ce jour 12 mutations ont été rapportées dans la littérature. Il s'agit de mutations faux-sens ou de délétions entraînant la prédiction d'un codon stop prématurée de la protéine. Les dystonies décrites sont fréquemment généralisées et débutent au niveau des muscles brachiaux, cervicaux ou céphaliques. La moyenne d'âge de début des premiers symptômes est de 13 ans. Afin de préciser le spectre clinique des patients porteurs de mutations du gène THAP1, nous avons séquencé le gène dans une série de 117 patients dont 22 avec une histoire familiale de dystonie, d'origine caucasienne et exclus pour le gène DYT1. Ces patients présentaient des dystonies généralisées, segmentaires ou focales et avaient débutés leurs premiers symptômes avant 26 ans. Nous avons identifiés 5 patients porteurs de nouvelles mutations. Deux mutations sont des mutations faux sens (p.Ser6Pro et p.Leu72Arg) et 3 sont des délétions entraînant soit la perte d'un acide aminé très conservé (p.Asn69del), soit l'apparition d'un codon stop (p.Ala7GlufsX23 et Arg146AspfsX9). Excepté la mutation Arg146AspfsX9, présente dans le signal de localisation nucléaire, les autres mutations sont localisées dans le domaine de liaison à I'ADN, comme la plupart des mutations identifiées dans la littérature. Parmi ces 5 cas index porteurs d'une mutation du gène THAP1, 3 n'ont pas d'histoire familiale de la maladie et 2 cas sont familiaux. Au total, 6 patients sont porteurs d'une mutation. L'age de début moyen de la maladie est de 14 ans $+/-4,7$ ( 9 à 20 ans) et le premier symptôme le plus fréquemment présent est une dystonie cervicale $(4 / 6)$. Le type de dystonies observées sont soit généralisées (4), soit segmentaires (1), soit focales (1). Les parties du corps les plus touchés sont le cou et/ou
D'autre part, 2 patients sur 6 présentent des myoclonies. Les patients sans mutation ont un âge moyen de début de la maladie similaire aux patients mutés (12,5 vs 14 ans). En revanche, seulement $29 \%$ des patients sans mutation ont débuté leurs premiers symptômes au niveau du cou. Dans notre série de patients, seulement $4 \%$ sont porteurs d'une mutation du gène THAP1. L'expressivité de la maladie est variable, allant d'une dystonie focale à une dystonie généralisée. La dystonie débute dans la partie supérieure du corps (en particulier le cou) chez l'adolescent ou le jeune adulte. Enfin, la recherche de mutations dans les cas apparemment sporadiques ne doit pas être écartée.

Mots-clés : dystonie, DYT6, THAP1.

EP24. RECHERCHE DE DUPLICATION DU GÈNE LAMIN BI DANS UNE COHORTE DE 70 PATIENTS PRÉSENTANT UNE LEUCODYSTROPHIE DE CAUSE INDÉTERMINÉE DE DÉBUT ADULTE (ADLD)

E. Eymard-Pierre (1, 2), P. Labauge (3), P. Vago (1), J.L. Venaise (4), S. Vukusic (5), C. Confavreux (5), O. Dereeper (6), M. Clanet (7), O. Boespflug-Tanguy $(2,8)$

(1) Laboratoire de cytogénétique médicale, Clermont-Ferrand, France; (2) INSERM UMR 931 CNRS 6647 Clermont University, GReD, ClermontFerrand, France ; (3) Service de Neurologie, CHU de Nimes, France ; (4) Service de Neurologie, CHU d'Angoulême, France; (5) Service de Neurologie, CHU de Lyon, France; (6) Service de Neurologie, CHU de Calais, France: (7) Service de Neurologie, CHU de Toulouse, France; (8) Service de Neuropédiatrie, Hôpital Robert-Debré, Paris, France

Contact: e_pierre@chu-clermontferrand.fr

Les maladies génétiques, touchant primitivement et préférentiellement la substance blanche du système nerveux central (SNC) ou leucodystrophies, représentent un groupe très hétérogène de maladies « rares ». Les nombreuses formes sans marqueur biochimique ont bénéficié de l'identification de plus d'une dizaine de gènes mais l'expression phénotypique de ces différentes mutations reste encore mal connue en particulier chez l'adulte. Des duplications contenant une région comprenant le gène lamin B1 (LMNB1), ont été décrites comme étant à l'origine d'une forme de leucodystrophie autosomique dominante démyélinisante de début adulte (LADA) ou ADLD (Adult-onset autosomal dominant leukodystrophy) [Padiath et al., 2006]. Le début se situe classiquement dans la quatrième ou cinquième décade par des signes cérebello-spastique associés à des troubles dysautonomiques (dysurie en particulier). L'existence d'une démyélinisation confluente du SNC peut chez certains patients faire porter le diagnostic de forme chronique progressive de sclérose en plaques (SEP). Nous avons recherché une duplication du gène de la lamin B1 dans une cohorte de 70 patients présentant un phénotype d'ADLD par une technique de PCR quantitative multiplex fluorescente (QMPSF). Cette quantification génique explore les exons 2 et 4 du gène lamin $\mathrm{B} 1$ en parallèle avec deux gènes contrôles l'un situé sur le chromosome $\mathrm{X}$ (contrôle du sexe) et l'autre sur l'exon 4 du gène CFTR (gène pour la normalisation dans le calcul des ratios). Une duplication du gène lamin B1 a été mise en évidence chez 5 patients, 3 étant des formes sporadiques et 2 patients appartenant à la même famille (mère et fils). Deux groupes phénotypiques se dégagent à la fois chez les patients porteurs d'une duplication lamin B1 et ceux sans duplication : un groupe avec un tableau clinique et IRM de leucodystrophie (paraparésie spastique et ataxie d'évolution progressive, atteinte symétrique en IRM de la substance blanche) et un autre groupe avec un tableau clinique, biologique et IRM de pseudo SEP. Au total, les ADLD représente un groupe clinique et génétique très hétérogène. Les duplications du gène lamin B1 y sont rarement retrouvés (environ $7 \%$ des cas). Les patients dupliqués n'ont pas de spécificité phénotypique permettant de les individualiser des patients non dupliqués.

Mots-clés : leucodystrophie, duplication, lamin B1.

\section{EP425. SYNDROME DE MOHR-TRANEBJAERG DANS UNE FAMILLE ANTILLAISE}

M. Lackmy Port-Lis (1), H. Bataille (2), D. Bouamama (2), A. Verloes (3) (1) Unité de Génétique Médicale, CHU Pointe à Pitre-Abymes, Guadeloupe: (2) Service de Pédiatrie, CHU Pointe à Pitre-Abymes, Guadeloupe; (3) Département de Génétique, APHP-Hôpital Robert DEBRÉ, Paris, France

Contact : alain.verloes@rdb.aphp.fr

Le syndrome de Mohr-Tranebjaerg (MTS) ou Deafness-Dystonia Syndrome (DDS) est une maladie neurodégénérative récessive liée à l'X, débutant par une surdité neurosensorielle apparaissant dans l'enfance, généralement avant 10 ans. Elle se complique progressivement de dystonie, de troubles du comportement, de troubles visuels et d'une régression ou d'un 
retard mental. L'évocation du diagnostic de MTS chez un garçon de 10 ans d'orgine afro-carribéenne nous a conduits à étudier sa famille. Trois sujets symptomatiques ont été identifiés: le garçon de 10 ans et ses 2 oncles maternels âgés de 34 et 41 ans. L'enfant est décèdé à 13 ans dans un tableau de dystonie généralisée et sévère avec complication asphyxique. Une nouvelle mutation faux-sens Trp44Cys du gène TIMM8A a été identifiée chez les 3 sujets atteints. La perte de fonction du gène TIMM8A (Translocase of Inner Mitochondrial Membrane 8A), situé en Xq22, entraine un dysfonctionnement indirect de la chaine respiratoire mitochondriale. En 2007, onze mutations étaient rapportées dans 15 familles. La variabilité phénotypique au sein d'une même famille est rapportée dans la littérature. Les facteurs de cette variabilité pourraient être d'ordre environnemental, ou liés à une hérédité de type oligogénique ou en rapport avec une interaction entre les différentes protéines interagissant au sein de la membrane mitochondriale.

Mots-clés : surdité, TIMM8A.

aP426. NOUVELLE MUTATION P.GLU810X DANS LE GÈNE $A T P 7 B$ RESPONSABLE DE LA MALADIE DE WILSON DANS UNE FAMILLE TUNISIENNE

R. Bhouri (1), M. Gribaa (1), N.S. Abroug (2), A. Mili (1), I. Ben Charfeddine (1), D. H'mida (1), L. Adela (1), O. Mamai (1), T. Belazreg (1), M. Bost (3), A. Saad (1)

(1) Laboratoire de Cytogénétique, de Génétique Moléculaire et de Biologie de la Reproduction Humaines, CHU Farhat Hached, Sousse, Tunisie ; (2) Service de Pédiatrie, CHU Sahloul, Sousse, Tunisie; (3) Centre de Biologie et Pathologie Est - Laboratoire de Neurogénétique Moléculaire et Réseau des Maladies Héréditaires du Métabolisme, Centre de Biologie et Pathologie, Bron, France

Contact : r.bhouri@gmail.com

La maladie de Wilson est une maladie génétique caractérisée par une accumulation toxique de cuivre dans le foie et le système nerveux central. C'une maladie héréditaire rare à transmission autosomique récessive en rapport avec la mutation du gène ATP7B. Le gène ATP7B est localisé sur le chromosome 13 en 13q14.3. Il code pour l'ATPase 7B qui est un transporteur de cuivre. La perte de sa fonction est à l'origine d'une accumulation de cuivre. Environ 320 mutations ont été décrites dont les plus récurrentes sont la $\mathrm{H} 1069 \mathrm{Q}$ et la $\mathrm{R} 778 \mathrm{~L}$ avec une fréquence notable d'hétérozygotes composites. Nous avons étudié dans notre service le cas de trois sæurs âgées de 11, 17 et 26 ans originaire de Sousse en Tunisie. Elles sont issues de parents consanguins et présentent des manifestations cliniques et biologiques évocatrices de la Maladie de Wilson. Le séquençage direct des 21 exons du gène ATP7B chez les trois sœurs a retrouvé une mutation homozygote; $c .2428 \mathrm{G}>\mathrm{T}$ (p.Glu810X), de l'exon 9 du gène ATP7B. Cette mutation n'a jamais était décrite dans la littérature. L'étude de cette mutation sur 200 témoins n'a pas retrouvé de polymorphisme. Le séquençage direct de l'ADN génomique des parents a retrouvé la mutation p.Glu810X à l'état hétérozygote chez les deux parents. Ce diagnostic a été confirmé par la suite grâce à l'analyse d'haplotypes réalisées à l'aide des marqueurs microsatellites D13S296, D13S301, D13S314 et D13S4736. Différentes mutations de l'exon 9 sont fréquemment décrites en Europe méditerranéenne mais nullement rapportées dans la région de l'Afrique du nord ni du Moyen Orient. Une nouvelle mutation s'ajoute donc aux 320 mutations décrites pour le gène ATP7B responsable de la maladie de Wilson et devrait être ciblée par l'analyse moléculaire des patients atteints et de leurs familles notamment dans la région méditerranéenne et nord Africaine.

Mots-clés : Wilson, ATP7B, mutation.

\section{IP427. INSERTION DE NOVO D'UNE SÉQUENCE LINE-1 DANS LE GÊNE ATP7A CHEZ UN PATIENT ATTEINT DE MALADIE DE MENKES}

M.P. Moizard (1, 2), N. Ronce (1, 2), B. Dessay (1), N. Marmin (1), A. Mallet (3), D. Heron (3), N. Horn (4), M. Raynaud (1, 2)

(1) Service de Génétique, CHRU, Tours, France; (2) INSERM U 930 , Tours, France ; (3) Département de Génétique, Groupe Hospitalier PitiéSalpétrière, Paris, France ; (4) Kennedy Center, Glostrup, Danemark Contact : mp.moizard@chu-tours.fr

Les mutations de la séquence codante du gène ATP7A sont à l'origine de la maladie de Menkes, pathologie sévère du métabolisme du cuivre de transmission récessive liée à l'X, dont l'incidence est estimée à 1/250 000 naissances. La pathologie se caractérise cliniquement dès les premiers mois de vie par un retard de croissance et une détérioration neurologique progressive associés à un aspect caractéristique de la peau et des cheveux. Sur le plan biologique, le dosage du cuivre sérique et de la céruloplasmine montre des taux effondrés. Le décès survient dans la majorité des cas avant l'âge de 3 ans. Les mutations à l'origine de la pathologie rapportées à ce jour sont essentiellement des mutations ponctuelles, de tout type, ainsi que des réarrangements impliquant un à plusieurs exons du gène. Quelques anomalies chromosomiques ont également été décrites. Nous décrivons ici le cas d'un patient atteint de maladie de Menkes et porteur de l'insertion de novo d'une séquence d'environ $490 \mathrm{pb}$, survenue dans l'exon 9 du gène. L'élément inséré correspond à la partie 3'd'un rétrotransposon de type LINE-1 (Long-interspersed-element-1) incluant une partie de la queue poly(A) et orienté dans l'antisens par rapport au gène ATP7A. L'insertion génère la duplication d'un site cible de 16 nucléotides, caractéristique de ce type d'évènement. Nous rapportons pour la première fois l'insertion d'un élément LINE-1 dans le gène ATP7A comme mutation causale chez un patient atteint de maladie de Menkes. Ce résultat élargit le spectre des mutations déjà décrites dans cette pathologie.

Mots-clés : ATP7A, Maladie de Menkes, LINE-1.

P428. LES GÈNES CACNA1A ET ATPIA2 SONT FRÉOUEMMENT MUTÉS DANS LA MIGRAINE HÉMIPLÉGIQUE SPORADIQUE À DÉBUT PRÉCOCE

F. Riant (1, 2, 4), A. Ducros (2, 3, 4), C. Ploton (1), C. Barbance (1), C. Depienne $(5,6)$, E. Tournier-Lasserve $(1,2,4)$

(1) Laboratoire de Génétique moléculaire, Hôpital Lariboisière, Paris, France; (2) INSERM U 740, Paris,France: (3) Service de Neurologie, Hôpital Lariboisière, Paris, France : (4) CERVCO, APHP, Hôpital Lariboisière, Paris, France ; (5) Département de Génétique, Cytogénétique et Embryologie, Groupe Hospitalier Pitié-Salpêtrière, Paris, France; (6) INSERM UMR S 679 Neurologie \& Thérapeutique Expérimentale, GH Pitié-Salpêtrière, Paris, France

Contact : florence.riant@lrb.aphp.fr

Introduction : La migraine hémiplégique est un rare type de migraine avec aura qui peut se présenter sous forme familiale (MHF) ou sporadique (MHS). L'analyse des gènes de la MHF dans de précédentes séries de MHS a mis en évidence une faible proportion de patients mutés. Objectif : analyser les gènes de la $\mathrm{MH}$ chez des patients présentant une forme sporadique à début précoce (avant 16 ans). Patients et méthode : 25 patients caucasiens ont été inclus. Chacun d'eux ainsi que leurs 2 parents ont été prélevés. L'âge moyen au moment du diagnostic était de $14,7 \pm 8,2$ et l'âge moyen de la $1^{\text {re }}$ crise de migraine hémiplégique était de $7,5 \pm 3,5$. Le séquençage de CACNA1A et ATP1A2 a été réalisé chez chaque proband et les variants identifiés ont été recherchés chez les parents respectifs. SCN1A a été séquencé chez les patients sans mutation de novo dans CACNA1A ou ATP1A2. Résultats : Vingt trois différents variants ont été identifiés chez 23 patient $(92 \%)$ dont 18 nouveaux. Ces variants sont apparus de novo chez 19 patients $(76 \%)$, ce qui est en faveur de leur caractère délétère. L'analyse du gène SCN1A n'a pas mis en évidence de mutation. Sur les 23 patients mutés, 6 avaient une MHF pure et 17 avaient une FHM associée à des signes neurologiques de type ataxie, épilepsie ou difficultés intellectuelles. Conclusion: l'analyse des gènes FHM est indiquée dans les cas à début précoce, en particulier en présence de signes neurologiques associés. L'identification de 14 nouvelles mutations de novo est d'autre part une aide pour l'interprétation des test moléculaires en pratique diagnostique.

Mots-clés : migraine hémiplégique sporadique, ATP1A2, CACNA1A.

P429. LES MUTATIONS CONVENTIONNELLES SONT ASSOCIÉES À UN PHÉNOTYPE MOINS SÉVÈRE QUE LES EXPANSIONS TRINUCLÉOTIDIQUES DANS LES ATAXIES SPINOCÉRÉBELLEUSES

A. Durr (1, 2), S. Forlani (1), C. Cazeneuve (2), C. Cagnoli (3), K.P. Figueroa (4), D. Lorenzo (5), J. Johnson (6), J. Van de Leemput (6), C. Zaros (2), M. Viemont (2), A. Camuzat (1), S. Benaich (1), A. Singleton (6), L. Ranum (5), S. Pulst (4), A. Brusco (3), E. Leguern (2), A. Brice (1, 2), G. Stevanin $(1,2)$

(1) INSERM/UPMC/NEB, UMR_S975, Génétique des affections neurodégénératives du système nerveux central, Centre de Recherche Institut du Cerveau et de la Moelle, Paris, France: (2) APHP, Département de Généique et Cytogénétique, Groupe Hospitalier Pitié-Salpêtrière, Paris, France ; (3) Department of Genetics, Biology and Biochemistry, University of Torino, Italy : (4) Cedars-Sinai Medical Center, Los Angeles, CA USA ; (5) University of Minnesota, Minneapolis, MN, USA ; (6) National Institute on Aging, NIH, Bethesda, MD, USA

Contact : alexandra.durr@upmc.fr

Les ataxies cérébelleuses autosomiques dominantes forment un groupe hétérogène de pathologies du système nerveux central, aussi bien du point de vue clinique et neuropathologique que génétique. Au moins 30 loci 
(SCAn) ont été localisés. Des répétitions trinucléotidiques CAG ont été incriminées dans 9 gènes et conduisent à des expansions de polyglutamine dans le cas des gènes SCA1, 2, 3, 6, 7 et 17. Plus récemment, des mutations conventionnelles et des réarrangements ont été identifiés dans 8 gènes : SPTBN2/SCA5, TTBK2/SCA11, KCNC3/SCA13, PRKCG/SCA14, ITPR1/SCA15/16, FGF14/SCA27, AFG3L2/SCA28 ainsi que dans le gène codant pour la puratrophine. Nous avons étudié 826 cas index de familles présentant une ataxie cérébelleuse de transmission dominante, collectées entre 1990 et 2008 au sein du groupe hospitalier Pitié-Salpêtrière (Paris) et au sein du réseau clinico-génétique SPATAX (coordonateur: Alexandra Durr). Les gènes retrouvés le plus fréquemment mutés sont ceux associés à des expansions trinucléotidiques CAG codantes : SCA3 $(20,4 \%)$, SCA2 $(9,7 \%), \operatorname{SCA} 1(7,7 \%), \operatorname{SCA} 7(5,7 \%)$ et SCA6 $(1,8 \%)$. Des mutations faux-sens dans les gènes SCA14 $(1,8 \%), \operatorname{SCA} 28(1,6 \%), \operatorname{SCA} 13(1,2 \%)$ et SCA5 $(0,8 \%)$ sont moins fréquentes. Les autres formes sont soit plus rares, comme SCA17 $(0.2 \%)$ et SCA $12(0.2 \%)$, voire absentes de notre cohorte, comme SCA10, SCA27 et le gène codant pour la puratrophine. Dans des séries plus restreintes de patients présentant des formes pures d'ataxie cérébelleuse, des délétions hétérozygotes ont été retrouvées dans 4 cas sur 76 dans le gène SCA15/16 et une mutation non-sens a été retrouvée dans une famille sur 77 dans le gène SCA11. L'étude du phénotype présent chez les patients montre que les expansions CAG ont en commun une progression rapide et sévère de la maladie tandis que les mutations conventionnelles sont associées à un phénotype très lentement progressif, moins sévère avec plus souvent des troubles cognitifs et une atrophie plus marquée du cervelet à l'IRM. Il est donc possible de distinguer cliniquement le type de mutation impliquée au sein de ces pathologies très hétérogènes.

Mots-clés : polyglutamine, ataxie, expansions trinucléotidiques.

\section{P430. IDENTIFICATION D'UN NOUVEAU GENE D'ATAXIE RÉCESSIVE LENTEMENT PROGRESSIVE PAR L'ANALYSE D'UN EFFET FONDATEUR EN ALGÉRIE}

D. H'mida-Ben Brahim (1, 2), A. M'zahem (3), N. Drouot (1), M. Assoum (1), J. Zimmerman (4), L. Ali-Pacha (5), C. Lagier-Tourenne (1), T. Klockgether (4), A. Hamri (3), M. Tazir (5), M. Koenig (1)

(1) Institut de Génétique et de Biologie Moléculaire et Cellulaire, Illkirch, France; (2) Laboratoire de Génétique Moléculaire, de Cytogénétique et de Biologie de la Reproduction Humaines, Hôpital Universitaire Farhat Hached Sousse, Tunisie; (3) Centre Hospitalo-Universitaire Ben Badis, Constantine, Algérie; (4)Klinik und Poliklinik für Neurologie, Universitätsklinikum Bonn, Germany ; (5)Service de Neurologie, Centre HospitaloUniversitaire Mustapha, Alger, Algérie

Contact : dorrahmida@yahoo.fr

Le diagnostic des pathologies héréditaires rares devient de plus en plus difficile à cause de leur origine multigénique. Cette multigénicité est très bien illustrée par les ataxies autosomiques récessives. À ce jour 14 gènes responsables d'ataxies autosomiques récessives ainsi que plusieurs loci ont été identifiés. Cependant plusieurs gènes restent à découvrir. Nous avons dans ce travail adopté une stratégie de clonage positionnel, et plus précisément de cartographie par homozygotie utilisant les puces de génotypage SNP à la recherche de nouveaux gènes impliqués dans les ataxies récessives. Nous avons identifié 12 familles, dont 10 sont d'origine algérienne, homozygotes pour une large région chevauchante sur le chromosome 20 . Seules 5 familles avaient deux ou trois enfants atteints et montraient une liaison significative à ce locus (LOD score $\geq 1,8$, une seule famille avait un LOD score de 3.0 ). L'analyse détaillée des haplotypes avec des marqueurs microsatellites puis avec des puces SNP à très haute densité (Affymterix 6.0), nous a permis d'identifier un petit haplotype fondateur partagé par 4 familles originaires de la région de Sétif dans le nord-est algérien, confirmant ainsi la liaison de ces 4 familles a ce locus du chromosome 20. Le séquençage des gènes de la région critique a permis d'identifier la mutation fondatrice, qui entraîne un décalage du cadre de lecture, et une délétion de l'exon 1 dans la grande famille avec LOD score $=3$, originaire des Émirats Arabes Unis. Les autres familles n'ont pas révélé de mutation dans ce gène, $y$ compris une famille avec un LOD score de 2,6 en faveur de la liaison à ce locus. Deux loci d'ataxie récessive ont déjà été rapportés sur cette région du chromosome 20, SCAR6 (spinocerebellar ataxia recessive 6) et PHARC (Polyneuropathy, Hearing loss, Ataxia, Retinitis pigmentosa and Cataract) qui correspond mieux cliniquement aux 4 familles algériennes et à la famille émiratie. Il est maintenant possible de tester s'il s'agit d'une seule ou de deux entités génétiques.

Mots-clés : ataxie récessive, cartographie par homozygotie, haplotype
G431. LE RÉSEAU SPATAX DANS L'ÉTUDE DES DÉGÉNERESCENCES SPINOCÉRÉBELLEUSES

C. Zaros (2), S. Forlani (1), A. Brice (1, 2), M. Koenig (3), G. Stevanin (1, 2), A. Dürr $(1,2)$ et les membres du réseau Spatax

(1) INSERM/UPMC/NEB, UMR_S975, Génétique des affections neurodégénératives du système nerveux central, Centre de Recherche de l'Institut du Cerveau et de la Moelle Épinière, Paris, France; (2) APHP, Département de Génétique et Cytogénétique, Groupe Hospitalier Pitié-Salpêtrière, Paris, France; (3) IGBMC, Illkirch, France

Contact : cecile.zaros@upmc.fr

Les ataxies cérébelleuses et les paraparésies spastiques (PS) sont deux groupes de pathologies génétiques rares qui résultent d'altérations entrainant des dysfonctionnements de la moelle épinière et/ou du cervelet, et qui sont souvent associées dans leurs formes complexes. Elles sont donc diagnostiquées dans les mêmes centres cliniques et sont étudiées selon des protocoles de recherche similaires. C'est pour cela qu'en 2001, les Drs. Alexandra DÜRR (Paris) et Chantal TALLAKSEN (Oslo), ont créé le réseau SPATAX (Spastic PAraplegia and cerebellar ATAXia) pour rassembler et coordonner les forces et les moyens de recherche au sein du premier réseau international sur ces maladies rares. Le réseau SPATAX compte aujourd'hui 28 équipes de cliniciens et de scientifiques réparties dans 6 pays d'Europe (France, Italie, Portugal, Norvège, Danemark, Grande-Bretagne), 6 pays d'Afrique du Nord et du Proche et Moyen Orient (Algérie, Maroc, Tunisie, Liban, Israël et Arabie Saoudite) et un pays du continent sud-américain (Colombie). Grâce à une collaboration très active, les équipes membres ont pu rassembler le plus grand nombre au monde de familles et cas sporadiques présentant ces pathologies, avec une description très précise des symptômes selon une fiche de diagnostic clinique mise au point au sein du réseau. Ceci a permis d'importants progrès dans la connaissance de ces pathologies très complexes. Depuis 2003, le SPATAX a décrit 4 nouveaux loci génétiques pour les paraparésies spastiques récessives (SPG28, SPG30, SPG32, SPG46), 1 nouveau locus pour les paraparésies dominantes (SPG37), deux dans les ataxies dominantes (SCA25 et SCA31) et 4 pour les ataxies récessives (SAX2, ataxie+épilepsie, MSS et JBTS3). Un travail collaboratif efficace au sein du réseau a permis de découvrir simultanément deux nouveaux gènes pour la forme de paraparésie spastique récessive associée à l'IRM à une réduction caractéristique du corps calleux : les gènes SPG11 et SPG15. De même, dans les ataxies récessives, après l'identification du gène $A O A 2$, responsable comme AOAl, de cas associant une ataxie et une apraxie oculaire, une forme associée à une réduction du coenzyme Q10 a été trouvée mutée dans le gène $A D C K 3$. Ainsi les efforts continus du réseau, afin de rendre accessible le diagnostic génétique au maximum de patients, se sont avérés payants ces dernières années.

Mots-clés : ataxies cérébelleuses, paraparésies spastiques, réseau.

\section{P432. ÉTUDE GÉNÉTIQUE ET MOLÉCULAIRE DE L'ATAXIE PAR DÉFICIENCE EN VITAMINE E ET DE L'ATAXIE DE FRIE- DREICH CHEZ DES PATIENTS MAROCAINS}

H. Dehbi (1), H. Bellayou (1), M.A. Rafai (2), M. Bourezgui (2), I. Slassi (2), S. Nadifi (1)

(1) Laboratoire de Génétique Médicale, Faculté de Médecine et de Pharmacie de Casablanca, Maroc ; (2) Service de neurologie, CHU Ibn Rochd, Casablanca, Maroc

Contact : hind500@yahoo.fr

L'ataxie par déficience en vitamine E (AVED) est une maladie dégénérative autosomique récessive, nettement plus fréquente en Afrique du Nord qu'en Europe. Sur le plan clinique elle est très similaire à l'ataxie de Friedreich (FA) d'où l'autre dénomination «ataxie Friedreich-like», elle pose un vrai problème de diagnostic différentiel. Le diagnostic moléculaire devient particulièrement indispensable, d'abord pour étayer le diagnostic, puis pour détecter au sein de la fratrie les éventuels porteurs sains. Grace à ce test génétique, l'AVED reste la seule ataxie héréditaire curable par supplémentassion en vitamine $\mathrm{E}$. La présente étude a concerné 36 individus, dont 20 propositus et 16 apparentés recrutés dans le cadre de l'enquête familiale. Tous les patients ont été adressés au Laboratoire de Génétique et de Pathologie Moléculaire par le service de neurologie du CHU Ibn Rochd de Casablanca, pour suspicion d'ataxie de Friedreich ou d'AVED. La recherche de la mutation 744delA impliquée dans l'AVED, a été réalisée chez tous les propositus par PCR-RFLP, alors que la recherche de l'expansion GAA retrouvée dans la maladie de Freidriech n'a concerné que les probants négatifs pour la mutation 744delA, et a été faite par Southern Blot, les résultats ont été confirmés par séquençage. Nos résultats ont ainsi confirmé pour 3 familles une AVED, par détection de la mutation 744delA, et une ataxie de Friedreich chez deux autres familles avec un nombre anormal de GAA. Grace à cette étude génétique, 4 patients 
ont été traités par une supplémentation en vitamine E, avec stabilisation et légère amélioration de leur symptomatologie, un conseil génétique avec enquête familiale ont été réalisés. Pour les familles restantes l'étude se poursuit par la recherche des autres mutations, éventuellement d'autres ataxies (télangiectasie, abêtalipoproteinemie...).

Mots-clés : ataxie, mutation 744 delA, répétitions GAA.

\section{P433. UNE HAPLOINSUFFISANCE DU GÈNE DE L'ATAXINE 10 HUMAINE N'EST PAS RESPONSABLE D'ATAXIE SPINO- CÉRÉBELLEUSE DE TYPE 10}

P. Leite (1), A. Jacquette (1), B. Keren (1, 2), C. Depienne (1, 2), A. Durr $(1,2)$, W. Carpentier (3), B. Benyahia (1), G. Ponsot (4), F. Soubrier (1, 3), A. Brice (1, 2), D. Héron (1)

(1) Département de Génétique et Cytogénétique, CGMC, GH Pitié-Salpêtrière, AP-HP, France ; (2) CRICM, Inserm UMR S975, upmc, GH PitiéSalpêtrière, France; (3) Plateforme Post-génomique de la Pitié-Salpêtrière, Inserm, upmc, GH Pitié-Salpêtrière, France; (4) Service de Neuropédiatrie et Pathologie du Développement, Hôpital Armand-Trousseau, APHP, France

Contact : patricia.leite@psl.aphp.fr

Nous présentons une translocation familiale $(2 ; 22)(\mathrm{p} 25.2 ; \mathrm{q} 13.31)$ concernant 3 sœurs nées de parents non apparentés. La $1^{\text {re }}$ et la $3^{\mathrm{c}}$ présentent un retard mental et un syndrome malformatif liés aux monosomie 22qter et trisomie 2 pter. La $2^{e}$ sœur et le père sont porteurs de la translocation à l'état équilibré. Les points de cassure ont été caractérisés précisément par puce $\mathrm{ADN}$ et séquençage : aucun gène n'est touché sur le chromosome 2, mais sur le chromosome 22 , le point de cassure est localisé au niveau du $2^{c}$ intron du gène de l'ataxine 10 , seuls les 2 premiers exons restant sur le dérivé 22 . L'ataxine 10 est le gène de l'ataxie spinocerebelleuse de type 10 (SCA10), maladie neurodégénérative autosomique dominante caractérisée par un syndrome cérébelleux apparaissant entre 20 et 50 ans et dans quelques cas, une épilepsie. L'expansion en tandem à l'état hétérozygote d'un pentanucléotide (ATTCT)n situé dans l'intron 9 est responsable de la maladie quand $n>800$. La fonction exacte de la protéine n'est pour le moment pas connu et le rôle physiopathologique de l'expansion incertain. Des études réalisées chez le rat ont montré qu'un knock down par ARN interférence provoquait une dégénérescence des neurones cérébelleux ce qui serait en faveur d'un mécanisme type perte de fonction chez les patients SCA10 (J Biol Chem 279: 35542-50) À l'inverse des études chez des souris null hétérozygotes n'ont montré aucun phénotype clinique et anatomo-pathologique ce qui serait en défaveur d'un mécanisme type perte de fonction chez les patients SCA10 (Neurology $67 ; 607-13$ ). Dans notre famille, le père et la $2^{\mathrm{e}}$ fille ont une déficience de l'ataxine 10 à l'état hétérozygote mais ont un examen neurologique normal. Une apparition plus tardive de l'ataxie ne peut être exclue chez la fille âgée de 23 ans, mais est par contre très improbable chez le père âgé de 57 ans étant donné la survenue antérieure à 50 ans dans les cas décrits. Cette observation est donc en défaveur d'une haploinsuffisance de l'ataxine 10 humaine provoquée par l'expansion du pentanucléotide (ATTCT)n chez les patients SCA10.

Mots-clés : ataxie spino cerebelleuse, ATXN10, puces SNP.

D434. GENOME SCANS IN AUTOSOMAL DOMINANT CEREBELLAR ATAXIAS IDENTIFY NEW LOCI

G. Stevanin (1, 2), E. Di Gregorio (3), S. Forlani (1), J. Koht (5), M. Turcotte Gauthier (4), E. Mundwiller (1), C. Tesson (1), B. Van De Warrenburg (6), A. Filla (7), B. Borroni (8), W. Carpentier (9), D. Zelenika (10), A. Durr (1, 2), C. Tallaksen (5), P. Cossette (4), A. Brice (1, 2), A. Brusco (3) and the EUROSCA consortium

(1) INSERM/UPMC/NEB, UMR_S975, Génétique des affections neurodégénératives du système nerveux central, Centre de Recherche de l'Institut du Cerveau et de la Moelle Épinière, Paris, France; (2) APHP, Département de Génétique et Cytogénétique, Groupe Hospitalier Pitié-Salpêtrière, Paris, France; (3) Department of Genetics, Biology and Biochemistry, University of Turin, Italy; (4) Research center of the CHUM, Hôpital Notre-Dame, Centre of Excellence in Neuromics of the Montréal University, Québec, Canada ; (5) Oslo University Hospital, Ullevaal, Oslo, Norway: (6) University Nijmegen Medical Centre, the Netherlands ; (7) Federico II University, Naples, Italy ; (8) Department of Neurology, University of Brescia, Italy; (9) Plateforme P3S, Groupe Hospitalier PitiéSalpêtrière, Paris, France; (10) Centre National de Génotypage, Évry, France

Contact : giovanni.stevanin@upmc.fr

In order to identify new loci/genes in autosomal dominant cerebellar ataxias (ADCA), members of the EUROSCA consortium collected DNA and clinical information of 31 families with at least 5 affected patients. Only two families presented with a theoretical LOD score of +3 independently. Genome scans were performed at the Centre National de Genotypage or in our local platform (P3S) using 6K Illumina SNP arrays. Linkage was evaluated with the MERLIN software using an incomplete penetrance set at $80 \%$. As expected, only two families generated a significant multipoint LOD score $>+3$ at two new candidate regions: 1) a Norwegian family showing early-onset cerebellar ataxia with spasticity $(\mathrm{Zmax}=3.7)$ and 2$)$ an Italian family with pure ADCA (SCA31, Zmax $=3.4$ ). Three additional EUROSCA families were also found putatively linked to the SCA31 locus. In addition, this locus was mapped independently in one Canadian ADCA kindred with erythrokeratodermia $(\mathrm{Zmax}=5.3)$. Twelve other families presented with maximal multipoint LOD scores ranging from 1.5 to 2.8 to single or multiple regions on the genome. Systematic analysis of microsatellites markers in the positive regions in these 12 most informative families, allowed, in most cases, to either exclude several of these regions or to reduce their size. The initial hypothesis of a major new ADCA locus has been ruled out by the fact that most of the identified candidate intervals do not overlap but SCA31 might account for up to 4 out of 31 of our collected families.

Mots-clés : ataxia, SCA31, cerebellum.

P435. SCA3 HÉTÉROZYGOTE COMPOSITE, PHÉNOTYPE CLINIQUE ET HYPOTHESES PHYSIOPATHOLOGIQUES

P. Charles (1, 2, 3), C. Cazeneuve (4), C. Jardel (5), F. Salachas (3), C. Fauchard (6), A. Dürr $(1,3)$

(1) APHP, Département de Génétique et Cytogénétique, UF de Génétique Clinique, Groupe Hospitalier Pitié-Salpêtrière, Paris, France; (2) INSERM/UPMC/NEB, UMR_S975, Génétique des affections neurodégénératives du système nerveux central, Centre de Recherche Institut du Cerveau et de la Moelle, Groupe Hospitalier Pitié-Salpêtrière, Paris, France ; (3) Fédération des maladies du système nerveux, Groupe Hospitalier PitiéSalpêtrière, Paris, France ; (4) APHP, Département de Génétique et Cytogénétique, UF de Neurogénétique Moléculaire et Cellulaire, Groupe Hospitalier Pitié-Salpêtrière, Paris, France; (5) APHP, Service de Biochimie Métabolique, Biochimie Endocrinienne et Oncologique, Groupe Hospitalier Pitié-Salpêtrière, Paris, France; (6) Consultation de Neurologie, Hôpital de Mantes, Mantes La Jolie, France

Contact : charles.perrine@psl.aphp.fr

Une patiente âgée de 48 ans a été adressée pour des troubles de l'équilibre d'aggravation progressive depuis l'âge de 37 ans. Les antécédents familiaux étaient compatibles avec une transmission récessive sans censure dans une fratrie issue d'une union consanguine. L'examen neurologique montrait un syndrome cérébelleux statique et cinétique, une ophtalmoplégie complexe avec un nystagmus dans les regards latéraux, une amyotrophie distale, des ROT vifs sans signe de Babinski, une hypopallesthésie distale, des fasciculations de la langue. Le score d'ataxie SARA (Scale for the Assessment and Rating of Ataxia) était à 25/40 en 2008. L'IRM cérébrale montrait une atrophie à prédominance vermienne, l'EMG une polyneuropathie axonale sensitive avec des signes neurogènes moteurs proximaux. Le bilan étiologique d'ataxie récessive s'est révélé négatif (AF, $\mathrm{AOA} 1, \mathrm{AOA} 2, \mathrm{AVED})$ de même que le bilan métabolique. Compte tenu du phénotype clinique, une analyse moléculaire des principaux gènes responsables des ataxies cérébelleuses autosomiques dominantes (SCA1, 2, $3,6,7$ et 17) a été réalisée, et a montré une expansion pathologique de triplets CAG (respectivement 63 et $65 \mathrm{CAG}$ ) sur les deux allèles au locus SCA3/MJD, une situation exceptionnellement rapportée dans la littérature. Une biopsie musculaire réalisée dans le bilan métabolique a montré un déficit en complexe III de la chaîne respiratoire. Le séquençage du gène du cytochrome B seul gène mitochondrial codant pour une sous unité du complexe III était négatif. En conclusion, les sujets homozygotes pour une expansion de CAG dans le gène SCA3 ont un phénotype clinique proche de celui des hétérozygotes avec un âge de début similaire et un phénotype clinique proche de celui décrit pour les grandes expansions CAG avec notamment une atteinte du motoneurone. Le mécanisme du déficit en complexe III de la chaîne respiratoire mitochondriale (primitif ou secondaire) reste à étudier et ouvre des perspectives dans la physiopathologie de ces affections neurodégénératives.

Mots-clés : SCA3, hétérozygote composite, phénotype clinique.

تP436. SCA15 LIÉE AUX DÉLÉTIONS DU GÈNE ITPRI: FRÉQUENCE ET PHÉNOTYPE DANS UNE COHORTE DES FAMILLES AVEC ATAXIE DOMINANTE

C. Marelli $(1,2,3)$, J. Johnson (4), J. Van De Leemput (5), C. ThauvinRobinet (6), F. Tison (7), F. Picard (8), B. Huttin (9), C. Marescaux (10), C. Tranchant (10), J. Boulliat (11), N. Vandemberghe (12), S. Brique (13), 
H. Dollfus (14), E. Ollagnon (15), J. Hardy (4), G. Stevanin (1, 2, 3, 16), A. Singleton (5), A. Dürr (1, 2, 3, 16), A. Brice (1, 2, 3, 16)

(1) Université Pierre-et-Marie-Curie,Paris6, Centre de Recherche de l'Institut du Cerveau et de la Moelle Épinière, UMR-S975, Paris, France; (2) Inserm, U975, Paris, France ; (3) AP-HP, Hôpital de la Salpetrière, Service de Génétique et Cytogénétique, Paris, France; (4) Department of Molecular Neuroscience, Institute of Neurology, UCL, London, UK ; (5) Molecular Genetics Unit, National Institute of Aging, NIH, Bethesda, USA ;(6) Service de Génétique, CHU Dijon, France; (7) Service de Neurologie, Hôpital du Haut Lévêque, Université Bordeaux 2, Pessac, France ; (8) Service de Neurologie, UHMS, Geneva, Suisse; (9) Service de Neurologie, Centre Hospitalier Jean-Monnet, Épinal, France ; (10) Service de Neurologie, CHRU Strasbourg, France; (11) Service de Neurologie, Hôpital de Fleyrat, Bourg-en-Bresse, France ; (12) Service de Neurologie, CHU Lyon, France ; (13) Clinique Convert, Bourg-en-Bresse, France: (14) Laboratoire de Physiopathologie des Syndromes Rares Héréditaires, AVENIR-Inserm, EA3949, Université Louis Pasteur, Strasbourg, France ; (15) Service de Génétique, Hôpital de la Croix Rousse, Lyon, France; (16) CNRS, UMR 7225, Paris, France Contact : alexandra.durr@upmc.fr

Background: SCA15 est une forme d'ataxie cérébelleuse autosomique dominante (ADCA) récemment identifiée et liée a des anomalies dans le gène ITPR I, qui code pour le récepteur type 1 de l'inositol-triphosphate. Dans la plupart des cas il s'agit de délétion de taille variable, une seule famille SCA15 avec une mutation faux sens a été décrite. Objective : déterminer la fréquence et le phénotype des patients SCA 15. Patients: 333 familles avec ADCA, exclues pour les ataxies avec expansions de trinucléotides CAG (SCA1, 2, 3, 6, 7, 17), et pour les mutations conventionnelles responsable de SCA5 (232 cas index), SCA11 (61 cas index), SCA14 (197 cas index), SCA13 (202 cas index), SCA28 ( 238 cas index), and puratrophine ( 232 cas index) ont été analysées. Méthodes : recherche des délétions dans le gène ITPRI par PCR quantitative (258 cas index) or par puces SNP à haute densité ( 75 cas index). Résultats : une délétion partielle d'ITPRI à l'état hétérozygote a été retrouvée dans $5 / 333$ familles $(1,5 \%)$, correspondant à 11 patients. L'âge de début allait de 18 à 66 ans (en moyenne $33 \pm 15$ ans) ; la durée de maladie était comprise entre 3 et 43 ans (en moyenne $22 \pm 14$ ans). Le symptôme de début était principalement une ataxie de la marche ; seulement un patient débutait avec un tremblement intentionnel des membres supérieurs. Le phénotype des patients SCA15 était une ataxie cérébelleuse axiale et des membres très lentement progressive avec dysarthrie cérébelleuse. Les mouvements oculaires étaient caractérisés par une poursuite saccadique et un nystagmus, surtout horizontal et dans les regards latéraux. Un seul patient avait un syndrome pyramidal réflexe des membres inférieurs. Deux patients présentaient des troubles de la déglutition. La progression était lente car seulement deux patients, avec une durée de la maladie $>40$ ans, nécessitaient une aide pour la marche. L'IRM cérébrale montrait une atrophie cérébelleuse globale ou à prédominance vermienne. Conclusions : les délétions d'ITPR 1 sont rares et représentent $<1 \%$ de l'ensemble des ADCA mais environ $4,5 \%$ de l'ensemble des formes pures. Le phénotype SCA15 est une ataxie cérébelleuse pure, à évolution très lentement progressive mais avec un âge de début très variable.

Mots-clés : Ataxie Cerebelleuse Dominante (ADCA), SCA15, ITPR1

\section{IP437. FREQUENCY, PHENOTYPE AND MUTATIONAL SPEC-} TRUM OF ARSACS

I. Coupry (1), E. Maurat (1), C. Blanchard (1), S. Forlani (2), C. Zaros (2), M. Anheim (2), A. Durr (2), A. Brice (2), B. Arveiler (1), G. Stevanin (2), C. Goizet (1)

(1) Laboratoire de Génétique Humaine, Université Bordeaux 2, Bordeaux, France ; (2) INSERM/UPMC UMR_S975, Hôpital Pitié-Salpêtrière, Paris, France

Contact : isabelle.coupry@u-bordeaux2.fr

Autosomal recessive spastic ataxia of Charlevoix-Saguenay (ARSACS : MIM 270550) is a neurodegenerative disorder characterized by early-onset cerebellar ataxia with spasticity and peripheral neuropathy. This disorder was first described in the isolated Charlevoix-Saguenay region of Quebec, but nowadays, it is known to occur worldwide. This study presents the identification of ARSACS in patients recruited from the SPATAX network. Material and Methods: To determine the frequency of ARSACS among the patients, we included in this study patients with spastic ataxia, early-onset before the age of 25 and for whom Friedreich's ataxia was previously excluded through DNA mutation analysis. Molecular analysis were performed on 92 patients, by PCR amplification and direct sequencing of all the 9 coding exons and the intron-exon boundaries of the SACS revealed 4 patients with homozygous mutations (one missense, two non sense and one frameshift mutation), and 7 with compound heterozygous mutations. The remaining 14 patients were presenting only one mutation. Discussion: Among the 92 selected patients, only 11 could be found to carry two SACS mutations $(12 \%)$. Even if the missense mutations concerned evolutionnary highly conserved aminoacids and were not found in 200 control chromosomes, their real pathogenicity remain uncertain until confirmation with DNA from affected siblings or relatives. Concerning the 14 patients with a unique SACS mutation, we cannot exclude that the second one remained undetected because it resides in parts of the gene that have not been analyzed (regulatory regions upstream of the gene, introns), or because it results from a partial deletion of the gene. Finally, the absence of mutations might be due to genetic heterogeneity for the spastic ataxia phenotype, and the involvement of other loci like SPAX2 or SPAX3/ARSAL

Mots-clés : spastic ataxia, SACS

\section{P438. ARBRES DÉCISIONNELS POUR LES ANALYSES DE GÉNÉTIQUE MOLÉCULAIRE DANS LE CADRE DES ATAXIES CÉRÉBELLEUSES AUTOSOMIQUES DOMINANTES}

Réseau des Laboratoires de Diagnostic Moléculaire des Maladies Génétiques Neurologiques, Musculaires, Neurosensorielles et Retards Mentaux, M. Viémont (1), A. Dürr $(2,3,4)$, E. Leguern $(1,2)$, C. Cazeneuve (1)

(I) Unité Fonctionnelle de Neurogénétique Moléculaire et Cellulaire, Département de Génétique et Cytogénétique, Groupe Hospitalier PitiéSalpêtrière, Assistance Publique, Hôpitaux de Paris, Paris, France ; (2) Inserm UMR_S975, Groupe Hospitalier Pitié-Salpêtrière, Paris, France ; (3) Centre de Référence des Maladies Neurogénétiques, Groupe Hospitalier Pitie-Salpêtrière, Assistance Publique, Hôpitaux de Paris, Paris, France: (4) Unité Fonctionnelle de Génétique Clinique, Département de Génétique et Cytogénétique, Groupe Hospitalier Pitié-Salpêtrière, Assistance Publique, Hôpitaux de Paris, Paris, France

Contact : cecile.cazeneuve@psl.aphp.fr

L'élaboration d'arbres décisionnels pour les analyses de génétique moléculaire et pour les prérequis cliniques amenant à la prescription de ces analyses constitue une démarche indispensable pour les laboratoires de génétique moléculaire afin d'améliorer la qualité du service rendu tout en tenant compte de la limitation des moyens humains et des moyens de fonctionnement dont ils disposent. Un des objectifs du réseau des laboratoires de diagnostic moléculaire des maladies génétiques neurologiques, musculaires, neurosensorielles et retards mentaux est de rédiger ces arbres décisionnels, action favorisée pour les aspects analytiques par le rapprochement des acteurs des différents laboratoires de génétique moléculaire et, pour l'établissement des prérequis cliniques, par une collaboration avec les centres de référence maladies rares. Les ataxies spinocérébelleuses autosomiques dominantes, cliniquement et génétiquement hétérogènes, sont caractérisées par une ataxie progressive de la marche, de la posture et des extrémités, une dysarthrie et/ou des troubles oculomoteurs, en relation avec une dégénérescence cérébelleuse visible à l'IRM. La prévalence est d'environ 1/50000. Les mutations des gènes les plus fréquemment impliqués (SCA1, SCA2, SCA3, SCA6, SCA7, SCA17, DRPLA, impliqués dans environ $50 \%$ des cas) consistent en des expansions de triplets CAG. Il existe une corrélation inverse entre le nombre de répétitions et l'âge de début de la maladie, qui se situe le plus souvent entre 30 et 50 ans. L'instabilité des répétitions est à l'origine d'un phénomène d'anticipation qui explique, à l'extrême, les rares formes juvéniles voire infantiles dues à la présence de grandes ou très grandes expansions. Il existe par ailleurs d'autres formes d'ataxies cérébelleuses : les ataxies autosomiques récessives, liées à d'autres gènes (notamment FRDA, AOA1, AOA2); les formes non génétiques d'ataxies, notamment d'origine toxique ou métabolique, s'accompagnant ou non d'une atrophie cérébelleuse. Compte tenu des différentes étiologies (génétiques ou non) et, pour les formes familiales, de l'hétérogénéité génétique, l'établissement de prérequis cliniques pour la prescription des analyses de génétique moléculaire est particulièrement nécessaire. Compte tenu des données cliniques et des résultats des études moléculaires princeps, des critères de décisions simples, basés sur le mode de transmission, sur la présence d'une atrophie cérébelleuse à l'IRM et sur l'âge de début, ont permis d'élaborer des prérequis cliniques pour la demande d'analyses génétiques. De même, la pathologie moléculaire des gènes impliqués a conduit à l'élaboration de stratégies diagnostiques. La confrontation de ces arbres décisionnels avec l'expérience d'un laboratoire ayant testé plus de 1000 patients appartenant à plus de 850 familles différentes a permis leur validation a posteriori, montrant notamment que la transmission dominante du phénotype est un critère essentiel.

Mots-clés : arbre décisionnel, ataxie dominante. 
GP439. GENETIC HETERONENEITY IN KARAK SYNDROME DEMONSTRATED BY LINKAGE ANALYSIS AND MUTATION SCREENING IN PANK2 AND PLA2G6 GENES IN 8 CASANGUINEOUS SAUDI ARABIAN FAMILIES

H. Azzedine (1), Mundwiller (1), A. Khan (2), E.A. Aldriss (3), S.A. Elmalik (3), M.M. Kabiraj (4), M.A.M. Salih (5), G. Stevanin (1) (1) INSERM/UPMC UMRS 975, Hôpital de la Pitié-Salpêtrière, Paris, France ; (2) King Khaled Eye Specialist Hospital, Riyadh, Saudi Arabia ; (3) Department of Physiology, College of Medicine, King Saud University, Riyadh, Saudi Arabia ; (4) Division of Clinical Neurophysiology, Department of Neuroscience, military Hospital, Riyadh, Saudi Arabia ; (5) Division of Pediatric Neurology, College of Medicine, King Saud University, Riyadh, Saudi Arabia

Contact : azzedine.hamid@yahoo.fr

A hetererogeneous group of severe neurological disorders like Aceruloplasminaemia, Neuroferritinopathy, Hallervorden-Spatz syndrome, HARP syndrome, and Friedreich ataxia involves excess brain iron accumulation. The "eye of the tiger" sign is a common neuroradiological finding in neurodegeneration with iron brain accumulation type 1 and 2 (NBIA1, 2), infantile neuroaxonal dystrophy (INAD) as well as in Karak syndrome (KS). Mutations in PANK2 and PLA2G6 genes were implicated in NBIA, INAD and KS disorders. We describe 8 consanguineous Saudi families with Karak syndrome. These consisted of 13 affected individuals ( 6 males and 7 females, aged 19 Months-24 years). Onset ranged between about 8 months and 7 years with difficulty in walking or progressive cerebellar ataxia and spasticity associated, later, with extrapyramidal signs, intellectual decline and axonal form of Charcot-Marie- Tooth disease (CMT2). Ambulation was lost between $41 / 2$ and 15 years. One male patient died at 24 years. Ophthalmic evaluations revealed abnormal vertical saccades and pursuit. Brain MRI showed iron deposition in the putamen in all patients aged more than 4 years. Five of these families were genotyped for PANK2 and PLA2G6 loci using 10 microsatellite markers. PANK2 locus was excluded in all the families while assignment of the families to the PLA2G6 locus was established by homozygocity mapping in 4 of them. Using direct sequencing of the PLA2G6 gene we identified, at a homozygous state, 3 novel mutations and one other already reported in the 4 linked families. One of these 5 families was neither linked to PLA2G6 nor to PANK2 genes demonstrating further genetic heterogeneity. Three new other consanguineous families with the same phenotype and belonging to the same country are under investigation. The final results will be presented during the meeting.

Mots-clés : KARAK Syndrome, PANK2, PLA2G6.

घP440. MUTATIONS DE TARDBP DANS LES DÉMENCES FRONTO-TEMPORALES ASSOCIÉES À UNE SCLÉROSE LATÉRALE AMYOTROPHIQUE (DFT-SLA)

L. Guillot-Noël (1), L. Benajiba (1), A. Camuzat (1), I. Le Ber (1, 2, 3), M. Lacoste (4), C. Thomas-Anterion (5), V. Golfier (8), P. Couratier (4), S. Legallic (6), S. Millecamps (1), L. Lacomblez $(3,5,7)$, F. Salachas (3), D. Campion (6), V. Meininger (3), A. Brice (1, 2, 3, 7, 9, 10), and the French clinical and genetic research network on FTD/FTD-MND

(1) CRICM INSERM UMR S 975, Hôpital de la Salpêtrière, Paris, France ; (2) Centre de référence des démences rares, Hôpital de la Salpêtrière, Paris, France ; (3) Fédération des Maladies du système nerveux, Hôpital de la Salpêtrière, Paris, France ; (4) Service de neurologie, CHU, Limoges, France ; (5) Service de Neurologie, CHU Bellevue, Saint-Étienne, France ; (6) INSERM U614 \& Département de Neurologie, CHU Charles-Nicolle, Rouen, France; (7) Université Pierre-et-Marie-Curie, Paris, France ; (8) Service de Neurologie, CHU, Rennes, France; (9) INSERM U610, Hôpital de la Salpêtrière, Paris, France; (10) Département de Génétique, Cytogénétique et Embryologie, Hôpital de la Salpêtrière, Paris, France Contact : lena.guillot-noel@upmc.fr

Les démences fronto-temporales (DFT) sont des pathologies neurodégénératives rares conduisant à des troubles du comportement et du langage. Elles peuvent être associées à une sclérose latérale amyotrophique (DFTSLA). Plusieurs études ont montré l'implication du gène TARDBP, codant pour la protéine TDP-43, comme responsable de la maladie chez certains patients atteints de SLA sans démence. TDP-43 est une ribonucléoprotéine de $43 \mathrm{k}$ Da impliquée dans l'épissage des ARN et la régulation de la transcription. Cette protéine est le composant majeur des inclusions neuronales observées dans les SLA, DFT et les DFT-SLA. À ce jour on compte une vingtaine de mutation de l'exon 6 du gène TARDBP correspondant au domaine hnRNP de TDP-43 dans des formes pures de SLA. Nous avons séquencé cet exon sur 199 patients atteints de DFT-SLA dont 102 avec une forme familiale de la pathologie et 97 avec une forme sporadique.
Nous avons également analysé 569 contrôles pour valider nos résultats. Une recherche de réarrangements a été réalisée chez 58 patients par QMPSF. Nous avons identifié trois mutations faux-sens de l'exon 6 du gène TARDBP par séquençage. Une mutation faux-sens hétérozygote Gly295Ser a été identifiée chez deux patients, l'un atteint d'une DFT-SLA familiale et l'autre d'une DFT-SLA sporadique. L'analyse des haplotypes au locus TARDBP a mis en évidence un effet fondateur dans ces deux familles. Une mutation faux-sens homozygote I383V a été identifiée chez un patient atteint d'une forme familiale de DFT-SLA. Cette dernière avait déjà été mise en évidence mais à l'état hétérozygote chez un patient atteint d'une SLA familiale. Le tableau clinique (phénotype : âge de début) ne se distingue pas du tableau habituellement observé chez des patients porteurs de mutations hétérozygotes. Par ailleurs aucun réarrangement n'a été détecté par QMPSF, ce qui suggère que s'ils existent ces évènements sont rares. Il s'agit de la première étude mettant en évidence l'implication de ce gène dans certaines formes de DFT. La fréquence des mutations est comparable à celle des SLA pures soit $1,3 \%$. Par cette étude nous montrons l'existence de nouvelles mutations impliquant le gène TARDBP dans les DFT-SLA.

Mots-clés : démences fronto-temporales, DFT-SLA, TARDBP.

IP441. ANALYSE DU GÈNE PGRN ET DOSAGE PLASMATIQUE DE LA PROGRANULINE DANS LES DÉGÉNERESCENCES LOBAIRES FRONTOTEMPORALES

A. Camuzat (1), F. Lamari (2), F. Clot (3, 7), C. Jardel (2), D. Hannequin (4), D. Campion (4), F. Pasquier (5), V. Deramecourt (5), C. Duyckaerts (1), A. Laquerrière (6), B. Dubois (7), I. Le Ber (1, 7), A. Brice (1)

(1) CRicm INSERM UMRS975, Paris, France; (2) Service de biochimie métabolique, Hôpital Pitié-Salpêtrière, Paris, France; (3) Département de génétique, cytogénétique et embryologie, Hôpital Pitié-Salpêtrière, Paris, France ; (4) INSERM U614, Rouen, France; (5) Service de neurologie, CHU, Lille, France : (6) Service de neuropathologie, CHU, Rouen, France: (7) Centre de Références des démences rares, Hôpital PitiéSalpêtrière, Paris, France

Contact : agnes.camuzat@upmc.fr

Les dégénérescences lobaires frontotemporales (DLFT) sont des maladies neurodégénératives rares de l'adulte se manifestant par des troubles comportementaux et du langage. 30 à $50 \%$ des DLFT sont autosomiques dominantes. À ce jour, 4 gènes sont impliqués dans cette pathologie (MAPT, PGRN, VCP et CHMP2B), MAPT et PGRN expliquant environ 20 à $30 \%$ des formes monogéniques. Le gène PGRN est situé sur le bras long du chromosome 17 (17q21-22) et est composé de 13 exons. Il code pour la progranuline qui est le précurseur des granulines. C'est un facteur de croissance qui joue un rôle dans la réparation tissulaire, la réponse inflammatoire et la tumérogenèse. Nous avons analysé ce gène par séquençage et QMPSF chez 622 patients atteints de DLFT (404 sporadiques, 218 familiaux). Nous avons identifié 25 mutations chez 34 patients. Ce sont des mutations nonsens, des mutations situées dans les sites d'épissage ou bien des mutations qui entrainent un décalage du cadre de lecture. Elles aboutissent toutes à la synthèse d'ARNs messagers possèdant un codon stop prématuré qui sont alors dégradés par le mécanisme de dégradation des ARN non-sens. Les mutations du gène PGRN entraînent donc une haploinsuffisance et la progranuline est ainsi exprimée à $50 \%$ seulement de son taux normal. De récentes études ont montré que cette diminution d'expression entraînait une diminution d'environ $1 / 3$ du taux de progranuline dans le sang chez les individus porteurs d'une mutation du gène PGRN. Nous avons réalisé un dosage plasmatique par la technique ELISA chez 9 individus porteurs de mutations PGRN, 13 témoins ( 2 individus DLFT porteurs d'une mutation dans le gène MAPT, 4 patients atteints de maladie d'Alzheimer, 3 individus DLFT dont la cause est inconnue et 4 contrôles). Seuls les patients porteurs d'une mutation PGRN montrent une diminution importante du taux plasmatique avec une valeur autour de $50 \mathrm{ng} / \mathrm{ml}$ alors que le taux normal varie entre 146 et $300 \mathrm{ng} / \mathrm{ml}$ (seuil à $100 \mathrm{ng} / \mathrm{ml}$ ). Le dosage de la progranuline plasmatique est donc une bonne méthode pour détecter des patients DLFT porteurs d'une mutation PGRN et représente un moyen rapide et peu onéreux pour orienter vers l'analyse de ce gène.

Mots-clés : DLFT, PGRN, mutations.

-9442. DÉMENCE TARDIVE DE TYPE ALZHEIMER DUE À UNE NOUVELLE VARIATION DANS LA RÉGION LINKER DE LA PROTÉINE TWINKLE

A. Echaniz-Laguna (1), J.B. Chanson (1), J.M. Wilhelm (2), F. Sellal (3), M. Chassagne (4), M. Mayençon (4), M. Mohr (5), C. Tranchant (1), B. Mousson de Camaret (4)

(1) Département de Neurologie, Hôpital Civil, Strasbourg, France ; (2) Service de Médecine Interne, Centre Hospitalier Régional, Altkirch, 
France : (3) Service de Neurologie, Centre Hospitalier Régional, Colmar, France; (4) Service des Maladies Héréditaires du Métabolisme, Centre de Biologie et de Pathologie Est, CHU Lyon, Bron, France ; (5) Département d'Anatomo-Pathologie, Hôpital de Hautepierre, Strasbourg, France

Contact : benedicte.mousson-de-camaret@chu-lyon.fr

Les variations dans le gène PEO1 codant pour l'hélicase mitochondriale Twinkle sont habituellement la cause d'une ophtalmoplégie progressive externe de transmission autosomique dominante (adPEO) associée à des délétions multiples de l'ADN mitochondrial (ADNmt). Nous décrivons cinq patients issus de deux familles alsaciennes non apparentées qui ont présenté une affection complexe d'évolution progressive, de transmission autosomique dominante, associant PEO, surdité, myopathie, dysphonie, dysphagie, neuropathie sensorielle puis démence tardive de type $\mathrm{Alz}$ heimer. L'analyse moléculaire des biopsies de muscle par PCR longue a révélé des délétions multiples de l'ADNmt. Une nouvelle variation (p.Arg374Trp) du gène PEO1 a été mise en évidence chez ces patients, dans la région linker de la protéine Twinkle, indispensable à la réplication de l'ADNmt. Cette variation affecte le codon 374 , strictement conservé à travers les espèces, et induit un changement sévère dans la structure de la région linker, d'une importance capitale pour l'activité hélicase de la protéine Twinkle. Ces observations démontrent pour la première fois qu'une variation de Twinkle est suffisante pour détériorer les fonctions cérébrales et soulignent le rôle central des altérations de l'ADNmt dans la physiopathologie des syndromes démentiels. Echaniz-Laguna A. et al. Neurogenetics 2009, in press

Mots-clés : démence tardive, Twinkle, ADN mitochondrial.

IP443. ANALYSE DU GÈNE FUS DANS LES DÉMENCES FRONTOTEMPORALES ASSOCIÉES OU NON À UNE SCLÉROSE LATÉRALE AMYOTROPHIQUE

I. Le Ber (1, 2, 3), O. Broustal (1), A. Camuzat (1), L. Guillot-Noël (1), S. Millecamps (1), N. Guy (4), F. Salachas (2), D. Hannequin (5), L. Lacomblez (2), D. Campion (5), V. Meininger (3), B. Dubois (2, 3, 6), A. Brice $(1,2,7)$

(1) CR-ICM INSERM UMRS_975, Hôpital de la Pitié-Salpêtrière, Paris, France: (2) Fédération des maladies du système nerveux, Hôpital de la Pitié-Salpêtrière, Paris, France: (3) Centre de Référence des Démences Rares, Hôpital de la Pitié-Salpêtrière, Paris, France; (4) Service de neurologie, CHU Clermont-Ferrand, France ; (5) INSERM U614 \& Département de Neurologie, CHU Charles Nicolle, Rouen, France ; (6) INSERM U610, Hôpital de la Salpêtrière, Paris, France ; (7) Département de Génétique, Cytogénétique et Embryologie, Hôpital de la Salpêtrière, Paris, France

Contact : isabelle.leber@upmc.fr

Les démences frontotemporales (DFT) sont des maladies dégénératives de l'adulte se manifestant par des troubles comportementaux. Elles peuvent être associées à une sclérose latérale amyotrophique (SLA) entrainant une faiblesse des membres, des troubles respiratoires et de la déglutition (DFTSLA). En 2009, des mutations du gène FUS ont été identifiées chez des patients atteints de SLA sans démence. FUS est une RNA-binding protein impliquée dans la régulation de la transcription, l'épissage et le transport des ARNm. La majorité des mutations sont localisées dans l'exon 15 qui code pour le domaine $\mathrm{C}$-terminal riche en glycines de la protéine. Récemment, il a été montré que certaines formes de DFT $(10 \%)$ sont caractérisées par la présence d'inclusions neuronales FUS-positives, suggérant que ce gène pourrait être aussi impliqué directement dans les DFT. Nous avons séquencé les 15 exons du gène FUS chez 318 patients atteints de DFT pure $(n=145)$, ou de DFT-SLA $(n=173)$. Des mutations des gènes MAPT, GRN, CHMP2B, VCP (responsables de DFT pure) et de SOD1, ANG, TARDBP (responsables de SLA pure) avaient été préalablement exclues par séquençage. Nous avons identifié deux variants exoniques présents à l'état hétérozygote chez deux patients. Une mutation faux-sens (c. $1562 \mathrm{G}>\mathrm{A}, \mathrm{p} . \mathrm{R} 521 \mathrm{H}$ ) a été identifiée dans l'exon $15 \mathrm{chez}$ un patient atteint de DFT-SLA autosomique dominante. Cette mutation a été identifiée dans d'autres études chez des patients atteints de SLA pure. Le proposant a débuté la maladie par des troubles comportementaux (désinhibition, jovialité, indifférence émotionnelle, stéréotypies) à l'âge de 71 ans. Des symptômes moteurs de SLA sont apparus quelques mois plus tard. Un nouveau variant faux-sens (c.1369G>C, p.G457R) a été identifié dans l'exon 13 du gène FUS chez un patient présentant une forme pure de DFT (sans SLA) et ayant des antécédents familiaux de DFT. Ce variant, qui n'est pas présent chez les témoins, touche une glycine conservée au cours de l'évolution, localisée dans le domaine G-rich de FUS. Aucune mutation n'a été identifiée chez des patients sans histoire familiale, montrant que la pénétrance est plus élevée que pour les mutations TARDBP. La fréquence des mutations est peu élevée dans cette série $(0.3 \%)$, de même que dans le sous-groupe des patients avec DFT-SLA $(0,6 \%)$. Elle est comparable à une autre étude portant sur des patients d'origine française atteints de SLA $(0.8 \%)$. Des progrès récents ont été faits dans le domaine de la génétique des DFT et des SLA avec l'identification récente de nouveaux gènes dans ces deux pathologies. L'identification de mutations du gène FUS dans les DFT montre qu'il existe un overlap entre les causes génétiques des DFT et des SLA, et suggère un rôle probable de la protéine FUS au niveau des neurones du cortex frontotemporal. Les mécanismes par lesquels elle est impliquée dans la dégénérescence neuronale restent cependant à établir.

Mots-clés: démence frontotemporale, FUS, sclérose latérale amyotrophique.

口P444. FORMES GÉNÉTIQUES DES MALADIES À PRIONS : DEUX MUTATIONS DU RESIDU 211 DE LA PROTÉINE PRION CONDUISENT À DEUX MALADIES DISTINCTES

K. Peoc'h (1), E. Levavasseur (2), E. Delmont (3), A. De Simone (4), I. Laffont-Proust (2), N. Privat (2), Y. Chebaro (5), J.P. Brandel $(6,7)$, E. Morain (6), J.J. Hauw (8), S. Chasseigneaux (1), P. Derreumaux (5), J.L. Laplanche $\left(1^{*}\right), S$. Haîk $(2,7,8)$

(1) AP-HP, Service de Biochimie et Biologie Moléculaire associé au CNR "ATNC », Hôpital Lariboisière Paris, France et EA 3621 Université Paris Descartes, Paris, France; (2) Centre de Recherche de I'Institut du Cerveau et de la Moelle épiniere, UMR-S975 et Université Pierre-et-MarieCurie, Paris, France; (3) Centre référent en Pathologies Neuromusculaires et SLA, Hôpital Archet 1, Nice, France; (4) Department of Chemistry University of Cambridge, Cambridge, United Kingdom; (5) Laboratoire de Biochimie Théorique, UPR 9080 CNRS et Université ParisDiderot Paris, France ; (6) CNRS, UMR 7225, Paris, France ; (7) Cellule Nationale de référence des Maladies de Creutzfeldt Jakob, France; (8) AP-HP, Laboratoire de Neuropathologie R. Escourolle, G.H. Pitié-Salpêtrière, Paris, France

Contact : jean-louis.laplanche@lrb.aphp.fr

Les maladies à prion humaines constituent un groupe de maladies neurodégénératives hétérogènes, caractérisées par l'accumulation dans le système nerveux d'une forme anormalement conformée de la protéine prion (PrP) endogène associée à une perte neuronale et une astrocytose. La plus fréquente de ces maladies rares est la maladie de Creutzfeldt-Jakob (MCJ) qui existe sous une forme sporadique, génétique ou acquise et se caractérise par une démence précoce d'évolution rapide. Le syndrome de GertsmannStraussler-Scheinker (GSS) et l'insomnie familiale fatale sont également des formes génétiques très rares. Ces formes génétiques, de transmission autosomique dominante, concernent $15 \%$ des patients. Les différents phénotypes typiques sont associés à différentes mutations du gène PRNP (MIM\#176640), qui code la protéine prion. Plus d'une cinquantaine de mutations ponctuelles ou d'insertions ont été répertoriées à ce jour. Nous décrivons un exemple original de variabilité phénotypique de ces maladies sous la forme de deux mutations distinctes affectant le résidu 211 , situé dans le domaine C-terminale de PrP, et responsables de deux maladies phénotypiquement différentes sur les plans clinique, neuropathologique et biochimique. Nous présentons les cas d'un patient porteur de la mutation p.Glu211 Asp associée à un GSS, ainsi que de deux patients non apparentés présentant la mutation p.Glu211Gln et atteint de MCJ. Par modélisation moléculaire, nous avons mis en évidence le fait que ces deux substitutions d'un résidu très conservé pouvaient modifier différemment la stabilité des protéines mutées. L'étude biochimique des protéines accumulées dans le système nerveux central montrent des propriétés physico-chimiques bien distinctes pouvant être reliées aux différences cliniques, biochimiques et neuropathologiques observées. Ces résultats sont en accord avec l'hypothèse protéique qui propose qu'un changement conformationel de la protéine prion soit l'élément clé du développement de ces maladies.

Mots-clés : prion, Creutzfeldt Jakob, syndrome de Gertsmann Straussler Scheinker.

- 445 . LOCALISATION DU $3^{\circ}$ LOCUS IMPLIQUE DANS LE TREMBLEMENT MYOCLONIQUE CORTICAL FAMILIAI AVEC ÉPILEPSIE SUR LE CHROMOSOME 5P

D. Bouteiller (1), E. Magnin (3), G. Stevanin (1), C. Saint-Martin (1), M. Vidailhet $(4,2)$, E. Apartis (5), E. LeGuern $(1,2)$, P. Labauge (6), L. Rumbach (3), C. Depienne (1, 2)

(1) Inserm, UMR_S975, Université Pierre-et-Marie-Curie-Paris6, CNRS 7225. Centre de Recherche, Institut du Cerveau et de la Moelle, Paris, France ; (2) AP-HP, Assistance Publique Hôpitaux de Paris, Groupe Hospitalier Pitié-Salpêtrière, Département de Génétique et Cytogénétique, U.F. de Neurogénétique Moléculaire et Cellulaire, Paris, France; (3) Département de Neurologie, CHU Jean Minjoz, Besançon, France ; (4) 
Fédération de Neurologie, Groupe Hospitalier Pitié-Salpêtrière, Paris, France; (5) Département de Physiologie, Hôpital Saint-Antoine, Paris, France ; (6) Département de Neurologie, CHU Nîmes, France

\section{Contact : delphine.bouteiller@upmc.fr}

Le tremblement myoclonique cortical familial avec épilepsie (TMCFE) est un syndrome autosomique dominant rare caractérisé par des myoclonies corticales associées chez $40 \%$ des patients à une épilepsie. Le tremblement cortical est le premier signe à apparaitre, généralement à l'âge adulte. Deux loci, sur les chromosomes 8q23.3-q24.11 et 2p11.1-q12.2, ont été décrits pour le TMCFE mais aucun gène n'a été identifié à ce jour. Dans cette étude, nous rapportons une grande famille d'origine française avec TMCFE, pour laquelle les deux loci connus été exclus. Les patients présentent : 1) des crises généralisées tonico-cloniques précédant les myoclonies corticales ( $31 \%$ des cas) ; 2 ) des crises partielles $(31 \%)$ et 3 ) des migraines avec auras visuelles $(37 \%)$. Un tour du génome incluant 14 membres de la famille a été effectué à l'aide de puces SNP 6K (Illumina). Le calcul LOD-score multipoint a montré une seule région positive en 5p15.31-p15.1 avec un LOD-score maximal de 3,66. Le génotypage des 45 membres de la famille disponibles à l'aide de 13 marqueurs microsatellite a permis de définir une région de $9,31 \mathrm{Mb}$ entre les marqueurs D5S580 et D5S2096, les LOD scores bi-points atteignant 6,3 et 6,2 à thêta égal 0 pour D5S486 et D5S1380. Cet intervalle contient 23 gènes, dont la plupart sont exprimés dans le cerveau mais aucun ne code pour un canal ionique. Deux gènes candidats, SEMA5A et CTNND2, ont été analysés par séquençage direct mais aucune mutation n'a été identifiée. L'identification de ce troisième locus ouvre la perspective d'identifier le premier gène en cause dans cette pathologie hétérogène génétiquement.

Mots-clés : tremblements corticaux, épilepsie, chromosome 5p.

EP446. ÉTUDE D'UNE COHORTE DE PATIENTS RENNAIS PRÉSENTANT DES ANOMALIES DE MIGRATION NEURONALE : DIAGNOSTIC CLINIQUE ET STRATÉGIES DE GÉNÉTIQUE MOLÉCULAIRE

C. Quélin (1), L. Pasquier (1), P. Loget (2), S. Jaillard (3), C. Dubourg (4), S. Mercier (1), C. De la Rochebrochard (1), C. Tréguier (5), C. FalletBianco (6), C. Beldjord (7), J. Chelly (7), N. Bahi-Buisson (8), S. Odent (1)

(1) Service de génétique clinique, Rennes, France ; (2) Service d'anatomie et cytologie pathologiques, Rennes, France; (3) Service de cytogénétique et biologie cellulaire, Rennes, France; (4) Service de biochimie et génétique moléculaire, Rennes, France ; (5) Service de radiologie et imagerie médicale, Rennes, France ; (6) Service d'anatomie pathologique, Hôpital Saint-Anne, Paris, France; (7) Service de biochimie et génétique moléculaire, Hôpital Cochin, Paris, France; (8) Service de neurologie pédiatrique, Hôpital Necker Enfants Malades, Paris, France

Contact : chloe1512@yahoo.fr

Les anomalies de migration neuronale appartiennent au spectre des dysgénésies corticales, pathologies développementales liées à un trouble de la mise en place du cortex et des circonvolutions cérébrales, dont les causes sont très diverses (génétiques et environnementales). Elles sont responsables d'un retard psychomoteur et d'une encéphalopathie de sévérité variable, associées à une épilepsie souvent pharmaco-résistante, et peuvent être ou non accompagnées d'anomalies des autres structures cérébrales. Elles regroupent principalement les lissencéphalies ou spectre agyrie/ pachygyrie/hétérotopies laminaires sous-corticales, les hétérotopies neuronales et les polymicrogyries. Certaines des ces anomalies sont de mieux en mieux caractérisées sur le plan moléculaire. En effet, des mutations dans différents gènes codant pour des protéines tubulines (TUBA1A et TUBB2B) ont très récemment été impliquées chez des patients présentant des dysgénésies corticales diverses (pachygyrie ou polymicrogyrie) mais associant des caractéristiques communes, renforçant l'hypothèse d'un rôle des microtubules dans la migration neuronale. Aux vues de ces récentes découvertes, il nous a paru intéressant de faire le point sur ces pathologies par l'étude de 36 dossiers du CHU de Rennes et des données de la littérature, afin de revoir les aspects cliniques, radiographiques ou anatomopathologiques, nosologiques et génétiques des dysgénésies corticales. Parmi ces sujets, on distingue 12 fœtus (issus d'interruptions médicales de grossesse) et 24 patients nés vivants. L'ensemble de ces sujets (ou leurs parents) ont été vus au moins une fois à la consultation de génétique du $\mathrm{CHU}$. On retient de cette étude une grande hétérogénéité clinique et génétique. En effet, parmi les sujets, $50 \%$ ont une pachygyrie, $33 \%$ polymicrogyrie et $19 \%$ ont des hétérotopies neuronales. Ces anomalies peuvent être associées chez un même patient et la majorité $(64 \%)$ a d'autres malformations neurologiques. Sur le plan génétique, des anomalies ont été mises en évidence dans $25 \%$ des cas, et impliquent 4 gènes : LIS1, DCX, TUBA1A et TUBB2B. Cette étude a de plus permis de poursuivre les études moléculaires chez certains sujets mais il apparaît que les causes de ces malformations corticales sont encore souvent inconnues et que des progrès sont à faire dans leur compréhension. Par ailleurs, nous proposons, en accord avec les données de la littérature, une stratégie de diagnostic génétique devant un trouble de migration neuronale, différente selon le type de malformations. Enfin, cette étude nous permet de préciser le conseil génétique et de discuter de l'importance de l'imagerie anténatale, en particulier de l'IRM cérébrale fœtale, dans le dépistage des anomalies de gyration et l'évaluation de leur gravité.

Mots-clés : retard mental, dysgénésie corticale, migration neuronale.

\section{- 4 447. PATHOLOGIE MOLÉCULAIRE DE LA MALADIE DE} LAFORA (EPM2) EN FRANCE

G. Lesca (1), N. Boutry-Kryza (1), B. De Toffol (2), M. Milh (3), D. Steschenko (4), M. Lemesle-Martin (5), L. Maillard (6), G. Foletti (7), G. Rudolf (8), J. Mancini (3), C. Thauvin (9), E. Hirsch (8), A. Calender (1), P. Genton (10)

(1) Service de génétique moléculaire et médicale, hôpital Édouard-Herriot et Université Claude Bernard Lyon 1, Lyon, France ; (2) Service de neurologie, CHU Bretonneau, Tours, France; (3) Service de neurologie pédiatrique, hôpital Timone-enfants, Marseille, France; (4) Médecine infantile, hôpital Davigny, Lavigny, Suisse; (8) Service de neurologie, Hopitaux universitaires de Strasbourg, Strasbourg, France ; (9) Centre de génétique, hôpital d'enfants, CHU de Dijon, France ; (10) Centre SaintPaul, Marseille, France

Contact : gaetan.lesca@chu-lyon.fr

Introduction - La maladie de Lafora (ML) ou EPM2 est une maladie neurodégénérative à transmission autosomique récessive débutant dans l'enfance ou l'adolescence. Elle est caractérisée par un syndrome myoclonique d'aggravation progressive, des crises généralisées ou partielles, en particulier occipitales, un déclin cognitif et une évolution fatale en 5 à 10 ans. La ML est causée par les mutations d'au moins deux gènes: EPM2A et NHLRCl, situés sur des bras différents du chromosome 6. Nous rapportons la première série de patients étudiés sur le plan génétique en France. Matériel et méthodes - L'ADN de 36 patients provenant de différents centres français a été étudié entre 2005 et 2009. Les gènes EPM2A et NHLRC1 ont été étudiés par séquençage des régions exoniques et recherche de grands réarrangements par qPCR. Pour la mutation d'épissage, c.101-2G $>\mathrm{A}$, L'étude de l'ADNc du gène EPM2A a été effectuée par RT-PCR et séquençage. Plusieurs microsatellites intra- ou juxta-géniques ont été étudiés chez les patients présentant des mutations communes. Résultats - Le diagnostic moléculaire a été confirmé chez $10(27 \%)$ des cas index, dont 7 EPM2A et 3 EPM2B. Aucune consanguinité n'était rapportée dans ces familles. Tous les patients sauf 2 sont hétérozygotes composites. Concernant le gène EPM2A, 5/6 patients d'origine française sont porteurs d'au moins 1 exemplaire de la mutation p.Arg $241 \mathrm{X}$ qui est présente sur $50 \%$ des allèles. Tous les patients porteurs de cette mutation sauf 1 partagent le même haplotype. Quatre mutations de ce gène n'ont jamais été rapportées, dont la mutation, c.101-2G>A, qui conduit à un épissage anormal de l'exon 2. Concernant le gène NHLRC1, 2 patients sont porteurs de la mutation p.Pro69Ala qui est associée à des haplotypes différents. Une autre mutation n'a jamais été rapportée. Discussion -Nous retrouvons, chez les patients français, la grande hétérogénéité allélique classique de la ML, toutefois quelques mutations sont largement prédominantes. Notre étude montre que contrairement à ce qui était admis dans la littérature, la forme EPM2A est plus fréquente que la forme EPM2B en France. Ceci semble principalement lié à la prévalence de la mutation p.Arg24IX, qui est également très fréquente en Espagne. La seule patiente de la série qui était originaire de l'étranger (Afrique du Nord) étaient porteuses de deux mutations privées du gène EPM2A. Tous les patients chez lesquels nous avons retrouvé des mutations présentaient une forme classique de ML. Nous n'avons pas retrouvé de différence évidente en termes de sévérité entre les patients EPM2A et EPM2B. Une patiente atteinte d'EPM2B a présenté une évolution particulièrement rapide avec décès au bout de 2 ans.

Mots-clés : lafora, epilepsie myoclonique progressive, EPM2.

IP448. THE FIRST GENOME-WIDE SCAN IN A TUNISIAN FAMILY WITH GENERALIZED EPILEPSY WITH FEBRILE SEIZURE PLUS (GEFS+)

N. Fendri- Kriaa (1), N. Louhichi (1), F. Kammoun (2), G. Chabchoub (1), A. Rebai (3), C. Triki (2), F. Fakhfakh (1)

(1) Laboratoire de Génétique Moléculaire Humaine, Faculté de Médecine de Sfax, Tunisia : (2) Unité de Neuropédiatrie de Sfax, Faculté de Médecine de Sfax, Tunisia; (3) Unité de Bioinformatique et de Biostatistique, Centre de Biotechnologie de Sfax, Tunisia

Contact: fnourhene@yahoo.fr 
Generalized Epilepsy with Febrile Seizure plus (GEFS+) is an autosomal dominant disorder characterized by the association of febrile and afebrile seizures. Five responsible genes of GEFS+ were identified: SCNIB, SCN1A, SCN2A, GABRG2 and GABRD respectively on 19q13.1, $2 q 21-33,2 q 24,5 q 34$ and $1 \mathrm{p} 36.3$ chromosomes. Two novel susceptibility loci for GEFS+ at 2p24 and 8p23-p21 were also reported indicating the genetic heterogeneity of this disorder. The aim of this report is to identify the loci responsible for GEFS + in a large Tunisian family by performing a $10 \mathrm{cM}$ density genome-wide scan. The highest multipoint LOD score (1.04) was found for D5S407 in the absence of recombination. Two other interesting regions were found around marker D19S210 (LOD $=0.799$ ) and D7S484 (LOD = 0.61) markers. For fine mapping, additional markers in two regions on $5 \mathrm{q} 13.3$ and $7 \mathrm{p} 14.2$ were analyzed and positive LOD scores for both loci were obtained. Sequencing of SCN1B, which is the candidate gene for FS and epilepsy localized in the chromosome 19q13.1, showed the absence of any causal mutation. Our findings emphasized the genetic heterogeneity of febrile seizures. Furthermore, epilepsy in association with febrile seizures might occur in this Tunisian family owing to an interaction between at least two genes.

Mots-clés : GEFS+, genome scan, febrile seizures.

\section{घP449. ÉPIDÉMIOLOGIE MOLÉCULAIRE DU GÈNE HTT: L'EXPÉRIENCE DU LABORATOIRE DE NEUROGÉNÉTIQUE DU GROUPE HOSPITALIER PITIÉ-SALPÊTRIËRE DANS LE CADRE DU DIAGNOSTIC MOLÉCULAIRE DE LA MALADIE DE HUNTINGTON}

S. Noël (1), O. Russaouen (1), M. Viémont (1), E. Leguern (1, 2), A. Dürr $(2,3)$, C. Cazeneuve (1)

(1) Unité Fonctionnelle de Neurogénétique Moléculaire et Cellulaire, Département de Génétique et Cytogénétique, Groupe Hospitalier PitiéSalpêtrière, Assistance Publique - Hôpitaux de Paris, Paris, France ; (2) Inserm UMR_S975, Groupe Hospitalier Pitié-Salpêtrière, Paris, France ; (3) Unité Fonctionnelle de Génétique Clinique, Département de Génétique et Cytogénétique, Groupe Hospitalier Pitié-Salpêtrière, Assistance Publique - Hôpitaux de Paris, Paris, France

Contact : cecile.cazeneuve@psl.aphp.fr

La maladie de Huntington est une affection neurodégénérative touchant environ $1 / 10000$ personnes en France. Elle est liée dans $99 \%$ des cas à une amplification pathologique de triplets CAG dans l'exon 1 du gène HTT. En fonction du nombre de CAG (n), on distingue : les allèles non associés à la pathologie, sans risque $(\mathrm{n} \leq 26)$ ou à risque $(27 \leq \mathrm{n} \leq 35)$ d'instabilité méiotique; les allèles associés à la pathologie, avec pénétrance incomplète $(36 \leq n \leq 39)$ ou complète $(n \geq 40)$. Il existe une corrélation entre le nombre de triplets et l'âge d'apparition des premiers signes cliniques. Par ailleurs, les amplifications de grande taille, rares, sont associées à des formes juvéniles, sévères et d'évolution rapide de la maladie. Nous rapportons ici la distribution de 1667 allèles du gène HTT selon l'expérience de notre laboratoire qui réalise le diagnostic moléculaire de la maladie de Huntington depuis fin 2004 pour des indications de confirmation de diagnostic (DC), diagnostic présymptomatique (DPS) et diagnostic prénatal (DPN). Nous avons étudié 719 familles, soit 896 individus, dont 509 adressés pour un DC, 342 pour un DPS et 45 conjoints. Ces personnes représentent un total de 1667 allèles indépendants. 48 DPN ont également été réalisés. $\mathrm{n} \leq 26$. L'allèle portant 17 CAG est le plus fréquent ( $33 \%)$ des 1209 allèles normaux. Nous avons identifié 20 allèles portant 17 triplets dont l'avant dernier est un CAA ( $6 \%$ des allèles portant 17 triplets). $27 \leq \mathrm{n} \leq 35$. Cinquante-sept allèles à risque d'instabilité méiotique ont été identifiés. Pour 38 individus, le second allèle est normal (n ?26) alors que 19 portent un second allèle pathologique $(n \geq 36)$. Trois transmissions d'allèles potentiellement instables ( 27 ou 29 CAG) ont été recensées à l'occasion de DPN. Dans les trois cas, les allèles ont été transmis sans instabilité par le père. $36 \leq \mathrm{n} \leq 39$. Un allèle à $38 \mathrm{CAG}$ a été identifié chez un patient et 3 allèles à 39 répétitions ont été identifiés dont 2 à l'occasion d'un DPS. $n \geq 40$. L'allèle le plus fréquent $(n=43)$ représente $17 \%$ des 397 allèles recensés. $94,5 \%$ des allèles portent de 40 à 50 répétitions, 17 allèles portent de 51 à 60 triplets $(4,3 \%)$. Cinq allèles portent plus de $60 \mathrm{CAG}$ : dans quatre cas $(\mathrm{n}=62,62,66,70)$, les patients présentent un âge de début dans l'adolescence; pour un patient ayant débuté la maladie à l'âge de 4 ans, le nombre de triplet est supérieur à 120. Quarante-six transmissions d'allèles pathologiques (parent-enfant étudiés dans notre laboratoire) ont été dénombrées. Les observations sont conformes à ce qui est rapporté dans la littérature : l'instabilité est d'autant plus fréquente et plus ample que le nombre de triplets chez le parent porteur est élevé et que le parent porteur est un homme. Un seul cas de très grande instabilité a été observé : un père portant 49 triplets a transmis un allèle portant plus de $120 \mathrm{CAG}$. Au total, ce travail présente l'épidémiologie moléculaire du gène HTT sur un échantillon représentatif de la population française.

Mots-clés : Huntington, HTT, épidémiologie.

P450. ÉVALUATION DE LA MESURE DE L'ACTIVITÉ GEF DU FACTEUR EIF2B COMME OUTIL D'AIDE AU DIAGNOSTIC DES EIF2B-PATHIES

A. Huyghe (1, 2), L. Horzinski (1, 2), R. Schiffmann (3), M.C. Cardoso $(1,2,4)$, P. Blanc $(1,2,4), C$. Gonthier (1), O. Boespflug-Tanguy $(1,2$, 5), A. Fogli $(1,2,4)$

(1) INSERM U931 CNRS 6247 Génétique Reproduction et Développement (GReD), Faculté de Médecine, Clermont-Ferrand, France ; (2) Université ClermontI, UFR Médecine, Clermont-Ferrand, France: (3) Institute of Metabolic Disease, Baylor Research Institute, Dallas, Texas, United States; (4) Service de Biochimie Médicale et Biologie Moléculaire, $\mathrm{CHU}$ de Clermont-Ferrand, Clermont-Ferrand, France; (5) Service de Génétique Médicale, Centre de Référence Leucodystrophies, CHU de ClermontFerrand, Hôpital Hôtel-Dieu, Clermont-Ferrand, France.

Contact : anne.fogli@inserm.u-clermont1.fr

Des mutations dans les cinq gènes EIF2B1-5 codant les sous-unités du facteur eucaryote d'initiation de la traduction $2 \mathrm{~B}$ (eIF2B) ont été mises en cause dans un groupe hétérogène de leucodystrophies, les eIF2B-pathies. Le phénotype de ces pathologies est très variable : allant de formes sévères congénitales à des formes modérées avec un début de la maladie à l'âge adulte et une détérioration neurologique lente ou absente. La sévérité est corrélée à l'âge de début de la maladie et trois formes ont été décrites: la forme sévère infantile précoce (âge de début $<2$ ans), la forme classique infantile tardive (âge de début entre 2 et 5 ans), et la forme juvénile/adulte modérée (âge de début $>5$ ans). La plus fréquente est la forme classique initialement décrite, appelée syndrome CACH/VWM (Childhood ataxia with central hypomyélination/vanishing white matter disorder). L'imagerie par résonance magnétique (IRM) révèle un profil spécifique servant au diagnostic avec une atteinte diffuse et symétrique de la substance blanche (SB) cérébrale, cavitaire. À ce jour, la sélection des patients pour le séquençage long et coûteux des gènes EIF2B1-5 se fait sur des critères clinicoIRM. Le facteur eIF2B active le facteur eIF2 grâce à son activité d'échange de guanine (GEF). Nous avons montré une diminution de cette activité GEF dans les lymphoblastes de patients porteurs de mutations EIF2B1-5 en comparaison de patients contrôles, cette diminution étant corrélée à l'âge de début de la maladie. Afin d'évaluer la valeur diagnostique et pronostique de la mesure de cette activité GEF dans ces pathologies, nous avons effectué le test dans les cellules de 63 patients porteurs de mutations EIF2B1-5 en comparaison de 18 patients contrôles sains, de 19 patients avec un tableau clinico-IRM compatible avec le syndrome CACH/VWM mais non porteurs de mutations eIF2B (CACH/VWM-like), et de 19 patients porteurs d'autres leucodystrophies identifiées. Nos résultats montrent une diminution de l'activité GEF dans les cellules des patients mutés avec une spécificité de $100 \%$ lorsque la valeur seuil est fixée $<77,5 \%$. Pour les patients avec leucodystrophie et avec une activité GEF $>77,5 \%$, des mutations dans les gènes EIF2B1-5 sont retrouvées dans $15 \%$ des cas, ce chiffre s'élève à $27 \%$ si les patients ont un tableau clinico-IRM CACH/VWM-like. Ainsi, le séquençage des gènes EIF2B1-5 est indiqué pour les patients cliniquement $\mathrm{CACH} / \mathrm{VWM}$ avec une activité GEF $>77,5 \%$, principalement pour les formes modérées. La corrélation trouvée initialement entre la sévérité et la diminution de l'activité GEF est restreinte au groupe de patients sévèrement atteints avec un âge de début $<1,5$ ans, et dont l'activité GEF mesurée est toujours $<55 \%$ (marqueur pronostique). Pour les autres formes de sévérité, d'autres facteurs doivent probablement moduler l'effet des mutations sur le phénotype. Nos résultats permettent de valider ce test rapide comme un outil d'aide au diagnostic et au pronostic des eIF2B-pathies.

Mots-clés : leucodystrophies, diagnostic, pronostic.

IP451. SYNDROME D'AICARDI-GOUTIÈRES : EXPÉRIENCE DU LABORATOIRE DE RÉFÉRENCE POUR LE DIAGNOSTIC MOLÉCULAIRE DES LEUCODYSTROPHIES (CHU DE CLERMONT-FERRAND)

C. Nachury (1), P. Blanc (1, 2), A. Dion (1), I. Creveaux $(1,2)$

(1) Laboratoire de Biochimie et Biologie Moléculaire, Faculté de Médecine, CHU de Clermont-Ferrand, France; (2) CNRS, UMR 6247; Clermont Université ; INSERM, U931, Faculté de Médecine, GReD, ClermontFerrand, France

Contact : pblanc@chu-clermontferrand.fr

Le syndrome d'Aicardi-Goutières est une maladie rare à transmission autosomique récessive (exceptionnellement dominante). Les formes 
d'apparition prénatale ou néonatale peuvent mimer les infections congénitales de type TORCH. Elles se manifestent par une encéphalopathie subaiguë associée à une épilepsie (53\%), des engelures des extrémités (43\%) et des épisodes aseptiques fébriles $(40 \%)$ et évoluent en quelques mois vers une déficience intellectuelle, une microcéphalie et un syndrome pyramidal. Les formes plus tardives, débutant après 1 an, sont de sévérité moindre : absence de microcéphalie, fonctions cognitives relativement préservées, survie au-delà de 10 ans. Le diagnostic repose sur l'aspect de leucodystrophie kystique associée à des calcifications des noyaux gris centraux et de la substance blanche à l'IRM, ainsi que sur la lymphocytose et l'élévation transitoire de l'INF alpha dans le LCR. Cinq gènes ont été identifiés comme porteurs de mutations responsables de ce syndrome: TREX1, RNASEH2A, RNASEH2C (formes plutôt précoces), RNASEH2B (formes plutôt tardives) et plus récemment SAMHD1. Depuis 2007, 34 patients ont été analysés au Laboratoire de Référence de diagnostic moléculaire des leucodystrophies et 12 identifiés comme porteurs de mutations dans l'un des 4 gènes étudiés (TREX1, RNASEH2A, B, C). La majorité de ces mutations (16/22 soit $73 \%$, versus $47 \%$ selon Rice et al., 2007) impliquent le gène RNASEH2B avec une forte récurrence de la mutation p.Ala177Thr dans l'exon 7 (15/22 soit $68 \%$, versus $29 \%)$ pour laquelle 6 patients sont homozygotes. Trois patients sont porteurs de mutations dans TREX1 (soit $25 \%$ des patients versus $30 \%$ ). À ce jour, nous n'avons identifié aucune mutation dans les gènes RNASEH2A et C. Toutefois, l'implication de RNASEH2A est rare (4\% des mutations décrites) et les mutations de RNASEH2C ont été décrites surtout chez des familles pakistanaises. Un des problèmes diagnostiques est l'identification d'une seule mutation à l'état hétérozygote, en l'occurence la mutation récurrente p.Ala177Thr, héritée d'un parent lui-même indemne, ce qui suggère un défaut de détection de la seconde mutation en trans. Dans les 2 cas observés, aucune autre mutation n'a été retrouvée dans les régions analysées du gène RNASEH2B et nos résultats préliminaires indiquent que les transcrits correspondant à l'allèle non porteur de p.Ala177Thr sont de taille et de séquence normales, donc que la seconde mutation, si elle existe, n'affecte pas l'épissage. Nous n'observons pas de corrélations génotype-phénotype notables. En particulier, sur 7 enfants présentant une forme précoce, 6 sont mutés dans RNASEH2B, tandis que sur 5 enfants atteints d'une forme tardive, 3 sont mutés dans RNASEH2B. Il semble donc prioritaire de rechercher la mutation récurrente p.Ala177Thr, puis de séquencer le gène RNASEH2B, quelle que soit la forme clinique. Tous les patients mutés ont une présentation clinique conforme à la description de Rice et al., 2007.

Mots-clés : syndrome d'Aicardi-Goutières, gènes TREX1 et complexe RNASEH2, leucodystrophie.

\section{P452. LES MUTATIONS DE SPG11 SONT RARES DANS LES} LEUCODYSTROPHIES DE CAUSE INDÉTERMINÉE

I. Coupry (1), E. Pierre (2, 3), C. Vaurs-Barriere (2), E. Kaphan (4), B. Arveiler (1, 7), O. Boespflug-Tanguy (2, 6), G. Stevanin (5), C. Goizet $(1,7)$

(1) Laboratoire de Génétique Humaine, Université Victor-Segalen Bordeaux 2, Bordeaux, France; (2) INSERM UMR931 CNRS 6647, Clermont University, GReD, Clermont-Ferrand, France ; (3) Laboratoire de Cytogénétique Médicale, Clermont-Ferrand, France

(4) Service de Neurologie, Hôpital de la Timone, Marseille, France ; (5) INSERM/UPMC UMR_S975, Groupe Hospitalier Pitié-Salpêtrière, Paris, France; (6) Service de Neuropédiatrie, Hopital Robert Debré, Paris, France; (7) Service de Génétique Médicale, CHU Bordeaux, Bordeaux, France

Contact : cyril.goizet@chu-bordeaux.fr

Les paraplégies spastiques héréditaires sont des affections très hétérogènes caractérisées par une spasticité progressive, une faiblesse musculaire et des troubles de la sensibilité profonde des membres inférieurs. Les formes autosomiques récessives (PSAR) sont encore mal connues et seulement 6 gènes parmi les 14 loci identifiés. Une entité clinique fréquente parmi les PSAR associe une atteinte cognitive, une atrophie du corps calleux (ACC) et des anomalies de la substance blanche (PSAR-ACC). SPG11 est la forme la plus fréquente de PSAR-ACC. Nous avons recherché des mutations du gène KIAA1840/SPG11, codant pour la spatacsine, par séquençage direct des 40 exons codants et des jonctions intron-exon dans une série de 35 malades atteints d'anomalies de la substance blanche ayant fait porter le diagnostic de leucodystrophie de cause indéterminée. Nous avons identifié trois mutations délétères de KIAA1840/SPG11 chez 2/35 malades. Il s'agit de mutations tronquantes : deux correspondent à des petites insertions/délétions déjà décrites dans la littérature aboutissant à un décalage du cadre de lecture, et la troisième à une nouvelle mutation d'épissage. Dans les deux cas mutés, une ACC et une paraplégie spastique d'aggravation progressive venaient compléter le tableau clinique. Les mutations de KIAA1840/SPG11 peuvent donc rarement expliquer des phénotypes étiquetés leucodystrophies. La fréquence de SPG11 est d'environ $6 \%$ dans notre série de 35 malades. Les anomalies de la substance blanche évocatrice d'une leucodystrophie sont cependant associées dans les deux cas à une ACC qui doit orienter vers l'analyse du gène KIAA1840/SPG11.

Mots-clés : SPG11, leucodystrophie, spasticité.

IP453. CRIBLAGE DES GÈNES PARK DANS LES FORMES PRÉCOCES DE LA MALADIE DE PARKINSON

A. Honoré $(1,2), C$. Dupuits $(1,2)$, C. Condroyer $(1,2)$, E. Lohmann $(1$, 2), C. Cazeneuve (3), A. Troiano (1, 2), S. Lesage (1, 2), A. Dürr $(1,2,3)$, A. Brice $(1,2,3)$ et le réseau PDG

(1) INSERM, UMR_S975 (exUMR_S679), Paris, France ; (2) Université Pierre-et-Marie-Curie-Paris6, UMR_S975 CRicm, Pitié-Salpêtrière, Paris, France: (3) AP-HP, Hôpital Pitié-Salpêtrière, Département de Génétique et Cytogénétique, Paris, France

Contact : alexis.brice@upmc.fr

Introduction : La maladie de Parkinson (MP) est une maladie neurodégénérative, la $2^{e}$ la plus fréquente, après la maladie d'Alzheimer, avec une prévalence d'environ $2 \%$ chez les personnes âgées de plus de 65 ans. La MP se manifeste cliniquement par la triade : akinésie, rigidité et tremblement de repos avec une bonne réponse à la L-Dopa. Ce désordre est dû à la perte de neurones dopaminergiques de la substance noire. Depuis une dizaine d'années, au moins 13 loci et 9 gènes sont associés aux rares formes monogéniques de la MP, dont 5 gènes validés par de nombreuses études génétiques : 3 gènes sont plutôt associés aux formes autosomiques récessives [Parkine (PARK2), PINK1 (PARK6) et DJ-1 (Park7)] et 2 autres, aux formes autosomiques dominantes [SNCA (PARK1/4) et LRRK2 (PARK8)]. But du travail : Estimer la fréquence relative des mutations dans les gènes de la Parkine, PINK 1 , DJ-1, SNCA et LRRK2 dans une série de formes précoces de la MP (âge de début de la maladie $\leq 45$ ans), d'origine européenne, plus particulièrement française et nord-africaine. Patients et Méthodes :

Patients : 203 cas familiaux et 345 cas isolés avec au moins un atteint ayant un âge de début de la maladie $\leq 45$ ans, d'origine européenne, plus particulièrement française et nord-africaine.

Méthodes:- Séquençage des 12 exons et région promotrice de la Parkine, 8 exons de PINK1, 7 exons de DJ-1 et l'exon 41 de LRRK2 contenant la mutation fréquente G2019S, sur séquenceur automatique (ABI 3730, Applied Biosystems) et analyse des données avec le logiciel SeqScape v2.6. Recherche de réarrangements exoniques (délétions ou multiplications) par dosage semi-quantitatif (kits SALSA MLPA Parkinson, P051/P052, MRC Holland) puis analyse des données par le logiciel GeneMapper v4.0. Résultats : - Parmi les 203 cas familiaux, 65 familles ont au moins une mutation dans la Parkine ( 5 sont hétérozygotes et 60 ont 2 mutations dont 1 famille avec aussi la mutation G2019S dans LRRK2), 7 ont 2 mutations dans PINK1, aucune famille n'a de mutation dans DJ-1, 8 sont hétérozygotes pour la mutation G2019S et 1 famille porte une duplication de SNCA. - Parmi les 345 cas isolés, 51 familles ont au moins une mutation dans la Parkine (17 sont hétérozygotes et 34 ont 2 mutations dont 2 cas avec aussi la mutation G2019S dans LRRK2), 1 cas porte 2 mutations dans PINK1, aucun cas n'a de mutation dans DJ-1, 12 sont hétérozygotes pour la mutation G2019S et 1 cas porte une duplication de SNCA. Conclusion: Dans cette étude, les mutations dans les gènes associés à la MP représentent $\sim 40 \%$ des formes familiales précoces et $\sim 20 \%$ des cas isolés atteints de la MP.

Mots-clés : Parkinson, âge de début précoce, gènes PARK.

\section{- P454. ARBRES DÉCISIONNELS POUR LES ANALYSES DE GÉNÉTIQUE MOLÉCULAIRE DES FORMES FAMILIALES DE} LA MALADIE DE PARKINSON

Réseau des Laboratoires de Diagnostic Moléculaire des Maladies Génétiques Neurologiques, Musculaires, Neurosensorielles et Retards Mentaux, C. Sân (1), A. Dürr (2, 3, 4), E. Leguern $(1,2)$, C. Cazeneuve (1)

(1) Unité Fonctionnelle de Neurogénétique Moléculaire et Cellulaire, Département de Génétique et Cytogénétique, Groupe Hospitalier PitiéSalpêtrière, Assistance Publique - Hôpitaux de Paris, Paris, France ; (2) Inserm UMR_S975, Groupe Hospitalier Pitié-Salpêtrière, Paris, France, (3) Centre de Référence des Maladies Neurogénétiques, Groupe Hospitalier Pitié-Salpêtrière, Assistance Publique - Hôpitaux de Paris, Paris, France; (4) Unité Fonctionnelle de Génétique Clinique, Département de Génétique et Cytogénétique, Groupe Hospitalier Pitié-Salpêtrière, Assistance Publique - Hôpitaux de Paris, Paris, France

Contact : cecile.cazeneuve@psl.aphp.fr

L'élaboration d'arbres décisionnels pour les analyses de génétique moléculaire et pour les prérequis cliniques amenant à la prescription de ces 
analyses constitue une démarche indispensable pour les laboratoires de génétique moléculaire afin d'améliorer la qualité du service rendu tout en tenant compte de la limitation des moyens humains et des moyens de fonctionnement dont ils disposent. Un des objectifs du réseau des laboratoires de diagnostic moléculaire des maladies génétiques neurologiques, musculaires, neurosensorielles et retards mentaux est de rédiger ces arbres décisionnels, action favorisée pour les aspects analytiques par le rapprochement des acteurs des différents laboratoires de génétique moléculaire et, pour l'établissement des prérequis cliniques, par une collaboration avec les centres de référence maladies rares. La maladie de Parkinson est caractérisée cliniquement par la triade bradykinésie, rigidité, tremblement de repos, dus à la perte des neurones dopaminergiques de la substance noire. La réponse positive à un traitement par L-Dopa est un critère essentiel. Touchant environ $1 \%$ de la population de plus de 60 ans, c'est la plus fréquente des maladies neurodégénératives associées à des mouvements anormaux. La très grande majorité des cas est sporadique. Les formes familiales de la maladie, autosomiques récessives ou dominantes, représentent 5 à $15 \%$ des cas, généralement associées à un âge de début plus précoce. Actuellement 8 gènes ont été identifiés dont 4 responsables de formes autosomiques dominantes. Compte tenu du grand nombre de personnes atteintes et de l'hétérogénéité génétique, l'établissement de prérequis cliniques pour la prescription des analyses de génétique moléculaire est particulièrement critique. Compte tenu des données cliniques et des résultats des études moléculaires princeps, des critères de décisions simples, basés sur le mode de transmission, sur l'âge de début et sur l'origine géographique des patients, ont permis d'élaborer des prérequis cliniques pour la demande d'analyses génétiques. De même, la pathologie moléculaire des gènes impliqués a conduit à l'élaboration de stratégies diagnostiques. La confrontation de ces arbres décisionnels avec l'expérience d'un laboratoire ayant testé plus de 350 patients pour les gènes PARK2, SNCA ou LRRK2 a permis leur validation a posteriori.

Mots-clés : arbre décisionnel, Parkinson, critères cliniques.

\section{- 455 . DIAGNOSTIC MOLÉCULAIRE DES FORMES HÉRÉDI- TAIRES DE LA MALADIE DE PARKINSON}

C. Cazeneuve (1), C. Sân (1), E. Leguern (1, 2), A. Dürr (2, 3), A. Brice $(2,3)$, et le groupe Parkinson Disease Genetics

(1) Unité Fonctionnelle de Neurogénétique Moléculaire et Cellulaire, Département de Génétique et Cytogénétique, Groupe Hospitalier PitiéSalpêtrière, Assistance Publique - Hôpitaux de Paris, Paris, France ; (2) Inserm UMR_S975, Groupe Hospitalier Pitié-Salpêtrière, Paris, France ; (3) Unité Fonctionnelle de Génétique Clinique, Département de Génétique et Cytogénétique, Groupe Hospitalier Pitié-Salpêtrière, Assistance Publique - Hôpitaux de Paris, Paris, France

Contact : cecile.cazeneuve@psl.aphp.fr

La maladie de Parkinson est caractérisée par la triade bradykinésie, rigidité, tremblement de repos et par une bonne sensibilité à la L-Dopa. Touchant environ $1 \%$ de la population de plus de 60 ans, c'est la plus fréquente des maladies neurodégénératives associées à des mouvements anormaux. La grande majorité des cas est sporadique. Les formes familiales de la maladie représentent 5 à $15 \%$ des cas, souvent associées à un âge de début précoce. Huit gènes ont été identifiés dont 4 responsables de formes autosomiques dominantes, dont les gènes LRRK2 et SNCA. Le gène PARK2 est le plus fréquemment en cause dans la forme juvénile autosomique récessive de la maladie de Parkinson. Nous présentons ici le résultat de l'analyse de 363 familles ( 1 ou plusieurs atteints par famille) ayant un diagnostic clinique de maladie de Parkinson et adressées pour étude du gène PARK2 (229 familles) et/ou des gènes SNCA et/ou LRRK2 (134 familles). La recherche de réarrangement (gènes PARK2 et SNCA) a été réalisée par Multiplex Ligation-dependent Probe Amplification (MLPA kits P051/P052, MRC-Holland) ; les mutations ponctuelles du gène PARK2 et la mutation p.Gly2019Ser du gène LRRK2 ont été recherchées par séquençage. Au total, 2 mutations de PARK2 ont été identifiées dans $46 \%$ (13/28) des familles avec transmission autosomique récessive; le pourcentage chute à $13 \%(17 / 128)$ pour les cas sporadiques avec un âge de début $\leq 40$ ans ; un seul patient cas isolé avec un âge de début à 45 ans porte 2 mutations. Les réarrangements représentent $59 \%(40 / 68)$ des allèles indépendant mutés; les mutations ponctuelles sont des mutations faux-sens $(\mathrm{n}=10)$, frameshift $(\mathrm{n}=16)$, non-sens $(\mathrm{n}=1)$ ou conduisant à l'insertion d'un acide aminé $(\mathrm{n}=1)$. Parmi les porteurs de 2 mutations, l'âge de début ne differe pas significativement selon que les patients portent 0,1 ou 2 réarrangements ou selon le nombre d'allèle muté conduisant a priori à une protéine tronquée. Dix patients portent une seule mutation identifiée, dont 2 portent également la mutation p.Gly2019Ser (gène LRRK2). L'âge de début moyen chez les porteurs de 2 mutations est inférieur à celui des
SNCA a été étudié dans 65 familles et la seule mutation p.Gly2019Ser du gène LRRK2 a été recherchée dans 69 familles. Une duplication du gène SNCA a été identifiée chez 2 patients, dont l'un se présente comme un cas isolé. La mutation p.Gly2019Ser a été identifiée dans 31 familles, dont une fois à l'état homozygote, 26 de ces familles étant originaires du Maghreb. Une nouvelle mutation du gène LRRK2 (c.5957A $>$ C - p.His1986Pro) a été identifiée dans une famille dominante caucasienne. Au total, ces résultats montrent la nécessité de suivre les arbres décisionnels établis pour les analyses de génétique moléculaire des formes familiales de la maladie de Parkinson et élaborés conjointement par les centres de référence maladies rares et les laboratoires de génétique moléculaire.

Mots-clés : Parkinson, diagnostic moléculaire, critères cliniques. -P456. MALADIE DES GANGLIONS DE LA BASE SENSIBLE À
LA BIOTINE : IDENTIFICATION DE DEUX NOUVELLES MUTA-
TIONS DE SLC19A3 DANS UNE FAMILLE PORTUGAISE

A. Rastetter (2, 3), C. Depienne (2, 3, 4), R. Debs (1, 2), C. Jardel (4), B. Keren (4), A. Brice $(2,3,4)$, F. Sedel $(1,2)$

(1) Fédération des maladies du système nerveux, Assistance PubliqueHôpitaux de Paris, Hôpital de la Salpêtrière, Paris, France ; (2) Université Pierre-et-Marie-Curie6, Paris, France : (3) INSERM UMR_S975 (ExU679), Paris, France; (4) AP-HP, Département de génétique et cytogénétique, Fédération de Génétique, Hôpital de la Salpêtrière, Paris, France Contact : christel.depienne@upmc.fr

La maladie des ganglions de la base répondant à la biotine est une maladie autosomique récessive rare qui a été décrite en 1998 dans huit familles Saoudienne, syriennes et yéménites. La maladie se présente avec des épisodes d'encéphalopathie aiguë qui peut conduire à une invalidité permanente (motrice et cognitive), voire au coma et à la mort si elle reste non traitée. Entre ces épisodes, prédominent des symptômes extrapyramidaux. L'IRM cérébrale présente spécifiquement des lésions bilatérales de la tête du noyau caudé, et perte partielle ou complète du putamen. L'administration de fortes doses de biotine conduit à une amélioration partielle ou totale des symptômes extrapyramidaux en quelques jours ou quelques mois, tandis que l'interruption du traitement à la biotine conduit à la réapparition des symptômes. En 2005, le gène causal a été cartographié en $2 \mathrm{q} 36.3$ et deux mutations faux-sens ont été identifiées dans le gène SLC19A3, codant pour hTHTR2, deuxième protéine transporteur de la thiamine. Toutefois, la biotine ne semble pas être un substrat de hTHTR2 et l'interaction entre la biotine et ce transporteur de thiamine ainsi que le mécanisme précis par lequel la biotine rétablit, au moins partiellement, le phénotype clinique demeurent inconnues. Jusqu'à présent, la maladie n'avait jamais été observée en dehors du Moyen-Orient. Dans cette étude, nous rapportons une famille portugaise avec deux patients adultes présentant des symptômes évocateurs de la maladie des ganglions de la base sensible à la biotine. Le premier patient a répondu à une dose élevée de biotine, alors que pour le second, l'ajout de thiamine a été nécessaire, suggérant ainsi un rôle majeur du transporteur de la thiamine dans la physiopathologie de cette maladie. Le séquençage du gène SLC19A3 chez ces patients à partir d'ADN génomique extrait de lymphocytes du sang périphérique a permis d'identifier deux nouvelles mutations hétérozygotes composites. La première est la duplication d'une thymine dans l'exon 2 (c.74dupT/ p. Ser26LeufsX19) entraînant un décalage du cadre de lecture et créant un codon stop prématuré. La seconde est la substitution intronique d'un nucléotide, 14 bases en amont de l'exon 4 (c.980-14 A>G) créant un nouveau site accepteur d'épissage. L'étude de l'ARN messager extrait à partir de fibroblastes a ainsi montré que la mutation intronique conduit à un saut de l'exon 4. Ces résultats montrent que cette maladie est également présente en Europe et suggère que les mutations dans $\mathrm{SLC1} 19 \mathrm{~A} 3$ causent une perte de fonction de la protéine hTHTR2.

Mots-clés : SLC19A3, biotine sensible, maladie métabolique.

\section{EP457. NEURO-DÉGÉNÉRESCENCE AVEC ACCUMULATION DE FER DANS LE CERVEAU : UNE ÉTUDE GÉNÉTIQUE DE 6 FAMILLES}

E. Mundwiller (1), S. Assami (2), A. Dürr (1, 3), H. Azzedine (1), S. Mahoiu (2), S. Belardi (2), A. Brice (1, 3), M. Tazir (2), G. Stevanin (1, 3)

(1) INSERM/UPMC UMR_S975, CNRS 7225, Centre de Recherche de l'Institut du Cerveau et de la Moelle Épinière, NEB, GHU Pitié-Salpêtrière, Paris, France ; (2) Département de Neurologie, CHU MUSTAPHA, Alger, Algérie : (3) APHP, Dpt de Génétique et Cytogénétique, GHU PitiéSalpêtrière, Paris, France

Contact : emeline.mundwiller@upmc.fr

Objectifs : Notre étude a consisté en la recherche de mutations et de réarrangements dans le gène codant pour la pantothénate kinase 2 (PANK2) 
dans 6 familles présentant des signes caractéristiques d'une neuro-dégénérescence avec accumulation de fer dans le cerveau. Introduction : Il s'agit d'une maladie neuro-dégénérative rare à hérédité autosomique récessive qui se déclare dans l'enfance. Elle est caractérisée principalement par une dystonie progressive et une accumulation de fer dans les noyaux gris. Cette accumulation se traduit sur l'IRM cérébrale par un hyposignal T2 du pallidum associé à un hypersignal T2 du pallidum interne avec l'aspect caractéristique en " œil de tigre ». Plusieurs études ont montré la liaison entre cet aspect d' « œil de tigre » et le gène codant pour la pantothénate kinase 2 (PANK2) localisé sur le chromosome 20p13. Cette enzyme mitochondriale est un régulateur clé dans la voie de synthèse du Coenzyme A. Un déficit en PANK2 entraine une accumulation de cystéine qui chélate le fer traduisant cet aspect typique à l'IRM. Méthodes: Séquençage et recherche de réarrangements par la technique de MLPA dans le gène PANK2 dans 6 familles dont 4 avec un phénotype typique. L'IRM n'était pas disponible pour les patients des 2 autres familles. Résultats : Les 6 familles étudiées présentent des mutations dans le gène PANK2. Deux mutations tronquantes homozygotes ont été identifiées dans 2 familles d'origine algérienne. Une des ces 2 mutations a été retrouvée à l'état hétérozygote dans une troisième famille algérienne co-ségrégeant avec 2 variations faux-sens absentes chez des contrôles caucasiens et nord-africains. Les effets délétères de ces 2 variations faux-sens restent à déterminer. Dans les 3 autres familles d'origine française, 1 mutation tronquante hétérozygote et 4 variations fauxsens hétérozygotes ont été observées. Ces dernières sont absentes chez des contrôles caucasiens et concernent des acides aminés conservées au cours de l'évolution. Parmi ces 4 variations faux-sens, une (p.G521R) a déjà été impliquée dans plusieurs familles et est associée à une diminution d'activité de l'enzyme. Conclusions et perspectives: Tous les patients atteints des 6 familles analysées s'avèrent mutés dans le gène PANK2. Parmi les familles pour lesquelles l'IRM était disponible, nous confirmons le lien entre la présence d'un « œil de tigre» et des mutations dans PANK2.

Mots-clés : neuro-dégénérescence, œil de tigre, PANK2.

\section{IP458. DÉFICIT EN GLUT1 (MALADIE DE VIVO) : 23 CAS DIA- GNOSTIQUES EN FRANCE}

S. Vuillaumier-Barrot $(1,2)$, C. Le Bizec (1), T. Dupré (1, 2), G. Louis (1), N. Bahi-Buisson (3), S. Odent (4), M. Mayer (5), D. Chaigne (6), S. Bekri (7), V. Drouin-Garraud (8), A. Goldenberg (8), E. Flori (9), E. Raffo (10), J. Mancini (11), S. Julia (12), A. Roubertie (13), A. Roubergue (14), D. Doummar (15), K. Maincent (15), J.M. Pedespan (16), V. Des Portes (17), M.A. Cournelle (18), A. Le Bihannic (19), D. Heron (20), F. Dubois-Teklali (21), P. De Lonlay (22), N. Seta (1)

(1) AP-HP, Biochimie, Hôpital Bichat, Paris, France ; (2) INSERM U773 CRB3, Paris, France ; (3) APHP, Hôpital Necker, Neuropédiatrie, Paris, France; (4) Génétique Médicale, CHU de Rennes, France; (5) APHP, Hôpital Saint-Vincent-de-Paul, Neuropédiatrie, Paris, France; (6) Neuropédiatrie, CH Marie Madeleine, Forbach, France ; (7) Biochimie Médicale, CHU de Rouen, France; (8) Génétique, CHU de Rouen, France; (9) Génétique Médicale, Hôpital de Hautepierre, Strasbourg, France ; (10) Medecine infantiles et Génétique Médicale, CHU de Nancy, France; (1I) Neurologie Pédiatrique, CHRU de Marseille, France; (12) Génétique Médicale, Hôpital Purpan, CHU de Toulouse, France ; (13) Neuropédiatrie, CHRU de Montpellier, France; (14) Neurologie, Hôpital Saint Antoine, France ; (15) Neuropédiatrie, Hôpital Trousseau, France ; (16) Pédiatrie, CHU de Bordeaux, France; (17) Neuropédiatrie, Hôpitaux de Lyon, France; (18) Pediatrie, Centre hospitalier du pays d'Aix, France; (19) Pédiatrie, Centre Hospitalier de Saint-Brieuc, France; (20) APHP, Groupe Hospitalier Pitié-Salpêtrière, Centre de Référence "Déficiences intellectuelles " Département de Génétique et Cytogénétique, Paris, France; (21) CHU de Grenoble, Neuropédiatrie, France; (22) APHP, Hôpital Necker, Maladies Métaboliques, Paris, France

Contact : sandrine.vuillaumier@bch.aphp.fr

Le syndrome de déficit en transporteur du glucose de type 1 (GLUT-1, OMIM 606777), décrit en 1991 par De Vivo, de transmission autosomique dominante, est lié à un défaut de transport du glucose au niveau de la barrière hémato-encéphalique (environ 100 cas dans le monde publiés). Il se manifeste par une encéphalopathie comportant une épilepsie pharmacorésistante, une microcéphalie acquise, un retard du développement psychomoteur, une ataxie intermittente et d'autres troubles neurologiques paroxystiques (mouvements anormaux). Le diagnostic, orienté par la clinique et une hypoglycorachie (glycorachie/glycémie $<0,4$ ), repose sur la mise en évidence d'une mutation hétérozygote sur le gène SLC2A1, le plus souvent de novo. La recherche de mutations sur SCL2A1 se fait par séquençage des 10 exons et des régions introniques flanquantes, puis, en cas d'absence de mutation, par la recherche d'une hémizygotie par PCR en temps réel et par MLPA (MRC-Holland). La causalité des mutations faux-sens est vérifiée par un test phénotypique de captation par les globules rouges de déoxy-D-glucose marqué au carbone 14. Au cours des 4 dernières années, 65 demandes de diagnostic nous ont été adressées, parmi lesquelles nous avons mis en évidence une mutation dans 23 cas (35\% des cas étudiés) (âge médian de diagnostic : 9 ans) dont 17 sont nouvellement décrites: 6 décalages du cadre de lecture [c.737_740del AAGA ; c.32_33del GC; c.970insA(+)c.970_972+3delTCGGTG; c.21delG (p.Lys7AsnfsX2); c.1365dupT], 1 petite délétion [c.493_504Del12 (p.Val165Ile168Del)], 1 mutation d'épissage [c.1257C> T (p.Gly419AlafsX28)] et 4 mutation non-sens [c.338C $>$ A (p.Ser113X); c.143G > A (p.trp48X); c.121G> T (p.Glu41X) ; c.349A > T p.Lys117X]. Enfin, les 6 faux-sens [c.68C $>$ T (p.Ser23Phe) ; c.409A $>$ G (p.Thr127Ala); c.388G > A (p.Gly130Ser); c.443C $>\mathrm{T}$ (p.Ser148Leu); c.425T $>\mathrm{A}$ p.Met142Lys ; c.94G >C p.Val34Leu] sont données comme pathogènes par l'analyse in silico. Les 5 mutations restantes [c.436G> A ; p.Glu146Lys ; c. $884 \mathrm{C}>\mathrm{T}$ p. Thr 295 Met; c. $376 \mathrm{C}>\mathrm{T}$ p.Arg126Cys; c. $988 \mathrm{C}>\mathrm{T}$ p.Arg $330 \mathrm{X}]$ et une délétion du gène entier retrouvée chez 2 patients (en cours de bornage par puce CGH Agilent $15 \mathrm{~K}$ ) avaient été décrites précédemment. L'exon 4 semble particulièrement impliqué ( $9 / 23$ mutations). Trois cas sont familiaux où les 3 parents sont peu symptomatiques (épilepsie isolée dans l'enfance). Le test phénotypique, diminué de $50 \%$ chez une famille porteuse d'une délétion de 4 acides aminés (père et enfant), a permis de confirmer le caractère pathogène de la mutation. Dans notre série, les signes cliniques sont dominés par l'épilepsie ( $87 \%)$, les troubles moteurs $(70 \%)$, et un léger retard mental plus important en présence de mutations sévères. Le diagnostic de déficit GLUT1, certainement sousdiagnostiqué ou encore tardivement évoqué, doit être plus souvent demandé, d'autant qu'il existe une possibilité de traitement par un régime cétogène.

Mots-clés : GLUT1, de vivo, épilepsie.

EP459. HYPEREXPLEXIA : ASPECTS CLINIQUES ET GÉNÉTIQUES D'UNE FAMILLE

V. Barre-Dezellus (1, 3), J.C. Netter (2), B. Tauzin (2), S. Marret (3) J.F. Vanbellinghen (4)

(1) Service de pédiatrie, Centre hospitalier du Belvédère, Mont-SaintAignan, France ; (2) Service de pédiatrie, Hôpital de Tarbes, France ; (3) Service de médecine néonatale, CHU de Rouen, France; (4) Laboratoire de biologie moléculaire, CHU Sart Tilman, Liège, Belgique

\section{Contact : véronique.barre@ch-belvedere.fr}

Résumé : L'hyperekplexia est une maladie neurologique d'expression néonatale caractérisée par une hypertonie marquée associée à des manifestations paroxystiques non épileptiques déclenchées par des stimuli auditifs, visuels, ou des manipulations. Le risque de mort subite est réel la première année, l'hypertonie axiale disparait en 2 à 3 ans mais des anomalies du psychomotrices persistent toute la vie. Nous rapportons l'observation de Damien présentant dés la naissance une hypertonie majeure disparaissant lors du sommeil et une hyperexcitabilité avec difficultés d'alimentation faisant suspecter un syndrome de sevrage aux morphiniques. Les difficultés d'alimentation avec pleurs incessants vont être compliquées d'une exceptionnelle hernie ombilicale étranglée et de hernies inguinales. L'hypertonie sera majeure jusqu'a l'âge de 2 ans associée à des accès de panique lors de certains bruits et des troubles du sommeil. À 6 ans, l'enfant a une intelligence normale mais une rééducation orthophonique pour dysarthrie et une rééducation en psychomotricité pour une dyspraxie gênant l'apprentissage de l'écriture. Il présente des impériosités mictionnelles nécessitant un traitement et un aménagement scolaire. Sa maman présentait des pieds bots varus équins à la naissance et n'a marché qu'à l'âge de 7 ans. Elle a été hospitalisée de façon récurrente pour des problèmes neurologiques mal expliqués jusqu'à l'âge de 3 ans. Elle a deux premiers enfants bien portants. Elle prépare un diplôme d'aide soignante. Sacha est le cousin germain de Damien. Il est hospitalisé à $\mathrm{j} 3$ pour suspicion de convulsions, présente des accès d'hypertonie en opisthotonos et des sursauts à la moindre stimulation. À un an, son tonus est typique du stiffbaby syndrome, il présente des apnées du sommeil. L'étude génétique a mis en évidence une substitution c $838>\mathrm{A}$ au niveau de l'exon 7 du gène GLRA1 chez Damien et sa mère et chez Sacha et sa mère, sœur de la maman de Damien. Le gène GLRAlest localisé en 5q33-35. Les mutations du gène GLRAl (dominante ou récessive) et du gène SLCGA5 ne permettent d'expliquer que 30 à $40 \%$ des cas d'hyperekplexia Cette affection probablement sous-diagnostiquée du fait de l'hétérogénéité clinique mérite d'être mieux reconnue pour une prise en charge appropriée.

Mots-clés : hyperexplexia, neonate, biologie moléculaire. 
[P460. ÉTUDE DES DÉTERMINANTS GÉNÉTIQUES DES ENCÉPHALOPATHIES ÉPILEPTIQUES PRÉCOCES : UNE MALADIE DE LA NEUROTRANSMISSION ?

M. Milh (1, 2, 3), C. Mignon-Ravix (2, 3), P. Cacciagli (2, 3), B. El-Waly $(2,3)$, B. Chabrol $(1,2,3)$, L. Villard $(2,3)$

(1) Service de Neurologie Pédiatrique, Hôpital d'Enfants de La Timone, Marseille, France ; (2) INSERM UMR-S 910, Marseille, France ; (3) Université de la Méditerranée, Marseille, France

Contact : mathieu.milh@ap-hm.fr

Les encéphalopathies épileptiques précoce (EEP) constituent un groupe hétérogène de maladies du développement, dont les points communs sont : - une épilepsie sévère débutant dans les trois premiers mois de vie, avec des crises multifocales, des spasmes épileptiques et/ou des myoclonies ; un tracé EEG très perturbé, avec des anomalies multifocales et une altération du rythme de fond pouvant aller jusqu'au tracé de type «suppression-burst n- un trouble sévère du développement, avec un pronostic vital parfois mis en jeu à court terme, et dans tous les cas un polyhandicap sévère. Les EEP peuvent être en rapport avec des anomalies morphologiques cérébrales (Lissencéphalie, hémimégalencéphalie...), ou des anomalies fonctionnelles, avec une IRM cérébrale normale. Nous nous intéressons à ce dernier groupe de sujet, et basée sur la réalisation d'un modèle animal d'EEP, nous faisons l'hypothèse qu'il s'agit d'un trouble sévère de la neurotransmission glutamatergique. Notre projet consiste à étudier tous les gènes impliqués dans la neurotransmission glutamatergique chez les patients EEP. Cette hypothèse a été renforcée par la découverte récente d'un gène impliqué dans les EEP ; le gène STXBP1/MUNC18-1, impliqué dans la libération vésiculaire des neurotransmetteurs. Nous avons débuté une étude multicentrique visant à collecter de manière prospective les nouveaux cas d'EEP avec IRM normale, dont le bilan étiologique est négatif. Notre étude comportera ensuite trois étapes: 1) Séquençage des gènes impliqués dans les EEP : STXBP1/MUNC18-1 et ARX chez le garçon. 2) Screening par puce CGH $244 \mathrm{~K} 3$ ) Screening par puce dédiée haute-résolution de 2205 exons appartenants à des gènes impliqués dans le métabolisme du glutamate. Les résultats préliminaires de ce travail seront présentés lors des Assises.

Mots-clés : épilepsies, encéphalopathie, neurotransmission.

\section{- P461. STRATÉGIE DIAGNOSTIQUE ACTUELLE DANS LES CÉROÏDE-LIPOFUSCINOSES}

J.P. Puech (1), J. Dussau (1), A. Gelot (3, 2), B. Chabrol (4), C. Caillaud $(1,2)$

(1) Laboratoire de Biochimie Génétique (AP-HP), Faculté de Médecine Cochin Port-Royal, Paris, France ; (2) Institut Cochin, INSERM U567, CNRS UMR 8104, Université Paris Descartes, Paris, France; (3) Unité de Neuropathologie, Hôpital Armand-Trousseau, Paris, France ; (4) Unité de Médecine Infantile, Hôpital Timone Enfants, Marseille, France Contact : catherine.caillaud@inserm.fr

Les céroïde-lipofuscinoses neuronales (CLN) sont des maladies neurodégénératives, à transmission récessive autosomique, caractérisées par l'accumulation intralysosomale de lipopigments autofluorescents, notamment dans les neurones. Quatre formes cliniques principales ont été décrites: infantile (débutant entre 6 et 18 mois), infantile tardive (entre 2 et 4 ans), juvénile (entre 4 et 7 ans) et adulte. Cependant, de très nombreux variants ont été rapportés, laissant présager une grande hétérogénéité génétique. À ce jour, huit loci ont déjà été impliqués dans la genèse de ces affections. Le gène CLN1 code la palmitoyl protéine thioestérase (PPT) et le gène CLN2 la tripeptidyl peptidase I (TPP-I). Six autres gènes codant pour des protéines transmembranaires ont été rapportés : CLN3 (variant juvénile), CLN5 (variant finlandais), CLN6 (variant indo-européen), CLN8 (Northern epilepsy), CLN10 (cathepsine D) et plus récemment CLN7 (ou MFSD8). Des patients français ou originaires d'Afrique du nord suspects de CLN ont été étudiés pour ces différents loci. Une dizaine d'enfants atteints de forme infantile de la maladie ont pu être caractérisés à la fois par dosage de la PPT et mise en évidence de mutations sur le gène CLN1. En ce qui concerne la forme juvénile classique de la maladie, la plupart des patients français testés pour le gène CLN3 étaient porteurs de la délétion commune de $1,02 \mathrm{~kb}$. Dans la forme infantile tardive, la plus fréquente en France, le gène CLN2 a été trouvé impliqué chez quarante quatre patients. La moitié d'entre eux étaient porteurs des mutations communes R208X et IVS5G>C. Chez les autres patients, de nombreuses anomalies nouvelles de type délétions, insertions, mutations non sens ou faux sens ont pu être mises en évidence. Pour ces dernières, un travail de modélisation est en cours pour confirmer leur vraisemblable caractère délétère. Chez les autres patients présentant une forme infantile tardive, l'implication des gènes CLN5, CLN8 et CLN10 a pu être exclue par séquençage direct.
CLN6. Il étaient principalement originaires du Pakistan ou d'Afrique du Nord. Enfin, tout récemment, des mutations du gène CLN7 ont été retrouvées chez deux patients ayant un phénotype clinique très similaire aux patients CLN2. Au total, dans les formes infantiles tardives, la stratégie diagnostique actuelle débute par l'étude des gènes CLN2 et CLN7 respectivement par dosage enzymatique et séquençage direct. Par la suite, un examen neuropathologique est souhaitable afin de confirmer le diagnostic de CLN (surcharge curvilinéaire, granulaire, empreinte) avant d'entreprendre le séquençage des gènes alternatifs (connus ou candidats). Pour les formes infantiles et juvéniles, le diagnostic peut généralement être assuré par l'étude des gènes CLN1 et CLN3. Enfin, les formes adultes et congénitales restent encore à décrypter.

Mots-clés : céroöde-lipofuscinoses, palmitoyl protéine thioestérase, tripeptidyl peptidase I.

aP462. HYPOPLASIE PONTOCÉRÉBELLEUSE ASSOCIÉE À DES ANOMALIES DU GËNE CASK : CONFIRMATION D'UN PHÉNOTYPE RECONNAISSABLE ET 1ER CAS DE MOSAÏQUE SOMATIQUE

L. Burglen (1, 8), S. Chantot-Bastaraud (1, 8), A. Afenjar $(2,8,9)$, C. Goizet (3), M. Milh (4), N. Philip (5), A. Legall (1), N. Jeanne (1), I. Desguerre (6), D. Lacombe (3), C. Garel (7, 8), D. Rodriguez $(2,8)$ (1) Service de Génétique Médicale, AP-HP, Hôpital Trousseau, Paris, France ; (2) Service de Neuropédiatrie, AP-HP, Hôpital Trousseau, Paris, France; (3) Service de Génétique Médicale, Centre Hospitalier Universitaire, Bordeaux, France: (4) Service de Neuropédiatrie, Hôpital La Timone Enfants, Marseille, France ; (5) Service de Génétique, Hôpital La Timone Enfants, Marseille, France; (6) Service de Neuropédiatrie, AP-HP, Hôpital Necker-Enfants Malades, Paris, France ; (7) Service de Radiologie, AP-HP, Hôpital Trousseau, Paris, France ; (8) Centre de référence des malformations et maladies congénitales du cervelet, $A P-H P$, Hôpital Trousseau, Paris, France ; (9) Département de génétique, AP-HP, Pitié Salpétrière, Paris, France

Contact : lydie.burglen@trs.aphp.fr

Les hypoplasies pontocérébelleuses (HPC) sont des pathologies le plus souvent autosomiques récessives, caractérisées par une insuffisance de développement et/ou une neurodégénérescence précoce du cervelet et du tronc cérébral. On en distingue cliniquement 5 sous-types: HPC2, le plus fréquent, avec microcéphalie progressive et dystonie-choréoathétose liée à des mutations de 3 des 4 sous-unités du complexe tRNA splicing endonuclease; HPC4, forme sévère d'HPC2; $\mathrm{HPCl}$ avec atteinte de la corne antérieure de la moëlle ; HPC 3 et 5 entités très rares. Enfin, des délétions et des mutations de novo du gène CASK situé en Xp22, codant pour une protéine intervenant dans la régulation de l'expression de la Reelin, ont été identifiées chez des filles présentant une HPC avec microcéphalie et chez un garçon atteint d'une forme sévère. Nous rapportons 7 patients ( 6 filles et un garçon ; âge 2-16 ans) présentant une HPC ne répondant pas aux critères des HPC 1, 2 et 4 (absence d'atteinte de la corme antérieure et de mouvements choréoathétosiques, retard des acquisitions moins sévère) chez lesquels des anomalies de novo du gène CASK ont été identifiées (mutations non-sens et d'épissage hétérozygotes $(6 / 7)$, large délétion Xp22 de $3 \mathrm{Mb}(1 / 7))$. L'analyse du phénotype met en évidence un tableau reconnaissable : hypotonie et retard moteur constants avec une marche non acquise ou acquise éventuellement après 3 ans; dystonie et mouvements bucco-linguaux mais sans mouvements choréathétosiques ; langage absent ou réduit à quelques mots ; microcéphalie postnatale sévère $(-4,5$ à $-6 \mathrm{DS}$ ) ; épilepsie peu fréquente (2/6); atteinte ophtalmologique (mégalocornée, glaucome, papilles pâles, rétine poivre et sel) ; déficit auditif (3/6); scoliose : anomalies mineures des extrémités et dysmorphie (visage rond, sourcils en arc de cercle, racine du nez haute, lèvre inférieure charnue); retard statural inconstant. L'imagerie cérébrale retrouve dans tous les cas une hypoplasie du vermis et des hémisphères cérébelleux de sévérité variable, ainsi qu'une hypoplasie du tronc cérébral. L'unique garçon de notre série, contrairement au patient décrit dans la littérature et à ce qui est attendu pour une pathologie liée à l'X, présente un phénotype moins sévère: marche acquise à 3 ans, non ataxique, langage avec petites phrases, microcéphalie (-3DS), absence de signes neurologiques. L'imagerie cérébrale, qui a été l'élément orientant vers le gène CASK est en revanche peu différente de celle des autres patientes. De façon intéressante, il a été retrouvé chez ce patient une mutation non-sens en mosaïque dans les lymphocytes. En conclusion, l'étude du gène CASK est indiquée chez tout enfant, fille mais aussi garçon, présentant un tableau radiologique d'HPC associé à une microcéphalie progressive sévère, un retard psychomoteur avec dystonie et en l'absence de signe orientant vers une HPC de type 1 (atteinte de la corme antérieure) ou 2/4 (mouvements choréoathétosiques et quasi absence d'acquisitions).

Mots-clés: hypoplasie pontocérébelleuse, gène CASK, mosaique somatique. 
IP463. ENCÉPHALITE AIGUË NÉCROSANTE FAMILIALE ET MUTATION DU GENNE RANBP2

E. Purenne (1), D. Rodriguez (2, 4), L. Balu (1), L. Burglen $(3,4)$ (1) Service de réanimation pédiatrique, AP-HP, Hôpital Bicêtre, Le Kremlin-Bicêtre, France; (2) Service de Neuropédiatrie, AP-HP, Hôpital Trousseau, Paris, France; (3) Service de Génétique Médicale, AP-HP, Hôpital Trousseau, Paris, France; (4) Centre de référence des maladies neurogénétiques, Pitié-Trousseau, Paris, France

Contact : lydie.burglen@trs.aphp.fr

Les encéphalites aigues nécrosantes (ANE) sont des encéphalopathies survenant de façon aiguë au décours d'une infection virale, le plus souvent à virus influenzae ou parainfluenzae. La plupart des ANE surviennent de façon sporadique et ne récidivent pas. Des formes familiales et/ou récurrentes d'ANE ont toutefois été rapportées. Les patients, sans antécédent personnel notable présentent à l'occasion d'un épisode infectieux, un tableau neurologique rapidement progressif avec coma et souvent crises épileptiques et lésions des noyaux gris, des thalami et du tronc cérébral à l'imagerie cérébrale. L'évolution est variable se faisant vers le décès, la guérison complète ou avec séquelles, avec un risque élevé de récurrence à distance $(50 \%)$. Des antécédents familiaux sont parfois observés et des porteurs obligatoires peuvent ne présenter aucun signe $(40 \%)$. Des mutations dominantes, transmises ou de novo, du gène RANBP2 ont été identifiées dans des cas familiaux et ou récurrents d'ANE. Le gène RANBP2 code pour une protéine localisée à la face cytoplasmique du pore nucléaire qui semble avoir de multiples fonctions dont un rôle dans le transport nucléaire des ribonucléoprotéines virales. Nous rapportons une famille comportant 3 patients ayant présenté une ANE et un adulte asymptomatique, porteur obligatoire. Le cas index est une enfant de 6 ans, hospitalisée en réanimation pour un coma faisant suite à l'installation progressive sur 15 jours de troubles de la marche, d'un syndrome cérébelleux puis de troubles de la conscience avec troubles respiratoires au décours d'un épisode viral. La ponction lombaire était normale mais l'IRM révélait des lésions symétriques massives du tronc cérébral, des thalami et des capsules externes. La mère de l'enfant, âgée de 35 ans présente une déficience intellectuelle légère et un syndrome cérébelleux. Elle a présenté une encéphalite aiguë, à l'âge de 6 mois puis 3 autres épisodes dont le dernier à l'âge de 24 ans. L'interrogatoire du grand-père maternel, totalement asymptomatique, retrouve la notion du décès dans l'enfance d'un demifrère de cette jeune femme. Ce garçon a présenté à l'âge de 1 an, alors que son développement était normal, un tableau neurologique aigu de type encéphalite. Il a évolué avec des séquelles à type de déficience intellectuelle et est décédé à l'âge de 10 ans à l'occasion d'un nouvel épisode d'ANE très brutal. L'étude moléculaire du gène RANBP2 a permis de retrouver chez la petite fille et sa mère la mutation p.Thr $585 \mathrm{Met}$, mutation majoritaire identifiée dans d'autres familles d'ANE et dans des cas sporadiques avec récurrences. En l'absence de données précises sur la prise en charge optimale des patients à risque, se pose dans ces familles la délicate question de l'opportunité du diagnostic présymptomatique.

Mots-clés: encéphalite aiguë nécrosante, gène RANBP2, diagnostic présymptomatique.

IP464. ANALYSE DE SEPT9 DANS LA NÉVRALGIE AMYOTROPHIANTE HÉRÉDITAIRE (NAH)

G. Solé (1, 2), I. Coupry (2), A. Daubos (2), C. Verny (3), E. Ginglinger (4), X. Ferrer (1), D. Lacombe (2, 5), B. Arveiler (2), C. Goizet $(2,5)$

(1) Centre de référence des maladies neuromusculaires et service de neurologie, $\mathrm{CHU}$ de Bordeaux, France; (2) Laboratoire de Génétique Humaine, Université Victor Ségalen Bordeaux 2, France; (3) Centre national de référence pour les maladies neurogénétiques et mitochondriales de l'adulte et département de neurologie, $\mathrm{CHU}$ d'Angers, France : (4) Service de génétique, CHG de Mulhouse, France; (5) Service de génétique, CHU de Bordeaux, France

Contact : guilhem.sole@chu-bordeaux.fr

INTRODUCTION La névralgie amyotrophiante héréditaire (NAH) est responsable d'épisodes de plexopathie douloureuse récidivante essentiellement dans le territoire du membre supérieur. Des mutations de SEPT9 en sont responsables. OBJECTIFS Peu de publications confirment à ce jour l'implication de SEPT9 dans la NAH. L'objectif de notre étude est d'analyser ce gène à la recherche de mutations sur une cohorte de patients français. MÉTHODES Nous avons collecté les prélèvements de 11 patients issus de 6 familles différentes. Après avoir établi la structure introns/exons de SEPT9, nous avons procédé au séquençage direct de ses zones codantes et de certaines zones de régulation. RESULTATS La mutation p.S93F dans l'exon 2 de SEPT9 a été mise en évidence chez un homme de 43 ans ayant présenté au moins deux épisodes authentifiés de NAH, dont le premier à l'âge de 10 ans. Sa mère, actuellement décédée, avait également présenté deux épisodes du même type. DISCUSSION La famille des septines a été initialement identifiée comme un groupe de protéines de régulation du cycle cellulaire, en faisant des cibles thérapeutiques dans les leucémies myéloïdes aigues. Le nombre de cas de NAH liés aux mutations de SEPT9 et rapportés dans la littérature reste faible. La mutation que nous avons mise en évidence était déjà décrite dans une seule famille issue de la publication princeps. Notre étude confirme le rôle pathogène de cette mutation. CONCLUSION Notre étude confirme l'implication de SEPT9 dans la $\mathrm{NAH}$. Nous souhaitons maintenant augmenter la taille de notre cohorte de patients à la recherche de nouvelles mutations.

Mots-clés : SEPT9, névralgie amyotrophiante héréditaire, syndrome de Parsonnage et Turner héréditaire.

\section{UN NOUVEAU LOCUS DANS LA COMPLEXITÉ DES PARAPLÉGIES SPASTIQUES AUTOSOMIQUES RÉCESSIVES} C. Tesson (1), M. Salih (2), P. Coutinho (3), N. Bouslam (1), A.L. Leutenegger (1), E. Denis (4), M. Depla (1), C. Goizet (1), F. Fellman (5), A. Durr $(1,4)$, A. Brice $(1,4)$, G. Stevanin $(1,4)$

(1) INSERM/UPMC UMR_S975, CNRS 7225, Centre de Recherche de l'Institut du Cerveau et de la Moelle Épinière, NEB, GHU Pitié-Salpêtrière, Paris, France; (2) Department of Pediatrics, College of Medicine, Riyadh, Saudi Arabia; (3) Hospital S. Sebastiao, Santa Maria da Feira, Portugal; (4) APHP, Dpt de Génétique et Cytogénétique, GHU PitiéSalpêtrière, Paris, France ; (5) Dpt de Génétique Médicale, CHUV, Lausanne, Suisse

Contact : christelle.tesson@upmc.fr

Les paraplégies spastiques héréditaires (HSP), encore désignées sous le nom de maladie de « Strümpell-Lorrain », constituent un groupe de maladies neurodégénératives rares et très hétérogènes, tant du point de vue clinique que génétique. Le tableau clinique se caractérise par une augmentation des réflexes et une spasticité des membres inférieurs s'aggravant progressivement, et responsables de troubles de la marche et de douleurs. Un tour de génome réalisé sur deux familles consanguines informatives, une d'origine saoudienne et l'autre d'origine portugaise, dans lesquelles se transmet une HSP autosomique récessive nous a permis d'identifier une région d'homozygotie commune de $5 \mathrm{cM}$, située sur le chromosome 4 . Cette région est significativement liée pour chacune des familles avec des LOD-scores multipoints respectivement de 4,8 et 4,6. Dans ces familles les patients $(n=7)$ présentent une forme relativement pure de la maladie débutant très précocement vers l'âge de un an. Afin de réduire la taille de l'intervalle candidat, nous avons analysé, à l'aide de sept marqueurs microsatellites de l'intervalle candidat, 47 familles présentant une HSP se transmettant selon un mode autosomique récessif. Nous avons ainsi pu identifier quatre nouvelles familles liées, dont une famille d'origine saoudienne partageant un haplotype commun avec la famille saoudienne à l'origine de la cartographie. La liaison a été confirmée par le calcul de LOD score puisque la valeur maximum théorique attendue dans cette famille $(Z=2,4)$ est obtenue. Cette famille nous permet de réduire la taille de l'intervalle candidat à une zone de $3,3 \mathrm{cM}$ contenant 24 gènes. Ainsi, nous avons pu identifier un nouveau locus de paraplégie spastique rendant compte de 3 à 6 familles d'origines diverses, ce qui devrait faciliter à terme, l'identification du gène en cause.

Mots-clés : paraplégie spastique, cartographie, hétérogénéité génétique.

DP466. PARAPLÉGIE SPASTIQUE HÉRÉDITAIRE : ORIGINE DE NOVO D'UNE MUTATION DANS LE GENE SPG11

P.S. Denora (1, 2, 5), K. Brockmann (4), M. Ciccolella (5), J. Truchetto $(1,2)$, G. Stevanin $(1,2,3)$, F.M. Santorelli (5)

(1) INSERM, UMR_S975 (formerly U679), Paris, France ; (2) UPMC University Paris 06, UMR_S975, NEB, Centre de Recherche Institut du cerveau et de la Moelle Êpinière, CNRS 7225, Paris, France; (3) APHP, Département de Génétique et Cytogénétique, Groupe Hospitalier Universitaire Pitié-Salpêtrière, Paris, France ; (4) Department of Pediatrics and Neuropediatrics, University of Gottingen, Germany ; (5) Unité de Medicine Moléculaire, IRCCS-Hôpital Pédiatrique Bambino Gesu', Rome, Italie

Contact : palapao@hotmail.it

La paraplégie spastique héréditaire (PSF) associée à un corps calleux fin (Thin Corpus Callosum, TCC) est une forme fréquente et complexe de PSF récessive autosomique, caractérisée principalement par la dégénérescence du faisceau pyramidal et par des signes typiques visibles à l'analyse IRM [TCC et anomalies de la substance blanche (White Matter Abnormalities, WMA)]. La maladie est fréquemment associée à des 
mutations dans le gène SPG11, situé sur le chromosome $15 q$ et codant pour la spatacsine. Notre étude examine le cas d'une patiente de 24 ans qui présente une atteinte motrice progressive avec spasticité et un retard mental associant, à l'IRM, un TCC, des WMA périventriculaires et une atrophie corticale au niveau du lobe frontal. Par séquençage direct du gène, nous avons pu identifier deux mutations tronquantes dans le gène SPG11. L'analyse de ségrégation des mutations dans la famille montre que le père de la patiente est porteur hétérozygote d'un seul des variants, tandis que l'autre variant n'est pas été retrouvé chez d'autres membres de la famille. La reconstitution des haplotypes avec des marqueurs flanquant le gène SPG11 associée à une PCR allèle-spécifique en temps réel et à une PCR-restriction sur différents tissus maternels (lymphocytes, follicules pileux, fibroblastes et cellules épithéliales de l'arbre urinaire) confirment l'origine de novo de la seconde mutation et excluent un mosaïcisme somatique. L'identification de la première mutation de novo met en évidence la complexité et l'importance de l'étude du gène SPG11 en conseil génétique et est en accord avec le fait que la majorité des mutations dans ce gène ont été retrouvées chez des cas sporadiques.

Mots-clés : paraplégie spastique, SPG11, mutation de novo.

P467. LES MUTATIONS PONCTUELLES ET RÉARRANGEMENTS DU GÈNE SPG11/SPATACSINE SONT FRÉQUEMMENT IMPLIQUÉS DANS LES PARAPLÉGIES SPASTIQUES

C. Depienne (1, 2), E. Denis (2), E. Mundwiller (1), E. Fedirko (2), C. Zaros (2), S. Forlani (1), C. Cazeneuve (2), E. Leguern (1, 2), A. Durr $(1,2)$, A. Brice $(1,2), G$. Stevanin $(1,2)$

(1) INSERM/UPMC/NEB, UMR_S975, Bases Moléculaires, Physiopathologie et Traitement des Maladies Neurodégénératives, Centre de Recherche de l'Institut du Cerveau et de la Moelle Épinière, Paris, France ; (2) APHP, Département de Génétique et Cytogénétique, Groupe Hospitalier Pitié-Salpêtrière, Paris, France

Contact : giovanni.stevanin@upmc.fr

Les paraplégies spastiques familiales (PSF) sont des pathologies neurodégénératives génétiquement et cliniquement hétérogènes. Plus de 46 loci sont impliqués dans ces maladies parmi lesquels seulement 19 gènes ont été identifiés à ce jour. Ces affections ont pour point commun une altération de la voie pyramidale (dite cortico-spinale) qui se traduit par une spasticité des membres inférieurs. Au sein des formes récessives, environ $30 \%$ des cas associent des troubles cognitifs ou mentaux et une atrophie du corps calleux à I'IRM. Un gène majeur, SPG11, a été identifié dans ces formes complexes. Nous avons voulu déterminer la fréquence des mutations du gène SPG11 dans le cadre d'une activité diagnostique hospitalière. Ainsi, 60 cas index envoyés au département de génétique et cytogénétique du groupe hospitalier Pitié-Salpêtrière ont été analysés par séquençage direct et par MLPA (multiplex ligation-dependent probe amplification). Nous avons identifié 26 cas index $(43.3 \%)$ porteurs de 40 anomalies génétiques différentes et toutes tronquantes dans le gène SPG11, dont 31 étaient nouvelles. Parmi ces mutations, 35 étaient des mutations ponctuelles ou des délétions/duplications de quelques nucléotides et 5 étaient des réarrangements impliquant un ou plusieurs exons du gène, dont un à l'état homozygote. De plus, 2 mutations faux-sens à l'état hétérozygote composite avec une mutation tronquante et touchant un acide aminé conservé ont également été identifiées dans 2 cas. La ségrégation des mutations chez les autres membres atteints des familles et/ou les parents a été étudiée lorsque des prélèvements étaient disponibles. Ces résultats étendent le spectre mutationnel de SPG11 et soulignent la nécessité d'analyser l'ensemble du gène par séquençage direct. La disponibilité d'un kit de recherche de réarrangements par MLPA permet de rechercher ces évènements, qui représentent $12,5 \%$ des mutations. L'identification et la caractérisation de l'effet pathogène de mutations faux-sens pourrait par ailleurs permettre d'ouvrir des perspectives sur la fonction du gène SPG11 en définissant des domaines fonctionnels.

Mots-clés : paraplégie spastique, SPG11, corps calleux.

DP468. DOMINANT INHERITANCE OF CHARCOT-MARIETOOTH DISEASE WITH GDAP1 MUTATIONS: REPORT OF 11 NEW CASES

N. Bonello (1, 2), N. Martini (1), N. Lévy (1, 2), F. Rejou (3), G. Roche (4), C. Wright (5), C. Goizet (6), P.M. Gonnaud (7), C. Desnuelle (8), L. Leonardis (9), A. Lacour (10), J.M. Faucheux (11), P. Latour (12), R. Bernard $(1,2)$

(1) Département de Génétique Médicale, Hôpital Timone Enfants, Marseille, France : (2) UMR INSERM 910, Faculté de Médecine de la Timone, Marseille, France; (3) Chirurgie Orthopédique Infantile, Hôpital Lapeyronie, Montpellier, France; (4) Neurologie, Centre Hospitalier E.-Roux, Le Puy-en-Velay, France; (5) Pédiatrie, Centre Hospitalier, Annecy,
France: (7) Neurologie ENMG, Centre Hospitalier Lyon Sud, Pierre Bénite, France; (8) Médecine physique et réadaptation, Hôpital de l'Archet 1, Nice, France: (9) Institute of Clinical Neurophysiology, University Medical Centre Ljubljana, Slovenie ; (10) Clinique Neurologique, Hôpital R. Salengro, CHRU Lille, France ; (II) SEMMEN Unité d'Explorations Fonctionnelles Neurologiques, CH d'Agen, France ; (12) Laboratoire de Neurobiologie, Centre de Biologie et Pathologie Est, Hospices Civils de Lyon, Bron, France

Contact : rafaelle.bernard@ap-hm.fr

Charcot-Marie-Tooth disease (CMT) is a group of genetically and clinically heterogeneous hereditary motor and sensory neuropathies (HMSN). 40 genes and more than 60 loci have been identified to date. GDAP1 (Ganglioside Induced Differentiation-Associated Protein 1) belongs to the glutathione-S-transferase family and is probably involved in mitochondrial fission. Mutations in the GDAP1 gene have been clearly incriminated in recessive forms of both electrophysiological subtypes of CMT disease (demyelinating and axonal). The possible implication of GDAP1 in dominant forms of CMT has been suggested only twice (Claramunt R. et al., J Med Genet 2005 ; 42(4) : 358-65; Chung KW et al., J Hum Genet 2008 ; $53(4): 360-4)$, and GDAP1 is thus not routinely explored yet in a diagnostic setting in these cases. We report 11 new families in which a heterozygous mutation in GDAPI segregates with the disease, strongly suggesting a dominant mode of inheritance. We report 4 mutations, among whose, the Arg120Trp, that has already been described and is present in six families, while the three other mutations have never been reported. These are all missense variants and one of them, His123Arg, is present in three unrelated families. We discuss the pathogenicity of these variants in their clinical and genealogical context, and conclude that our data strongly suggest that GDAP1 should be routinely included in the molecular exploration of dominant forms of CMT.

Mots-clés : Charcot-Marie-Tooth disease, dominant inheritance, GDAP1.

P469. ASSOCIATION DE VARIANTS NON CLASSÉS DANS LES GENES MFN2 ET GDAPI CHEZ DEUX PATIENTS ATTEINTS D'UNE MALADIE DE CHARCOT-MARIE-TOOTH SÉVÈRE: HÉRÉDITÉ MENDÉLIENNE, EFFET MODULATEUR OU DIGÉNISME ?

N. Bonello (1, 2), C. Halbert (3), L. Viollet (4), B. Chabrol (3), N. Lévy $(1,2)$, R. Bernard $(1,2)$

(1) Département de Génétique Médicale, Hôpital Timone Enfants, Marseille, France; (2) UMR INSERM 910, Faculté de Médecine de la Timone, Marseille, France; (3) Service de Pédiatrie et Neurologie pédiatrique, Hôpital Timone Enfants, Marseille, France; (4) Service de Génétique Medicale, Hopital Necker Enfants Malades, Paris, France

Contact : rafaelle.bernard@ap-hm.fr

La maladie de Charcot-Marie-Tooth (CMT) est un groupe de neuropathies héréditaires sensitivo-motrices caractérisé par son hétérogénéité clinique et génétique. À ce jour 60 loci et 40 gènes sont identifiés. II s'agit d'une pathologie fréquente ( 1 cas sur 2500 ), pour laquelle la stratégie de diagnostic moléculaire a fait l'objet d'un consensus du réseau national des laboratoires en distinguant 6 cadres phénotypiques correspondant chacun à un schéma d'analyse « en cascade " de gènes à tester. Dans les formes sporadiques de CMT axonal (CMT2), l'analyse des gènes MFN2 (Mitofusin2) et GDAPI (Ganglioside Induced Differentiation-Associated Protein 1) est envisagée après exclusion des gènes GJB1 et P0. Nous rapportons ici deux cas de CMT2 sporadiques sévères associés à la présence conjointe d'une mutation hétérozygote dans GDAP1 et d'une autre dans MFN2. Le premier cas est celui d'une patiente chez laquelle les premiers signes ont été observés lors de l'acquisition de la marche (déformation des pieds en varus équin, et atteinte motrice des SPE). L'EMG montre une neuropathie sensitivomotrice axonale distale à forte prédominance motrice. À l'âge de 22 ans, la patiente présente une atteinte motrice nettement majorée au niveau distal, avec marche difficile. Chez cette patiente, nous avons identifié dans MFN2 le variant c. $311 \mathrm{G}>\mathrm{A} / \mathrm{p}$.Arg104Gln à l'état hétérozygote et hérité de sa mère asymptomatique, ainsi que le variant c.400delG/p.Asp134MetFSX13 dans GDAP1, hérité de son père qui présente une myotonie de Thomsen. Le second cas est celui d'un garçon chez lequel le diagnostic de CMT2 a été porté à l'âge de deux ans en raison de troubles de la marche. Ce patient actuellement âgé de 17 ans se déplace en fauteuil et a du subir une arthrodèse vertébrale en raison d'une scoliose invalidante. On notera de plus qu'il présente des difficultés d'apprentissage. Nous avons identifié chez lui le variant c. $310 \mathrm{C}>\mathrm{T} / \mathrm{p}$. Arg 104Trp dans MFN2 et le variant c.556A $>$ G/p.Ile186Val dans GDAP1. La mutation de MFN2 est une néomutation, tandis que la mutation de GDAP1 est héritée du père, par ailleurs totalement asymptomatique. En revanche, des signes très discrets de neuropathie ont été évoqués chez la mère et le grand-père 
maternel. Au total dans les deux cas nous discuterons la pathogénicité respective de ces variants de séquence non rapportés au préalable, mais aussi, associée, la question du mode de transmission de la maladie dans ces deux familles. Outre le fait que GDAP1, classiquement incriminé dans le CMT récessif, semble pouvoir être responsable de formes dominantes de CMT, et inversement pour MFN2, l'implication des protéines codées par ces deux gènes dans la dynamique mitochondriale (l'une pro-fusion, l'autre pro-fission) nous pousse à ne pas écarter la possibilité d'un effet conjoint, possiblement modificateur, de la présence simultanée de mutations dans ces deux gènes sur la physiopathologie de ces CMT.

Mots-clés : Charcot-Marie-Tooth, gènes modificateurs, digénisme.

\section{P470. ANOMALIES MOLECULAIRES DU GENE PMP22 DANS LES NEUROPATHIES DÉMYÉLINISANTES HÉRÉDITAIRES : HÉTÉROGÉNÉITÉ DU PHÉNOTYPE, DES TYPES DE MUTA- TIONS ET DU MODE D'HÉRÉDITÉ}

K. Larcher (1), S. Tardieu (1), A. Brice (1, 2), C. Verny (3), C. Cazeneuve (1), E. Leguern $(1,2)$

(1) Département de Génétique et Cytogénétique, Unité Fonctionnelle de Neurogénétique Moléculaire et Cellulaire, Groupe Hospitalier Pitié-Salpêtrière, AP-HP, Paris, France ; (2) Inserm UMR_S975, Groupe Hospitalier Pitié-Salpêtrière, Paris, France; (3) Département de Neurologie, Centre Hospitalier Universitaire, Angers, France

Contact : eric.leguern@psl.aphp.fr

Les réarrangements de la région $17 \mathrm{p} 11.2$ sont fréquemment impliqués dans le Charcot-Marie-Tooth de type 1 (CMT démyélinisant) et la neuropathie héréditaire avec hypersensibilité à la pression (NHHP) qui sont 2 neuropathies périphériques de transmission autosomique dominante. En effet, on constate que $70 \%$ des patients avec un CMT1 sont porteurs d'une duplication de $1,5 \mathrm{Mbp}$ de la région $17 \mathrm{p} 11.2$ et que $85 \%$ des sujets atteints de NHHP ont une délétion de la même région. Dans ces pathologies, le phénotype est du à l'effet de dose du gène PMP22 qui code pour une protéine de la myéline compacte du nerf périphérique. De rares mutations intragéniques de ce gène ont aussi été rapportées dans ces 2 phénotypes. Après avoir exclu les réarrangements de la région $17 \mathrm{p} 11.2 \mathrm{chez}$ les patients qui avaient été référés au laboratoire pour CMT1 ou NHHP, nous avons systématiquement séquencé les 4 exons codants du gène PMP22. Nous avons identifié des anomalies intragéniques chez 12 cas index, 7 sujets avec un diagnostic de CMT et 5 avec celui de NHHP. Parmi ces mutations, 7 conduisaient à un codon STOP prématuré et 5 à des mutations faux sens. Quatre des mutations faux sens étaient associées à un CMT et 1 à une NHHP. Il est intéressant de souligner qu'un des patients avec un CMT et consanguin était homozygote pour la nouvelle mutation c. $15 \mathrm{G}>\mathrm{C} / \mathrm{Leu} 5 \mathrm{Phe}$. Dans ce travail, nous avons confirmé que les anomalies intragéniques de PMP22 sont rares : $4 \%$ des 130 patients CMT et $11 \%$ des 36 patients NHHP sans réarrangement de la région $17 \mathrm{p} 11.2$ qui ont été suivis à la Pitié-Salpêtrière. De plus, les mutations faux sens peuvent mener à un gain de fonction probable car elles sont majoritairement associées au phénotype CMT mais aussi à une perte de fonction causant une NHHP. La situation est compliquée par l'identification de la première mutation faux sens à l'état homozygote qui provoque un CMT de grande sévérité. Nous discuterons des corrélations phénotype-génotype dans cette série.

Mots-clés : neuropathies, PMP22, diagnostic moléculaire.

IP471. NEUROPATHIES PÉRIPHÉRIQUES LIÉES À DES MUTATIONS DE LA MITOFUSINE 2 : SPECTRE CLINIQUE ET CORRÉLATIONS GÉNOTYPE-PHÉNOTYPE

B. Funalot (1-3), J. Calvo (1, 2, 15), R.A. Ouvrier (4), L. Lazaro (5), A. Toutain (6), P. De Mas (7), P. Bouche (8), B. Gilbert-Dussardier (9), M.C. Arne-Bes (10), J.P. Carrière (11) H. Journel (12), M.C. Minot-Myhie (13), C. Guillou (14), K. Ghorab (1, 2), L. Magy (1, 2), E. Sturtz, (3), J.M. Vallat $(1,2)$, C. Magdelaine (3)

(1) Centre de référence "neuropathies périphériques rares ", CHU de Limoges, Limoges, France ; (2) Service de neurologie, CHU de Limoges, Limoges, France ; (3) Service de biochimie et génétique moléculaire, CHU de Limoges, Limoges, France : (4) Institute for Neuromuscular Research, The Children's Hospital at Westmead, Westmead, Australia ; (5) Département de médecine de l'enfant et de l'adolescent, CHU de Rennes, Rennes, France; (6) Service de génétique, CHU de Tours, Tours, France; (7) Consultation de génétique, Clinique Saint-Jean-Languedoc, Toulouse, France ; (8) Département de neurophysiologie clinique, groupe hospitalier Pitié-Salpêtrière, Paris, France ; (9) Service de génétique médicale, CHU de Poitiers, Poitiers, France; (10) Service de neurologie et explorations fonctionnelles du systeme nerveux, CHU de Toulouse, Toulouse, France, (11) Service de neurologie pédiatrique, CHU de Toulouse, Toulouse. France; (12) Consultation de génétique médicale, $\mathrm{CH}$
Bretagne-Atlantique, Vannes, France; (13) Cabinet de neurologie, Rennes, France; (14) Service de rééducation fonctionnelle, CHU de Poitiers, Poitiers, France ; (15) Adresse actuelle : Instituto de Neurolgia, Hospital de Clinicas de la Facultad de Medicina, Universidad de la Repùblica, Montevideo, Uruguay.

Contact : benoit.funalot@unilim.fr

Introduction : Des mutations du gène de la mitofusine 2 (MFN2) ont été identifiées dans 10 à $20 \%$ des formes axonales de maladie de CharcotMarie-Tooth (CMT2A2), de transmission autosomique dominante et de sévérité variable. Objectifs: Identifier des mutations de MFN2 dans un échantillon de patients atteints de neuropathies périphériques, décrire le phénotype associé et établir des corrélations génotype-phénotype. Méthodes: 150 cas index atteints de neuropathies périphériques génétiquement déterminées (ou présumées telles pour les cas sporadiques) ont été explorés par séquençage du gène MFN2 dans notre laboratoire. Des mutations des gènes GJB1 (connexine 32) et MPZ (P0) avaient été exclues au préalable. Les données cliniques et paracliniques ont été recueillies sur un formulaire standardisé. La sévérité de la neuropathie a été évaluée par l'utilisation du « Charcot-Marie-Tooth disease neuropathy score ». Résultats : 107 patients étaient atteints de formes axonales (CMT2 : Vitesse de conduction nerveuse (VCN) du nerf médian $>38 \mathrm{~m} / \mathrm{s}$ ), 43 patients de formes « intermédiaires " $(\mathrm{VCN}>25 \mathrm{~m} / \mathrm{s})$. Des mutations ont été identifiées dans 19 cas index de CMT2 $(17,8 \%)$ et chez un patient avec forme intermédiaire $(\mathrm{VCN}=35 \mathrm{~m} / \mathrm{s})$. Le mode de transmission de la neuropathie était autosomique dominant dans 8 cas, autosomique récessif dans 3 cas et apparemment sporadique dans 9 cas (dans 4 cas sur 9 , la survenue d'une néomutation chez le cas index a pu être démontrée). Un total de 25 patients appartenant à ces 20 familles a été examiné. Le phénotype allait de formes légères à des formes sévères à début précoce. Des signes associés ont été observés chez 8 patients ( $32 \%$ ). Six patients ont subi une biopsie de nerf sural et dans tous les cas l'examen de coupes longitudinales du nerf en microscopie électronique montrait des anomalies marquées des mitochondries axonales (petites mitochondries arrondies et agrégées à la périphérie de l'axone), Discussion: Le phénotype clinique des patients avec mutations MFN2 apparaît similaire à celui de précédentes séries : la sévérité de la neuropathie est variable, et certains patients présentent des signes associés : syndrome pyramidal réflexe, asymétrie de la neuropathie, surdité, atrophie optique,... Dans 6 des 7 cas sévères, les mutations responsables étaient localisées dans ou juste en amont du domaine GTPase de la mitofusine 2. Conclusion: Les mutations de MFN2 sont une cause fréquente de CMT2, dont la sévérité et le mode de transmission sont variables. La recherche de mutations de MFN2 doit être un examen de première ligne chez tous les patients atteints de CMT2, quel que soit la gravité ou le mode de transmission de la neuropathie (dominante, récessive ou sporadique).

Mots-clés : Charcot-Marie-Tooth (maladie de), mitofusine.

\section{IDENTIFICATION OF NOVEL GDAP1 MUTATIONS CAU- SING AUTOSOMAL RECESSIVE CHARCOT-MARIE-TOOTH DISEASE IN 3 FAMILIES}

M.A.M. Salih (1), H.E. Mikesova (2), S.A. Elmalik (3), M.M. Kabiraj (4), A.E.M. Ahmed (5), M.M. Mukhtar (6), E. LeGuern (2, 7), H. Azzedine (2) (1) Division of Pediatric Neurology, Department Of Pediatrics, College of Medicine, King Saud University, Riyadh, Saudi Arabia ; (2) CRICMINSERM-U975, Hôpital de la Pitié-Salpêtrière, Paris, France ; (3)Department of Physiology, College of Medicine, King Saud University, Riyadh, Saudi Arabia ; (4) Division of Clinical Neurophysiology, Department of Neuroscience, Armed Forces Hospital, Riyadh, Saudi Arabia ; (5)Department of Physiology, Faculty of Medicine, University of Khartoum, Sudan; (6) Institute of Endemic Diseases, Faculty of Medicine, University of Khartoum, Sudan; (7) UF de génétique, Département de Génétique et Cytogénétique, Hôpital de la Pitié-Salpêtrière, Paris, France

Contact : azzedine.hamid@yahoo.fr

We describe 3 consanguinous families with autosomal recessive axonal phenotype of Charcot-Marie- Tooth disease (ARCMT2). These consisted of 16 individuals aged 2-35 years, 5 of them were affected. Onset was about 2 years with delayed walking, evolving into high steppage gait and distal lower limb muscle weakness and wasting. Cognitive development was normal, ambulation was preserved and no scoliosis was detected in the affected individuals. Nerve conduction studies were compatible with axonal CMT. However, nerve pathology showed prominent loss of large myelinated axons and typical onion bulbs formations. These families were screened for 12 microsatellites markers covering the 2 more frequent ARCMT2 loci (CMT4A/2K and CMT2B1). Assignment of the families to the CMT4B/2K was established by homozygocity mapping and confirmed by linkage analysis. Sequencing of GDAP1 was performed in all members of the 3 families and 3 new mutations were identified. To the best of our 
knowledge, these are the first GDAP1 mutations reported in the Arabian Peninsula. The phenotype is of axonal type and is compatible with the majority of previously reported cases.

Mots-clés : CMT, GDAP1.

\section{P473. ÉTUDE DES MANIFESTATIONS NEUROLOGIQUES ET DES ASPECTS IRM DANS LE SYNDROME DE WOLFRAM SUR UNE COHORTE DE 59 PATIENTS}

A. Chaussenot (1), S. Bannwarth (1, 2), B. Vialettes (3), B. Chafino (1), S. Mutz (1), B. Chabrol (4), Groupe Français du Syndrome de Wolfram, V. Paquis-Flucklinger $(1,2)$

(1) Service de génétique médicale, Hôpital Archet 2, CHU de Nice, France: (2) IGMRC, FRE CNRS/UNSA 3086, Université de Nice Sophia-Antipolis, France ; (3) Service de Nutrition, Hôpital Timone, CHU de Marseille, France; (4) Service de Neuropédiatrie, Hôpital Timone, $\mathrm{CHU}$ de Marseille, France

Contact : chaussenot.a@chu-nice.fr

Le syndrome de Wolfram (WS) est une maladie rare, de transmission autosomique récessive, caractérisée par la survenue précoce d'un diabète insulino-dépendant (DID) et d'une atrophie optique (AO), fréquemment associés à un diabète insipide, une surdité et des troubles neurologiques. Cette pathologie est hétérogène sur le plan génétique : le gène WFS1 est majoritairement impliqué dans les populations caucasiennes alors que WFS2 a été associé au WS dans des familles jordaniennes. L'objectif de notre étude était d'analyser les caractéristiques neurologiques et IRM observées dans cette pathologie, peu décrites à ce jour, et de rechercher d'éventuelles corrélations génotype/phénotype. Nous avons étudié de manière rétrospective les 59 patients pour lesquels une analyse du gène WFS1 a été réalisée dans le laboratoire de génétique du $\mathrm{CHU}$ de Nice et qui présentaient 2 mutations identifićes et/ou l'association d'un DID et d'une AO avant l'âge de 30 ans. Au sein de notre cohorte constituée de 32 hommes et 27 femmes d'âge moyen 26 ans (5-57), $97 \%$ avaient développé un DID à un âge moyen de 9 ans ( 8 mois-38) et $92 \%$ une AO à un âge moyen de 14 ans (3-43). Les manifestations neurologiques, 3ème atteinte en terme de fréquence $(47 \%)$ après le DID et l'AO, débutaient à un âge moyen de 20 ans (4-47), âge de 10 ans plus précoce que celui rapporté dans la littérature sur des séries inférieures en nombre. Par ordre de fréquence décroissant, les signes neurologiques retrouvés étaient : un syndrome cérébelleux $(54 \%)$, des troubles cognitifs $(25 \%)$, une épilepsie $(21 \%)$, une neuropathie périphérique $(21 \%)$, un nystagmus $(21 \%)$, des troubles de la déglutition $(14 \%)$, une dysautonomie $(10 \%)$ et une atteinte des paires crâniennes $(7 \%)$. Il faut noter que les troubles cognitifs retrouvés étaient plus fréquents dans notre série que ceux rapportés précédemment dans la littérature. Concernant les aspects IRM, l'atrophie marquée du cervelet et du tronc cérébral était prépondérante $(80 \%)$ et corroborait les données cliniques et celles de la littérature. En revanche, nous rapportons pour la première fois dans cette pathologie 2 cas avec une atteinte diffuse et symétrique de la substance blanche péri-ventriculaire. Sur le plan moléculaire, nous avons identifié 2 mutations du gène WFS1 chez 53 patients, 1 seule mutation a été retrouvée chez 3 patients et aucune chez les 3 derniers. Nous confirmons nos résultats précédents qui montraient que la présence de mutations faux sens est corrélée de manière significative avec un âge de début plus tardif du DID et de l'AO. Par contre, le nombre de mutations faux-sens et de mutations inactivatrices est identique chez les patients avec et sans signe neurologique. Cette étude, qui concerne le nombre de patients le plus important rapporté à ce jour, permet de mieux définir l'atteinte neurologique qui conditionne le pronostic vital dans ce syndrome.

Mots-clés : syndrome de wolfram, WFS1, signes neurologiques.

\section{P474. PERTE DE FONCTION DE LA PROTÉINE CASK PAR DUPLICATION PARTIELLE DU GENE}

C. Bonnet (1, 2), C. Bénéteau (3), M. Béri (1, 2), B. Léotard (1), A. Perrin

(1), B. Leheup $(2,3)$, P. Jonveaux $(1,2)$

(1) Laboratoire de Génétique, CHU Nancy, Vandouvre les Nancy, France; (2) Nancy Université - EA 4368, Laboratoire de Génétique, Centre Hospitalier Universitaire de Nancy, Vandauvre les Nancy, France : (3) Service de Médecine Infantile III et Génétique Clinique, CHU Nancy, Vandouvre les Nancy, France

Contact : ce.bonnet@chu-nancy.fr

Nous avons identifié par CGH-array (Agilent $244 \mathrm{~K}$ ) une duplication partielle de novo du gène CASK en Xp11.4 chez une fille âgée de 4 ans présentant un tableau d'encéphalopathie avec microcéphalie, hypoplasie du cervelet et du tronc cérébral et un syndrome de West. La duplication a une taille minimale de $143 \mathrm{~kb}$ et touche les exons 6 à 25 du gène CASK. réalisées sur l'ARN de la patiente ont montré d'une part que le niveau d'expression du gène est normal et d'autre part que la duplication des exons 6 à 25 est présente au niveau de l'ARN. Le séquençage du point de jonction confirme la duplication en tandem qui n'entraîne pas de décalage du cadre de lecture. La duplication partielle du gène est donc responsable de la synthèse d'un transcrit aberrant porteur d'une duplication multiexonique. La protéine CASK normale à une taille de 926 acides aminés et la duplication serait à l'origine de la synthèse d'une protéine de 1623 acides aminés. La duplication partielle du gène CASK serait donc responsable d'une perte de fonction de la protéine soit par dégradation post-traductionnelle de la protéine anormale soit par modification de la structure tridimensionnelle de la protéine si elle n'est pas dégradée. Le gène CASK code une protéine kinase qui joue un rôle important dans le développement et les fonctions cérébrales. CASK interagit dans le noyau des neurones avec un facteur de transcription (TBR1 : T-brain-1) dont NR2b (NMDA receptor 2b) est une des cibles. Des mutations ponctuelles, un inversion interrompant le gène et des délétions toutes responsables d'une perte de fonction de CASK ont été décrites chez des filles sans biais d'inactivation de l'X et présentant le même tableau clinique que notre patiente: retard mental sévère, microcéphalie et hypoplasie du tronc cérébral et du cervelet. La perte de fonction ne serait pas viable chez les garçons hémizygotes. Nous décrivons donc chez une fille présentant retard mental, microcéphalie et hypoplasie du tronc cérébral et du cervelet la première duplication partielle du gène CASK à l'origine d'une perte de fonction protéique.

Mots-clés : retard mental, CASK, perte de fonction.

\section{P475. APPORT DU SCREENING DES GENES PEX DANS LES DÉFAUTS DE BIOGENĖSE DES PEROXYSOMES}

E. Luangkhot (1), B. Doray (2), F. Couderc (3), A. Chamouine (4), D. Amsalem (4), S. Lyonnet (5), A. Pennerath (6), H. Ogier (7), B. Chabrol (8), C. Tranchant (9), T. Levade (10), E. Bieth (11), P. Calvas (11), N. Guffon (12), C. Vianey-Saban (1), D. Cheillan (1)

(1) Service maladies héréditaires du métabolisme et dépistage néonatal, Hospices Civils de Lyon, France; (2) Service de génétique médicale, Hôpital Hautepierre, Strasbourg, France; (3) Service de pédiatrie, CH d'Aix-en-Provence, France ; (4) Réanimation infantile et service de pédiatrie, CHU de Besançon, France; (5) Service de génétique médicale, Hôpital Necker Enfants Malades, Paris, France; (6) Service de pédiatrie, $\mathrm{CH}$ de Colmar ; (7) Centre de référence maladies héréditaires du métabolisme, Hôpital Robert Debré, Paris, France; (8) Centre de référence maladies héréditaires du métabolisme, Hôpital de la Timone, Marseille, France: (9) Département de neurologie, Hôpital Hautepierre, Strasbourg, France: (10) Laboratoire de biochimie, Hôpital Purpan, Toulouse, France; (11) Service de génétique médicale, Hôpital Purpan, Toulouse, France: (12) Centre de référence maladies héréditaires rénales et du métabolisme, Hospices Civils de Lyon, France

Contact : david.cheillan@chu-lyon.fr

Les défauts de biogenèse des peroxysomes (DBP) sont des maladies rares à transmission autosomique récessive dont la fréquence est d'environ 1/50 000 dans la population générale. Trois entités cliniques peuvent être distinguées : le syndrome de Zellweger (ZS), l'adrénoleucodystrophie néonatale (NALD) et la maladie de Refsum infantile (IRD). Ces affections sont causées par le déficit d'une des protéines de la membrane peroxysomale appelées peroxines, chacune d'entre elles étant codée par un gène PEX différent (13 gènes PEX sont impliqués en pathologie humaine). Leur diagnostic repose à la fois sur des arguments cliniques et biologiques. Néanmoins, le diagnostic de certitude est apporté par la détection de mutations dans un des gènes PEX après des étapes de biologie cellulaires longues et complexes d'interprétation (études de complémentation sur culture de fibroblastes). L'objectif de ce travail était de développer un protocole de screening des gènes PEX reposant sur la recherche des mutations les plus fréquentes sans étude de complémentation préalable. Seize exons répartis sur 6 gènes PEX différents (PEX1, PEX2, PEX6, PEX10, PEX12 et PEX26) ont été sélectionnés couvrant plus de $80 \%$ des allèles mutés. Ce protocole a été appliqué chez 15 patients atteints d'un DBP caractérisé au plan biochimique. Grâce à notre approche, nous avons mis en évidence 2 allèles pathogènes chez 6 patients, 2 d'entre eux possédant un troisième allèle pathogène sur un autre gène PEX, dont la pathogénicité reste à démontrer. Un seul allèle pathogène a été identifié chez 3 patients. En revanche, pour les 6 autres patients, le screening s'est révélé négatif. Parmi les 8 mutations différentes détectées, 3 n'ont jamais été décrites mais sont probablement pathogènes car elles affectent un domaine très conservé de chacune des protéines: p.Cys796Arg (PEX6 - Exon 13, c.2392T >C), p.Cys296Phe (PEX10 - Exon 5, c.887G > T) et p.Trp250Arg (PEX2 - Exon 4, c. $748 \mathrm{~T}>\mathrm{C}$ ). Notre protocole a permis de déterminer, de façon simple et rapide, le gène PEX en cause chez $60 \%$ des patients, confirmant ainsi le 
diagnostic de DBP et ouvrant la possibilité à une étude familiale et au diagnostic prénatal souvent difficile avec les méthodes biochimiques. À partir de ces résultats, nous proposons une stratégie d'exploration des patients suspects de DBP, reposant à la fois sur les données cliniques, biochimiques et enzymatiques ainsi que sur le screening des gènes PEX.

Mots-clés : peroxysome, gene PEX, screening.

E476. IMPLICATION DU GENE MFN2 DANS LES ATROPHIES OPTIQUES AUTOSOMIQUES DOMINANTES PLUS

P. Ribaï (1), G. Picard (2), F. Liénard (2), P. Jacquerye (2), N. Van Regemorter (3), N. Lannoy (4), P. Hilbert (1), C. Verellen-Dumoulin (1)

(1) Institut de Pathologie et de génétique, Gosselies, Belgique; (2) Clinique St-Pierre, Ottignies, Belgique; (3) Hôpital Erasme, Bruxelles, Belgique ; (4) Cliniques St-Luc, Bruxelles, Belgique

Contact : pascale.ribai@ipg.be

L'atrophie optique autosomique dominante (AOAD) se manifeste cliniquement par une baisse insidieuse de l'acuité visuelle dans les deux premières décennies, une dyschromatopsie et une pâleur rétinienne centrale au fond d'œil. Cependant, la découverte du gène OPA 1 , majoritairement impliqué dans les $\mathrm{AOAD}$, a permis la description de patients présentant un phénotype plus complexe appelé AOAD-plus. Celui-ci, en plus de l'atrophie optique, se caractérise par la présence de signes divers tels que surdité, ataxie cérébelleuse, ophtalmoplégie externe progressive ou tableau clinique évoquant une sclérose en plaques. Ces signes additionnels dans l'AOAD-plus ont été liés à la présence de délétions multiples dans l'ADN mitochondrial et une baisse de l'activité de la cytochrome c oxydase, observés dans les muscles et les fibroblastes de patients. Ces éléments ont permis de mieux comprendre la fonction de la protéine opal, impliquée dans le maintien du réseau mitochondrial, la phosphorylation oxydative et l'apoptose. Nous décrivons le tableau clinique et génétique de 8 patients appartenant à une famille, qui présentent une $\mathrm{AOAD}$ liée à la mutation c. $448+1 \mathrm{G}>\mathrm{C}$ dans l'intron 3 du gène OPA1. En plus de l'atrophie optique, trois de ces patients présentent des anomalies de la substance blanche cérébrale, une neuropathie axonale sensitivo-motrice, une paraplégie spastique ou des signes pyramidaux à l'examen. En raison de l'association atrophie optique et neuropathie périphérique, nous avons analysé le gène MFN2. En effet, les mutations de ce gène entrainent une neuropathie sensitivomotrice axonale dans le cadre de la maladie de Charcot-Marie-Tooth de type $2 \mathrm{~A} 2$. Un variant allélique de ce gène est responsable de l'association neuropathie périphérique, atrophie optique et/ou anomalies de la substance blanche cérébrale. La mutation c.892G $>$ A dans l'exon 9 du gène MFN2 a été trouvée chez les 3 patients présentant un phénotype d'AOAD-plus. Celle-ci n'a jamais été décrite chez un autre patient mais concerne un nucléotide conservé entre plusieurs espèces et n'est pas rapportée comme polymorphisme. Cette mutation ségrège dans notre famille avec le phénotype AOAD-plus. Les protéines opal et $\mathrm{mfn} 2$ sont toutes deux impliquées dans la fusion des mitochondries et agissent comme partenaires pour le maintien du réseau mitochondrial. Le dysfonctionnement concomitant de ces deux protéines pourrait entraîner un phénotype plus sévère et complexe que celui résultant de l'altération de chacune d'elles. Dans notre famille, les mutations dans les gènes OPA1 et MFN2 pourraient expliquer la présence d'une neuropathie périphérique et les anomalies de la substance blanche cérébrale en plus de l'atrophie optique. Nous formulons l'hypothèse que le phénotype complexe décrit dans les AOAD-plus pourrait résulter de mutations concomitantes dans le gène OPA1 et un autre gène, dans notre cas le gène MFN2.

Mots-clés : atrophie optique, OPA1, MFN2.

\section{P477. QUATRE NOUVEAUX CAS DE DYSTROPHIES COR- NÉENNES LIÉES À DES MUTATIONS PARTICULIÈRES DU GENE TGFBI}

F. Niel-Butschi (1), M. Boimard (2), J. Iwaszkiewicz (3), B. Kantelip (4), F. D'hermies (5), B. Dubosq (6), M. Montard (6), C. Hamel (7), G. Renard (8), J.-L. Bourges (8), P.-J. Pisella (9), V. Zoete (3), M. Delpech (2), S. Valleix (2)

(1) Service de Génétique Médicale, Centre Hospitalier Universitaire Vaudois, Lausanne, Suisse; (2) Laboratoire de Biochimie et Génétique Moléculaire, Hôpital Cochin-Pavillon Cassini, Paris, France; (3) Molecular Modeling Group, Institut Suisse de Bioinformatique, Lausanne, Suisse ; (4) Laboratoire d'anatomopathologie, CHU de Besançon, France ; (5) Laboratoire d'anatomopathologie, Hôpital de l'Hôtel-Dieu, Paris, France ; (6) Service d'Ophtalmologie, $\mathrm{CHU}$ de Besançon, France; (7) Centre National de maladies rares- Affections sensorielles génétiques. CHRU de Montpellier, France: (8) Service d'Ophtalmologie, Hôpital de l'Hôtel-Dieu, Paris, France: (9) Service d'Ophtalmologie, CHU de Tours, France

Contact : sophie.valleix@cch.aphp.fr
Les dystrophies héréditaires de la cornée liées au gène TGFBI sont des affections transmises sur un mode autosomique dominant qui se caractérisent par la présence de dépôts au sein du stroma cornéen, altérant progressivement la transparence de la cornée. Le gène TGFBI code la kératoépithéline, protéine ubiquitaire impliquée dans des processus de croissance et différenciation cellulaires ainsi que dans la réparation tissulaire. Le plus remarquable dans ces pathologies est l'atteinte isolée de la cornée et la variabilité de la nature des dépôts selon le type de mutations faux-sens de la kératoépithéline. C'est à notre connaissance le seul modèle héréditaire actuellement connu où une même protéine selon le type de mutations puisse être impliquée dans la formation de dépôts amyloïdes, granulaires, mixtes ou encore fibrocellulaires. C'est pourquoi ces dystrophies cornéennes sont un modèle unique de « pathologies conformationnelles » dont l'étude contribuerait à élucider les mécanismes amylö̈des en général. Nous avions précédemment rapporté le spectre des mutations du gène TGFBI qui nous avait permis d'établir une corrélation génotypephénotype à partir de notre population française, et nous avions décrit de nouvelles mutations associées à de nouvelles présentations cliniques. Dans cette étude, nous présentons le phénotype de quatre nouveaux cas de dystrophies liées à des mutations nouvelles, ou rapportées qu'une seule fois, du gène TGFBI. Ces mutations faux-sens ont été identifiées dans le $4^{\text {c }}$ domaine FAS1 de la kératoépithéline, homologue au domaine FAS1 de la fasciculine 1 dont la structure NMR a récemment été déterminée. Ainsi grâce à cette homologie des domaines FAS1, nous rapportons à l'aide de différents logiciels de modélisation l'impact de ces nouvelles mutations identifiées. Trois des mutations apparaissent être critiques pour la stabilité structurale de la kératoépithéline et peuvent ainsi expliquer le phénotype amyloïde de ces dystrophies, alors que la dernière mutation pourrait altérer des interactions de la kératoépithéline avec des protéines de la matrice extracellulaire expliquant que les dépôts caractérisés dans cette dystrophie soient de nature non amyloïde.

Mots-clés : TGFBI, dystrophies cornéennes héréditaires, modélisation.

IP478. SPECTRE DES MUTATIONS DU GENNE ABCA4 DANS UNE GRANDE COHORTE DE PATIENTS FRANCAIS ATTEINTS DE DYSTROPHIE MACULAIRE AUTOSOMIQUE RÉCESSIVE

C.M. Dhaenens (1), J. Leclerc (1), C. Piriou (1), A. Devos (1), S. Defoort (2), C. Hamel (3), B. Puech (2), V. Dumur (1), D. Allorge (1)

(1) UF Génopathies, Laboratoire de Biochimie et Biologie Moléculaire, Centre de Biologie et Pathologie, CHRU de Lille. France; (2) Service d'Explorations Fonctionnelles de la Vision, Hôpital Salengro, Lille, France; (3) Centre National de Référence des maladies rares, Hôpital Gui de Chauliac, Montpellier, France

Contact : c-dhaenens@chru-lille.fr

Le gène $\mathrm{ABCA} 4(\mathrm{ABCR})$ est impliqué dans plusieurs formes de dystrophies maculaires héréditaires, dont la maladie de Stargardt (STGD1), qui est la forme la plus fréquente, la dystrophie des cônes et des bâtonnets (CRD3), la rétinite pigmentaire (RP19), et le Stargardt de l'adulte (ARMD). Cette étude rapporte les données obtenues par criblage moléculaire du gène ABCA4 réalisé chez 307 familles françaises présentant une dystrophie maculaire autosomique récessive. La majorité d'entre elles $(66 \%)$ présentait un tableau clinique classique de maladie de Stargardt. L'analyse des 50 exons a été réalisée par dHPLC (chromatographie liquide haute performance en condition dénaturante) et/ou par séquençage. Sur l'ensemble de notre cohorte, nous avons pu mettre en évidence les deux mutations responsables chez $49 \%$ des patients et une seule mutation chez $27 \%$ d'entre eux. Dans $24 \%$ des cas, aucune mutation n'a été identifiée. Notre technique d'analyse nous permet ainsi de confirmer le diagnostic clinique et/ou l'implication de $\mathrm{ABCA} 4$ chez près de $76 \%$ des patients présentant une dystrophie maculaire. Le taux de détection atteint $89 \%$ dans les formes typiques de Stargardt. Nous retrouvons ainsi deux mutations dans $60,3 \%$ des cas de STGD1, et une seule mutation dans $28,3 \%$ des cas. À l'inverse, $68 \%$ des formes atypiques ne sont pas liées à $\mathrm{ABCA} 4$. L'implication de ce gène est également importante dans les dystrophies cônes-bâtonnets, puisque $35 \%$ de ces patients portent deux mutations et $22 \%$ une mutation de ABCA4. L'absence de second évènement mutationnel chez certains patients peut être liée à la présence de délétions partielles ou totales d'un allèle, non recherchées lors de ce criblage, ou encore à la présence de mutations situées en dehors des régions analysées par nos techniques. Au total, le criblage nous a permis de mettre en évidence 186 mutations dont 97 n'ayant pas été répertoriées précédemment dans la littérature. Les variants sont principalement des mutations faux-sens $(63,9 \%)$, des petites délétions ou insertions $(12,3 \%)$ et des mutations affectant les sites invariants d'épissage $(12,9 \%)$. Elles sont réparties de façon homogène le long du gène, ne permettant pas de définir la présence de $«$ points chauds $»$ mutationnels. Il s'agit principalement de mutations privées : $65 \%$ des mutations n'ont en effet été retrouvées que chez un patient. 
Seuls quelques variants ont été identifiés à plusieurs reprises. Les mutations les plus fréquentes dans notre population sont: p.Gly1591Arg $(6,7 \%)$, p.Gly196IGlu (6\%), p.Gly863Ala (4,7\%) et p.Ala1038Val (3\%). A notre connaissance, aucune étude n'avait précédemment porté sur une cohorte aussi importante. Nous avons ainsi pu évaluer l'implication de ABCA4 dans différentes formes de dystrophies maculaires, ainsi que la fréquence et la grande diversité des mutations observées dans la population française.

Mots-clés : ABCA4, STARGARDT, mutations.

DP479. MICROSPHÉROPHAKIE ISOLÉE ET SYNDROME DE WEILL MARCHESANI: MALADIES ALLÉLIQUES LIÉES À ADAMTS10 ?

F. Ouechtati (1, 2), S. Ben Yahia (3), B. Jelliti (3), S. Nouira (1), S. Chakroun (1), M. Khairallah (3), S. Abdelhak (1)

(I) UR04SP03 Etude moléculaire des maladies orphelines d'origine génétique, Institut Pasteur de Tunis, Tunisie; (2) URI7/04 Oculogénétique, Institut Hédi Rais d'Ophtalmologie de Tunis, Tunisie ; (3) 02/UR/08-17 Eil et maladies systémiques, Faculté de Médecine de Monastir, Tunisie Contact : farah.ouechtati@gmail.com

La microsphérophakie est une atteinte rare du pôle antérieur caractérisée par une microphakie et une sphérophakie bilatérale, un cristallin petit et sphérique, et une forte myopie, due à un défaut d'accomodation. Cette pathologie a essentiellement été rapportée en association avec une ectopie du cristallin, un glaucome, et souvent avec des atteintes systémiques comme le Weill Marchesani et le syndrome marfanoïde. Une anomalie du corps ciliaire et des zonules semble être la cause de cette atteinte cristallinienne. Cependant aucune étude n'a permis de définir la nature moléculaire. Nous rapportons le neuvième cas familial de microsphérophakie isolée depuis 1901. La famille est consanguine et comporte deux cas atteints, une fille et un garçon, ne présentant aucune atteinte extraoculaire ou systémique. Une étude de liaison ciblant le locus arWMS a été effectuée au moyen de 4 STRs. Selon la stratégie d'homozygotie par descendance, nous avons pu établir une exclusion du gène ADAMTS10. D'autres gènes candidats COL5A3; ICAM1, $-4-5$; SMARCA4, TUBB4, ARHGEF18, BEST2, C3, RDH8, OLFM2, CRB3, CACNA1A, PDE4A et FBN3 ont également été exclus. Notre étude démontre que la microsphérophakie isolée et le syndrome du Weill Marchesani ne sont probablement pas alléliques au gène ADAMTS10.

Mots-clés : ADAMTS10, homozygotie par descendance, exclusion.

\section{IP480. CONFIRMATION DE L'IMPLICATION DU GÉNE ADAMTSLA DANS L'ECTOPIE CRISTALLINIENNE AUTOSO. MIQUE RÉCESSIVE}

C. Stoetzel (1), V. Bennouna Greene (1), V. Pelletier (2), Y. PerdomoTrujillo (2), L. Liebermann (2), V. Marion (1), H. De Korvin (3), C. Boileau (4), J.L. Dufier (5), H. Dollfus $(1,2)$

(I) Laboratoire de Génétique Médicale EA3949, Équipe Avenir-Inserm, Faculté de Médecine de Strasbourg, Université de Strasbourg, France: (2) Centre de Référence pour les Affections Rares en Génétique Médicale (CARGO) et Service de Génétique Médicale, Hôpitaux Universitaires de Strasbourg, France; (3) Service d'Ophtalmologie, Hôpitaux Civils de Colmar, France; (4) Laboratoire de Biochimie, d'Hormonologie et de Génétique Moléculaire, Hôpital Ambroise Paré, Hôpitaux de Paris, France ; (5) Service d'Ophtalmologie, Hôpital Necker - Enfants Malades Assistance Publique - Hôpitaux de Paris, France

Contact : corinne.stoetzel@medecine.u-strasbg.fr

L'ectopie cristallinienne est une maladie des fibres de la zonula, appareil fibrillaire qui suspend le cristallin dans l'aire pupillaire. L'altération des fibres zonulaires se manifeste par une luxation partielle ou complète du cristallin par rapport à sa position physiologique compromettant progressivement la fonction visuelle jusqu'à ce qu'une chirurgie soit pratiquée. L'ectopie cristallinienne est le plus souvent associée à des maladies systémiques telles que le syndrome de Marfan, le syndrome de Weill-Marchesani ou l'homocystinurie. L'ectopie cristallinienne isolée non syndromique, est plus rare et de transmission autosomique les formes dominantes plus fréquentes que les formes récessives. Récemment, des mutations dans le gène LTBP2 ont été décrites (Ali $\mathrm{M}$ et al Am J Hum Genet. 2009 May) dans des cas d'ectopie cristallinienne autosomique récessive associées ou non à d'autres manifestations oculaires (ex : glaucome) ou extra oculaire (ex : habitus marfanoïde). Très récemment, il a été démontré que le gène ADAMTSL4 est impliqué dans la forme autosomique récessive d'ectopie cristallinienne isolée pure dans une famille consanguine (Ahram D et al., Am J Hum Genet. 2009 Feb). Nous présentons une famille consanguine avec deux enfants atteints d'une forme homozygote IVS4-1G>A/IVS4-1G >A dans le gène ADAMTSLA. Cette observation (deuxième rapportée dans la littérature) confirme bien la participation de ce gène dans cette pathologie et souligne le rôle principal des protéases d'ADAMTS dans l'homéostasie des fibres de la zonula.

Mots-clés : ectopie cristallinienne, ADAMTSL4.

IP481. SPECTRE DES MUTATIONS DU GENE SPATA7 DANS UNE COHORTE DE PATIENTS ATTEINTS D'AMAUROSE CONGÉNITALE DE LEBER ET ÉTABLISSEMENT DE PREMIÈRES CORRÉLATIONS GÉNOTYPE-PHÉNOTYPE

I. Perrault (1), N. Delphin (1), X. Gerard (2), S. Gerber (1), S. Hanein (1), V. Pelletier (3), H. Dollfus (3), B. Puech (4), S. Defoort-Dhellemes (4), A. Munnich (1), O. Roche (5), J. Kaplan (1), J.M. Rozet (1)

(1) INSERM U781 - Université Paris-Descartes, CHU Necker, Paris, France : (2) Genethon Évry, France ; (3) Service de Génétique Médicale, Hôpitaux Universitaires de Strasbourg, France; (4) Service d'Ophtalmogie, CHRU Roger-Salengro, Lille, France; (5) Service d'Ophtalmogie - Université Paris-Descartes, CHU Necker, Paris, France

Contact : isabelle.perrault@inserm.fr

L'amaurose congénitale de Leber (ACL) est la plus précoce et la plus sévère des dystrophies rétiniennes. Deux-sous types ont été individualisés. Le type I désigne une cone-rod dystrophy congénitale et stationnaire, dramatiquement sévère. Le type II désigne une rod-cone dystrophy certes sévère mais progressive, représentant l'extrémité d'un spectre de gravité des rétinopathies pigmentaires (RP). À ce jour, quatorze gènes d'ACL ont été identifiés. Jusqu'en 2008, l'analyse clinique des patients porteurs de mutations dans les 13 premiers gènes avait permis de statuer que $3 / 13$ rendaient compte du type I $(38,1 \%$ de l'ensemble des cas) et $10 / 13$ du type II ( $25,6 \%$ de l'ensemble des cas). La quasi-totalité des protéines codées par ces gènes sont très préférentiellement voire spécifiquement exprimées dans la rétine et sont responsables d'ACL strictement isolée. Par contraste, LCA5 et CEP290 sont largement exprimés et les mutations de ce dernier gène ont été reconnues responsables de phénotypes variés allant d'ACL type $\mathrm{I}$ isolée à des troubles pléiotropiques incluant certains sous-types de syndrome de Joubert (JBTS5) ou certains sous-type de syndrome Senior-Loken (SNLS6). Le dernier des gènes identifiés, SPATA7, code également une protéine exprimée au-delà de la rétine et est incriminé dans la survenue d'ACL ou de RP précoces récessives autosomiques. Les travaux que nous présentons avaient pour objectif d'évaluer l'implication de ce nouveau gène dans une cohorte de 139 patients non apparentés et non porteurs de mutations dans les autres gènes, atteints d'ACL isolée $(n=134 / 139)$ ou syndromique $(2 / 139$ SNLS, $2 / 139$ JBTS et $1 / 139$ Alstrom). Six mutations différentes ont été identifiées dans quatre familles d'ACL isolée ( $1 / 6$ homozygote) ; 4/6 de ces mutations sont originales. Bien que le gène SPATA7 soit exprimé au-delà de la rétine, aucune mutation n'a été identifiée dans les 5 familles d'ACL syndromique. Cette recherche devra être réalisée dans un échantillon très élargie pour être concluante. L'analyse clinique fine des patients des 4 familles permet d'inclure le gène SPATA7 comme onzième gène des ACL de type II avec une fréquence rare ( $1,1 \%$ de l'ensemble des cas). Ce résultat conforte l'idée que les mutations qui restent à découvrir, à l'origine de la maladie chez environ $35 \%$ des patients, concerneront vraisemblablement des gènes impliqués chacun dans un petit nombre de familles, rendant malaisée leur identification.

Mots-clés : Amaurose congénitale de Leber, gène SPATA7, corrélations génotype-phénotype.

E482. STRATÉGIE D'ANALYSE MOLÉCULAIRE ET PREMIERS RÉSULTATS POUR LE DIAGNOSTIC ET L'IDENTIFICATION DE NOUVEAUX GENES/LOCI DANS LES RÉTINITES PIGMENTAIRES DOMINANTES AUTOSOMIQUES

C.P. Hamel (1, 2), B. Bocquet (2), D. Coustes-Chazalette (1), C.M. Dhaenens (3), D. Allorge (3), C. Titah (1), M. Hébrard (2), I. Meunier (1, 2), G. Manès (2)

(1) Centre de référence maladies sensorielles génétiques, CHRU Montpellier, France; (2) INSERM U583, Montpellier, France; (3) Laboratoire des génopathies, CHRU Lille, France

Contact : christian.hamel@inserm.fr

BUT : Les rétinites pigmentaires dominantes autosomiques (adRP) sont des dystrophies héréditaires de la rétine caractérisées par la perte progressive des photorécepteurs prédominante en périphérie rétinienne. La prévalence de cette affection est d'environ 1 individu sur 12000 . Les études précédentes suggèrent que dans moins d'un cas sur deux $(44,7 \%)$, une mutation est identifiée dans l'un des 16 gènes actuellement connus. Le but 
de cette étude est d'établir la prévalence réelle en France des gènes connus et de localiser de nouveaux gènes ou loci à partir de 250 familles recrutées de 2009 à 2012. MÉTHODES : Les études de liaison indirecte ont été effectuées avec 32 marqueurs de microsatellites encadrant $15 / 16$ gènes d'adRP (PRPF31, RP1, IMPDH1, PRPF8, NRL, PRPF3, CA4, RDS, CRX, TOPORS, NR2E3, KLHL7, ROM1, FSCN2 et PAP1) plus RHO qui est séquencé systématiquement. Pour certaines familles, une analyse du génome entier par puce Affymetrix ${ }^{\$ 2} 250 \mathrm{~K}$ a été réalisée et les résultats analysés, par trios parents-enfants ou sur l'ensemble de la famille en comparant les SNPs des patients atteints entre eux, grâce à un logiciel conçu dans notre équipe. Des régions chromosomiques candidates ont ainsi été délimitées et les gènes localisés ont ensuite été séquencés. RÉSULTATS : La stratégie comporte une première étape de criblage des 8 gènes les plus fréquemment mutés dans les adRP (RHO, PRPF31, RDS, RP1, PRPF8, IMPDHI, NRL et PRPF3) qui est en cours, permettant théoriquement d'identifier la mutation causale dans $38,7 \%$ des patients. Une étude indirecte réalisée sur 13 familles a permis d'identifier 5 mutations déjà répertoriées dans les bases de données: RHO p.Ala333fsX22 ( 1 famille) et p.Asn15Ser ( 2 familles), NR2E3 p.Gly56Arg ( 3 familles), RDS p.Ser198Arg ( 1 famille), RPGR p.Trp669X ( 1 famille), et 4 nouvelles mutations : PRPF31 p.Gly233ValfsX17 (1 famille) et p.[Pro217Thr] + [Asn218Lys] (1 famille), RDS p.Leu254Gln ( 2 familles) et RP1 p.Tyr685X (1 famille). Une analyse du génome entier a été faite pour 2 familles. Dans la première, un locus de $15 \mathrm{Mb}$ sur le chromosome 8 a permis d'identifier une nouvelle mutation dans RP1, p.lle725fsX737. Pour la seconde famille, un locus de $52,9 \mathrm{Mb}$ sur le chromosome 2 contenant 415 gènes a été isolé. L'étude et la caractérisation de ce locus est en cours. CONCLUSION : Les premiers résultats, dans cette cohorte restreinte, semblent souligner que la prévalence des gènes connus est probablement sous-estimée dans la littérature puisque la mutation causale a été identifiée pour 14 familles sur 15 , soit dans $93.3 \%$ des cas. Cependant, sur 15 familles étudiées, un nouveau locus a déjà été identifié pour lequel le gène est activement recherché.

Mots-clés : rétinite pigmentaire, génotypage de SNP.

TP483. IDENTIFICATION DE DEUX NOUVELLES DÉLÉTIONS ET D'UNE DUPLICATION DU GËNE OCA2 CHEZ DES PATIENTS ATTEINTS D'ALBINISME OCULOCUTANÉ

E. Lasseaux (1), C. Rooryck-Thambo (1), C. Plaisant (1), H. Dollfus (2), S. Defoort-Dhellemmes (3), B. Duban-Bedu (4), T. J.L. De Ravel (5), D. Lacombe (1), B. Arveiler (1)

(1) Service de Génétique Médicale, CHU de Bordeaux, France ; (2) Service de Génétique Médicale, CHU de Strasbourg, France; (3) Service Exploration fonctionnelle de la vision, CRHU de Lille, France; (4) Centre de Génétique Chromosomique, Hôpital Saint Vincent de Paul Lille, France ; (5) Centrum Menselijke Erfelijkheid, Leuven, Belgique Contact : eulalie.lasseaux@chu-bordeaux.fr

L'albinisme oculocutané ( $\mathrm{AOC}$ ) est une maladie génétique rare (1 personne sur 20000 environ) due à un déficit de synthèse de la mélanine et caractérisée par une hypopigmentation généralisée de la peau, des cheveux et des yeux, et par des anomalies ophtalmologiques. L'AOC est autosomique récessif et génétiquement hétérogène. On distingue quatre type d'AOC: $\mathrm{AOC1}$ dû à des mutations du gène TYR localisé en $11 \mathrm{q} 14.3$, $\mathrm{AOC} 2$ dû à des mutations du gène $\mathrm{OCA} 2$ (ou gène $\mathrm{P}$ ) en $15 \mathrm{q} 11.2$-q12, $\mathrm{AOC} 3$ dû à des mutations de TYRP1 en $9 \mathrm{p} 23$ et AOC4 dû à des mutations de SLC45A2 en 5p13.3. La forme la plus fréquente d'AOC est l'AOCI ( $46 \%$ des cas). L'AOC2 constitue la seconde forme d'AOC ( $29 \%$ des cas). Le gène $\mathrm{OCA} 2$ contient 25 exons et couvre $345 \mathrm{~kb}$. Plus de 80 mutations et autant de polymorphismes de type SNP ont été décrits dans ce gène (Human Gene Mutation Database of Cardiff, http ://www.hgmd.org/ : Albinism database, http ://albinismdb.med.umn.edu/). Plusieurs délétions intragéniques ont été décrites. On citera la délétion récurrente de $2,7 \mathrm{~kb}$ emportant l'exon 7 retrouvée chez les patients d'origine Africaine ou Afroaméricaine et la délétion de $122,5 \mathrm{~kb}$ spécifique des indiens Navajos. L'analyse du gène OCA2 dans notre laboratoire est effectuée par DHPLC ou PCR-HRM et séquençage à la recherche de mutations ponctuelles et par QMF-PCR à la recherche de réarrangements intragéniques. Nous décrivons ici deux nouvelles délétions et une duplication intragéniques, découvertes après étude du gène OCA2 chez 37 patients. Une délétion (Del1) emportant les exons 2 à 20 a été trouvée chez un patient à l'état hétérozygote, associée à une mutation ponctuelle dans l'exon 9 (c.1001C >T/p.Ala334Val). Une seconde délétion (Del2) emportant les exons 3 à 20 a été mise en évidence chez une patiente à l'état hétérozygote, et chez une autre patiente à l'état homozygote. Ces patientes sont toutes deux d'origine polonaise. Une duplication (Dup) a été trouvée à l'état homozygote chez une patiente. L'intervalle concerné par cette duplication débute après la Del2 dans l'intron 2 et termine dans la même région de 1'intron 20 que la Del2. Le caractère récurrent de la Del 2 chez deux patients dont un homozygote, et le caractère homozygote de la Dup chez une patiente nous ont interrogés quant aux mécanismes impliqués. Un éventuel effet fondateur a été investigué par analyse d'haplotype pour chacun de ces deux évènements. L'existence de séquences favorisant ces remaniements génomiques (éléments répétés, duplications segmentaires,...) a été analysée par bioinformatique. Les résultats seront présentés.

Mots-clés : albinisme oculocutané, OCA2, délétion/duplication.

IP484. ET S'IL S'EN FALLAIT DE QUELQUES BASES POUR QU'UN RETARD MENTAL S'ASSOCIE À UNE RÉTINOPATHIE PIGMENTAIRE LIÉE À L'X ?

N. Delphin (1, 2), J. Bengoa (2), S. Gerber (1), R. Borghese (2), A. Driben (2), S. Gobin (2), J.P. Bonnefont (2), J.M. Rozet (1), J. Kaplan (1, 2)

(1) Unité INSERM U78I \& Université Paris Descartes, CHU NeckerEnfants Malades, Paris, France : (2) Laboratoire Hospitalier de Génétique Moléculaire, CHU Nzcker-Enfants Malades, Paris, France Contact : josseline.kaplan@inserm.fr

Les rétinopathies pigmentaires liées au chromosome $\mathrm{X}$ figurent parmi les plus sévères des dystrophies rétiniennes affectant l'enfant et entrainant une grave dysfonction visuelle avant l'âge de 30 ans. Deux gènes rendent compte de la quasi-totalité des cas: RPGR ( $70 \%$ des cas) et RP2 (30\% des cas). Par ailleurs, il a été décrit dans deux familles indépendantes un syndrome délétionnel de la région Xp11.3 emportant les premiers exons du gène RP2 et s'étendant sur $1,2 \mathrm{Mb}$ vers la région télomérique du bras court. Dans ces deux familles, la rétinopathie pigmentaire s'associait à un retard mental suggérant un syndrome de gènes contigus. Par le criblage des gènes contenus dans la délétion chromosomique dans des familles atteintes de retard mental lié à l'X non-syndromique, le gène ZNF674 s'est retrouvé au banc des accusés par l'identification de trois mutations, dont une nulle. Nous rapportons ici, deux familles de rétinopathie pigmentaire liée à l'X rassemblant respectivement 7 et 2 garçons atteints. Aucun de ces patients ne souffrait d'un retard mental associé à leur déficience visuelle. Une étude génétique a permis d'incriminer la région RP2 dans ces deux familles et montrait une probable délétion de la totalité du gène RP2 ainsi que de marqueurs polymorphes flanquant la région 5'du gène. La présence du gène ZNF674 a été recherchée en priorité sous l'hypothèse de sa responsabilité dans le retard mental du syndrome délétionnel Xp11.3. Des couples d'amorces permettant d'amplifier spécifiquement les région 5 'et 3'UTR du gène ZNF674 dont il faut souligner l'orientation tête-bêche par rapport à RP2 ont permis de montrer l'absence du 3'UTR avec conservation de la région 5 '. Compte tenu de l'orientation du gène, ces dernières données suggèrent l'existence d'une boucle de délétion avec un point de cassure dans la séquence du gène ZNF674. Le point de cassure est en cours d'identification mais il est vraisemblable que les patients des deux familles présentées ici sont «passés à quelques bases près " d'un retard mental associé à leur rétinopathie pigmentaire.

Mots-clés: rétinopathie pigmentaire liée à l'X, syndrome délétionnel Xp11.3, gènes RP2-ZNF674.

P485. ÉTUDE CLINIQUE ET GÉNÉTIQUE D'UNE FAMILLE TUNISIENNE CONSANGUINE ATTEINTE DE BLÉPHAROPHIMOSIS PTOSIS EPICANTHUS INVERSUS ET TÉLÉCANTHUS

I. Chouchene (1, 2), K. Derouiche (1), A. Chaabouni (3), L. Cherif (2), A. Amouri (4), L. Largueche (1), S. Abdelhak (2), L. El Matri (1) (I) Unité de recherche d'Oculogénétique, Institut Hedi Rais d'Ophtalmologie, Tunis, Tunisie; (2) Unité de recherche «Investigation Moléculaire de maladies orphelines d'origine génétique », Institut Pasteur de Tunis, Tunisie ; (3) Service B, Institut Hedi Rais d'Ophtalmologie, Tunis, Tunisie ; (4) Laboratoire de Cytogénétique, Institut Pasteur de Tunis, Tunisie Contact : chouchen_ibtissem@yahoo.fr

Le syndrome de blépharophimosis ptosis épicanthus in versus et telecanthus (BPES) est une maladie oculaire rare qui se manifeste par une malformation des paupières qui peut être associée avec une prématurité ovarienne (BPES type I) ou non (BPES type II). La transmission est autosomique dominante. Les mutations responsables de cette pathologie touchent le gène FOXL2 $(3 \mathrm{q} 23)$ dans $80 \%$ des malades. Ce gène est un facteur de transcription qui s'exprime dans le mésenchyme des paupières en cours du développement embryonnaire et dans les follicules. Les mutations du gène FOXL2 sont essentiellement intragéniques $(81 \%)$. Une étude clinique chez une famille Tunisienne consanguine recrutée à l'Institut Hedi Rais d'Ophtalmologie de Tunis a permis de diagnostiquer cinq membres atteints de la famille. Afin d'identifier la mutation, nous avons effectué un séquençage direct du gène FOXL2. L'analyse de la séquence a identifié la nouvelle mutation g. 1113 dup C, c.876 dup C, p.P292 Fs. Cette mutation 
a causé un changement de cadre de lecture et l'extension de la protéine à 532 acides aminés. Cette étude a rapporté pour la première fois une nouvelle mutation chez deux générations d'une famille Tunisienne atteinte de BPES. Notre résultat étend le spectre mutationnel du gène FOXL2.

Mots-clés: syndrome de blépharophimosis, gène FOXL2, nouvelle mutation.

P486. MUTATION IVS1+1G >A DU GÈNE CHM RESPONSABLE DE LA CHOROÏDÉRÉMIE DANS UNE FAMILLE TUNISIENNE I. Ben Charfeddine (1), M. Gribaa (1), N. Ben Rayana (2), L. Adala (1), O. Mammaï (1), A. Mili (1), T. Belazreg (1), A. Amara (1), F. Ben Hadj Hamida (2), A. Saad (1)

(1) Laboratoire de Cytogénétique, de Génétique Moléculaire et de Biologie de la Reproduction Humaines, CHU Farhat Hached, Sousse, Tunisie ; (2) Service d'Ophthalmologie, CHU Farhat Hached, Sousse, Tunisie Contact : moez.gribaa@rns.tn

La choroïdérémie (CHM) est une maladie héréditaire rare à transmission récessive liée au chromosome X. Elle est caractérisée par une dystrophie de l'épithélium pigmentaire de la rétine. Cliniquement, elle se manifeste par une perte de la vision nocturne et une constriction progressive du champ visuel entraînant une cécité dans le jeune âge. Elle est causée par la mutation du gène $\mathrm{CHM}$. Les femmes hétérozygotes porteuses ont généralement une fonction rétinienne normale mais présentent, au fond d'œil, des zones de dégénérescence et de pigmentation focales de la rétine. Ces zones sont en fait des régions pathologiques dues à l'inactivation aléatoire du chromosome X. Dans ce travail, nous rapportons l'étude de 3 patients atteints de CHM et faisant partie d'une même famille tunisienne. Ces patients ont consulté pour une perte de la vision nocturne. Le fond d'œil a montré une atrophie chorio-rétinale diffuse. Les 3 patients ont aussi présenté une réduction du champ visuel et l'absence de signal à l'électrorétinogramme (ERG). Le séquençage direct des différents exons du gène $\mathrm{CHM}$ et des régions introniques qui les entoure, a permis de retrouver la transition d'une guanine $(G)$ par une adénine $(A)(G>A)$ dans le site donneur d'épissage de l'intron 1 (IVS1+1G $>A$ ) à l'état hémizygote chez les 3 patients. Cette mutation est à l'origine d'un épissage aberrant de l'ARNm entrainant l'apparition d'un codon stop prématuré et une protéine REP-1 tronquée qui a perdu toute fonctionnalité.

Mots-clés : gène CHM, choroïdérémie, protéine REP-1.

IP487. GÈNE PDE6C, NOUVEAU LOCUS POUR L'ACHROMATOPSIE COMPLÈTE AUTOSOMIQUE RÉCESSIVE

Y. Perdomo-Trujillo (1), V. Pelletier (1), T. Grau (2), H. Dollfus (1), B. Wissinger (2), S. Kohl (2)

(1) Centre de Référence pour les Affections Rares en Génétique Ophtalmologique (CARGO) et Service de Génétique Médicale, Hôpitaux Universitaires de Strasbourg, Strasbourg, France ; (2) Molecular Genetic Laboratory, Institute for Ophthalmic Research, Department for Ophthalmology, University Clinics Tübingen, Germany

Contact : yauemprend@yahoo.fr

L'Achromatopsie complète constitue une maladie congénitale et stationnaire. Elle se transmet sur le mode récessif autosomique. Cliniquement, elle est caractérisée par la présence d'une importante photophobie, d'un nystagmus, d'une acuité visuelle basse, associés à une absence complète de vision colorée. À l'électrorétinogramme, les réponses du système des bâtonnets sont présentes. Les réponses correspondant au système des cônes sont très diminuées, voire absentes. Une dysfonction des trois populations de cônes caractérise cette maladie génétique. Trois gènes sont impliqués dans l'Achromatopsie complète : CNGA3, CNGB3 et GNAT2. Ici nous montrons le cas d'une famille consanguine, porteuse d'une mutation homozygote c.1682->1683insA/c.1682->1683insA, dans le gène PDE6C. Ce gène a été récemment identifié à l'origine de cette maladie par le groupe de Tübingen. PDE6C code pour une protéine spécifique des cônes, la cGMP phosphodiesterase alpha', qui constitue un élément clé dans la cascade de la phototransduction des cônes.

Mots-clés : achromatopsie complète, autosomique récessive, PDE6C.

IP488. PREMIÈRE OBSERVATION MAROCAINE D'AMAU. ROSE CONGÉNITALE DE LEBER SECONDAIRE À LA MUTATION C.1511_1514 DEL GAGA DU GENE CEP290

N. Aboussair (1), S. Chafai Elalaoui (1), I. Cherkaoui Jaouad (1), L. Rifai (1), J.M. Rozet (2), J. Kaplan (2), A. Sefiani $(1,3)$

(I) Département de Génétique Médicale, Institut National d'Hygiène, Rabat, Maroc ; (2) Unité de Génétique et Épigénétique des maldies métaboliques neuroensorielles et du développement, Hôpital Necker Enfants médecine et de pharmacie de Rabat, Université Mohammed V, Souissi, Rabat, Maroc

Contact : nisrineaboussair@yahoo.fr

L'amaurose congénitale de Leber (ACL, MIM 204000) est la plus précoce et la plus sévère des dystrophies rétiniennes héréditaires puisqu'elle est responsable de cécité ou de malvoyance profonde néonatale ou périnatale (Leber T, 1869). Ce qui caractérise avant tout cette affection est que le fond d'œil est normal à la naissance contrastant avec un électrorétinogramme (ERG) éteint témoignant d'une perte totale de la fonction des cônes et des bâtonnets. L'ACL est une maladie héréditaire à transmission autosomique récessive. Néanmoins, quelques cas d'ACL à transmission autosomique dominante ont été rapportés dans la littérature. Cette maladie est caractérisée par une grande hétérogénéité génétique et physiopathologique. En effet à ce jour 11 gènes, impliqués dans des voies physiologiques différentes et 2 loci LCA 3 en $14 \mathrm{q} 24$ et LCA9 en 1 p36 ont été rapportés. Des études récentes ont montré que le gène NPHP6 (CEP290) est impliqué dans $22 \%$ des cas d'ACL. Nous rapportons dans ce travail, l'observation d'une patiente Marocaine, âgée de 2 ans, issue d'un mariage consanguin et présentant une cécité congénitale associée à une photophobie et à un retard psychomoteur. L'examen ophtalmologique et les examens électrophysiologiques ont évoqué une dégénérescence tapétorétinienne. Une étude indirecte par la méthode de cartographie par homozygotie a mis en évidence une liason au gène CEP290. Par la suite le séquençage du gène CEP290 a identifié une mutation homozygote c.1511_1514 del GAGA. En conclusion, l'identification de cette mutation nous a parmis de confirmer le diagnostic d'amaurose congénitale de Leber chez cette patiente, de formuler un conseil génétique adéquat pour les parents ainsi que de proposer un diagnostic anténatal pour les grossesses ultérieures.

Mots-clés : amaurose congénitale de leber, CEP290, mutation.

DP489. ÉTUDE DU GÈNE FOXII DANS LES SURDITÉS RÉCESSIVES NON SYNDROMIQUES AVEC LARGE AQUEDUC VESTIBULAIRE

A. Buffet, V. Gaston, D. Dupin-Deguine, P. Calvas, E. Bieth

Service de génétique, Hôpital Purpan, Toulouse, France

Contact : alexbuffet13@yahoo.fr

Les surdités récessives non syndromiques de type DFBN4 et le syndrome de Pendred ont en commun des malformations de l'oreille interne incluant de larges aqueducs vestibulaires (LAV) et parfois une malformation de Mondini. Ces surdités sont connues pour être liées à des mutations du gène SLC26A4. Toutefois, chez environ 1/3 des patients présentant un phénotype indistinguable aucune mutation n'est retrouvée dans ce gène $[1,2]$. Ce constat soulève la question d'une hétérogénéité génétique. Ainsi, le gène codant le facteur de transcription FOXIl contrôlant l'expression de SLC26A4 constituerait un candidat potentiel. Une étude récente a d'ailleurs rapportée des mutations hétérozygotes de ce gène chez des patients malentendants avec au scanner des rochers des anomalies de type LAV [3]. Nous avons sélectionnés 22 patients présentant ce phénotype et chez lesquels aucune mutation du gène SLC26A4 n'a été détectée. La recherche de mutations ponctuelles (séquençage) et de grands réarrangements (QMPSF) du gène FOXII a été entreprise. Il s'avère qu'aucune anomalie de FOXII n'a été observée chez les 22 patients analysés. En conclusion, nos résultats sur cette petite cohorte suggèrent que le gène FOXII est probablement rarement impliqué dans la genèse des surdités avec LAV.

\section{Références}

1. Campbell C., Hum. Mutation 2001 ; $17(5): 403-11$.

2. Gonzalez T., Eur. J. Endocrinology $2001 ; 144(6): 585-93$.

3. Yang T., Am. J. Hum. Genet. $2007: 80: 1055-63$.

Mots-clés : foxil, pendred.

\section{DP490. SURDITÉ AVEC LARGES AQUEDUCS VESTIBULAIRES ET MUTATION HÉTÉROZYGOTE DU GÈNE SLC26A4: RECHERCHE DE GRANDS RÉARRANGEMENT DE SLC26A4 ET} D'UN DIGÉNISME IMPLIQUANT LE GENE FOXII

A. Buffet, V. Gaston, D. Dupin-Deguine, P. Calvas, E. Bieth

Service de génétique, Hôpital Purpan, Toulouse, France

Contact : alexbuffet13@yahoo.fr

Les malformations de l'oreille interne de type large aqueduc vestibulaire (LAV) sont connues pour être associées au syndrome de Pendred (PDS et à la surdité non syndromique de type DFBN4. Ces deux types de surdité récessive ont en commun d'être liées à des mutations du gène SLC26A4. Toutefois, chez environ $30 \%$ des patients malentendants présentant des LAV, une seule mutation du gène SLC26A4 est détectée à l'état hétérozygote [1,2], ceci malgré un criblage exhaustif des 21 exons, des jonctions introniques et du promoteur du gène. Cette situation génère des interrogations sur le plan diagnostique et est source de difficultés pour le conseil génétique. Nous avons développé une méthode de PCR semi-quantitative 
(QMPSF) pour identifier chez ces patients hétérozygotes de larges remaniements du gène SLC26A4 indétectables par les méthodes de séquençage. Au total, 9 patients ont été analysés et aucune délétion ou duplication n'a été retrouvée sur les exons testés du gène SLC26A4. Par ailleurs, dans l'hypothèse d'un éventuel digènisme impliquant le gène FOXII [3] nous avons recherché chez ces 9 patients des mutations et des remaniements dans ce gène candidat. Les résultats de ces analyses se sont également avérés négatifs. En conclusion, nous n'avons pas pu mettre en évidence de grand remaniement du gène SLC26A4 dans notre petite cohorte. D'autres études sont nécessaires pour préciser l'implication du gène SLC26A4 chez les patients malentendants et hétérozygotes: association fortuite ou autre mutation non détectée ou encore digénisme (implication putative du gène FOXI1 ou plus récemment de KCNJ10) ?

\section{Références}

1. Campbell C., Hum. Mutation $2001 ; 17(5): 403-11$.

2. Gonzalez T., Eur. J. Endocrinology $2001 ; 144(6): 585-93$.

3. Yang T., Am. J. Hum. Genet. 2007; 80: 1055-63.

Mots-clés : pendred, qmpsf.

IP491. LA FIÈVRE QUI REND SOURD !

S. Marlin (1, 2, 3), Y. Nguyen (4), N. Loundon (2, 4), I. Rouillon $(2,4)$, E.N. Garabedian $(2,3,4)$, L. Jonard $(2,3,5)$, F. Denoyelle $(2,3,4)$, D. Feldmann $(2,3,5)$

(1) Service de Génétique, Hôpital Trousseau, APHP, Paris, France ; (2) Centre de Références des Surdités Génétiques, Hôpital Trousseau, APHP, Paris, France ; (3) Unité INSERM U587, Hôpital Trousseau, APHP, Paris, France; (4) Service d'ORL pédiatrique, Hôpital Trousseau, APHP, Paris, France; (5) Service de Biochimie et Biologie Moléculaire, Hôpital Trousseau, APHP, Paris, France

Contact : sandrine.marlin@trs.aphp.fr

La neuropathie auditive/désynchronisation auditive (NA/DA) est une altération de l'audition associant un trouble de la synchronisation du nerf auditif avec une conservation de l'activité des cellules ciliées externes. Le site suspecté de la lésion peut être soit la synapse entre la cellule ciliée interne et le nerf cochléaire soit le nerf cochléaire lui-même. Le diagnostic est réalisé devant la présence d'oto-émissions acoustiques associées à des potentiels évoqués acoustiques (PEA) absents ou désynchronisés. Bien que des causes génétiques et environnementales aient été identifiées la majorité des cas restent inexpliqués. Nous présentons le cas d'une famille consanguine composée d'une fratrie de deux sœurs et d'un frère. En condition normale (sans fièvre) les patients présentent une audition normale ou une surdité de perception légère bilatérale avec une compréhension vocale altérée. En condition fébrile et en dehors de toute affection ORL, les trois enfants présentent une surdité de perception sévère à profonde associée à un effondrement de la compréhension vocale. Le déficit auditif est réversible avec le retour à l'apyrexie. Les examens audiométriques montrent la présence des oto-émissions acoustiques avec une absence d'onde individualisable sur les PEAs. Le bilan d'imagerie n'a pas retrouvé d'anomalie morphologique de la cochlée ou du paquet acoustico-facial. Une nouvelle mutation du gène OTOF a été identifiée à l'état homozygote chez les trois enfants. Le gène OTOF a été antérieurement identifié dans des cas de surdité profonde congénitale associée à la conservation initiale des otoémissions acoustiques. L'otoferline est une protéine exprimée uniquement dans les cellules ciliées internes et est impliquée dans le trafic et la fusion calcium dépendante des vésicules présynaptiques. Ce travail nous permet de définir un nouveau phénotype de neuropathie auditive et d'identifier une nouvelle mutation thermosensible chez l'homme.

Mots-clés : neuropathie auditive, otoferline, mutation thermosensible.

\section{IP492. NOUVELLES MUTATIONS DU GÈNE WFS1 DANS LES SURDITÉS ISOLÉES AVEC COURBE AUDIOMÉTRIQUE ASCENDANTE (ATTEINTE PRÉFÉRENTIELLE DES FRÉ- QUENCES GRAVES) OU EN U}

L. Jonard (1), M. Niasme (1), C. Bonnet (1), M. Louha (1), C. Koval (1), D. Feldmann (1), R. Couderc (1), H. Dollfus (2), V. Drouin-Garraud (3), D. Lacombe (4), F. Fellmann (5), C. Francannet (6), G. Lina-Granade (7), F. Denoyelle (8), S. Marlin (9)

(1) Laboratoire de Biochimie, Inserm U587, Hôpital Armand-Trousseau, AP-HP, Paris, France; (2) Service de Génétique Médicale, Hôpital de Hautepierre, Strasbourg, France; (3) Unité de Génétique Clinique, Hôpital Charles-Nicolle, Rouen. France; (4) Service de Génétique, Hôpital Pellegrin, Bordeaux, France; (5) Service de Génétique, Hôpital Saint-Jacques, Besançon, France; (6) Génétique Médicale, Hôtel-Dieu, Clermont-Ferrand, France ; (7) Service ORL, Hôpital Édouard-Herriot, Lyon, France; (8) Service d'ORL et de Chirurgie Cervico-Faciale, Inserm U587, Hôpital Armand-Trousseau, AP-HP, Paris, France; (9) Unité de
Génétique Médicale, Inserm U587, Hôpital Armand-Trousseau, AP-HP, Paris, France.

Contact : laurence.jonard@trs.aphp.fr

Introduction : Le syndrome de Wolfram ou DIDMOAD (pour Diabetes Insipidus, Diabetes Mellitus, Optic Atrophy and Deafness) est une surdité syndromique de transmission autosomique récessive causée par des mutations du gène WFS1. Parallèlement à son implication dans le syndrome de Wolfram, des mutations dominantes de WFS1, siégeant principalement dans l'exon 8 du gène et affectant l'extrémité C-terminale de la protéine, wolframine, sont retrouvées dans des formes non syndromiques de surdité avec atteinte préférentielle des fréquences graves (surdité DFNA6/14/38). Méthodes: Nous avons analysé le gène WFS1 dans une large cohorte de cas sporadiques ou familiaux de surdité non syndromique avec courbe audiométrique ascendante (atteinte préférentielle des fréquences graves) ou courbe en U. Résultats: Douze nouvelles mutations hétérozygotes du gène WFS1 (11 mutations ponctuelles faux-sens et une duplication) ont été identifiées. Certaines d'entre elles ont été observées dans plusieurs familles. Nous avons également pu montrer leur présence dans des formes « frontières " de surdité autosomique dominante associée à une atteinte ophtalmologique (atrophie optique) ou à un diabète sucré. Conclusion: L'étude moléculaire du gène du syndrome de Wolfram, WFS1, trouve sa pertinence dans le diagnostic génétique des surdités non syndromiques, sporadiques ou familiales, avec courbe audiométrique ascendante ou en U. Il faut également l'évoquer dans les formes frontières autosomiques dominantes associant une surdité et une atrophie optique, ou une surdité et un diabète.

Mots-clés : surdité dominante, WFS1, DFNA6.

\section{P493. CHARACTERIZATION OF THE MOLECULAR OVERLAP BETWEEN WAARDENBURG SYNDROME TYPE 2 AND 4}

V. Pingault (1, 2, 3), F. Dastot-Le Moal (1, 3), D. Ente (3), T. Attie-Bitach (4), R. Touraine (5), J. Amiel (4), M. Goossens (1, 2, 3), S. Marlin (6), N. Bondurand $(1,2)$

(I) INSERM U841, IMRB, Département de génétique, Equipe 11, Créteil, France: (2) Université Paris 12, Faculté de Médecine, IFR10, Créteil, France : (3) AP-HP, Groupe Albert Chenevier-Henri Mondor, Service de biochimie et génétique, Créteil, France; (4) INSERM U78I, Hôpital Necker, AP-HP, Paris, France ; (5) CHU-Hôpital Nord. Service de Génétique, Saint Etienne, France: (6) Service de Génétique, Centre de référence "Surdités génétiques", INSERM U587, Hôpital Armand-Trousseau, France

Contact : veronique.pingault@hmn.aphp.fr

Waardenburg syndrome (WS) is a rare $(1 / 40000)$ auditory-pigmentary disorder that exhibits varying combinations of sensorineural hearing loss and abnormal pigmentation of the hair and skin. Depending on additional symptoms, WS is classified into four subtypes, WS1 to 4. Absence of additional features characterizes WS2. The association of facial dysmorphic features defines WSI and 3 whereas the association with Hirschsprung disease (aganglionic megacolon) characterizes WS4, also called Waardenburg-Hirschsprung disease. Mutations within the genes encoding MITF and SNAI2 have been identified in $15 \%$ of WS2 cases, mutations within the PAX3 gene explain almost all WS1 and WS3 cases, whereas mutations of EDN3, EDNRB and SOX10 are responsible of $65-85 \%$ of WS4. Recently, we described the first SOX10 deletions in patients presenting with WS2. After the involvement of PAX 3 in WS1 and 3, the identification of SOX10 mutations in WS2 and 4 further documented the molecular complexity and close relationship that link the different subtypes of WS and invited to fully characterize the molecular overlap between WS2 and WS4. We took advantage of the point mutation detection systems routinely used in the laboratory, but also of other strategies such as QMF-PCR assay and looked for point mutations and rearrangements within the EDN3/EDNRB and MITF/SNAI2 genes in both $30 \mathrm{WS} 4$ and $30 \mathrm{WS} 2$ cases. We identified new mutations within the MITF, EDN3, and EDNRB genes. These comprehensive studies are necessary to fully document the complexity of WS, and show that the molecular overlap between WS2 and 4 mostly involves the SOX10 gene.

Mots-clés : Waardenburg syndrome, Deafness, SOX10.

- 4 494. ANALYSE DU GÈNE KRTHB5 DANS UNE LARGE FAMILLE TUNISIENNE DE DYSPLASIE ECTODERMIQUE « ONGLES-CHEVEUX » PURE

M. Trabelsi (2), S. Mansour (2), M. Trabelsi-Ouelha (1, 2), I. Chelly (1), Y. Elaribi (1), H. Jilani (1), I. Ouertani (1), L. Eleuch (1), F. Maazoul (1), Z. Marrakchi (3), M. Kharrat (2), H. Chaabouni (1, 2), R. M'rad (1, 2) (1) Service des maladies Congénitales et Héréditaires, EPS Charles Nicolle, Tunis, Tunisie; (2) Laboratoire de Génétique Humaine, Faculté 
de Médecine de Tunis, Tunisie ; (3) Service de Néonatalogie, EPS Charles Nicolle,Tunis, Tunisie

Contact : rmrad@yahoo.com

Les dysplasies ectodermiques (ED) définissent une famille d'anomalies du développement des annexes de la peau (ED) (cheveu, ongles, dents, glandes sudoripares. Deux cent cas de (ED) sont rapportés dans la littérature, dans environ 3 cas un gène a été associé au phénotype. Sur le plan clinique on différencie les formes de ED ongle cheveu pures des formes de ED associées à des malformations non ectodermiques. Naeem et al ont rapporté une large famille Pakistanaise de ED ongle cheveu pure secondaire à une mutation faux sens du gène KRTHB5 localisé en $12 \mathrm{q} 13.13$. Nous rapportons une large famille Tunisienne de ED ongle cheveu- pure comportant 5 sujets atteints et 3 phénotypiquement sains. La maladie se transmet selon le mode autosomique récessif dans cette famille. Une étude indirecte avec 5 marqueurs polymorphes liés au cluster des gènes de kératine type II retrouve une liaison. Le séquençage du gène KRTHB5, qui constitue le gène candidat le plus probable dans cette pathologie, retrouve une mutation de la région 3'UTR du gène. Cette mutation est retrouvée à l'état homozygote chez tous les patients et hétérozygote ou absente chez les sujets phénotypiquement sains. Ce résultat constitue le deuxième cas dans la littérature de mutation du gène KRTHB5 associé à une ED « ongles-cheveux » pure.

Mots-clés : keartine, dysplasie ectodermique, 3'UTR.

IP495. ÉPIDERMOLYSE BULLEUSE SIMPLEX DE DOWLING. MEARA : NOUVELLES MUTATIONS DES KÉRATINES 5 ET 14 ET CARACTÉRISATION PHÉNOTYPIQUE

A. Ervais Wakosa (1), A. Terron-Kwiatkowski (2), A. Charlesworth (3), C. Thauvin (4), E. Collet (1), S. Dalac-Rat (1), L. Faivre-Olivier (4), F. Huet (5), J.P. Lacour (3), D. Baty (2), P. Vabres (1)

(I) Service de Dermatologie, Centre Hospitalo-Universitaire, Dijon, France, (2) Molecular Genetics, Ninewells Hospital, Dundee, United Kingdom; (3) Centre de référence des épidermolyses bulleuses, Hôpital de l'Archet 2, Nice, France: (4) Service de Génétique, Centre Hospitalo-Universitaire, Dijon, France; (5) Service de Pédiatrie, Centre Hospitalo-Universitaire, Dijon, France Contact:wakosa.adeline@me.com

Les épidermolyses bulleuse simplex (EBS) sont de gravité variable. Certaines peuvent être responsables de manifestations graves en période néonatale et d'un handicap notable à l'âge adulte. Nous rapportons quatre observations dans trois familles d'EBS type Dowling-Meara (DM) dont la caractérisation moléculaire a permis d'identifier trois nouvelles mutations des gènes de kératine 5 et 14. Chez tous les patients les kératines 5 et 14 étaient normalement exprimées dans l'épiderme, avec un clivage au sein des kératinocytes de la couche basale. Deux nouvelles mutations hétérozygotes de KRT 5 ont été identifiées. Chez une patiente de 29 ans, il s'agissait d'une délétion de 96 nucléotides impliquant un site d'épissage dans l'exon 1 et entrainant la délétion de 22 acides aminés (AA) dans le domaine 1A de l'hélice alpha centrale (c.657_751del96). Elle était responsable d'un phénotype grave en période néonatale: détresse respiratoire, et dans l'enfance : retard staturo-pondéral et d'acquisition de la marche. Il persistait un handicap important à l'âge adulte: impotence fonctionnelle surtout lors de fortes chaleurs et atteinte génitale. L'autre mutation de KRT5 (c.1397G>C), une substitution dans le domaine 2B, entrainait des manifestations majeures à la naissance, avec un décollement cutanéo-muqueux étendu mais l'évolution dans l'enfance était favorable, avec à 3 ans des bulles herpétiformes et une kératodermie plantaire modérées n'ayant pas gêné l'acquisition de la marche. Une duplication hétérozygote KRT14 a été identifiée chez une femme de 30 ans et son fils, dans l'exon 6 , entraînant un décalage du cadre de lecture dans le domaine $2 \mathrm{~B}$ et la production de $115 \mathrm{AA}$ aberrants (c.1131dupT). Les bulles ne sont apparues qu'après un intervalle libre d'une semaine après la naissance. Chez la mère, les bulles et la kératodermie plantaire avaient gêné l'acquisition de la marche mais le handicap à l'âge adulte était mineur. Chez son fils, malgré plusieurs poussées bulleuses les premières années, ni les bulles ni la kératodermie plantaire n'ont eu de conséquences graves, mais l'atteinte génitale a entrainé un phimosis. Dans les EBS, plusieurs dizaines de mutations ont été rapportées sur les gènes KRT5 et KRT14, impliquant des régions protéiques fonctionnellement importantes pour l'assemblage des filaments intermédiaires de kératine. Toutefois les descriptions phénotypiques sont souvent succinctes. La délétion de $22 \mathrm{AA}$ du domaine $1 \mathrm{~A}$ rapportée ici a déjà été observée avec une mutation différente d'un site d'épissage. Elle était associée à un phénotype particulièrement grave qui n'avait pas été précédemment noté. Dans deux cas existait une atteinte génitale gênante, non recensée avec l'EBS-DM dans le registre américain des EB. Des mutations du domaine 2B entrainant un décalage du cadre de lecture ont été rapportées pour des phénotypes sévères. Ici, cette nouvelle mutation entraînait un phénotype modéré.La corrélation génotype-phénotype semble donc imprévisible.

Mots-clés : épidermolyse bulleuse simplex de Dowling-Meara, kératines
TP496. ÉTUDE MOLÉCULAIRE D'UNE COHORTE INTERNATIONALE DE 78 NOUVEAUX CAS DE SYNDROME DE NÉTHERTON : VARIABILITÉ PHÉNOTYPIQUE, RÉCURRENCE ET CLUSTERING DES MUTATIONS

C. Deraison (1), L. Lacaze-Buzy (1), E. Bourdon-Lanoy (2), A.M. Mazarguil (3), R. Tomb (4), C. Moss (5), A.C. Bursztejn (6), K. Valari (7), L. Kostyuchenko (8), C. De Die-Smulders (9), C. Bodemer (2), A. Hovnanian $(1,10,11)$

(1) INSERM, U563, Département de Génétique, Toulouse, France; (2) Service de Dermatologie, Hôpital Necker Enfants Malades, Centre de Référence MAGEC, Paris, France; (3) Service de Génétique, CHU Purpan, Toulouse, France; (4) Département de Dermatologie, Hôpital Dieu de France, Breyrouth, Liban ; (5) Département de Dermatologie, Birmingham Children's Hospital, Birmingham, Grande Bretagne ; (6) Service de Dermatologie, Hôpital Fournier, Nancy, France : (7) Département de Pédiatrie, Sophia Children's Hospital, Athène, Grèce; (8) Centre of Children Immunology, Lviv, Ukraine; (9) Départment de Génétique clinique, Hôpital de Maastricht, Maastricht, Pays-Bas ; (10) Services de Génétique et de Dermatologie, Hôpital Necker Enfants Malades, Paris, France ; (11) Université Paris V René Descartes, Paris, France

Contact : alain.hovnanian@inserm.fr

Le syndrome de Netherton est une des maladies les plus sévères de la kératinisation de l'enfant et du jeune adulte. C'est une maladie génétique cutanée rare (1 sur 100000 naissances), transmise sur le mode autosomique récessif, associant une érythrodermie desquamative congénitale, une anomalie spécifique des cheveux (trichorrhexis invaginata ou " cheveux bambous ») et une atopie sévère avec des taux d'IgE élevés. Le SN est du à des mutations du gène SPINK5, qui code un inhibiteur de protéases, LEKTI. La majorité des mutations de SPINK5 conduisent à l'apparition d'un codon stop prématuré et prédisent la perte d'expression de la protéine. L'absence de LEKTI, révélée par immunohistochimie sur biopsie cutanée, permet de confirmer le diagnostic. Nous rapportons l'étude moléculaire d'une cohorte internationale de 67 familles correspondant à 78 patients 48 mutations différentes ont été identifiées, dont 33 sont nouvelles. La plupart des patients présentent la triade clinique classique, certains patients n'expriment qu'une partie de ces manifestations ou sous une forme atypique. L'immunomarquage de LEKTI réalisé chez $59 \%$ des patients de la cohorte montre dans la majorité des cas une perte complète d'expression de la protéine dans les couches granuleuses de l'épiderme. Les mutations des deux allèles de SPINK5 ont été identifiées chez $69 \%$ des familles. L'identification de la seconde mutation est en cours chez $27 \%$ des familles. La recherche de mutation par séquençage de SPINK5 est restée négative chez trois patients. Les mutations non sens ou entraînant un décalage du cadre de lecture conduisant à un codon stop prématuré représentent la majorité des anomalies identifiées $(56 \%)$. Les mutations d'épissage comptent pour $42 \%$ des mutations identifiées. Une seule mutation faux sens, affectant le codon d'initiation, a été mise en évidence. $43,5 \%$ des mutations sont des délétions ou des insertions de petite taille, dont 14 sont nouvelles. $54,5 \%$ des mutations sont des substitutions conduisant à des mutations non sens ( $13 \%$, dont 3 sont nouvelles) ou des anomalies d'épissage. Cinq mutations récurrentes ont été identifiées chez des patients d'origine géographique différente : 153 delT chez 8 patients originaires de Turquie et des Balkans, 238insG chez 6 patients originaires d'Italie et de Grèce, 316delGA chez 3 pateints d'Allemagne et de France, $2240+1 \mathrm{G}>\mathrm{A}$ chez 4 patients des Pays-Bas et du Chili, et R853X chez 3 patients de France. Cette étude est la première base de données internationales de mutations du gène SPINK 5 dans le SN. Elle montre que $68 \%$ des mutations identifiées dans cette cohorte sont localisées dans les exons $3,4,5$ et les exons 22-27, ce qui permet de définir des régions prioritaires du gène à étudier pour accélérer l'identification des mutations. Elle montre aussi que la recherche de microrearrangement génique est nécessaire chez de rares patients $\mathrm{SN}$ dont le diagnostic est certain, mais sans mutation de SPINK5 identifiée par séquençage.

Mots-clés : syndrome de Netherton, diagnostic moléculaire, spectre de mutations.

\section{- P497. NOUVELLE STRATÉGIE MOLÉCULAIRE POUR LE DIA- GNOSTIC ÉTIOLOGIQUE DES ICHTYOSES}

L. Lacaze-Buzy (1), C. Deraison (1), A. Hovnanian (1, 2, 3)

(1) INSERM, US63, Département de Génétique, Toulouse, France ; (2) Services de Génétique et de Dermatologie, Hôpital Necker Enfants Malades, Paris, France; (3) Université Paris V René-Descartes, Paris, France

Contact : alain.hovnanian@inserm.fr

Les ichtyoses héréditaires $(\mathrm{IH})$ forment un groupe hétérogène de génodermatoses rares et sévères. Elles présentent un large spectre clinique allant 
de l'ichtyose lamellaire (IL) caractérisée par de larges squames épaisses et brunes sans érythème, à l'Érythrodermie Congénitale Ichyosiforme (ECI) qui associe une érythrodermie et de fines squames blanches diffuses. À ce jour, six gènes différents ont été identifiés, sans que la présentation clinique ne permette d'impliquer l'un de ces gènes en particulier: TGM1, ABCA12, ALOX12B, ALOXE3, 1'ICHTHYIN et CYP4F22. Les outils de diagnostic immunohistochimique ou enzymologique sont actuellement limités à la transglutaminase 1, et l'analyse moléculaire systématique par séquençage de ces 6 gènes est longue et coûteuse, parfois incompatible avec les délais imposés par un diagnostic prénatal. Nous avons développé une nouvelle stratégie afin de répondre à ces difficultés. Celle-ci tient compte d'une part de la structure de la famille sollicitant un diagnostic d'ichtyose (notion et degré de consanguinité des parents, nombre de membres atteints dans la famille), et d'autre part des connaissances actuelles sur les spectres des mutations de ces gènes. Ainsi, après exclusion d'une IH par déficit en transglutaminase 1 (immunodétection et/ou activité enzymatique in situ normale(s) sur biopsie cutanée), l'analyse indirecte, reposant sur le génotypage de marqueurs génétiques de ces 6 gènes, est appliquée dans les familles consanguines (recherche d'homozygotie chez les sujets atteints) et dans les familles multiplexes (recherche de génotypes identiques entre membres atteints de la même famille). Si cette analyse est positive, les mutations seront recherchées par séquençage dans le ou les gènes candidats. Si l'analyse indirecte exclut les gènes testés, la maladie implique un gène encore non identifié. La seconde approche est complémentaire ou se substitue à la précédente dans les familles non consanguines n'ayant qu'un seul enfant atteint. Elle se base sur la recherche des points chauds de mutations correspondant à des mutations récurrentes retrouvées dans plusieurs familles non apparentées, ou à des effets fondateurs observés dans les familles de même origine géographique. Cette analyse a permis de sélectionner 13 exons (sur les 117 exons codants qui composent l'ensemble des 6 gènes) dont le séquençage permet d'identifier plus de $70 \%$ des mutations connues. Si aucune mutation n'est identifiée, le séquençage de toute la partie codante de ces gènes est discuté en fonction de la justification médicale de l'analyse. En conclusion, la combinaison de l'analyse génétique indirecte et directe par recherche des mutations récurrentes permet de réduire, de façon significative, les coûts liés au séquençage systématique des 6 gènes mais surtout de réduire considérablement les délais d'attente du diagnostic et du conseil génétique délivrés au patient et à sa famille.

Mots-clés : ichtyoses, stratégies d'investigation, diagnostic moléculaire.

qP498. BASES MOLÉCULAIRES DE L'ÉPIDERMOLYSE BULLEUSE SIMPLE DANS UNE COHORTE NATIONALE DE 31 PATIENTS : MUTATIONS NOUVELLES ET RÉCURRENTES DES KÉRATINES 5 , ET 14 ET NOUVELLES CORRÉLATIONS GÉNOTYPE-PHÉNOTYPE

V. Pendaries (1), L. Lacaze-Buzy (1), E. Bourdon-Lanoy (2), M. Titeux (1), S. Hadj-Rabia (2), S. Barbarot (3), A. David (3), P. Edery (4), M. Holder (5), N. Philip (6), Y. Sznajer (7), A. Toutain (8), S. Fraitag (9), C. Bodemer (2), A. Hovnanian $(1,10,11)$

(1) Inserm, U563, Département de Génétique, Toulouse, France ; (2) Service de Dermatologie, Hôpital Necker Enfants Malades, Centre de Reférence MAGEC, Paris, France; (3) Génétique Médicale, Hôpital de la Mère et de l'Enfant, Nantes, France; (4) Hôpital Debrousse, Unité de Génétique Médicale, Lyon, France; (5) Service de Génétique Clinique, Hôpital Jeanne-de-Flandre, Lille, France: (6) Service de Génétique, Hôpital d'enfants de la Timone, Marseille, France; (7) Laboratoire de Génétique Médicale, Hôpital Erasme, Bruxelles, Belgique ; (8) Service de Génétique, Hôpital Bretonneau, Tours, France; (9) Service d'Anatomie et de Cytologie Pathologiques, Hôpital Necker-Enfants Malades, Paris, France; (10) Services de Génétique et de Dermatologie, Hôpital Necker Enfants Malades, Paris, France ; (11) Université Paris V René Descartes, Paris, France

Contact : alain.hovnanian@inserm.fr

Les épidermolyses bulleuses simples (EBS) sont des maladies génétiques cutanées rares dues le plus souvent à des mutations dominantes des gènes codant les kératines 5 (KRT5) et 14 (KRT14). Elles sont responsables de décollements bulleux intra-épidermiques de sévérité variable. Elles sont divisées en trois groupes sur des critères cliniques et ultrastructuraux, avec une forte corrélation génotype-phénotype. La nouvelle nomenclature distingue l'EBS de Dowling Meara (EBS-DM) généralisée et sévère, l'EBS généralisée non Dowling Meara (EBS gen-nonDM) moins sévère et l'EBS localisée (EBS-loc) prédominant aux extrémités. Nous rapportons ici une étude génotype-phénotype rétrospective chez 31 familles atteintes d'EBS, d'origine caucasienne dans $74 \%$ des cas. 23 mutations distinctes ont été identifiées par séquençage : 7 mutations de KRT14 et 16 mutations de
KRT5 sont dominantes, une mutation de KRT14 est récessive. 9 de ces mutations sont nouvelles et suivent la corrélation génotype-phénotype décrite : 2 mutations faux sens de la région « Helix Termination Peptide » (HTP) du domaine bâtonnet conduisant au phénotype EBS-DM ; une délétion d'un acide aminé du domaine $\mathrm{H} 1$ de la tête de la molécule et cinq mutations faux sens des domaines $1 \mathrm{~A}$ et $2 \mathrm{~B}$ situées dans le domaine bâtonnet en dehors des régions « Helix Initiation Peptide " (HIP) et HTP, chez des patients EBS-loc. La mutation récessive est une mutation non sens de la KRT14 identifiée chez une patiente adoptée atteinte d'une forme généralisée (p.Q372X). Les 14 autres mutations ont été préalablement rapportées, parmi lesquelles 3 mutations récurrentes de KRT14. À noter que 5 de ces patients présentent un phénotype différent de celui initialement décrit dans la littérature : 3 patients EBS-loc portent des mutations préalablement rapportées chez de nombreux patients EBS-DM (mutations récurrentes p.R125C, p.R125H) ou chez des patients EBS gen-nonDM et EBS-loc (p.L325P). Deux autres patients EBS-DM dans notre étude portent des mutations préalablement rapportées chez des patients EBS gennonDM (p.L463P, p.E466D). Cette étude confirme l'existence de * points chauds " de mutations des KRT5 et KRT14 dans les domaines HIP ou HTP associé à l'EBS-DM. De façon surprenante, deux familles atteintes d'EBS-loc portent les mutations récurrentes p.R125H et p.R125C du domaine HIP de la KRT14, invariablement décrites chez de nombreux patients EBS-DM. L'observation de trois autres mutations associées à un phénotype différent de celui rapporté dans la littérature souligne la difficulté et l'importance d'évaluer le phénotype clinique des différentes formes d'EBS. La variabilité inter-indivuelle pour une même mutation confirme les récentes études sur l'influence de facteurs génétiques et/ou environnemeaux sur le sévérité des EBS. Ces observations sont les premières étapes vers l'identification de gènes modificateurs pouvant influencer l'expression clinique de ces formes d'EB.

Mots-clés : épidermolyse bulleuse simple, corrélation génotype-phénotype, génodermatoses.

IP499. CORRÉLATIONS GÉNOTYPE-PHÉNOTYPE DANS UNE COHORTE DE 30 PATIENTS ATTEINTS DE LA MALADIE DE DARIER : MUTATIONS FAUX-SENS VERSUS PTCS

M. Savignac (1), L. Lacaze-Buzy (1), C. Deraison (1), N. Antoni (2), J.M. Blancbonnet (3), J. Chevrant-Breton (4), P. Labrune (5), M.F. Jonkman (6), R. Tomb (7), Y. De Prost (8), A. Hovnanian (1, 9, 10) (1) INSERM, U563, Département de Génétique, Toulouse, France; (2) Service de Dermatologie, Hôpital Civil, Strasbourg, France; (3) Service de Dermatologie, CHU Dupuytren, Limoges, France ; (4) Service de Dermatologie, CHU Pontchaillou, Rennes, France; (5) Hôpital AntoineBéclère, Clamart, France; (6) Service de Dermatologie, Université Medical Center Groningen, Groningen, Pays Bas ; (7) Service de Dermatologie, Hôpital Hotel-Dieu, Beyrouth, Liban; (8) Service de Dermatologie, Hôpital Necker Enfants-Malades, Paris, France; (9) Services de Génétique et de Dermatologie, Hôpital Necker Enfants-Malades, Paris, France; (10) Université Paris V René Descartes, Paris, France

Contact : alain.hovnanian@inserm.fr

La maladie de Darier (MD) est une maladie génétique cutanée sévère rare, transmise sur le mode autosomique dominant. Elle se caractérise par des lésions hyperkératosiques prédominant dans les régions séborréhiques et les plis, d'étendue et de sévérité variable. Les lésions histologiques associent une acantholyse supra-basale et une dyskératose. Le gène de la MD, ATP2A 2 code pour l'isoforme 2 de la pompe Ca2+-ATPase (SERCA2) du reticulum endoplasmique. Les études fonctionnelles in vitro de ces mutations ont montré qu'elles conduisent à une perte du transport de $\mathrm{Ca} 2+$. ATP2 A2 est transcrit en trois isoformes SERCA2a, 2b et $2 c$ par épissage alternatif des trois derniers exons. $L$ 'isoforme $2 \mathrm{~b}$ est majoritaire dans l'épiderme. Nous avons étudié 11 cas familiaux et 19 cas sporadiques dont 2 présentent une forme segmentaire localisée de la MD. Nous avons identifié par séquençage du gène ATP2A2, 19 nouvelles mutations et 9 mutations précédemment rapportées. Les deux patients présentant une forme segmentaire portent une mutation d'ATP2A2 dans les kératinocytes issus des lésions cutanées, qui n'est pas retrouvée dans les leucocytes périphériques. Les patients ont été classés en 3 groupes de sévérité : bénigne (papules kératosiques éparses ou limitées à une ou deux régions) (4), modérée (papules plus étendues ou plaques verruqueuses localisées) (13) ou sévère (plaques verruqueuses coalescentes étendues) (7). Les 4 patients atteints d'une forme bénigne présentent tous des mutations non sens. Parmi les 13 patients atteints d'une forme modérée, 6 présentent des mutations conduisant à un codon stop prématuré (PTC), 4 portent des mutations fauxsens, 2 présentent une mutation d'épissage et un porte la délétion d'un acide aminé. Parmi les 7 patients atteints d'une forme sévère, 5 présentent une mutation faux-sens, 1 patient est porteur d'une mutation conduisant à 
un PTC et 1 patient présente une mutation d'épissage. Deux patients portent une mutation dans la région spécifique de SERCA2b, ce qui confirme qu'une mutation invalidant spécifiquement l'isoforme ATP2A2b est suffisante pour conduire au phénotype MD. Les patients de notre cohorte atteints d'une forme bénigne de la MD sont porteurs de mutations non sens et ceux présentant une forme sévère sont le plus souvent porteurs d'une mutation faux-sens $(5 / 7)$. Ceci confirme la notion selon laquelle les mutations faux-sens sont responsables de formes plus sévères de MD que les mutations conduisant à un PTC. Ceci est concordant avec les études fontionnelles qui ont montré que certaines mutations faux-sens peuvent bloquer l'activité de la pompe endogène et donc agir de façon dominante négative, ce qui expliquerait leur sévérité. À l'opposé, les mutations PTCs induisent une dégradation précoce de l'ARNm et rendent compte d'un mécanisme par haploinsuffisance. Cette étude conduit à envisager un mécanisme double, par haploinsuffisance (PTCs) ou par effect dominant négatif (faux-sens) selon la nature des mutations.

Mots-clés : maladie de Darier, calcium, ATP2A2.

\section{aP500. SYNDROME DE BIRT HOG DUBBE: PRÉSENCE DE GRANDS RÉARRANGEMENTS DE LA FOLLICULINE DANS 1/4 DES CAS}

N. Soufir (1), C. Kannengiesser (1), A. Bourillon (1), T. Frebourg (2), V. Descamps (3), E. Bourrat (4), B. Matard (5), B. Crestani (6), F. Grange (7), M. Rybojad (4), A. Taieb (8), A. Tazi (9), D. Lacombe (10), B. Grandchamp (1)

(1) Laboratoire de Génétique, Hopital Bichat, APHP, Universite Paris 7 , Paris, France. (2) Unité de Génétique Clinique, CHU Rouen, France. (3) Service de Dermatologie, Hopital Bichat, APHP, Universite Paris 7 , Paris, France ; (4) Service de Dermatologie, Hopital Saint Louis, APHP, Universite Paris 7, Paris, France ; (5) Service de Dermatologie, Hopital Tarnier, APHP, Universite Paris 5, Paris, France; (6) Service de Pneumologie, Hopital Bichat, APHP, Universite Paris 7, Paris, France; (7) Service de Dermatologie, CHU de Reims, Reims, France; (8) Service de Dermatologie, CHU de Bordeaux, Bordeaux, France: (9) Service de Pneumologie, Hopital Saint Louis, APHP, Universite Paris 7, Paris, France; (10) Service de Genetique, CHU Bordeaux, Bordeaux, France

Contact : nsoufir@yahoo.com

Introduction Le syndrome de Birt-Hogg-Dubé (BHD) est une génodermatose de transmission autosomique dominante caractérisée par des tumeurs folliculaire bénignes (fibrofolliculomes, FF), une atteinte pulmonaire (emphysème et/ou de pneumothorax spontané), un risque de cancer rénal souvent bilatéral, et un sur-risque possible de cancer colique. Le gène en cause est la folliculine (FLCN), situé sur le chromosome 17p11.2 De nombreuses mutations ont été identifiées, pour la grande majorité décalant le cadre de lecture, avec un point chaud dans l'exon 11. Patients et méthodes Soixante quatre prélèvements ont été adressés pour diagnostic moléculaire de syndrome de BHD. Après extraction de l'ADN, une exploration moléculaire du gène FLCN a été réalisée, par séquençage automatique (Applied Biosystems 3130 ) et recherche de grands réarrangements (MLPA et CGH array). Lorsqu'une mutation a été identifiée, elle a été systématiquement contrôlée sur un $2^{\mathrm{C}}$ prélèvement. La grande majorité des malades ont eu une consultation d'oncogénétique, une consultation dermatologique, une tomodensitométrie thoraco-abdominale. Résultats Des anomalies de la FLCN ont été identifiées chez 27 non apparentés $(42 \%)$. Le séquençage a permis de détecter 15 anomalies différentes, non précédemment décrites, chez 20 probands. Il s' agissant principalement de mutations frameshift $(50 \%)$, de mutations non sens $(25 \%)$, de mutations d'épissage ( $10 \%)$, et de petites délétions en phase $(10 \%)$. Trois mutations étaient retrouvées plus d'une fois. De manière intéressante, et pour la première fois, des grands réarrangements du gène ont été identifiés par MLPA (puis confirmées par CGH array) chez 7 malades ( $25 \%$ des mutations de la FLCN) : Il s'agissait de délétions intragéniques dans 6 cas (délétion de l'exon 1,2 malades; délétion des exons 1 à $4: 2$ malades; délétion des exons 2 à $6: 1$ malade; délétion de l'exon 12 : 1 malade et d'une duplication (exons $2,4,8$ ) dans un cas. Les FFs était fortement associés aux mutations de la FLCN $(92 \%)$. Une atteinte respiratoire (pneumothorax et/ou emphysème) était présente chez $67 \%$ des sujets mutés. Un seul malade muté était porteur d'un cancer du rein (bilatéral), mais un cancer du rein était retrouvé dans $18 \%$ des familles mutées. Un cancer du colon chez un apparenté du premier degré était présent dans $29 \%$ des familles pour lesquelles cette information était disponible. II existait, pour une mutation identique retrouvée dans 3 familles différentes, une variation phénotypique inter et intrafamiliale très nette. Conclusion: Notre étude montre pour la première fois l'existence de réarrangements de grande taille dans une proportion importante de malade atteints de BHD $(25 \%)$, soulignant l'importance de leur recherche lors du diagnostic moléculaire. La fré- charge multidisciplinaire avec surveillance régulière, et un diagnostic présymptomatique chez les apparentés.

Mots-clés : folliculine, mutations, délétions.

P501. IDENTIFICATION D'UNE MÊME MUTATION CHEZ DES ENFANTS ATTEINTS DE DEUX FORMES DIFFÉRENTES D'ÉPIDERMOLYSE BULLEUSE DYSTROPHIQUE

H. Ouragini (1), F. Cherif (2), G. Floriddia (3), M. Pascucci (3), A. Ben Osman-Dhahri (2), D. Castiglia (3), S. Abdelhak (1)

(1) UR: Exploration moléculaire des Maladies Orphelines d'Origine Génétique, Institut Pasteur de Tunis, Tunisie; (2) Service de Dermatologie, Hôpital La Rabta de Tunis, Tunisie; (3) Laboratorio di Biologia Molecolare e Cellulare, Istituto Dermopatico dell'Immacolata-IRCCS, Roma, Italy

Contact : ohouyem@gmail.com

L'épidermolyse bulleuse dystrophique (EBD) est caractérisée par un clivage sous-épidermique entraînant un décollement cutané. Selon le mode de transmission et les manifestations cliniques, l'EBD est différencié en deux groupes : un dominant (EBDD) et un récessif (EBDR), qui inclut 2 sous-types majeurs l'EBDR généralisée sévère (EBDRgs), et les autres formes d'EBDR généralisées (EBDRg). Toutes les formes d'EBD sont dues à des mutations au niveau du gène COL7Al, qui code pour le collagène VII. Au cours de notre analyse mutationnelle des patients tunisiens atteints d'EBD, nous avons identifié deux mutations, p.R1763X et c. $7344 \mathrm{G}>\mathrm{A}$, présents aussi bien chez des individus atteints de la forme sévère EBDRgs que chez des individus atteints de la forme non sévère EBDRg. L'hétérogénéité clinique pour une même mutation est très fréquente au niveau de cette pathologie, mais seule une étude a rapporté des cas avec une même mutation présentant deux formes d'EBD de sévérité différente. Il a été démontré que cette variabilité était due à l'influence d'un polymorphisme au niveau du gène codant pour la métalloprotéinase 1 , le gène MMP1 (Titeux et al., 2008). Cependant, notre étude n'a pas montré de corrélation entre ce polymorphisme et la sévérité de la maladie chez nos patients, suggérant l'influence d'autres facteurs génétiques influençant cette variabilité.

Mots-clés : épidermolyse bulleuse dystrophique, mutation, hétérogénéité phénotypique.

IP502. IDENTIFICATION DE MUTATIONS DANS LE GÈNE WNTIOA DANS UNE COHORTE DE PATIENTS PRÉSENTANT DIVERSES FORMES DE DYSPLASIES ECTODERMIQUES

C. Cluzeau (1), P. Guigue (1), H. Kayserili (2), S. Mansour (1), M. Le Merrer (3), N. Chassaing (4), P. Calvas (4), A. Munnich (1), C. Bodemer (1, 5), S. Hadj-Rabia (1, 5), A. Smahi (1)

(1) Université Paris Descartes, et INSERM U781, Hôpital Necker-Enfants Malades, Paris 75015, France; (2) Department of Medical Genetics, Istanbul Medical Faculty, Istanbul University, Turquie: (3) Service de génétique, Hôpital Necker-Enfants Malades, Paris 75015, France ; (4) Service de Génétique médicale, Hôpital Purpan, Toulouse, France ; (5) Service de dermatologie, Hôpital Necker-Enfants Malades, Paris 75015 , France

Contact : celine.cluzeau@inserm.fr

Les dysplasies ectodermiques (ou ED) forment un groupe hétérogène de 170 syndromes rares caractérisés par l'atteinte des annexes épidermiques (poils, dents, ongles, glandes sudoripares). Une cinquantaine de ces syndromes ont une origine génétique connue. La dysplasie ectodermique anhidrotique ou hypohidrotique (ou HED) est la forme la plus fréquente des ED. Trois gènes sont responsables des HED : EDA (codant l'ectodysplasine), EDAR et EDARADD. Différents modèles murins ont permis de montrer que la voie de signalisation Wnt/beta-caténine est essentielle au développement des annexes épidermiques. Une première mutation a été identifiée dans le gène WNT10A chez des patients Libanais présentant une dysplasie ectodermique rare, la dysplasie odonto-onycho-dermique (OODD), avec une variabilité clinique intrafamiliale assez importante. WNT10A et WNT10B sont co-exprimés dans les placodes des annexes épidermiques en développement, et leurs séquences protéiques sont fortement identiques. Nous avons donc émis l'hypothèse que ces deux gènes pourraient être responsables de dysplasies ectodermiques variées. Au cours de notre étude, deux nouveaux articles ont été publiés rapportant des mutations de WNT10A, dont l'un confirmant l'hypothèse de l'implication de ce gène dans des dysplasies ectodermiques au sens large. Nous avons réuni une cohorte de 99 patients d'origine Française et Turque, présentant diverses formes familiales ou sporadiques de dysplasies ectodermiques. $80 \%$ des patients de la cohorte présente une HED, les gènes EDA, EDAR et EDARADD étant exclus chez ces patients. Les autres patients de la 
cohorte présentent des formes atypiques de dysplasies ectodermiques, tous ayant au minimum des atteintes dentaires sévères et certains présentant également des atteintes cutanées ou extra-cutanées. Nous avons identifié sept mutations au total dans le gène WNT10A, dont cinq nouvelles. $\mathrm{La}$ séquence du gène WNT10B est par contre totalement normale. Parmi ces mutations de WNT10A, nous avons identifié pour la première fois une duplication de sept nucléotides au sein du premier exon du gène, qui conduit à un décalage du cadre de lecture et un codon stop prématuré. Une mutation de l'exon 3 (c.682T $>A$, p.Phe228Пle) est très fréquemment retrouvée dans notre cohorte (11 patients hétérozygotes, 3 patients homozygotes). Tous les patients pour lesquels un changement a été identifié dans ce gène ont une atteinte dentaire sévère, avec quelques autres signes cliniques sur les annexes épidermiques ou étrangers aux annexes. La majorité des patients sont hétérozygotes composites ou homozygotes. Nous poursuivons l'étude moléculaire pour les patients n'ayant qu'une seule mutation identifiée, afin de déterminer si certaines mutations pourraient être transmises sur un mode dominant ou si des délétions partielles du gène ou des mutations d'épissage au sein du promoteur du gène pourraient être présentes sur le second allèle de ces patients.

Mots-clés : dysplasie ectodermique, WNT10A.

P503. ANALYSE CLINIQUE ET MOLÉCULAIRE SUR 166 PATIENTS ATTEINTS DE SCLÉROSE TUBÉREUSE DE BOURNEVILLE

V. Hebepin-Granados, C. Keintz, A. Combes, M. Deville, M.F. Gilet, C. Siterre, R.L. Touraine

Service de Génétique Clinique, Chromosomique et Moléculaire, $\mathrm{CHU}$ Nord, Saint Étienne, France

Contact : viviana.herbepin@chu-st-etienne.fr

La Sclérose Tubéreuse (STB) est une maladie autosomique dominante caractérisée principalement par le développement de multiples harmatomes. Elle est causée par des mutations affectant les gènes suppresseurs de tumeurs TSC1 et TSC2. De très nombreuses mutations ont été décrites, la plupart dans une ou quelques familles. Nous présentons les résultats de 166 patients qui ont été étudiés dans notre laboratoire. Les recherches de mutation ont été effectuées par DHPLC/ séquençage des régions codants dans les deux gènes, et par MLPA pour les délétions de grande taille. Aucune mutation n'a été identifiée dans $15 \%$ des cas (25 patients). Différentes mutations pathogènes ont été identifiées chez 141 patients $(85 \%)$. Pour 7 patients $(4,2 \%)$, une grande délétion a été trouvée ( 3 sur TSC1 et 4 sur TSC2). Chez 49 patients ( $30 \%$ des mutations ponctuelles identifiées) nous avons identifié des mutations non décrites. Elles comprenaient 19 petites mutations sur TSC 1 ( 11 cas familiaux, 6 cas sporadiques et 2 cas non déterminé) et 30 impliquent TSC2 ( 5 cas familiaux, 19 cas sporadiques et 6 cas non déterminé). Dans certaines familles les symptômes peuvent être extrêmement modérés, ce qui peut compliquer l'interprétation des résultats lorsque la mutation potentielle est un faux-sens non décrit. Toutefois, aucun cas de non pénétrance n'a été décrit. En l'absence de mutation identifiée, dans certains cas le diagnostic doit être remis en cause. Il n'y a toutefois pas de corrélation génotype phénotype, en dehors d'une plus fréquente sévérité des mutations de TSC2. Ainsi, l'étude moléculaire dans la STB a sa place avec l'expertise clinique.

Mots-clés : sclérose tubéreuse, mutations, TSC1 et TSC2.

IP504. CORRÉLATION GÉNOTYPE-PHÉNOTYPE CHEZ LES PATIENTS NF1 MICRODÉLÉTÉS

E. Pasmant $(1,2)$, A. Sabbagh $(1,2)$, B. Parfait $(1,2)$, M.J. Hamel (2), P. Wolkenstein (3), M. Vidaud (1, 2), M. Upadhyaya (4), D. Vidaud $(1,2)$ (1) UMR745 INSERM, Faculté des Sciences Pharmaceutiques et Biologiques Paris-Descartes, Paris, France ; (2) Service de Biochimie et de Génétique Moléculaire, Hôpital Beaujon, AP-HP, Clichy, France ; (3) Service de Dermatologie, Hôpital Henri Mondor, AP-HP, Créteil, France ; (4) Institute of Medical Genetics, Cardiff University, Cardiff, United Kingdom Contact : eric.pasmant@gmail.com

Introduction : La neurofibromatose de type 1 (NF1, MIM162200) est une maladie héréditaire monogénique dont l'expression phénotypique, tant à l'échelle intrafamiliale qu' inter-familiale, est très variable. Cette importante variabilité d'expression pourrait s'expliquer en partie par l'extrême hétérogénéité des mutations délétères du gène NF1 (localisé en 17q11.2). Mais à ce jour, peu de corrélations génotype-phénotype ont été mises en évidence. Il semblerait toutefois que des délétions entières du gène NF1 (retrouvées dans 5 à $10 \%$ des NF1) incluant une série de gènes situés dans les régions environnantes, soient plus souvent associées à un phénotype particulier : le «syndrome de microdélétion NF1 ", caractérisé par une forme plus sévère de la maladie. Il existe en effet des microdélétions récurrentes du gène NF1 : de type 1 (majoritaires, d'une taille de $1.4 \mathrm{Mb}$ environ) et de type $2(1,2 \mathrm{Mb})$ qui impliquent des séquences répétées situées de part et d'autre du locus NF1: les NF1-REP-A et NF1-REP-C pour les microdélétions de type 1 et le gène SUZ12 (suppressor of zeste 12 homolog) et son pseudogène SUZ12P pour les type 2. Des délétions atypiques (non récurrentes) ont également été décrites. Objectif: L'objectif de cette étude a été d'évaluer si un phénotype clinique particulier est associé aux microdélétions du gène NF1, en comparant l'incidence de 13 signes cliniques entre 85 patients NF1 microdélétés et des patients NF1 avec une mutation intragénique, parfaitement appariés pour l'âge et le sexe. Afin de réaliser cette étude nécessitant une parfaite caractérisation de ces délétions au niveau moléculaire, nous avons développé une puce dédiée de haute résolution (comparative genomic hybridization array) de format Agilent ${ }^{*} 8 \times 15 \mathrm{~K}$. La puce inclut un total de 14207 sondes oligonucléotidiques $(60$ mers $)$ réparties sur l'ensemble du chromosome 17 dont 12314 sondes sur un intervalle de $8 \mathrm{Mb}$ incluant le locus NF1 $(\sim 300 \mathrm{~kb})$ et l'ensemble des délétions décrites dans la littérature. Résultats-Discussion: Contrairement aux précédentes méthodes, notre puce dédiée a permis de différencier sans ambiguïté les trois types de microdélétions (type 1 , type 2 et atypique) et de cloner les points de cassure des microdélétions atypiques. Les résultats de l'étude de corrélation génotype-phénotype montrent une association significative de certains traits cliniques - difficultés d'apprentissage, dysmorphie faciale, présence de neurofibromes souscutanés, risque plus élevé de tumeurs - avec les microdélétions du gène NF1. Cette approche permettra d'identifier des gènes candidats qui sont directement impliqués dans les différentes manifestations cliniques caractéristiques du syndrome de microdélétion NF1.

Mots-clés : neurofibromatose de type 1, NF1, microdeletion.

P505. MISE AU POINT DU DOSAGE GÉNIQUE DU GÈNE OCRLI PAR MULTIPLEX LIGATION-DÉPENDENT PROBE AMPLIFICATION (MLPA). APPLICATION AU SYNDROME DE LOWE ET À LA MALADIE DE DENT

C. Coutton, N. Monnier, J. Rendu, J. Lunardi

Laboratoire de Biochimie et Génétique Moléculaire de l'ADN, CHU A. Michallon, Grenoble, France

Contact : CCoutton@chu-grenoble.fr

Le syndrome oculo-cérébro-rénal de Lowe et la maladie de Dent sont deux maladies génétiques très rares de transmission récessive liée au chromosome $\mathrm{X}$ chez lesquelles des délétions du gène OCRL1 ont été décrites. Bien que simple à détecter chez un homme, ces réarrangements sont délicats à mettre en évidence chez les femmes potentiellement conductrices de la maladie avec les techniques de diagnostic de première intention (ex : séquençage) en raison de la présence de 2 copies du gène OCRL1. Leur détection, de même que la détection d'autres réarrangements génomiques potentiellement causals de la maladie tels que les duplications ou les délétions en mosaïque, imposent l'utilisation de techniques quantitatives complémentaires souvent longues, fastidieuses et peu résolutives. La technique de Multiplex Ligation-dependent Probe Amplification (MLPA) se distingue des autres techniques quantitatives par sa rapidité et son excellente résolution. Nous avons modélisé 24 sondes MLPA spécifiques des 24 exons du gène OCRL1 selon un algorithme propre à notre laboratoire. Les sondes ont ensuite été validées sur un panel de patients sains et atteints. Cette technique a permis de préciser le statut de conductrice de 5 femmes avec un apparenté atteint porteur d'une délétion génomique d'OCRL1. Nous avons également montré que les sondes utilisées permettaient de détecter un taux de mosaïcisme d'au moins $20 \%$. En dépit de sa sensibilité, aucun réarrangement en mosaïque ou duplication n'a cependant été retrouvé chez 15 patients présentant un tableau clinique évocateur du syndrome de Lowe et chez lesquels l'analyse préalable par séquençage d'OCRL1 s'était révélée non contributive. Notre technique MLPA est parfaitement spécifique du gène OCRL1, sensible mais aussi robuste et rapide. Elle est ainsi immédiatement applicable en routine comme technique de choix pour déterminer le statut des femmes potentiellement conductrices de délétions. C'est également un outil de diagnostic complémentaire du séquençage pour le syndrome de Lowe et la maladie de Dent. Notre étude corrobore les précédents résultats publiés dans la littérature indiquant que les duplications du gène OCRL1 sont des événements probablement exceptionnels. Ils remettent aussi en question l'hypothèse que les réarrangements génomiques en mosaïque contribueraient à expliquer la fraction de patients avec un phénotype incomplet ou intermédiaire du syndrome de Lowe.

Mots-clés : syndrome oculo-cérébro-rénal de Lowe, OCRL1, MLPA. 
P506. SPECTRE DES MUTATIONS DU GÈNE COLAA5 ET PRÉSENTATION CLINIQUE DES PATIENTS AVEC SYNDROME D'ALPORT LIÉ À L'X

G. Benoit (1, 2), E. Machuca (1, 3), A. Pawtowski (4), V. Morinière (4), L. Heidet $(1,5,6)$, C. Antignac $(1,4,6)$

(1) Inserm, U574, Hôpital Necker-Enfants Malades, Paris, France ; (2) CHU Sainte-Justine, Université de Montréal, Canada ; (3) Pontificia Universidad Católica de Chile, Escuela de Medicina, Chile; (4) Assistance Publique-Hôpitaux de Paris (AP-HP), Département de Génétique, Hôpital Necker-Enfants Malades, Paris, France ; (5) Assistance Publique-Hôpitaux de Paris (AP-HP), Centre de Référence des Maladies Rénales Héréditaires de l'Enfant et de l'Adulte (MARHEA), Service de Néphrologie Pédiatrique, Hôpital Necker-Enfants Malades, Paris, France ; (6) Université Paris Descartes, Faculté de Médecine Paris Descartes, Paris, France Contact : corinne.antignac@inserm.fr

Le syndrome d'Alport (SA) est une affection héréditaire caractérisée par une néphropathie hématurique progressive souvent associée à une surdité de perception. Des mutations dans le gène COL4A5 sont responsables de la forme liée à l'X de la maladie et des anomalies de distribution de la chaîne alpha5 du collagène de type IV peuvent être documentées à la biopsie rénale ou cutanée. L'objectif de notre étude est de déterminer le spectre des mutations COLAA5 et le phénotype associé à partir d'une cohorte de 68 individus non apparentés (sexe masculin $(\mathrm{m}): 48$, sexe féminin $(\mathrm{f}): 20$ ), ceci représentant notre expérience des 3 dernières années dans la détection de mutations COL4A5. La détection de mutations dans tous les exons codants du gène a été effectuée par séquençage direct. Des mutations ont été identifiées dans 57 familles, le taux de détection atteignant $84 \%$ (m: $90 \%$, f : $70 \%)$. Quarante-trois sont de nouvelles mutations. La plupart des mutations $(72 \%)$ sont des substitutions. Vingt-six des 28 mutations faux-sens affectent un codon glycine. Dix petites $(1-4 \mathrm{pb})$ délétions, une délétion de 9 paires de base, une petite insertion, 2 petites duplications, une duplication de 18 paires de base et un réarrangement complexe ont également été détectés. L'âge médian au moment du diagnostic génétique moléculaire est de 33 ans ( $\mathrm{m}: 33$, f : 32) (3-75 ans). Vingt-cinq patients $(\mathrm{m}: 22, \mathrm{f}: 3)$ ont atteint l'insuffisance rénale terminale (IRT) à un âge médian de 28.5 ans ( $\mathrm{m}: 29$ ans, $\mathrm{f}: 27$ ans). La majorité des femmes ont une évolution rénale bénigne; trois (mutations p.G641V, p.G1131fs1151X et p.P1292fs1304X) ont toutefois atteint l'IRT entre 27 et 31 ans. Un déficit auditif est présent chez 27 individus ( $\mathrm{m}: 26, \mathrm{f}: 1$ ), Lorsque documentée, l'expression de la chaîne alpha5 (IV) sur la biopsie cutanée est anormale pour $33 \%$ des individus de sexe masculin et $88 \%$ des individus de sexe féminin. En conclusion, le SA lié à l'X est une maladie hétérogène, notamment en regard de l'âge au moment de l'IRT. Bien qu'une anomalie de l'expression de la chaîne alpha5 (IV) sur la biopsie de peau puisse confirmer la présence de SA lié a l'X, des biopsies cutanées normales sont retrouvées chez un nombre significatif de patients avec mutations COL4A5 documentées. Étant donné le taux élevé de détection par séquençage direct, la recherche de mutation COL4A5 revêt une importance clinique certaine, notamment pour le conseil génétique et le diagnostic prénatal.

Mots-clés : Alport, COL4A5, mutations.

EP507. DYSGÉNÉSIE TUBULAIRE RÉNALE AUTOSOMIQUE RÉCESSIVE. NOUVELLES MUTATIONS DANS LES GÈNES DU SYSTÈME RÉNINE-ANGIOTENSINE (SRA)

O. Gribouval, V. Morinière, A. Pawtowski, C. Antignac, M.C. Gubler Inserm U574, Université Paris Descartes, Département de Génétique, AP-HP, Hôpital Necker Paris, France

Contact : olivier.gribouval@inserm.fr

La dysgénésie tubulaire rénale (DTR) autosomique récessive est une néphropathie sévère du fœtus se manifestant par une anurie précoce et persistante responsable d'oligoamnios, de la séquence de Potter et, le plus souvent, de mort périnatale. Une hypotension réfractaire et un retard d'ossification de la voute crânienne sont observés chez les nouveau-nés survivants. Les tubes proximaux sont absents ou en nombre très réduit. II s'y associe constamment à une anomalie majeure de l'expression rénale de rénine. Nous avons montré que cette néphropathie était liée à des mutations des gènes codant les différentes protéines du SRA. Depuis, l'analyse moléculaire des gènes du SRA a été réalisée chez 57 nouveaux patients appartenant à 37 familles. Des mutations homozygotes (18) ou hétérozygotes composites (11) ont été identifiées dans 29 familles tandis qu'une seule mutation du gène ACE a été détectée dans 4 familles. La plupart des mutations (24/33) touchaient le gène $\mathrm{ACE}$ tandis que des mutations dans les gènes REN, AGT et AGTR 1 étaient observées dans respectivement 5,2 et 2 familles. Ces mutations mutations faux sens (13/37), délétion d'un acide aminé (1/37) et mutations modifiant le peptide signal de l'enzyme de conversion (5/37). Sept mutations ont été observées dans 2 familles non apparentées. Six enfants (mutation de REN ( 1 cas) et du gène ACE ( 5 cas)) ont survécu, pour 5 d'entre eux après plusieurs jours ou mois de dialyse péritonéale. Trois d'entre eux ont été transplantés à respectivement 3,4 et 5 ans, deux patients ont une insuffisance rénale chronique à respectivement 3 et 7 ans, le dernier a une fonction rénale normale à 7 ans. Aucune corrélation génotype-phénotype n'a pu être établie : le phénotype clinique et morphologique est le même quel que soit le gène muté ; dans la même famille des formes de sévérité différente (mort in utero d'un fœtus survie d'un enfant) ont été observées. Aucune mutation n'a été identifiée chez les fœtus appartenant à 4 familles. La présence de symptômes inhabituels (anasarque, retard de croissance intra-utérine) dans 2 d'entre elles suggérait que la DTR était secondaire à une pathologie non identifiée. Dans une troisième famille, consanguine, l'expression rénale normale de rénine éliminait l'implication du SRA, ce qu'ont confirmé les études de liaison. En conclusion, cette étude confirme le rôle majeur et probablement exclusif des mutations des gènes du SRA dans la survenue de la forme autosomique récessive de DTR. Le diagnostic de la maladie basé sur un ensemble de critères cliniques, morphologiques et immunohistologiques doit conduire, après exclusion des DTR secondaires (cardiopathies, exposition aux drogues bloquant le SRA...) à l'analyse moléculaire des gènes du SRA permettant, par l'identification du défaut génétique, de proposer un conseil génétique et un diagnostic prénatal précoce.

Mots-clés : mutations, système rénine-angiotensine, dysgénésie tubulaire rénale.

\section{ШP508. INTÉRÊTS ET LIMITES DU DIAGNOSTIC MOLÉCU- LAIRE DE LA POLYKYSTOSE RÉNALE AUTOSOMIQUE DOMINANTE}

M.P. Audrezet (1), E. Cornec-Le Gall (2), I. Quere (1), J. Creff (1), B. Mercier (1), Y. Le Meur (2), C. Ferec (1)

(1) Laboratoire de Génétique Moléculaire, CHU de Brest, France; (2) Service de Néphrologie, CHU de Brest, France

Contact : marie-pierre.audrezet@chu-brest.fr

La Polykystose rénale autosomique dominante est la maladie génétique de transmission monogénique la plus fréquente, puisqu'elle touche 1 personne sur mille, environ 12,5 millions d'individus dans le monde et est responsable de $7 \%$ des causes d'insuffisance rénale terminale chez l'adulte. Deux gènes responsables de la polykystose rénale autosomique dominante ont été caractérisés. Le gène PKD1, responsable de $85 \%$ des cas est localisé sur le chromosome 16 et code pour la polycystine 1 . Le gène $\mathrm{PKD} 2$, responsable de $15 \%$ des cas, porté par le chromosome 4 , code pour la polycystine 2 . L'étude moléculaire de ces gènes est extrêmement délicate, particulièrement pour PKD1 qui compte 46 exons, est très riche en nucléotides $\mathrm{G}$ et $\mathrm{C}$, présente sur les trois quarts de sa séquence, $98 \%$ d'homologie avec 6 pseudogènes. Plus de 300 mutations ont été décrites dans le gène PKD1 et près de 100 dans le gène PKD2, la majorité d'entre elles étant des mutations privées. L'analyse moléculaire de ces deux gènes n'est pas à l'heure actuelle proposée à titre systématique, le diagnostic de la polykystose rénale autosomique dominante étant surtout un diagnostic clinique qui repose sur les critères échographiques de Ravine. Il peut cependant s'avérer contributif dans certains cas de formes atypiques, en l'absence d'antécédents familiaux due à la survenue d'une mutation de novo, ou encore pour la sélection de donneurs indemnes dans les familles en vue d'une greffe... C'est pourquoi nous avons développé une stratégie d'analyse par séquençage des gènes PKD1 et PKD2, ainsi que la recherche des grands réarrangements par PCR quantitative. 17 amplifications dont 3 PCR multiplexes, et 76 réactions de séquence permettent d'analyser la totalité de la séquence codante ainsi que les jonctions intron-exons de ces deux gènes. Ces conditions d'étude ont été appliquées à l'étude de notre cohorte de patients européens et nous ont permis d'identifier 120 mutations différentes dans le gène PKD1 et 12 mutations différentes dans le gène PKD2, avec un taux de couverture des mutations supérieur à $80 \%$. Ces anomalies se répartissent en 53 mutations entraînant un décalage du cadre de lecture, 47 mutations non-sens, 13 mutations d'épissage, 9 mutations faux-sens, 8 courtes insertions ou délétions sans décalage du cadre de lecture, et 1 délétion complète du gène. 92 d'entre elles ont été identifiée chez nos patients pour la première fois. Cette étude est une première étape vers la mise en évidence de corrélations génotype-phénotype, qui, à l'avènement des thérapies ciblées actuellement à l'essai, pourraient se révéler utiles pour le néphrologue.

Mots-clés : polykystose renale, PKD 


\section{IP509. RÉLATION GÉNOTYPE-PHÉNOTYPE DANS UNE COHORTE DE 100 PATIENTS AVEC ACIDOSE TUBULAIRE RÉNALE DISTALE (ATRD)}

R. Vargas-Poussou (1), A. Blanchard (2), D. Kahila (1), N. Le Pottier (1), I. Roncelin (1), A. Venisse (1), P. Houillier (3), X. Jeunemaitre (1) et le Réseau Français pour l'étude des tubulopathies

(1) Département de Génétique, AP-HP Hôpital Européen Georges-Pompidou, Paris, France; (2) Centre d'Investigations Cliniques, AP-HP Hôpital Européen Georges-Pompidou, Paris, France ; (3) Service d'Exploration Fonctionnelles rénales et métaboliques, AP-HP Hôpital Européen Georges-Pompidou, Paris, France

Contact : rosa.vargas@egp.aphp.fr

Les ATRd sont des maladies rares dues à un défaut d'excrétion de $\mathrm{H}+$ par les cellules intercalaires du tube collecteur. Les formes récessives se manifestent dans la $1^{\text {ro }}$ année de vie (déshydratation, retard de croissance, acidose hyperchlorémique, hypokaliémie néphrocalcinose et parfois surdité). Les formes dominantes chez l'adolescent ou l'adulte (lithiase, ostéoporose, acidose modérée parfois révélait après charge acide, ATRd incomplète). Les formes récessives sont dues à des mutations des gènes des sous-unités B1 et a4 de la H+ATPase (ATP6V1B1 et ATP6V0A4) et les dominantes à des mutations du gène de l'échangeur $\mathrm{Cl} / \mathrm{HCO} 3$ (SLC4A1). Dans le sud-est asiatique, des formes récessives associant ovalocytose et ATRd ont été décrites. Nous avons analysé 100 patients avec ATRd : récessive $n=79$, dominante $\mathrm{n}=21$ ( 10 cas d'ATRd incomplète). Les données au diagnostic ont été recueillies à partir d'un questionnaire standardisé. Dans les familles consanguines, le gène a été ciblé par l'analyse des haplotypes. Les 3 gènes ont été analysés par séquençage direct. Dans le groupe à transmission récessive : détection de 14 mutations de ATP6V1B Idans 34 familles et de 23 mutations de ATP6V0A4 dans 30 familles (taux de détection de $81 \%$ ). Absence de liaison aux 2 locus observée pour 2 familles. Phénotype au diagnostic des patients avec mutations ATP6V1B1 vs mutations ATP6V0A4 : âge au diagnostic $0.4[0,25-1.08]$ vs $0.08[0,04-0,28]$ ans $(\mathrm{p}=0,0003)$, $\mathrm{pH}$ Sang $7.28[7,19-7,31]$ vs $7,15[7,10-7,21](\mathrm{p}=0,004)$, $\mathrm{CO} 2 \mathrm{t} 13,4[12-16]$ vs $11[8-14] \mathrm{mmol} / \mathrm{l}(\mathrm{p}=0,02), \mathrm{K}+2,8[2,6-3,5]$ vs 3,05 $[2,6-3,47]$ (ns) et $\mathrm{pH}$ Ur en acidose $7,2[7,0-7,6]$ vs $7,5[7,0-8,0]$ (ns). La surdité est plus fréquemment associée aux mutations ATP6V1B1, 20/34 vs 13/30 pour ATP6V0A4. La sévérité de la surdité est variable dans les 2 groupes ainsi que l'âge au diagnostic de celle-ci ( 2 mois à 10 ans pour ATP6V0A4 et 5 mois à 19 ans pour ATP6V1B1). Dans le groupe à transmission dominante : détection de 8 mutations de SLC4A1dans 10 familles avec ATRd complète et aucune dans les 10 familles avec ATRd incomplète. Un patient originaire de Madagascar présentant ATRd + ovalocytose est porteur de 2 mutations. Les données pour ce groupe sont : âge au diagnostic 16,5 [15-31] ans, pH Sang 7,32 [7,25-7,40], CO2t $19[16,15-19,85]$ $\mathrm{mmol} / \mathrm{l}, \mathrm{K}+3,15[2,97-3,32]$ et $\mathrm{pH}$ Ur en acidose $6,8[6,7-7,0]$. Ces données sont significativement différentes des celles des patients avec ATRd récessive à l'exception de la kaliémie. Une confirmation génétique a été effectuée pour $83 \%$ de patients avec ATRd. Dans les formes récessives, le groupe ATP6V0A4 a un diagnostic plus précoce et une acidose plus sévère. La surdité (sévérité et âge au diagnostic) est variable, indépendamment du gène impliqué. L'ATRd dominante représente $13,5 \%$ des cas dans cette cohorte. Aucune mutation n'a été détectée chez le groupe ATRd incomplète. Ces données sont en faveur d'une hétérogénéité génétique. Un arbre décisionnel est proposé.

\section{aP510. LE SYNDROME NÉPHROTIQUE CONGÉNITAL DANS LES POPULATIONS NON-FINLANDAISES}

E. Machuca $(1,2)$, F. Nevo (1), G. Benoit $(1,3)$ O. Gribouval (1), A. Pawtowski (1), C. Antignac $(1,4,5)$

(1) Inserm U574, Hôpital Necker-Enfants Malades, Paris, France; (2) Escuela de Medicina, Pontificia Universidad Católica, Santiago, Chile: (3) CHU Sainte-Justine, Université de Montréal, Canada ; (4) Faculté de Médecine Paris Descartes, Université Paris Descartes, Paris, France ; (5) Département de Génétique, Hôpital Necker-Enfants Malades, Paris, France

Contact : eduardo.machuca@inserm.fr

Le syndrome néphrotique congénital (SNC) est une maladie autosomique récessive caractérisée par une protéinurie massive, un gros placenta et des oedèmes importants se manifestant dans les trois premiers mois de vie. Des mutations dans le gène NPHS1, codant la néphrine, sont la cause principale des SNC dans la population finlandaise. Des mutations dans les gènes NPHS2, WT1, LAMB2 et PLCE1 (codant respectivement la podocine, le Wilm's tumor 1, la laminin beta2 et la phospholipase C epsilon 1) ont aussi été identifiés dans les SNC. Le but de notre étude est de caractériser la présentation clinique, les trouvailles histologiques et les corrélations génotype-phénotype dans une large cohorte non finlandaise de patients avec SNC. Les échantillons d'ADN et les données cliniques de 117 patients (107 familles) présentant un SNC ont été collectés. Le séquençage direct du gène NPHS1 a été effectué ; en l'absence de mutations NPHS1, la recherche de mutations NPHS2 a aussi été effectuée. Des mutations dans les gènes WT1, LAMB2 et PLCE1 ont été recherchées dans les cas avec ambiguitté sexuelle, anomalies oculaires ou sclérose mésangiale diffuse (SMD). Des mutations pathogènes homozygotes ou hétérozygotes composites de NPHS1 ont été identifiées chez 63 cas ( 60 familles). Dans 5 cas ( 4 familles), un seul allèle muté a été détecté. Vingt-six sont de nouvelles mutations, dont une délétion homozygote des exons 12 à 22 . Des mutations dans le gène NPHS2 ont été identifiées chez 20 patients ( 16 familles). Des mutations de LAMB2 et WT1 ont été détectées chez 3 cas de syndrome de Pierson et 2 autres cas de SMD, respectivement. Une mutation hétérozygote de PLCE1 a été détectée dans un cas. Un début de la maladie dans la première semaine de vie était plus fréquent chez les patients avec mutations NPHS1 que NPHS2 (50/68 vs $13 / 20$ cas ; $\mathrm{P}=0,002$ ) (début de la maladie : médiane 2 vs 29 jours ; $\mathrm{P}=0,003$ ). Le poids à la naissance et l'incidence de prématurité des patients avec mutations NPHS1, NPHS2 ou sans mutation étaient similaires. Une histologie rénale compatible avec celle retrouvée dans les cas de SNC de type finlandais a été observée chez $35 / 40$ cas avec mutations NPHS1, alors que la majorité des patients avec mutations NPHS2 ont présenté des lésions glomérulaires minimes ou d'hyalinose segmentaire et focale. Les cas avec mutations NPHS1 ont atteint l'insuffisance rénale terminale significativement plus tôt que ceux avec mutations NPHS2 (temps médian de survie rénale 3,3 vs 6.6 ans ; $\mathrm{P}=0,015$ ). En conclusion, dans les populations non finlandaises, les mutations NPHS1 sont la cause principale des SNC se présentant dans la première semaine de vie alors que des mutations NPHS2 sont fréquemment identifiées dans les cas avec syndrome néphrotique débutant après le premier mois de vie. Comparativement aux patients avec mutations NPHS1, les cas avec mutations NPHS2 présentent une histologie différente et une survie rénale significativement meilleure.

Mots-clés : syndrome néphrotique congénital, néphrine, podocine.

\section{IP511. SYNDROME DE GITELMAN : ANALYSE D'UNE FAMILLE} AVEC TRANSMISSION APPAREMMENT DOMINANTE

R. Vargas-Poussou (1), R. De La Faille (2), A. Venisse (1), V. Nau (1), P. Houillier (2), X. Jeunemaitre (1)

(I) Département de Génétique, AP-HP Hôpital Européen Georges-Pompidou, Paris, France; (2) Service d'Explorations Fonctionnelles rénales et métaboliques, AP-HP Hôpital Européen Georges-Pompidou, Paris, France

Contact : rosa.vargas@egp.aphp.fr

Introduction : Le syndrome de Gitelman est une tubulopathie héréditaire à transmission autosomique récessive avec une fréquence estimée à $1 / 40000$ dans la population caucasienne. Elle est due à des mutations du gène SLC12A 3 codant pour le cotransporteur $\mathrm{Na} / \mathrm{Cl}$ sensible aux thiazides du tube distal. Patients et Résultats: Nous avons étudié une famille française non-consanguine avec 5 individus atteints sur deux générations. Les deux premiers individus étudiés étaient un fils (cas index) et sa mère, tous les deux porteurs d'un tableau clinique univoque de syndrome de Gitelman (hypokaliémie d'origine rénale, hyperaldostéronisme et hyperrénisme secondaires, hypomagnésémie et hypocalciurie). L'analyse par séquençage direct des exons et jonctions exon-intron du gène SLC12A3 a permis la détection de 2 mutations à l'état hétérozygote chez le cas index (p.Ser402X et c. $2747+1 \mathrm{G}>\mathrm{A}$ ) et de 3 mutations également hétérozygotes chez la mère (p.Tyr602CysfsX31, c. $2660+1 G>A$ et c. $2747+1 G>A$ ). Par la suite, l'analyse des autres individus atteints et non atteints a permis de montrer les génotypes suivants : l'oncle et la tante maternels atteints sont porteurs des 3 mêmes mutations que la mère (p.Tyr602CysfsX 31 , c. $2660+1 G>A$ et c. $2747+1 \mathrm{G}>\mathrm{A}$ ), la sœur atteinte est porteuse de 3 mutations hétérozygotes (p.Tyr602CysfsX31, c.2660+1G >A et p.Ser402X), le père bien portant de la mutation p.Ser $402 X$ et la sœur bien portante de 2 mutations hétérozygotes (p.Tyr602CysfsX31 et c. $2660+1 \mathrm{G}>\mathrm{A}$ ). Parmi les mutations détectées, seulement la mutation p.Ser402X a été décrite précédemment dans une famille italienne, les 3 autres mutations sont nouvelles. Conclusions et discussion L'analyse de la ségrégation des 4 mutations détectées dans cette famille a permis de conclure à : la présence de 2 de ces mutations sur le même allèle (p.Tyr602CysfsX31 et c.2660+1G>A), à l'union d'une femme hétérozygote composite avec un porteur sain hétérozygote ce qui explique la transmission pseudo-dominante et la présence des génotypes différents chez le cas index et sa sœur atteinte. La fréquence des hétérozygotes dans la population générale est estimée à $1 / 100$, la probabilité d'avoir un enfant atteint quand on est atteint est donc de 1/200. Cette famille illustre l'importance d'une bonne caractérisation phénotypique et 
génotypique dans l'analyse de maladies récessives qui présentent une transmission verticale. Dans ces cas la probabilité d'une union d'un individu atteint avec un hétérozygote est à considérer, ce qui permet d'effectuer un conseil génétique adéquat.

\section{P512. ADENINE PHOSPHORIBOSYLTRANSFERASE (APRT) DEFICIENCY : PHENOTYPE AND GENOTYPE CHARACTERI- ZATION OF A LARGE COHORT}

G. Bollée (1), C. Dollinger (2), D. Guillemot (2), L. Boutaud (2), A. Bensman (4), J. Harambat (5), P. Deteix (6), M. Daudon (3), B. Knebelman $(1,7)$, I. Ceballos-Picot (2)

(I) Université Paris Descartes; APHP, Department of Nephrology, Necker-Enfants Malades Hospital, Paris, France; (2) Université Paris Descartes; APHP, Laboratory of Metabolic Biochemistry, Necker-Enfants Malades Hospital, Paris, France ; (3) Université Paris Descartes; APHP, Laboratory of Biochemistry, Necker-Enfants Malades Hospital, Paris. France ; (4) Université Paris 6 ; APHP, Department of Pediatric Nephrology, Trousseau Hospita, Paris, France ; (5) Bordeaux Universitary Hospital, Department of Pediatric Nephrology, Bordeaux, France ; (6) Clermont-Ferrand Universitary Hospital, Department of Nephrology, Clermont-Ferrand, France; (7) Inserm U845, Necker-Enfants Malades Hospital, Paris, France

Contact : irene.ceballos@nck.aphp.fr

Adenine phosphoribosyltransférase (APRT) deficiency is a rare autosomal recessive disorder causing 2,8 dihydroxyadénine (2,8-DHA) stones and renal failure secondary to intratubular crystalline precipitation. The disease can be efficiently treated by allopurinol. Data on clinical presentation of APRT deficiency are very limited, especially in caucasian population. We retrospectively reviewed all cases of APRT deficiency identified at Necker Hospital, Paris, France, between 1978 and 2009. Diagnosis of complete APRT deficiency was made in 53 patients from 43 families, at a median age of 28.9 (5.6-51) years. Full clinical data were available in 40 patients from 33 families. A striking finding was that diagnosis was delayed for years from onset of symptoms in many patients. Fourteen $(35 \%)$ patients had decreased renal function at diagnosis. Six $(15 \%)$ patients had reached end stage renal disease (ESRD) and APRT deficiency was detected once disease recurred in renal transplant. Eight patients $(20 \%)$ reached ESRD over follow-up (median duration 74 (14-112) months). Sequencing of aprt gene was performed in 31 families. Fifty-four mutated chromosomes were found on the 62 chromosomes analyzed $(87 \%)$. Eighteen different mutations were identified, 14 of which being novel. A single $T$ insertion at the intron 4 splice donor site (IVS4+2insT) leading to a truncated protein, accounted for $40.3 \%$ of mutations. IVS4+2insT mutation was found in 2 out of $204(0.98 \%)$ chromosomes of healthy newborns. Our report, the largest series reported, highlights the underdiagnosis and the potential severity of APRT deficiency. Early recognition of the disease is crucial to prompt treatment and prevent renal complications.

Mots-clés : adenine phosphoribosyltransferase, lithiasis.

口P513. NOUVELLE STRATÉGIE D'EXPLORATION MOLÉCULAIRE DES REINS POLYKYSTIQUES FETTAUX ET PÉDIATRIQUES : ANALYSER TCF2 AVANT PKHD1

L. Michel-Calemard (1, 2), A. Liutkus (2), B. Ranchin (2), P. Cochat (2, 7), M.P. Cordier (3), M.P. Lavocat (4), F. Nobili (5), R. Bouvier (6), Y. Morel $(1,7)$

(I) Hospices Civils de Lyon, Endocrinologie Moléculaire et Maladies Rares, CBPE, Bron, France; ; (2) Hospices Civils de Lyon, Centre de Référence des Maladies Rénales Rares, Néphrogones et Service de Néphrologie Pédiatrique, HFME, Bron, France ; (3) Hospices Civils de Lyon, Service de Génétique, HFME, Bron, France; (4) Service de Pédiatrie. CHU Hôpital Nord, Saint-Étienne, France; (5) Service de Pédiatrie, CHU Hôpital Saint-Jacques, Besançon, France; (6) Hospices Civils de Lyon, Centre de Pathologie Est, CBPE, Bron, France; (7) Université Lyon 1 , Faculté de médecine Lyon-Est, Lyon, France

Contact : laurence.michel@chu-lyon.fr

HISTORIQUE En 1999, nous avons pris en charge à Lyon le diagnostic moléculaire de la polykystose hépatorénale autosomique récessive (ARPKD). 159 familles (168 patients) provenant de France et de l'étranger ont été étudiées. Depuis la découverte du gène PKHD1 (2002), le séquençage des 67 exons a été progressivement mis en place, mais le diagnostic étant rarement confirmé cliniquement, la lourdeur d'un séquençage exhaustif chez des patients au diagnostic incertain se pose. Une fréquence élevée d'anomalies rénales, notamment kystiques, dues à des mutations du gène HNF1b-TCF2 a été rapportée chez des enfants et des fœtus (Ulinski 2006, Decramer 2007). Les 2 gènes en cause PKHD1, codant pour une mutations sont majoritairement ponctuelles, privées, disséminées dans le gène. TCF2, codant pour un facteur de transcription impliqué dans le diabète MODY 5 (transmission autosomique dominante) et responsable d'anomalies rénales (syndrome RCAD : Renal Cyst And Diabete), comporte 9 exons. Les mutations sont majoritairement des délétions hétérozygotes complètes du gène : les techniques quantitatives (MLPA/QMPSF) permettent de détecter plus de $70 \%$ des mutations responsables. RÉSULTATS Gène PKHD1 : 124 patients (103 familles) ont au moins une mutation PKHD1. Diagnostic fait en anténatal chez 62 patients $(50 \%)$. Signes par ordre de fréquence : néphromégalie oligo-anamnios, reins hyperéchogènes. 42 décès ( 32 ITG, 20 décès néonatals DNN). Gène TCF2: étude rétrospective, 56 familles adressées pour ARPKD sans mutation PKHD1, 8 mutations TCF2 identifiées: 6 délétions complètes, 2 mutations ponctuelles. Depuis 2008, étude prospective, 70 patients adressés pour TCF2, 11 mutations identifiées: 5 délétions complètes, 1 duplication partielle, 5 mutations ponctuelles. Diagnostic fait en anténatal chez 16 patients $(84 \%)$ : majorité de reins hyperéchogènes (13), seulement 3 néphromégalies, 2 anomalies de la quantité de LA. 2 décès (1 ITG, 1 DNN). Les caractères orientant le diagnostic semblent être la taille des reins et l'oligoamnios. Stratégie proposée Depuis 2008, nous appliquons une nouvelle stratégie d'exploration des reins kystiques ou hyperéchogènes fœtaux ou pédiatriques : 1) Étude de TCF2 : recherche de délétion par MLPA, séquençage des 9 exons en cas d'absence de large lésion; 2) Séquençage de PKHD1 en cas d'absence d'anomalie TCF2 et phénotype compatible. Cette démarche diagnostique est modulée par l'histoire familiale et l'examen anatomopathologique. CONCLUSION La nouvelle stratégie semble intéressante pour 2 raisons principales : 1) optimiser la prise en charge des patients, notamment anténatale (pronostic plus sévère dans ARPKD) 2) optimiser l'analyse génétique en évitant l'étude longue et coûteuse de PKHD1 dans un certain nombre de cas. Malgré cette stratégie, certains génotypes restent encore non élucidés. L'étude d'autres gènes, dont PKD1 et PKD2, devrait être envisagée.

Mots-clés : reins hyperéchogènes, TCF2/HNF1b, PKHD1.

\section{P514. DES MUTATIONS DOMINANTES DU GENE CODANT POUR LA RÉNINE SONT RESPONSABLES DE NÉPHROPA- THIES TUBULO-INTERSTITIELLES CHRONIQUES AVEC HYPERURICÉMIE ET ANÉMIE}

V. Morinière (1, 2), O. Gribouval (3), G. Bollée (4), A. Pawtowski (1), M. Matignon (5), C. Loirat (6), C. Maynard (7), P. Niaudet (8), M.C. Gubler (3), C. Antignac (1, 3)

(1) APHP, Département de Génétique, Hôpital Necker, Paris, France ; (2) APHP, Centre de référence des maladies rénales héréditaires de l'enfant et de l'adulte (MARHEA), Hôpital Necker, Paris, France; (3) Inserm U574, Université Paris Descartes, Hopital Necker, Paris, France; (4) APHP, Service de Néphrologie Adulte, Hôpital Necker, Paris, France ; (5) APHP, Service de Néphrologie, Hôpital Henri Mondor et Université ParisXII, Créteil, France; (6) APHP. Service de Néphrologie, Hôpital Robert Debré, Paris, France ; (7) Service de Néphrologie, Centre Hospitalier de Chambéry, France; (8) APHP, Service de Néphrologie Pédiatrique, Hôpital Necker, Paris, France

Contact : vincent.morinier@inserm.fr

Des mutations inactivatrices des gènes codant les différents composants du système rénine angiotensine sont la cause de la dysgénésie tubulaire rénale autosomique récessive, néphropathie sévère du foetus se manifestant principalement par une anurie précoce et persistante responsable le plus souvent de mort périnatale. Récemment, nous avons participé à la caractérisation de mutations dominantes du gène REN codant la rénine chez des patients présentant une néphropathie tubulo-interstitielle chronique avec hyperuricémie et, chez les enfants atteints, anémie sensible à l'érythropoḯtine (Kmoch et al., Am J Hum Genet, 2009). Les deux mutations détectées dans les trois familles entrainaient soit la délétion soit la substitution de la leucine en position 16 dans le peptide signal, provoquant une altération de la translocation de la pré-prorénine dans le réticulum endoplasmique avec comme corollaire une diminution de la production de rénine chez les patients. Il a été montré de plus que la mutation p.Leul6del provoque un stress du réticulum endoplasmique qui pourrait ainsi expliquer l'effet dominant négatif de ces mutations sur la viabilité des cellules exprimant la rénine. Du fait des similarités phénotypiques avec la néphropathie hyperuricémique familiale due à des mutations du gène UMOD codant l'uromoduline, nous avons séquencé l'exon 1 du gène REN codant pour le peptide signal de la pré-pro-rénine, chez 141 individus non apparentés présentant une néphropathie tubulo-interstitielle chronique sans mutation du gène UMOD. Une histoire familiale évocatrice d'une transmission autosomique dominante était présente dans 97 cas et la notion d'hyperuricémie ou de goutte chez le patient ou un membre de sa famille dans 90 cas. Dans 3 cas, des mutations faux sens modifiant la structure du peptide 
signal ont été identifiées, non retrouvées chez plus de 100 contrôles. Il s'agit dans un cas de la mutation p.Leul6Arg déjà identifiée dans une famille, et dans les autres cas des mutations p.Trp17Arg et p.Cys20Arg considérées comme pathogènes par le logiciel Polyphen (scores 3,7 et 3,6 respectivement) et altérant le profil d'hydrophobicité du peptide signal. Comme dans les cas décrits précédemment, les trois patients présentent des antécédents familiaux de néphropathie hyperuricémique, une hyperuricémie (avec crises de goutte à l'âge de 26 ans chez une patiente) et une anémie pour les deux cas détectés dès l'enfance. Au total, ces résultats montrent l'implication de mutations du gène de la rénine dans différentes pathologies rénales, soit dans le cadre de formes autosomiques récessives, pour la dysgénésie tubulaire rénale, soit dans des formes autosomiques dominantes dans les néphropathies tubulointerstitielles chroniques avec hyperuricémie. Il reste à comprendre le mécanisme exact par lequel ces mutations provoquent la symptomatologie et si des mutations situées dans d'autres exons pourraient avoir un effet similaire.

Mots-clés : rénine, néphropathie tubulo-interstitielle chronique, hyperuricémie,

GP515. A NEW FAMILY WITH FAMILIAL HYPERKALAEMIC HYPERTENSION : CLINICAL AND MOLECULAR INVESTIGATION G. Beaurain (1, 2), E. Elvira-Matelot (1), J. Potier (3), A. Blanchard (4), M. Vallet (5), J. Hadchouel (1), P. Houillier $(5,6)$, X. Jeunemaitre (1, 2, 6)

(1) INSERM, Unité 970, PARCC HEGP, Paris, France; (2) AP-HP, Hôpital Européen Georges-Pompidou, Paris, France; (3) Hôpitaux de Cherbourg, Service de Néphrologie, Cherbourg, France; (4) AP-HP, Centre d'Investigation Clinique, Hôpital Européen Georges-Pompidou, Paris, France : (5) AP-HP, Service d'Explorations Fonctionnelles, Hôpital Européen Georges-Pompidou, Paris, France; (6) Université Paris Descartes, Faculté de Médecine, Paris, France, France

Contact : xavier.jeunemaitre@inserm.fr

Introduction: Mutations in the WNK1 and WNK4 genes have been shown to be responsible for familial hyperkalemic hypertension (FHHt, also called PHA2 and Gordon syndrome). Up to now, only two families with WNK1 intron 1 deletion have been described (Wilson. Science 2001). Intron 1 deletion leads to misregulation of the renal expression of the two main L-WNK1 and KS-WNK1 isoforms (Delaloy, Hypertension 2008). Objective : to test the possibility of other rearrangements at the WNK1 locus in FFHt. Patients and Methods: We analyzed 37 index patients (age : $2-50$ years) with suspected $\mathrm{FHHt}$, based on the presence of sustained and unexplained hyperkalemia $(5.2-8.5$ $\mathrm{mmol} / \mathrm{L})$, hyperchloremia $(98-115 \mathrm{mmol} / \mathrm{L})$, a variable metabolic acidosis, and increased blood pressure. Genomic DNA was amplified, $\mathrm{Cy}$ labeled and hybridized on a dedicated DNA chip (Nimblegen CGH customed array, 67893 unique probes) covering the $160 \mathrm{~Kb}$ WNK1 locus plus $290 \mathrm{~Kb}$ upstream and $100 \mathrm{~Kb}$ downstream. The results were analyzed using the Nimblegen Signal Map software. This genetic analysis was also performed in 40 healthy controls. Results : Several copy number variants at the WNK1 locus were detected, some of them being already described, one being present in 8 affected individuals but not in controls. A new $23.1 \mathrm{~Kb}$ deletion in intron 1 was detected in one case, limiting the minimum deleted interval to $18 \mathrm{~Kb}$. This interval contains 4 out the 5 regulatory elements previously shown in vitro to affect WNK1 gene expression. In vitro analysis demonstrated that one of this element acts as an insulator, preventing interactions between the regulatory elements of L-WNK1 and KS-WNK1. The FHHt case corresponded to a 4-generation family which was further investigated. All positive individuals presented classical features of $\mathrm{FHHt}$, except two children $<12$ years being not hypertensive. All were remarkably sensitive to small doses of thiazides. No hypercalciuria was observed on ad-libitum diet. An intravenous calcium challenge test confirmed the normal regulation of $\mathrm{Ca}++$ in the distal tubule, contrary to mutations affecting the WNK4 gene. Conclusion: We found a new FHHt family with a $23.1 \mathrm{~Kb}$ deletion in the intron 1 of the WNK1 gene, overlapping the two deletions previously observed. This deletion confirms the importance of intron 1 regulatory sequences in regulating WNK1 gene expression and in causing FHHt when deleted. The presence of other genomic rearrangements in FHHt subjects might indicate the possibility of regulatory mutations which functionality remains to be established.

Mots-clés : hypertension, délétion, kinase.

aP516. LE SPECTRE CLINIQUE DU SYNDROME DE COCKAYNE: DONNÉES CLINIQUES ET MOLÉCULAIRES À PARTIR D'UNE COHORTE DE 50 PATIENTS

V. Laugel $(1,2)$, C. Dalloz (1), M. Durand (1), A. Zaloszyc (1, 2), M.C. Vincent (3), L. Pasquier (4), S. Odent (4), V. Cormier-Daire (5), B. Gener (6),
E.S. Tobias (7), D. Martin-Coignard (8), V. Drouin-Garraud (9), D. Heron (10), H. Journel (11), J. Vigneron (12), S. Lyonnet (5), D. Gubser-Mercati (13), B. Funalot (14), L. Brueton (15), J. Sanchez Del Pozo (16), E. Muñoz (17), A.R. Gennery (18), K. Prescott (19), L. Ramos (20), Z. Stark (21), K. Fieggen (22), P. Sarda (23), P. Edery (24), A. Megarbane (25), A. Bloch-Zupan (26), A. Sarasin (27), H. Dollfus (1)

(I) Laboratoire de Génétique Médicale, Faculté de Médecine, Strasbourg, France; (2) Service de Pédiatrie 1, CHU Strasbourg, France; (3) Laboratoire de Diagnostic Génétique, CHU Strasbourg, France ; (4) Service de Génétique Médicale, CHU Rennes, France; (5) Service de Génétique Médicale, Hôpital Necker-Enfants Malades, Paris, France; (6) Department of Medical Genetics, Cruces Hospital, Baracaldo, Vizcaya, Spain : (7) Division of Developmental Medicine. The Royal Hospital for Sick Children, Glasgow, UK; (8) Service de Génétique Médicale, CHU Le Mans, France: (9) Service de Génétique Médicale, CHU Rouen, France ; (10) Service de Génétique Médicale, Hôpital Pitié-Salpêtrière, Paris, France: (11) Service de Génétique Médicale, Hôpital Bretagne-Atlantique, Vannes, France ; (12) Service de Pédiatrie, Maternité A. Pinard, Nancy, France ; (13) Neurologie Pédiatrique, Neuchâtel, Suisse; (14) Service de Neurologie, CHU Limoges, France; (15) Department of Clinical Genetics, Women's Hospital, Birmingham, UK; (16) Department of Genetics, Hospital I2 de Octubre, Madrid, Spain; (17) Department of Neurology, Hospital Clinic, Barcelona, Spain ; (18) Department of Pediatrics, Newcastle General Hospital, Newcastle upon Tyne, UK ; (19) Department of Clinical Genetics, St James's University Hospital, Leeds, UK : (20) Department of Medical Genetics, Pediatric Hospital, Coimbra, Portugal; (21) Genetic Health Services Victoria, Melbourne, Australia: (22) Department of Medical Genetics, UCT Faculty of Health Sciences, Groote Schuur and Red Cross Children's Hospital, Cape Town, South Africa : (23) Service de Génétique Médicale, CHU Montpellier, France; (24) Service de Génétique Clinique, Hôpital Femme-Mère-Enfant, Lyon, France : (25) Unité de Génétique Médicale, Université Saint-Joseph, Beyrouth, Liban: (26) Service d'Odontologie, CHU Strasbourg, France; (27) CNRS FRE2939, IGR, Villejuif, France

Contact : vincent.laugel@chru-strasbourg.fr

Le syndrome de Cockayne est une maladie multisystémique complexe liée à une anomalie de la réparation et de la transcription de l'ADN. L'incidence de ce syndrome a été estimée à 1/300 000 naissances en Europe Occidentale, mais reste probablement sous-évaluée. Les patients présentent généralement un retard de croissance, une microcéphalie, un retard de développement psychomoteur, une atteinte sensorielle. Le spectre du syndrome de Cockayne comprend un large éventail de présentations cliniques en termes de sévérité et d'âge de début, depuis des formes prénatales sévères jusqu'à des formes modérées de révélation parfois très tardives. Il n'y a pas de corrélation simple entre la sévérité clinique d'une part et l'anomalie cellulaire ou moléculaire d'autre part. Nous avons collecté les données cliniques et moléculaires d'une cohorte internationale de 50 patients Cockayne démontrés sur le plan génétique. Nos données indiquent que les présentations cliniques classiques du syndrome de Cockayne (types I-IIIII) forment en réalité un continuum de sévérité, même si l'identification des différents sous-groupes reste utile dans la pratique quotidienne pour le diagnostic positif et le pronostic à long terme. Le syndrome COFS (cérébro-oculo-facio-squelettique) doit être considéré comme une forme prénatale de syndrome de Cockayne mais nécessite des critères diagnostiques cliniques spécifiques. À l'autre extrémité du spectre, nous rapportons le cas clinique d'un patient décédé à 57 ans et présentant une forme particulièrement tardive de ce syndrome. L'imagerie cérébrale du syndrome de Cockayne montre classiquement la présence de calcifications des noyaux gris, d'anomalies de la substance blanche et d'une atrophie cérébelleuse. Nous avons étudié prospectivement et rétrospectivement les imageries cérébrales de 20 patients Cockayne, afin d'identifier les éléments les plus caractéristiques pour le diagnostic et d'en préciser l'histoire naturelle. Nous avons répertorié les 45 mutations publiées à ce jour pour les gènes CSA et CSB responsables du syndrome de Cockayne et nous rapportons 43 nouvelles mutations dans ces mêmes gènes. Parmi les 84 familles connues, $52(62 \%)$ présentent une mutation dans le gène CSB. Il existe un recouvrement important entre les symptômes cliniques des patients CSA et CSB mais les formes les plus sévères et les plus précoces de syndrome de Cockayne semblent liées au gène CSB. La plupart des mutations faux-sens sont regroupées dans certains domaines fonctionnels de ces protéines et offrent des pistes pour mieux comprendre leur mécanique moléculaire. 
口P517. ÉTUDE CLINIQUE, PHYSIQUE ET GÉNÉTIQUE DU SYNDROME DE COCKAYNE DANS UNE FAMILLE TUNISIENNE

M. Gribaa (1), R. Bhouri (1), L. Adela (1), A. Ben Elkadhi (2), H. ElGhezal (1), V. Laugel (4), I. Ben Charfeddine (1), O. Mamai (1), A. Mili (1), T. Belazreg (1), D. H'mida (1), A. Sarasin (3), A. Saad (1)

(1) Laboratoire de Cytogénétique, de Génétique Moléculaire et de Biologie de la Reproduction Humaines, CHU Farhat Hached, Sousse, Tunisie ; (2) Pédiatrie, Cabinet Privé, Hammamet, Tunisie ; (3) Unité de Stabilité Génétique et Oncogenèse CNRS FRE 2939, Institut Gustave Roussy, Villejuif, France ; (4)Service de Neuropédiatrie/Pédiatrie 1, CHU Strasbourg-Hautepierre, Strasbourg, France

Contact : r.bhouri@gmail.com

Le syndrome de Cockayne est une maladie multisystémique rare caractérisée par un retard staturo-pondéral, une dysmorphie faciale, des troubles neurologiques progressifs avec déficit intellectuel et une photosensibilité. Il appartient au groupe des maladies affectant la réparation de l'ADN par excision de nucléotides. Il est caractérisé par grande variabilité dans la sévérité et l'âge d'apparition des manifestations cliniques. Sa transmission est autosomique récessive. Des mutations ont été identifiées au niveau des gènes ERCC6 (CSB ; 10q11) ERCC8 (CSA ; 5q12.1) mais aucun lien entre les sous-types de la maladie et les gènes impliqués n'a été à ce jour établi. Nous avons étudié le cas d'une fillette de 6 ans issue de parents consanguins au $2^{e}$ degré. Elle présente un retard staturo-pondéral, psychomoteur et intellectuel sévères avec surdité de perception bilatérale et une dysmorphie faciale évocatrice du syndrome de Cockayne de type I. La mesure de l'efficacité de transcription au niveau des fibroblastes de peau mis en culture a objectivé un déficit important de la récupération de la synthèse de l'ARN après irradiation des cellules par les UVC confirmé la présence d'une anomalie de réparation de l'ADN. Ce résultat, en association avec le tableau clinique, est fortement évocateur du syndrome de Cockayne. Une analyse moléculaire est en cours pour la recherche d'une éventuelle mutation dans le gène responsable pour confirmer le diagnostic et proposer un conseil génétique adéquat à cette famille.

Mots-clés : Cockayne, troubles neurologiques, réparation de l'ADN.

שP518. UNE NOUVELLE MUTATION DU GENE WRN RESPONSABLE DU SYNDROME DE WERNER CHEZ UN PATIENT TUNISIEN

O. Mamaï (1), M. Gribaa (1), L. Bouzouffara (2), N. Uhrhammer (3), L. Adala (1), I. Ben Charfeddine (1), T. Belazreg (1), A. Mili (1), C. Belajouza (2), M. Denguezli (2), Y.J. Bignon (3), A. Saad (1)

(1) Laboratoire de Cytogénétique, de Génétique Moléculaire et de Biologie de la Reproduction Humaines, CHU Farhat Hached, Sousse, Tunisie ; (2) Service de Dermatologie et de Vénérologie, CHU Farhat Hached, Sousse, Tunisie; ; (3) Laboratoire de Diagnostic Génétique et Moléculaire, Centre Jean Perrin, Clermont-Ferrand, France

Contact : moez.gribaa@rns.tn

Le syndrome de Werner est une maladie héréditaire ayant une transmission autosomique récessive. Il est caractérisé par une accélération du développement de plusieurs tissues de l'organisme. Il est secondaire à la mutation du gène WRN qui code pour la protéine WRN qui une activité exonucléase et hélicase. Dans ce travail, nous avons étudié un patient tunisien âgé de 43 ans, issu de parents consanguins au premier degré. Cliniquement, il a présenté un aspect sclérodermiforme de la peau, des signes de vieillissement cutané précoce avec dysmorphie faciale (faciès d'oiseau), des ulcérations chroniques des 2 jambes et une cataracte bilatérale. Le séquençage direct de toute la partie codante de son gène WRN a permis de retrouver une délétion homozygote de 25 nucléotides entre les nucléotides 3039 et 3063. Cette mutation est localisée dans le domaine responsable de l'activité hélicase de la protéine. Elle est, par ailleurs, responsable d'un décalage du cadre de lecture et la création d'un codon stop prématuré à l'origine d'une protéine tronquée. Cette nouvelle mutation, est sans doute responsable de la pathologie de ce patient et a permis de confirmer son atteinte par le syndrome de Werner.

Mots-clés : gène WRN, syndrome de Werner, nouvelle mutation.

- 519 . MUTATIONS DU GÈNE UPF3B ASSOCIÉES À UNE DÉFICIENCE MENTALE NON SPÉCIFIQUE AVEC OU SANS AUTISME

F. Laumonnier (1), C. Shoubridge (2), C. Antar (1, 3), L.S. Nguyen (2), H. Van Esch (4), T. Kleefstra (5), S. Briault (6), J.P. Fryns (4), B. Hamel (5), J. Chelly (7), H.H. Ropers (8), N. Ronce (1, 3), S. Blesson (3), C. Moraine (1), J. Gecz $(2,9)$, M. Raynaud $(1,3)$

(1) INSERM U930, Université François-Rabelais, CNRS ERL 3106,Tours,
Hospital, North Adelaide, SA, Australia ; (3) Centre Hospitalier Régional Universitaire, Tours, France ; (4) Center for Human Genetics, University Hospitals Leuven, Leuven, Belgium; (5) Department of Human Genetics, Radboud University Nijmegen Medical Centre, Nijmegen, The Netherlands: (6) Centre Hospitalier Régional, Orléans, France; (7) Institut Cochin, University Paris-Descartes, CNRS UMR 8104, INSERM U567, Paris, France ; (8) Department of Human Molecular Genetics, Max Planck Institute for Molecular Genetics, Berlin-Dahlem, Germany ; (9) Schools of Paediatrics, Reproductive Health, Molecular and Biomedical Science, University of Adelaide, Adelaide, SA, Australia

Contact : frederic.laumonnier@univ-tours.fr

Des mutations dans le gène UPF3B, qui code une protéine impliquée dans la voie de dégradation des ARNm à codon stop prématuré, ont été récemment caractérisées chez 4 familles présentant une déficience mentale liée au chromosome X (DMX) spécifique (syndromes FG et de Lujan-Fryns), non spécifique avec ou sans autisme. Afin de mieux déterminer la contribution de ce gène dans la déficience mentale, nous avons analysé sa séquence codante chez 397 familles collectées par le consortium EuroMRX. Nous avons identifié 1 mutation non-sens, c. $1081 \mathrm{C}>\mathrm{T} / \mathrm{p}$.Arg361*, dans une famille incluant des garçons atteints de DMX non spécifique (MRX62), et 2 mutations faux-sens chez 2 autres familles dans lesquelles les patients ont une DM avec ou sans autisme (c.1136G > A/p.Arg379His et c.1103G > A/p.Arg368Gin). Des études fonctionnelles effectuées par RT-PCR quantitative et Western blot sur les lignées lymphoblastoides des patients ont montré que la mutation c. $1081 \mathrm{C}>\mathrm{T}$ aboutissait à une dégradation de l'ARNm d'UPF3B et à l'absence de la protéine UPF3B. Nous avons également étudié la localisation subcellulaire des formes normale et mutées de la protéine UPF3B après transfection dans des cultures primaires de neurones hippocampaux d'embryon de souris. Nous n'avons pas constaté de différence significative entre les différentes formes, en revanche nous avons montré que la protéine UPF3B est exprimée dans le corps cellulaire, l'axone, les dendrites et les épines dendritiques, lesquelles sont essentielles pour la neurotransmission et les processus d'apprentissage et de mémoire. Nos résultats démontrent qu'en plus des syndromes FG et Lujan-Fryns, des mutations tronquantes de la protéine peuvent causer une DM non spécifique et de l'autisme. La localisation neuronale, en particulier synaptique, de la protéine UPF3B et sa fonction essentielle dans la surveillance des ARNm suggèrent un rôle potentiel dans la régulation de l'expression et de la dégradation des ARNm présents au niveau de la synapse.

Mots-clés : autisme, déficience mentale, chromosome X.

口P520. DÉLÉTION DU GÈNE FMR1: QUAND LA GÉNÉTIQUE MOLÉCULAIRE ET LA CYTOGÉNÉTIQUE FONT BON MÉNAGE

N. Boutry-Kryza (1), M.P. Cordier (2), A. Labalme (3), M. Till (2, 3), P. Edery (2, 3), A. Calender (1), D. Sanlaville (2, 3), G. Lesca $(1,2)$ (1) Laboratoire de Génétique Moléculaire, Hôpital Édouard Herriot, Hospices Civils de Lyon, France; (2) Service de Génétique Clinique, Hôpital Femme-Mère-Enfant, Hospices civils de Lyon, France; (3) Service de Cytogénétique Constitutionnelle, Centre de Biologie et de Pathologie Est. Hospices Civils de Lyon, France

Contact : nadia.kryza@chu-lyon.fr

Le syndrome de $\mathrm{I}^{\prime} \mathrm{X}$ fragile est la $\mathrm{I}^{\mathrm{r}}$ cause de retard mental héréditaire. Dans la majorité des cas, l'anomalie responsable est une expansion d'un triplet nucléotidique dans la partie 5'UTR du gène FMR1 situé en Xq27.3. Le diagnostic est posé par la mise en évidence de cette expansion par la technique de Southern blot. Cependant, dans de rares cas, l'anomalie causale est une délétion d'une partie ou de la totalité du gène. On distingue 2 types de délétions: les délétions de petite taille, inférieures à $10 \mathrm{~kb}$, emportant principalement la partie 5'UTR et l'exon 1 du gène et survenant généralement sur un allèle prémuté au cours d'une méiose maternelle. II existe également des délétions de plus grande taille emportant le gène FMR1 et certains gènes adjacents selon la taille de la délétion. Enfin, 3 cas de mutations ponctuelles ont également été décrits. Les délétions et mutations ponctuelles ne sont, quant à elles, pas toutes détectables par les techniques classiquement utilisées pour le diagnostic du syndrome de l'X fragile. Nous rapportons le cas de Hugo, 5 ans, consultant pour retard mental sans antécédent familial particulier. Ses antécédents personnels sont marqués par un développement psychomoteur anormal. Lors de l'examen, le patient présente un retard de langage associé à des troubles du comportement à type d'hyperactivité, de déficit de l'attention et d'angoisse. Sur un plan morphologique, les oreilles sont grandes et il existe un rétrognatisme. Un syndrome de l'X fragile est donc recherché. Sur le Southern blot à la recherche d'une expansion, nous n'obtenons aucun signal sur 2 prélèvements indépendants laissant suspecter une délétion du gène FMR1. Un 
examen par CGH microarray est réalisé et montre la présence d'une délétion en position Xq27.3 emportant le gène FMR1 et un gène voisin, de fonction inconnue, le gène FMRInb. Ce résultat est confirmé par hybridation in situ en fluorescence. Le séquençage du point de cassure montre une délétion d'environ $270 \mathrm{~Kb}$ avec un point de cassure proximal situé en position 146.791.349 (hg 18) dans un élément line (L1MC2) et un point de cassure distal en position 147.061.104 (hg 18) au début d'un autre élément line (L1PA4). L'étude familiale retrouve la délétion chez la mère mais ne montre ni délétion ni prémutation chez les grands-parents maternels. L'étude des microsatellites a montré que la délétion s'est produite lors du passage du chromosome $\mathrm{X}$ grand paternel vers sa fille probablement par recombinaison inégale entre les 2 éléments de type line. Au final, nous avons diagnostiqué un syndrome de l' $\mathrm{X}$ fragile par délétion de grande taille du gène FMRI. Cette délétion, héritée de la mère, modifie le conseil génétique. Il existe un risque de récurrence de $50 \%$ pour une future grossesse s'il s'agit d'un garçon et en cas d'examen prénatal, la recherche de délétion s'effectuera par une technique cytogénétique et non par Southern blot. Ce cas illustre une fois de plus la complexité du diagnostic de l'X fragile.

Mots-clés : $\mathrm{X}$ fragile, délétion.

\section{IP521. RETARD MENTAL DÛ À UNE DUPLICATION EN AMONT} DU GENE GRIA3

C. Bonnet (1, 2), M. Béri (1, 2), P. Callier (3), F. Mugneret (3), A. Perrin (1), L. Faivre (4), P. Jonveaux $(1,2)$

(1) Laboratoire de Génétique, CHU Nancy, Vandauvre les Nancy, France; (2) Nancy Université, EA 4368, Laboratoire de Génétique, Centre Hospitalier Universitaire de Nancy, Vandouvre les Nancy, France ; (3) Service de Cytogénétique, CHU, Dijon, France ; (4) Centre de Génétique et Centre de Référence Maladies Rares Anomalies du développement et syndromes malformatifs, Hôpital d'Enfants, Dijon, France

Contact : ce.bonnet@chu-nancy.fr

Nous avons identifié par CGH-array (Agilent 105K), chez un patient présentant une hypotonie axiale, un retard mental sévère, une épilepsie et une dysmorphie faciale, une duplication en Xq24q25 d'une taille de $970 \mathrm{~kb}$ ne contenant pas de gène et située à environ $800 \mathrm{~kb}$ en amont du gène GRIA3 impliqué dans le retard mental lié à l'X. Cette duplication est héritée de la mère du patient qui présente un biais d'inactivation de l'X $(92 \%-8 \%)$. Les études de l'expression du gène GRIA3 par RT-qPCR réalisées sur de I'ARN extrait à partir d'un prélèvement sanguin montrent une forte diminution de l'expression du gène GRIA3 chez le patient par rapport à des témoins. Le niveau d'expression de ce même gène chez la mère porteuse de la duplication Xq24q25 avec un biais d'inactivation de l'X est normal. Le gène GRIA 3 est exprimé au niveau du cerveau et code la sous-unité 3 du récepteur au glutamate. Ce gène est impliqué dans le retard mental lié à l'X: une translocation interrompant le gène, une délétion complète, des duplications partielles et des mutations faux-sens du gène GRIA3 ont été décrites. Ces patients présentent un retard mental parfois associé à des troubles du comportement de type autistique, une épilepsie (un cas) et une dysmorphie faciale. Les femmes porteuses avec biais d'inactivation de l'X sont asymptomatiques. Une femme porteuse d'une duplication intragénique sans biais d'inactivation de l'X et présentant un retard mental a été décrite. Il s'agit du premier cas de duplication située en amont du gène GRIA3 responsable de retard mental chez un garçon par effet de position en inhibant de l'expression du gène. Le réarrangement chromosomique en $\mathrm{Xq} 24 \mathrm{q} 25$ est probablement responsable d'une modification de la configuration tri-dimentionnelle de la chromatine qui modifie l'accessibilité des éléments régulateurs de l'expression du gène.

Mots-clés : retard mental, GRIA3, effet de position.

IP522. UNE NOUVELLE MUTATION AU NIVEAU DU GÈNE PQBPI EST RESPONSABLE DE RETARD MENTAL LIÉ AU CHROMOSOME $X$ CHEZ UNE FAMILLE TUNISIENNE AVEC PETITE TAILLE, MAIGREUR ET MICROCÉPHALIE

L. Ben Jemaa $(1,2)$, I. Rejeb (1), L. Abaied (1), L. Kraoua (1, 2), Y. Sallour (3), F. Maazoul (2), J. Chelly (3), H. Chaabouni (1, 2)

(1) Laboratoire de Génétique Humaine, Faculté de Médecine de Tunis, Tunis, Tunisie; (2) Service des Maladies Héréditaires et Congénitales, Hôpital Charles-Nicole, Tunis, Tunisie; (3) Institut Cochin, Université Paris-Descartes, Inserm U567, UMR 8104, Paris, France

Contact : benjemaal@yahoo.com

Les retards mentaux liés au chromosome X (RMLX) affectent approximativement un garçon sur 600 et sont extrêmement hétérogènes sur le plan génétique. Pour une famille Tunisienne comportant 3 garçons présentant un retard mental (RM) sévère à modéré, petite taille, maigreur et microcéphalie le génotypage du chromosome $\mathrm{X}$ a conclut à une liaison au niveau d'une région allant de DXS8090 à DXS8077 en Xp21.1-Xq21.33, sur la base du tableau clinique présent chez cette famille nous avons décidé de séquencer le gène PQBP1 (Polyglutamin Binding Protein1), ce qui nous a permis d'identifier une nouvelle mutation au niveau de ce gène de type insertion, cette mutation entraîne un décalage du cadre de lecture et une protéine tronquée en effet la protéine mutée se retrouve dépourvue de son domaine $\mathrm{C} 2$, ce qui entraine un défaut de l'épissage des ARNs pré-messagers dans le noyau du fait de la perte d'interaction entre le domaine C2 absent et les protéines impliquées dans la machinerie d'épissage. On propose alors qu'à chaque fois qu'un tableau clinique comportant un RM, petite taille, maigreur et microcéphalie est rencontré chez une fratrie le gène $\mathrm{PQBP} 1$ devrait être séquencé en priorité.

Mots-clés : gène PQBP1, Retard mental lié à 1'X, mutation.

\section{P523. DÉFICIENCE MENTALE LIÉE À L'X ET INACTIVATION COMPLÈTEMENT DÉVIÉE CHEZ LES CONDUCTRICES : QUELS GENES ?}

N. Pasquier (1), N. Ronce (1, 2), M.P. Moizard (1, 2), N. Marmin (1), B. Dessay (1), I. Mortemousque (1), S. Blesson (1), A. Toutain (1), M. Raynaud $(1,2)$

(1) CHRU de Tours, Service de Génétique, Tours, France ; (2) INSERM U930, Tours, France

Contact : m.raynaud@chu-tours.fr

L'inactivation des chromosomes $\mathrm{X}(\mathrm{XCI})$ est le phénomène qui permet de rééquilibrer le contenu en gènes entre les cellules femelles (deux chromosomes X) et les cellules mâles (un chromosome X et un Y) chez les mammifères. Dans la population générale humaine chez les femmes, l'inactivation des X se fait au hasard et le ratio d'inactivation a une distribution normale : la moyenne est de 50:50 et seul un faible pourcentage de femmes (5\%-8\%) a un ratio complètement dévié (plus de $90: 10$ ). La prévalence de la déficience mentale (DM) est de l'ordre de 0,5 à $3 \%$ et, parmi les causes génétiques, l'existence des génopathies liées au chromosome $\mathrm{X}$ explique que l'on observe plus de garçons retardés mentaux que de filles. Le plus souvent, les femmes sont asymptomatiques et susceptibles de transmettre la maladie. Dans certaines familles de DM liée à I'X, les femmes conductrices ont toutes une inactivation complètement déviée et les femmes non conductrices ont une inactivation aléatoire. Les progrès réalisés dans l'identification des gènes et des mutations impliqués dans les retards mentaux liés à l'X permettent d'associer dans un certain nombre de cas l'anomalie causale et la particularité du profil d'inactivation chez les conductrices. Les hypothèses sur les mécanismes qui sont à l'origine de cette particularité seront discutées.

Mots-clés : inactivation des chromosomes $\mathrm{X}$, déficience mentale liée à l'X, retard mental.

\section{IP524. ISODISOMIE PARTIELLE XQ27.3-XQTER CHEZ UNE FEMME PRÉSENTANT UNE HÉMOPHILIE A ET UN RETARD MENTAL LÉGER}

I. Giurgea (1, 2), C. Rothschild (3), P. Boisseau (4), L. Golmard (2), S. Letourneau (1), M. Goossens (1, 2), C. Costa $(1,2)$

(1) Laboratoire de Génétique, CHU Henri-Mondor, Créteil, France; (2) INSERM U955, équipe 11, CHU Henri Mondor, Créteil, France; (3) Centre de Traitement de l'Hémophilie, CHU Necker, Paris, France ; (4) Génétique Médicale et Génétique Moléculaire, CHU Hôtel Dieu, Nantes, France

Contact : irina.giurgea@inserm.fr

L'hémophilie A, maladie liée à l'X caractérisée par un déficit en facteur VIII, a une incidence de 1/5000 naissances masculines, Les hémophilies féminines sont exceptionnelles et rencontrées seulement si à une mutation du gène du facteur VIII (F8) s'associe à un syndrome de Turner, ou une seconde mutation du F8 en trans, ou une inactivation défavorable du chromosome X. Nous rapportons une patiente sans antécédents familiaux particuliers, présentant une forme mineure d'hémophilie A (FVIII c $=13 \%)$ et un retard mental léger, adressée en consultation devant un projet parental de sa fille. L'étude complète du F8 chez elle, a mis en évidence une nouvelle variation c.6268A>C (p.Ile2090Leu) à l'état homozygote. Une hémizygotie a été exclue par l'étude du F8 par MLPA. Les études " in silico » de cette variation et les données de la littérature suggèrent qu'elle pourrait être responsable d'une forme mineure d'hémophilie. Sa fille asymptomatique présente cette variation à l'état hétérozygote. En l'absence de consanguinité et d'hémophilie chez le père, l'hypothèse la plus probable expliquant la présence à l'état homozygote d'un variant rare du F8 chez cette patiente est l'isodisomie maternelle du chromosome X. Afin d'étayer cette hypothèse, nous avons réalisé une étude des marqueurs microsatellites du 
$X$ chez notre patiente et chez sa fille. Chez la patiente, les dix marqueurs situés en Xq27.3-Xqter sont à l'état homozygote (alors qu'ils sont à l'état hétérozygote chez sa fille) suggérant une isodisomie partielle de cette région. A cause de l'inactivation de l'X, chez les femmes, une disomie structurale du chromosome $\mathrm{X}$ ne devrait pas s'accompagner d'une disomie fonctionnelle. Ainsi, se pose la question de l'existence d'un autre événement mutationnel responsable du retard mental présenté par cette patiente. Dans la région Xq27.3-Xqter plusieurs gènes (IDS, ABCD1, SLC6A8, L1CAM, MECP2, FLNA, GDI1, IKBKG et DKC1) ont été impliqués dans le retard mental syndromique ou non. Cependant, la plupart de ces gènes sont associés à un phénotype plus sévère que celui de notre patiente, suggérant que d'autres gènes de cette région pourraient également être impliqués. En conclusion, nous rapportons une femme présentant l'association d'une hémophilie A mineure, probablement lié à une variation rare du F8 et une isodisomie partielle Xq27.3-Xqter et d'un retard mental léger. Le caractère exceptionnel de cette observation et la rareté du mécanisme sousjacent nous incitent à poursuivre la caractérisation moléculaire plus fine de cette isodisomie et à étudier plus attentivement des gènes de cette région possiblement impliqués dans le retard mental.

Mots-clés : hémophilie féminine, retard mental, isodisomie partielle.

\section{I9525. CRIBLAGE DU GENE SLC9A6 PAR HRM DANS UNE COHORTE DE PATIENTS SUSPECTS DE SYNDROME D'ANGELMAN OU DE SYNDROME OLIGOPHRÉNINE}

C. Lacoste (1), M. Bertrand (1), L. Villard (2), N. Levy (1, 2), S. Sigaudy (3), P. Malzac (1), N. Philip (2, 3), A. Moncla $(2,4)$, C. Badens $(1,2)$

(1) Laboratoire de Génétique Moléculaire, Département de Génétique, Hôpital d'enfants de la Timone, Marseille, France; (2) UMR U 910 , Faculté de Médecine, Marseille, France; (3) Service de génétique Clinique, Département de Génétique, Hôpital d'enfants de la Timone, Marseille, France; (4) Laboratoire de cytogénétique, Département de Génétique, Hôpital d'enfants de la Timone, Marseille, France

Contact : catherine.badens@ap-hm.fr

Récemment, 4 mutations dans le gène SLC9A6 ont été décrites chez des patients présentant un retard mental, une microcéphalie, une épilepsie précoce et un phénotype proche de celui du syndrome d'Angelman. Les mutations identifiées entrainent soit la perte de certains acides aminés soit l'apparition d'un codon stop prématuré conduisant à une protéine tronquée, soit des anomalies d'épissage. Le gène SLC9A6 est localisée en Xq26.3 et code pour une pompe $\mathrm{Na}+/ \mathrm{H}+$, NHE6, présente dans la membrane des endosomes. Nous avons recherché des mutations de ce gène dans une cohorte de 53 patients adressés pour une suspicion de syndrome d'Angelman et pour lesquels l'exploration de la région $15 \mathrm{q}$ n'avait pas révélé d'anomalie. Du fait de la présence d'atrophie du cervelet chez 3 des 7 patients rapportés, nous avons également criblé SLC9A6 chez 45 patients adressés pour une recherche de mutation dans Oligophrénine et dont l'analyse avait été négative. Le gène comporte 16 exons et code pour un ARNm de 4454 pb (NM_001042537). Pour l'analyse, il a été fragmenté en 18 amplicons et criblé par la technique de HRM (fusion à haute résolution). Cinq \% des fragments présentaient un profil anormal et ont été séquencés, un quart des fragments séquencés comportaient une variation de séquence. Au total, sur les 98 individus criblés, la technique de HRM a permis de détecter 4 polymorphismes introniques déjà décrits et deux substitutions dans des régions codantes non rapportée dans les bases de données: p.Ala395Ala retrouvée chez un patient suspect de syndrome d'Angelman et p.Ala9Ser identifiée chez 2 patients, l'un suspect de syndrome d'Angelman et l'autre d'Oligophrénine. Aucune de ces deux mutations n'ont été retrouvées après criblage de 120 chromosomes témoins. La substitution p.Ala9Ser a été rapportée récemment chez un patient présentant un syndrome Angelman-like mais était plutôt considérée comme un polymorphisme rare. Des analyses complémentaires pour évaluer l'effet de ces mutations faux sens devraient permettre d'en préciser le potentiel pathogène.

Mots-clés : retard mental, Angelman, oligophrénine.

\section{P526. HÉTÉROGÉNÉITÉ GÉNÉTIQUE DANS LE SYNDROME DE RETT : CARACTÉRISATIONS MOLÉCULAIRE ET CLI- NIQUE CHEZ QUATRE FEMMES HÉTÉROZYGOTES POUR} UNE MUTATION DÉLÉTÈRE DANS LE GËNE FOXGI

L. Lambert (1), L. Allou (1), C. Nemos (1), D. Amsallem (2), C. Francannet (3), P. Edery (4), A. Saunier (1), F. Verneau (1), P. Jonveaux (1), C. Philippe (1)

(1) Laboratoire de génétique médicale, EA 4368, CHRU, Vandauvre-lesNancy, France; (2) Service de neuropédiatrie, Hôpital St Jacques,
Clermont-Ferrand I, France; (4) Unité de Génétique Clinique, Hôpital Femme-Mère-Enfant, Bron, France

Contact : c.philippe@chu-nancy.fr

Le gène FOXG1 a été récemment impliqué dans l'étiologie moléculaire du syndrome de Rett (RTT) dans sa forme congénitale. FOXG1 code une protéine contenant une boîte fork-head, ce facteur de transcription joue un rôle déterminant dans le développement du prosencéphale. À côté du transcrit FOXGIB avec un seul exon, un épissage alternatif apparemment spécifique du cerveau fætal implique 4 exons additionnels. Nous avons réalisé un criblage mutationnel de la totalité des 5 exons par séquençage systématique et QMPSF pour un ensemble de 125 patientes qui présentaient une forme classique, un variant congénital ou de type Hanefeld de RTT. Quinze garçons avec une encéphalopathie épileptique néonatale ou des présentations cliniques RTT-like ont également été analysés. Nous n'avons pas détecté de mutations ponctuelles ou de grands réarrangements affectant les exons 2 à 5 du gène FOXG1. L'implication des transcrits alternatifs dans l'étiologie moléculaire du RTT reste donc à démontrer, il faut cependant noter que les éventuelles protéines résultant de la traduction de ces transcrits n'ont pas été caractérisées à ce jour. Trois variations de séquence délétères entraînant la production de protéines FOXG1 tronquées ont été mises en évidence : deux non-sens (p.Trp308X, p.Tyr400X) et décalage du cadre de lecture très précoce (c.256dupC ; Gln86ProfsX 35 ). Nous avons débuté une analyse pangénomique à l'aide de micro-réseaux 105A (Agilent) chez quelques patientes présentant un RTT typique ou congénital. Nous avons caractérisé une délétion de $2,1 \mathrm{Mb}$ dans la région q12 du chromosome 14 , distale mais très proche du locus du gène FOXG1. La borne distale de cette aneuploïdie segmentaire est située à $40 \mathrm{~kb}$ de l'extrimité 3' de l'exon 5 de FOXG1. Trois patientes sont atteintes d'une forme assez sévère de RTT avec une période de développement normale inférieure à 6 mois. Un allèle délétère (le codon stop prématuré le plus proche de l'extrémité $\mathrm{COOH}$-terminale; p.Tyr400X) est associé à une forme typique de RTT. À notre connaissance, il s'agit d'un cas unique de RTT typique relié à une anomalie de FOXG1. Ces données confirment l'hétérogénéité génétique du syndrome de Rett. Elle précise également le spectre des phénotypes associés à des mutations de FOXG1, un nouveau gène à ne pas négliger dans les formes typiques et peut-être moins sévères (forme fruste, régression tardive ?) de RTT.

Mots-clés : RETT, FOXG1.

EP527. BILAN LYONNAIS DE L'ÉTUDE MOLÉCULAIRE DU GËNE CDKL 5

N. Boutry-Kryza (1), D. Ville (2), F. Dalmon (3), C. Vercherat (4), A. Calender (1), V. Desportes (2), G. Lesca (1)

(1) Laboratoire de Génétique Moléculaire, Hôpital Édouard Herriot, Hospices Civils de Lyon, France; (2) Service de Neuropédiatrie, Hopital Femme-Mère-Enfant, Hospices civils de Lyon, France; (3) Service de Pédiatrie, CH Chambéry. France; (4) INSERM, U865, Lyon, France Contact : nadia.kryza@chu-lyon.fr

Des mutations du gène CDKL5, situé en Xp22, ont été mises en évidence depuis 2004 chez des sujets atteints d'une forme atypique de syndrome de Rett avec début précoce des crises d'épilepsies. Ce gène a ensuite été impliqué de façon plus générale chez des sujets présentant des spasmes infantiles ou syndrome de West. Il code pour une protéine aux fonctions encore mal élucidées mais qui semblerait interagir avec la protéine Mecp2. Le diagnostic moléculaire a débuté en 2008 au laboratoire de génétique de Lyon. 31 patients ont été testés à ce jour : 27 filles et 4 garçons. Sur le plan clinique, 13 enfants ont été adressés pour syndrome de Rett atypique et ont déjà bénéficié d'une analyse du gène MECP2 qui s'est avérée négative et 18 enfants ont été adressés pour épilepsie précoce avec spasmes. Le diagnostic moléculaire repose sur le séquençage des régions codantes du gène: l'exon 1 étant transcrit mais non traduit, 20 exons ainsi que les jonctions intron-exon sont analysés. 4 mutations pathogènes ont été mises en évidence : La mutation p.Tyr594X, mise en évidence chez une fille de 2 ans présentant un syndrome de West typique, a déjà été rapportée dans la littérature chez un patient avec un tableau clinique proche. La mutation p.Tyr177Cys, est retrouvée chez une fille de 10 ans présentant un syndrome de Rett atypique avec des antécédents personnels marqués par une encéphalopathie épileptique précoce. Cette mutation n'est pas répertoriée à ce jour dans la littérature mais est située dans une zone fonctionnelle importante de la protéine, le domaine sérine/thréonine protéine kinase. De plus, d'autres mutations faux-sens situées à proximité sont associées à la maladie (acides aminés 175,178 et 180). La mutation p.Gly264X a été mise en évidence à l'état de mosaïque chez une fille de 2 ans présentant un phénotype atypique. La mosaïque a été confirmée par la collection des 2 populations après passage sur dHPLC et séquençage. La patiente présente sur le plan clinique un syndrome de Rett atypique sans convulsion 
à ce jour. La présence de cette mutation à l'état de mosaïque expliquerait le phénotype atypique. Enfin, la mutation du site d'épissage c,-164G>A est présente à l'état hémizygote chez un garçon atteint d'une encéphalopathie épileptique précoce avec spasmes. Sa pathogénicité a été confirmée par l'analyse de l'ADN complémentaire du gène montrant la production de 3 transcrits anormaux. Ce bilan à 2 ans montre un taux de détection de mutations d'environ $13 \%$ chez nos patients. Ces résultats confirment la nécessité de rechercher des mutations du gène CDKL 5 pour les encéphalopathies épileptiques précoces avec spasmes chez les filles mais également chez les garçons. La présence de mosaïque est également à rechercher et peut justifier l'analyse d'un autre tissu en cas d'analyse négative chez un sujet avec phénotype évocateur. L'analyse de ce gène se poursuivra prochainement par la mise en place de la recherche de grands réarrangements du gène CDKL5.

Mots-clés : CDKL5, spasmes infantiles.

שP528. L'HAPLOINSUFFISANCE DU GÈNE SIMI EST RESPONSABLE D'OBÉSITÉ ET D'UN NOUVEAU SYNDROME DE TYPE PRADER-WILLI-LIKE

F. Stutzmann (1), M. Ghoussaini (1, 2), C. Couturier (1), S. Bouquillon (3, 4), M. Marchand (1), V. Vatin (1), J. Andrieux (4), B. Delobel (3), N. Calmels (5), J.L. Mandel (5, 6), B. Balkau (7), F. Horber (8), L.F. Van Gall (9), W. Van Hul (10), F. Pattou (11), A.P. Goldstone (12), D.J. Driscoll (13), M. Whitelaw (14), D. Meyre (1), P. Froguel (1, 15)

(1) CNRS-UMR-8090, Institut de Biologie de Lille, Université Lille2, Institut Pasteur, Lille, France; (2) Cancer Research UK, Department of Oncology, University of Cambridge, Strangeways Research Laboratory, Cambridge, United Kingdom ; (3) Centre de Génétique Chromosomique, Hôpital St-Vincent-de-Paul, GHICL, Lille, France; (4) Laboratoire de Génétique Médicale, Hôpital Jeanne-de-Flandre, CHRU de Lille, France ; (5) Laboratoire de diagnostic génétique, CHRU Strasbourg- Nouvel Hôpital Civil, Strasbourg, France ; (6) Institut de Génétique et de Biologie Moléculaire et Cellulaire, Inserm U596, CNRS, UMR7104; Université Louis Pasteur, Strasbourg, France : (7) INSERM U780, Université ParisSud, Orsay, France; (8) Klinik Lindberg, Winterthur, Switzerland; (9) Department of Endocrinology, Diabetology and Metabolism, Antwerp University Hospital, Antwerp, Belgium ; (10) Department of Medical Genetics, University of Antwerp and Antwerp University Hospital, Antwerp, Belgium; (11) INSERM U859, CHU Lille, Lille, France ; (12) MRC Clinical Sciences Centre and Endocrine Unit, Department of Investigative Medicine, Hammersmith Hospital, Imperial College, London, United Kingdom; (13) University of Florida, Gainesville, Florida; (14) Discipline of Biochemistry, School of Molecular \& Biomedical Science - Centre for the Molecular Genetics of Development University of Adelaide, Adelaide, South Australia, Australia ; (15) Section of Genomic Medicine, Imperial College London, Burlington-Danes Building, Hammersmith Hospital, Du Cane Road, London, United Kingdom

Contact : philippe.froguel@good.ibl.fr

Le syndrome de Prader-Willi est caractérisé par une obésité hyperphagique, un hypogonadisme, un retard de développement et des caractéristiques faciales. Des anomalies chromosomiques au 6q16, incluant SIM1, sont responsables d'un syndrome Prader-Willi-like (6q-PWLS). L'homologue du gène Single Minded de Drosophila est un facteur de transcription impliqué dans le développement des lignées neuroendocrines. Or l'unique cas de translocation équilibrée affectant SIM1 a été identifié chez une fillette obèse ne présentant pas de trouble cognitif. Dans cette étude, nous avons donc recherché la contribution de la perte de fonction d'un allèle de SIM1, à la fois à l'obésité monogénique et syndromique chez l'homme. SIM1 a été séquencé chez 43 enfants obèses américains et 288 enfants obèses français avec PWLS, sans anomalie chromosomique aux locus $15 q$ et $6 \mathrm{q}$ détectée par caryotype ni anomalie de méthylation au $15 \mathrm{q}$, ainsi que chez 799 sujets présentant une obésité précoce non-syndromique, sévère ou familiale, venant des États-Unis, de France, de Suisse et de Belgique. La recherche de mutations incluait aussi 835 individus contrôles minces, sélectionnés pour correspondre aux sujets obèses en âge, sexe et ethnie. Des microdélétions de SIM1 ont été recherchées par PCR quantitative et confirmées et limitées par puces CGH chez les 288 enfants français PWLS. Au total, 19 variants non synonymes de SIM1 ont été identifiés chez 22 sujets : 6 ont été observés chez les sujets obèses (fréquence $=0,8 \%$ ), 8 chez les enfants avec PWLS $(2,4 \%)$ et 8 chez les contrôles $(1,0 \%)$. La caractérisation fonctionnelle des variants est en cours. Des résultats préliminaires d'expériences de gène rapporteur ont montré que cinq variants, identifiés chez 4 enfants avec PWLS et un sujet obèse, conduisent à une diminution significative de l'activité de SIM1 (activité résiduelle: $20,4-53,5 \%, p<0,05)$. Ces variations coségrègent avec obésité et retard mental dans les familles des propositus. Les phénotypes observés chez les patients obèses et leurs apparentés porteurs de ces mutations pathogéniques sont : obésité, retard mental, visage rond, petit nez, arête nasale déprimée, philtrum proéminent et fente palpébrale étroite mais une absence d'hypotonie néonatale. Contrairement au syndrome PW du 15q, l'hypogonadisme est anecdotique. Des troubles du comportement et un retard de développement ont été observés chez la moitié des sujets. Une délétion au locus $6 q 16$ de $3,5 \mathrm{Mb}$ et $4 \mathrm{Mb}$ a été identifiée chez deux enfants supplémentaires présentant des caractéristiques syndromiques similaires. Cette étude montre que l'haploinsuffisance de SIM1 est responsable d'une forme syndromique de type PWL non complètement pénétrante. Le spectre clinique spécifique de ce syndrome devrait inciter au diagnostique moléculaire. Enfin, la recherche de mutations de SIMI présente un intérêt clinique pour certaines formes d'obésité infantile associée avec un retard mental, en complément d'investigations cytogénétiques.

Mots-clés : SIM1, syndrome, obesity.

IP529. L'HÉTÉROPLASIE OSSEUSE PROGRESSIVE ET LA PSEUDOHYPOPARATHYROIDIE : DEUX PHÉNOTYPES POUR LES MÊMES MUTATIONS INACTIVATRICES DU GENE GNAS, UN PHÉNOMÈNE D'EMPREINTE PARENTALE

M. Lebrun, N. Richard, G. Abeguilé, N. Coudray, M.L. Kottler

Département de Génétique et Reproduction, Laboratoire de biologie moléculaire, CHU-caen, France

Contact : kottler-ml@chu-caen.fr

L'hétéroplasie osseuse progressive ( $\mathrm{POH}$ ) est une maladie rare de formation osseuse ectopique. $\mathrm{La} \mathrm{POH}$ est due à des mutations hétérozygotes inactivatrices du gène GNAS, aussi connu pour provoquer la pseudohypoparathyroïdie de type la (PHPla), quand elle est transmise par la mère et la pseudopseudohypoparathyroïdie (PPHP), quand elle est transmise par le père. Des études familiales ont également associé la POH à une transmission paternelle. L'objectif de cette étude est de caractériser l'origine parentale de l'allèle muté dans des cas « de novo» de $\mathrm{POH}$, et d'établir des corrélations phénotype/génotype selon la transmission maternelle ou paternelle. Nous avons apparié 10 cas de $\mathrm{POH}$, tous sporadiques sauf un, avec des cas de PHPla portant la même mutation GNAS. Nous décrivons leur phénotype et leur profil hormonal et d'activité Gsalpha. L'origine parentale de l'allèle muté a été étudiée en utilisant des polymorphismes intragéniques informatifs et le sous-clonage des produits de PCR. Nous avons clairement démontré l'origine paternelle des mutations de GNAS chez 8 patients diagnostiqués avec une $\mathrm{POH}$. Dans un cas, le constat d'une mutation d'origine maternelle nous a permis de faire le diagnostic de «POH-like PHPla». Les corrélations génotype/phénotype suggèrent l'absence de corrélation directe entre le processus d'ossification et l'origine parentale, étant donnée la grande variabilité dans les ossifications hétérotopiques. Un retard croissance intra utérin sévère a été mis en évidence dans les mutations d'origine paternelle. L'hétérogénéité clinique rend le conseil génétique très délicat, en particulier quand il s'agit de transmission paternelle, qui peut conduire à la fois à une expression modérée de PPHP ou sévère de $\mathrm{POH}$.

Mots-clés: gène GNAS, hétéroplasie osseuse progressive, empreinte parentale.

IP530. SYNDROME MACS PAR MUTATION DU GÈNE RIN2 : DESCRIPTION DE LA SECONDE FAMILLE ET NOUVELLES DONNÉES PHÉNOTYPIQUE

F. Malfait (1), A. De Paepe (1), G. Mortier (1), A. Benmansour (2), A. Verloes (3)

(1) Département de génétique, Université de Gand, Belgique ; (2) Pédiatre, Oran, Algérie; (3) Département de Génétique, APHP - Hôpital Robert Debré, Paris, France

Contact : alain.verloes@rdb.aphp.fr

Nous rapportons le phénotype et l'histoire naturelle de 3 enfants issus d'un couple consanguin d'origine algérienne, présentant un tableau clinique complexe et évolutif. Les éléments principaux sont une scoliose progressive sévère, une ostéoporose, une hyperlaxité très marquée des articulations des mains et des chevilles, une atrophie cutanée généralisée associée à un aspect de cutis laxa, sans anomalies de la cicatrisation, une macrocéphalie, une dysmorphie faciale, une hypertrophie des gencives et des tissus mous péribuccaux, une hypotrichose, des anomalies rétiniennes et une craniosténose. Le screening du gène RIN2 (20p11.21-p11.23) a montré la présence d'une délétion homozygote de 2 nucléotides, avec décalage du cadre de lecture dans l'exon 8 (c.1914_1915delGC, p Glu638AspfsX9). Cette mutation confirme l'observation par Basel-Vanagaite (Am J Hum Genet. 2009 Aug : 85(2): 254-63) d'une grande famille palestinienne présentant un phénotype similaire (pour lequel l'acronyme MACS - macrocephaly, 
alopecia, cutis laxa, and scoliosis - a été proposé). Dans cette famille, une délétion homozygote dans l'exon 7 de RIN2a été démontrée. RIN2 est une protein ubiquitaire impliquée dans le traffic des vésicules endocytiques. Notre observation constitue la $2^{c}$ observation de syndrome MACS. elle confirme l'existence de cette entité et la spécificité de son phénotype clinique.

Mots-clés : Ehlers-Danlos, RIN2.

- 531. FORTE MYOPIE AVEC ANTÉCÉDENTS D'OMPHALOCÈLE OPÉRÉE : LE SYNDROME DE DONNAI-BARROW

P. Bitoun (1A), E. Pipiras (1B), B. Benzacken (1B), A. Delahaye (1B)

(IA) Génétique Médicale, CHU Jean Verdier Paris XIII, Bondy, France ; (1B) Histo-Embryo-Cytogénétique, CHU Jean Verdier Paris XIII, Bondy, France

Contact : bitoun@gmail.com

Le syndrome de Donnai-Barrow associe omphalocèle, myopie forte, agénésie du corps calleux cardiopathie, surdité, un retard de développement inconstant et une néphropathie. Une vingtaine de cas ont été décrits depuis 1993. Patients et Méthodes: Les auteurs présentent une famille consanguine dont le fils ainé présente une omphalocèle de découverte anténatale et une forte myopie avec hypertélorisme et dont la sœur présente les mêmes symptomes. Les patients sont explorés par IRM, bilan auditif; rénal et cardiaque. Résultats: L'ainé de 6 ans présente un développement normal ; il est issu d'une grossesse compliquée d' omphalocèle ayant nécessité une césarienne à 38 semaines et une croissance anténatale normale ; L'omphalocèle est opérée à $\mathrm{J} 2$ et une CIA est identifiée en échographie qui se résoudra spontanemment. Il présente un hypertélorisme et une forte myopie bilatérale à 28 dioptries et une acuité visuelle de loin à $2 / 10^{\epsilon}$ et $\mathrm{P} 2$ avec une longueur axiale à $35,8 \mathrm{~mm}$. Sa scuur de 4 ans présente alors la même omphalocèle opérée en périnatal, une CIA avec CIV résolutives et une forte myopie à 18 et 17 dioptries et un prognatisme. L'IRM cérébrale du garçon était normale. Une hypoacousie modérée bilatérale est alors identifiée à $55 \mathrm{db}$ et une protéinurie est notée avec une microglobulinurie à 145000 microg/l $(\mathrm{N}<205$ microg/l) avec créatininémie, Calcémie, albuminémie et Vitamine A normales. Il présente à l'age de 7 ans un décollement de rétine opéré. La sœur présente aussi une hypoacousie à $40 \mathrm{db}$, et une protéinurie. Ils présentent tous deux une intelligence normale et les parents sont en bonne santé. L'association omphalocèle, forte myopie, cardiopathie, hypoacousie et protéinurie constitue le syndrome de Donnai Barrow lié aux mutations de LRP2 codant pour la mégaline récepteur multiligand énorme gène comme son nom l'indique. Ce diagnostic doit être évoqué devant cette association très caractéristique forte myopie et omphalocèle.

Mots-clés : myopie, omphalocèle, syndrome de Donnai-Barrow.

EP532. RECHERCHE D'UN LOCUS GÉNOMIQUE IMPLIQUÉ DANS LES SYNDROMES TRICHO-HÉPATO-ENTÉRIQUES

A. Fabre (1, 2), B. Roquelaure (1), C. Lacoste (2), J. Martinez (3), N. Peretti (4), N. André (5), A. Breton (6), C.M. Martinez-Vinçon (7), N. Levy $(2,9)$, O. Goulet (8), C. Badens $(2,9)$

(1) Service de Pédiatrie Multidisciplinaire, Hôpital d' enfants de la Timone, Marseille, France; (2) Laboratoire de Génétique Moléculaire, Département de Génétique, Hôpital d'enfants de la Timone, Marseille, France; (3) Laboratoire de cytogénétique, Département de Génétique, Hôpital d'enfants de la Timone, Marseille, France ; (4) Service de Nutrition pédiatrique, Hôpital Femme Mère Enfant de Lyon, France; (5) Service de Pédiatrie Oncologique, Hôpital d'enfants de la Timone, Marseille, France; (6) Service d'Hépatologie et Maladies Métaboliques, Hôpital d'enfants de Toulouse, France ; (7) Service de Gastro-entérologie, Hôpital Robert Debré, Paris, France ; (8) Service de Gastro-Entérologie, Hôpital Necker-Enfants Malades, Paris, France; (9) Unité Inserm U910, Marseille, France

Contact : alexandre.fabre@ap-hm.fr

Les syndromes Tricho-Hépato-Entériques (THE) ou Diarrhées Graves Rebelles Syndromiques associent une diarrhée grave rebelle chronique, une dysmorphie, des anomalies immunitaires, un retard de croissance intra-utérin et des anomalies des cheveux (cheveux laineux avec trichorrhexis nodosa). Une dizaine de patients atteints de ce syndrome a été diagnostiquée en France : ils présentent un phénotype très homogène et la moitié d'entre eux sont issus d'unions consanguines. Une collection d'échantillons des patients et de leurs parents a été réalisée en 2006 et déclarée au CRB de la Timone à Marseille. En vue de caractériser une localisation génomique impliquée dans la survenue de ce syndrome, nous avons, dans un premier temps, testé une approche gènes candidats. Au cours d'une étude moléculaire, sept gènes candidats fonction- apparentés chez l'homme ou chez l'animal, ont été exclus (Fabre et al., 2009). Deux autres approches ont alors été mises en cuvre: - une recherche pangénomique d'insertions/duplications par technique de CGH array, qui a mis en évidence chez un des patients une délétion en 15q11.2, confirmé en PCR quantitative. Les délétions de ce locus sont associées dans la littérature, à des syndromes autistiques, syndrome que l'on retrouve chez ce patient mais qui est probablement associé de façon fortuite au syndrome THE. Aucune autre anomalie potentiellement pathogène n'a été détectée. -une étude de liaison pangénomique par analyse de SNP. L'analyse de liaison directe n'a pas retrouvé de région pouvant être incriminée. En revanche, la recherche de régions d'homozygotie a permis de caractériser une zone de $5.1 \mathrm{Mb}$ commune à 2 des familles consanguines. Cette région contient 35 gènes que nous nous proposons de séquencer afin de mettre en évidence un éventuel défaut moléculaire. L'identification du ou des gènes altérés dans cette pathologie conduira à l'exploration fonctionnelle des protéines concernées. En particulier, le profil d'expression tissulaire pourra être exploré ainsi que la localisation et la fonction cellulaire. À terme, l'identification de la cause génétique responsable de cette pathologie permettra de proposer aux familles d'enfants atteints un diagnostic de certitude ainsi que d'améliorer les connaissances de la physiopathologie des diarrhées intraitables.

Mots-clés : syndrome tricho-hépato-enterique.

\section{IP533. STORMORKEN SYNDROME : CONFIRMATORY REPORT IN A FRENCH FAMILY}

G. Morin (1), B. Roméo (2), R. Cévallos (3), B. Demeer (1), A.G. Le Moing (1), J.C. Capiod (4), E. Bourges-Petit (5), H. Sevestre (6), M. Mathieu (7)

(1) Clinical Genetics Unit, Amiens University Hospital, Amiens, France; (2) Pediatric Pneumology, Amiens University Hospital, Amiens, France ; (3) Internal Medecine, Amiens University Hospital, Amiens, France ; (4) Hematology Laboratory, Amiens University Hospital, Amiens, France ; (5) Pediatric Cardiology, Amiens University Hospital, Amiens, France; (6) Pathology Laboratory, Amiens University Hospital, Amiens, France Contact : morin.gilles@chu-amiens.fr

In 1985, Helge Stormorken reported a new syndrome associating thrombocytopathia, muscle fatigue, asplenia, miosis, migraine, dyslexia and ichthyosis. This affection segregated in a Norvegian family with an apparent autosomal dominant transmission on 4 generations. The physical characteristics were documented in a teen-aged boy and his mother : small stature, deep set eyes, high and arched forehead, permanent miosis without efficiency of mydriatic drugs and decreased darkness vision. A bleeding tendency was demonstrated, including hematomas, frequent nose bleedings but no major hemorrhagic accident. Hematologic investigations exhibited consistently prolonged bleeding time, normal coagulation, presence of giant platelets and various abnormal forms of red cells with Howell-Jolly bodies in the peripheral blood. The spleen was absent in both patients explaining the post-splenectomy aspect of the red cells. The muscular defect consisted in limitation during physical exercise and an abnormally increased duration of muscle response. Ichthyosis was mainly located in the extremities. Both patients suffered from headaches with photophobia. Dyslexia was early diagnosed for the affected son, responsible of learning disability, but less severe for his mother. From that time, this disease was only reported in a Japanese mother and daughter, who both presented muscle weakness, moderate increasing of creatine kinase rates and thrombocytopenia. Muscle biopsy showed fibre necrosis and regeneration, variation in fibre size, and tubular aggregates in approximately $5 \%$ of the fibres. We report the observation of a young boy and his father. At 17 days of life the baby presented an urticaria-like eruption on the face and the lower limbs. Blood count revealed thrombopenia $\left(48000 / \mathrm{mm}^{3}\right)$ but the myelogram was normal. During the hospitalization, he presented an episode of supraventricular tachycardia requiring a treatment with acebutolol and amiodarone. The father had small stature $(157 \mathrm{~cm})$, high pitched voice, chronic eruption of the arms and shoulders partially regressive after anti-mycosic treatment, and a severe but reactive miosis responsible of impairment of darkness vision. $\mathrm{He}$ also presented asplenia and moderate thrombocytopenia $\left(97000 / \mathrm{mm}^{3}\right)$. At the age of 2 years and half, a less severe miosis became more apparent in the son. Peripheral blood examination revealed anisocytosis, the presence of macrothrombocytes, Howell-Jolly bodies and prolonged bleeding time.

Mots-clés : Stormorken syndrome, congenital miosis, thrombocytopathia.

- 5 534. NOUVELLE MUTATION NON SENS DU GÈNE GPC3 CHEZ UN PATIENT MAROCAIN ATTEINT DU SYNDROME DE SIMPSON-GOLABI-BEHMEL

I. Ratbi (1, 2), S. Chafai Elalaoui (1), M.P. Moizard (3), M. Raynaud (3), A. Sefiani $(1,2)$

(1) Département de Génétique Médicale, Institut National d'Hygiène, Rabat, Maroc ; (2) Centre de Génomique Humaine, Faculté de Médecine 
et de Pharmacie de Rabat, Université Mohammed V Souissi, Rabat, Maroc; (3) Service de Génétique, Hôpital Bretonneau, Tours, France Contact : ilhamratbi@yahoo.fr

Le syndrome de Simpson-Golabi-Behmel (SGBS) se caractérise par une avance de croissance globale pré et post-natale, une dysmorphie faciale et des malformations viscérales et squelettiques. Plus d'une centaine de patients atteints de SGBS ont été rapportés à ce jour. Le SGBS est de transmission récessive liée à l'X. Un gène principal, Glypican 3 (GPC3) a été identifié en Xq26. Différents types de mutations ont été rapportées à ce jour. Nous avons vu en consultation de génétique médicale un garçon âgé de 5 ans, issu de parents consanguins, deuxième d'une fratrie de deux, adressé pour syndrome dysmorphique. Il est né par voie basse au terme d'une grossesse suivie. Il a eu un retard des acquisitions psychomotrices, et présente un retard mental. À l'examen clinique, on note une avance staturo-pondérale à $+2 \mathrm{DS}$, une dysmorphie faciale faite d'une racine du nez effacée, des narines antéversées, une macrosotmie des oreilles décollées et des caries dentaires. Il présente par ailleurs des mamelons surnuméraires, une hexadactylie post axiale au niveau de la main gauche et une cryptorchidie gauche. Le bilan paraclinique a montré une synostose costale et une hypothyroïdie. Enfin, l'échographie abdomino-pelvienne, le bilan squelettique, l'échocardiographie et l'examen ophtalmologique étaient normaux. Devant l'association de l'avance staturo-pondérale et la dysmorphie faciale particulière, une étude moléculaire du gène GPC3 a été réalisée, et a montré que le patient est porteur d'une mutation non sens encore non rapportée (c.271C>T; p.Gln91X) localisée au niveau de l'exon 2. Par ailleurs, la mère n'est pas porteuse de la mutation retrouvée chez son fils.

Mots-clés : syndrome de Simpson-Golabi-Behmel, gène GPC3, mutation ponctuelle.

IP535. L'ANALYSE PAR LES PUCES SNP, DE CAS SPORADIQUES DANS DES FAMILLES CONSANGUINES, PERMET D'ORIENTER LE DIAGNOSTIC MOLÉCULAIRE DE PATHOLOGIES AUTOSOMIQUES RÉCESSIVES HÉTÉROGÈNES

C. Gasnier (1), N. Dondaine (1), M.C. Vincent (1, 2), E. Schaefer (3), M. Cossée $(1,2)$, C. Lagier-Tourenne $(1,4)$, H. Dollfus $(3,2)$, C. Tranchant (5), P. Charles (6), J. Amiel (7), C. Antignac (7), I. Vuillaume (8), C. Thibault (4), M. Koenig $(1,4)$, J.L. Mandel $(1,4)$

(1) Laboratoire de Diagnostic Génétique, Hôpitaux Universitaires de Strasbourg, Strasbourg, France ; (2) Laboratoire de Génétique Médicale, EA3949, Faculté de Médecine, Hôpitaux Universitaires de Strasbourg, Strasbourg, France; (3) Service de Génétique Médicale, Hôpitaux Universitaires de Strasbourg, Strasbourg, France; (4) IGBMC (CNRS/ INSERM/ULP), Illkirch, France ; (5) Service de Neurologie, Hôpital Civil, Hôpitaux Universitaires de Strasbourg, Strasbourg, France; (6) Consultation de Génétique, Groupe Hospitalier Pitié-Salpêtrière, AP-HP, Paris, France; (7) Département de Génétique, Groupe hospitalier universitaire Necker Enfants malades, Paris, France; (8) Centre de Biologie-Pathologie, CHRU Lille, France

Contact : claire.gasnier@chru-strasbourg.fr

Le diagnostic moléculaire des maladies rares à transmission autosomique récessive présentant une grande hétérogénéité génétique représente un véritable défi car les données cliniques ne permettent pas, dans la plus part des cas, de cibler un gène défectueux en particulier. Dans de telles familles la consanguinité est fréquente. À l'échelle du génome entier l'analyse de puces SNP permet, par la recherche de régions homozygoties chez ces patients, la sélection d'un ou de quelques gènes candidats parmi lesquels rechercher des mutations. Voici 7 cas de ce type dont 5 sporadiques, pour lesquels cette approche à permis de déterminer la mutation du gène responsable de la maladie. Par exemple, l'étude d'une forme sporadique de myopathie a permis de mettre en évidence une mutation de TRIM32, également, l'étude d'une forme familiale d'ataxie a mis en évidence une mutation sur AOA1. La cartographie des régions d'homozygotie, par l'utilisation des micro-puces $50 \mathrm{~K}$ (Affymetrix), a été effectuée uniquement chez les patients et nous a permis d'identifier la mutation causale dans une proportion importante de cas sporadiques atteints de différentes maladies neuromusculaires ou neurodégénératives. Cette approche rapide et économique est particulièrement utile pour des maladies ayant une grande hétérogénéité génétique comme le syndrome de Bardet Biedl (14 gènes publiés à ce jour), la dystrophie musculaire des ceintures ou des ataxies sporadiques. En effet, elle permet de sélectionner seulement un ou deux gènes à séquencer et ainsi d'identifier la mutation privée. Dans certains cas, l'analyse de puces SNP peut mettre en évidence une consanguinité qui était inconnue ou refusée par la famille.

Mots-clés: puces SNP, autosomiques récessives hétérogènes, cas sporadiques.

口P536. HÉMORRAGIE TÉLANGIECTASIQUE HÉRÉDITAIRE : DEUX EFFETS FONDATEURS DANS LE GENE ACVRLI EN RÉGION POITOU-CHARENTES

D. Haye (1), S. Patri (1, 2), A.M. Delvaux (1, 2), J.Y. Lehy (3), A. Kitzis $(1,2)$, B. Gilbert-Dussardier (1)

(I) Service de Génétique, Centre de Compétence Maladie de Rendu Osler, CHU de Poitiers, Poitiers, France ; (2) CNRS UMR6187, Institut de Physiologie et de Biologie Cellulaires, Université de Poitiers, Poitiers, France ; (3) Médecin Généraliste, Arçais, France

Contact :dhaye@hotmail.fr

L'Hémorragie Télangiectasique Héréditaire (HTH) ou Maladie de RenduOsler est une maladie autosomique dominante caractérisée par des malformations artério-veineuses pouvant affecter plusieurs organes. Des mutations causales sont décrites dans deux gènes (ENG et ACVRL1) codant pour des récepteurs impliqués dans la voie de signalisation du TGFbeta. La prévalence de la maladie est estimée à plus de $1 / 10000$ en France, avec d'importantes disparités géographiques. En 1989, une étude épidémiologique a estimé la prévalence à $1 / 4287$ dans le département des DeuxSèvres, avec une concentration plus importante dans les arrondissements de Parthenay (1/1 193) et de Bressuire (1/2 430), L'étude moléculaire des gènes ENG et ACVRL1 réalisée chez 110 patients originaires de la région Poitou-Charentes, a mis en évidence l'existence de deux mutations majoritaires dans le gène ACVRL1. Une mutation faux-sens, c.1121G>A (exon 8 - p.Arg374Gln) et une mutation d'épissage, c. 1048+1G>A (intron 7) touchant respectivement 44 patients répartis dans 8 familles et 30 patients répartis dans 11 familles. Nous montrons par analyse de marqueurs microsatellites situés dans une région de 10 mégabases centrée sur le gène ACVRL1, un haplotype spécifique pour chacune des deux mutations régionales, suggérant l'existence de deux effets fondateurs. L'étude de ces 19 familles montre deux foyers géographiques de la maladie dans le Nord des Deux-Sèvres, l'un à proximité de la sous-préfecture de Parthenay, le second à proximité de la sous-préfecture de Bressuire. Par ailleurs, la description clinique de la maladie pour chaque patient porteur de l'une des mutations confirme une grande variabilité d'expression déjà connue dans la maladie. La mise en évidence d'un effet fondateur commun pour un nombre important de patients est d'intérêt en prévision d'études cherchant à identifier des gènes modificateurs à l'origine de la variabilité d'expression.

Mots-clés: hémorragie télangiectasique héréditaire, Poitou-Charentes, effets fondateurs.

- 5 537. CORRÉLATION GÉNOTYPE-PHÉNOTYPE DANS LE SYNDROME D'EHLERS-DANLOS VASCULAIRE : ANALYSE DE 86 CAS

A.L. Fauret $(1,2)$, L. Bal $(2,3)$, B. Ranque $(2,4)$, C. Travers (1), J. Perdu $(1,2)$, M. Frank (3), E. Messas (2, 3), D.P. Germain (5), H. Plauchu (6), J. Emmerich (2, 3), J.N. Fiessinger (2, 3), X. Jeunemaitre $(1,2)$

(1) AP-HP, Hôpital Européen Georges-Pompidou, Département de Génétique et Centre de Référence des Maladies Vasculaires Rares, Paris, France: (2) Université Paris Descartes, Faculté de Médecine, Paris, France; (3) AP-HP, Hôpital Européen Georges-Pompidou, Service de Médecine Vasculaire et Centre de Référence des Maladies Vasculaires Rares, Paris, France ; (4) AP- HP, Hôpital Européen Georges-Pompidou, Service de Médecine Interne, Paris, France ; (5) AP- HP, Hôpital Raymond-Poincaré, Service de Génétique Médicale, Garches, France; (6) Hôpital de l'Hôtel-Dieu, Service de Génétique, Lyon, France.

Contact : anne-laure.fauret@egp.aphp.fr

Background. The vascular Ehlers-Danlos syndrome (vEDS) is a rare autosomal dominant inherited disorder, caused by mutations in the COL $3 \mathrm{~A} 1$ gene, which results in the synthesis of a defective pro-alphaI chains of type III procollagen. Affected patients are at risk for arterial, bowel, and uterine rupture. The mechanisms by which mutations in the COL $3 \mathrm{~A} 1$ gene produce diseases are poorly understood. No correlations between the nature or location of the mutation and the type or frequency of major complications have been described. Methods. We reviewed the clinical and family histories, medical and surgical complications of all patients with causative COL3A1 mutation in our Centre de Maladies Vasculaires Rares since 2001. The search for mutation was performed by direct sequencing from cDNA obtained from cultured dermal fibroblasts and/or from peripheral genomic DNA. Results. 86 vEDS patients with causative mutation were identified, including 66 probands and 20 relatives. One third of patients had a family history of sudden death. There was no statistical difference between males $(n=32)$ and females $(n=54)$, except for a more frequent presentation with Madone's features in females $(89 \%$ vs. $65 \%, p=0.02$ ). The median age at the first major complication was 25 years for probands and 35 years for relatives, whereas the median age at diagnosis was younger in relatives 
( 21 years) than in probands ( 32 years, $\mathrm{p}<0.02$ ) as a consequence of the availability of the molecular diagnosis. The first major complications were vascular $(52.5 \%)$, digestive $(25 \%)$ and obstetrical $(7.5 \%)$. Median age at the first complication was lower for probands with a digestive first complication ( 23 years) than for patients with vascular or obstetrical first complication ( 30 years, $p=0.01$ ). Moreover, their second major complication was more frequently digestive as compared to probands with a vascular first complication $(35.3 \%$ vs $7.5 \% \mathrm{p}=0.03)$. Sixty different mutations were found: 37 missense $(61.6 \%)$ affecting a Gly residue in the G-X-Y triplets in the triple helix, 15 splice mutations $(25 \%), 3$ indel mutations, 4 other missense mutations including 2 located in the $\mathrm{C}$-term and 1 nonsens in the C-term (Y178X). There was no clinical difference between the 54 patients with Gly missense mutations and the 24 patients with splice and indel mutations but a remarkably less typical morphotype $(p<0.001)$ and less severe course in the 8 subjects with other missense or C-term nonsense mutations. In particular, the age of the first major complication was significantly higher in this latter group (44 [35-46] years) compared to patients with a Gly missense mutation (28 [21-37], $\mathrm{p}=0.01)$. Conclusion. This study suggests for the first time genotype-phenotype relationships that may affect the genetic counseling and the indication of a genetic test on COL $3 \mathrm{~A} 1$ gene. The diagnosis of vEDS should be considered in subjects with unexplained arterial rupture, even in absence of a classical morphotype.

P538. RECHERCHE DE MUTATIONS DANS LES GENNES ACVRL1 ET ENDOGLIN IMPLIQUÉS DANS LA MALADIE DE RENDU-OSLER SUR UNE POPULATION DE 150 PATIENTS ET APPARENTÉS

S. Patri, D. Haye, A.M. Delvaux, B. Gilbert-Dussardier, A. Kitzis Service de Génétique, CHU de Poitiers, Poitiers, France

\section{Contact : s.patri@chu-poitiers.fr}

La maladie de Rendu-Osler ou Hémorragie Télangiectasique Héréditaire (HHT) est une dysplasie vasculaire constitutionnelle d'origine génétique. Deux gènes sont principalement impliqués dans cette maladie : ACVRL1 (HHT2) et Endogline (HHT1). Ces deux gènes interviennent dans la transduction du signal TGFbeta. HHT est une maladie à transmission autosomique dominante dont la prévalence est de l'ordre de $1 / 10000$ en France, avec de grandes variations en fonction des régions. L'expression de la maladie est aussi extrêmement variable avec une très importante disparité des manifestations clinique allant de la présence d'épistaxis et/ou de télangiectasies jusqu'à des malformations artério-veineuses pulmonaires, neurologiques ou hépatiques, jusqu'au sein d'une même famille. Le diagnostic est clinique mais l'identification de la mutation causale du proposant est primordiale pour pouvoir proposer dans la famille le dépistage des sujets asymptomatiques ou pauci-symptomatiques qui en font la demande. Nous avons étudié $150 \mathrm{ADN}$ de patients atteints de la maladie de Rendu-Osler ou d'apparentés. Pour ceci nous avons développé une technique d'étude des gènes ACVRL1 et Endogline par l'analyse des courbes de fusion haute résolution (HRM) sur un appareil 7500-Fast (Applied Biosystem). La mise au point a été faite de telle façon que toutes les amplifications soient réalisées dans les mêmes conditions. Le gène ACVRL1 est composé de 9 exons codants, 7 sont étudiés par la technique HRM en 12 amplicons ce qui permet de génotyper 5 ADN sur une plaque. Les 2 exons restants sont étudiés par séquençage direct, l'un à cause d'un polymorphisme extrêmement fréquent et l'autre parce qu'il n'a pas été possible de l'amplifier dans les mêmes conditions que les autres. Le gène Endogline est composé de 14 exons, tous étudiés par la technique HRM en 14 amplicons. Ainsi 2 plaques suffisent pour étudier 9 patients. Les éventuelles délétions chez les cas index pour lesquels nous ne trouvons aucune mutation ponctuelle sont recherchées par la technique MLPA (P193-B2, MRC-Holland) puis vérifiées par PCR multiplex semiquantitative sur DHPLC. Dans la population étudiée à Poitiers nous trouvons pour le gène ACVRL1 : 1 délétion complète du gène et 18 variations potentiellement causales dont 8 ne sont pas décrites dans la littérature ( 4 faux sens, 1 non sens, 1 délétion de 1 base, 1 délétion de 3 bases et 1 insertion-délétion complexe). Pour le gène Endogline notre population compte 10 variations potentiellement causales dont 2 ne sont pas décrites ( 1 non sens et 1 faux sens qui est certainement un polymorphisme car retrouvé très fréquemment). En ce qui concerne les mutations du gène ACVRL1, nous trouvons deux mutations très majoritaires : une au niveau de l'intron 7 (mutation d'épissage c. 1048+1G>A) et l'autre au niveau de l'exon 8 (mutation faux sens p.Arg374Gln), ce qui révèle l'existence de deux effets fondateurs distincts mais géographiquement très proches (nord Deux-Sèvres).

Mots-clés : ACVRL1, Endogline, HRM.
E539. UNE NOUVELLE MUTATION FAUX-SENS ET DEUX RÉARRANGEMENTS DU GËNE FOXC2 CHEZ TROIS FAMILLES PRÉSENTANT LE SYNDROME LYMPHCEDÈME-DISTICHIASIS A.L. Fauret $(1,2)$, E. Tuleja (3), X. Jeunemaitre $(1,2)$, S. Vignes (3) (1) AP-HP, Hôpital Européen Georges-Pompidou, Département de Génétique et Centre de Référence des Maladies Vasculaires Rares, Paris, France; (2) Université Paris Descartes, Faculté de Médecine, Paris, France; (3) Hôpital Cognacq Jay, Centre de Référence des Maladies Vasculaires Rares, Paris, France

Contact : stephane.vignes@hopital-cognacq-jay.fr

Lymphoedema-distichiasis (LD) syndrome is a rare autosomal dominant disorder of the FOXC2 gene, which codes for a forkhead transcription factor. Most of the mutations described in this gene to date are deletions or insertions, suggesting a mechanism of haplo-insufficiency. In this study, three independent families with LD, presenting with both lymphoedema and distichiasis, were studied. Two microrearrangements (one 8-bp deletion and one 7-bp duplication) occurring in a GC-rich genomic region (c.893 930) known to be prone to mutations were identified. A new missense mutation, Lys $132 \mathrm{Glu}$, located in the forkhead domain, a highly conserved sequence of this transcription factor, was also identified. Mutations in this domain have been previously shown to impair FOXC2 transactivation ability. At a genetic level, this study confirms the heterogeneity of mutations responsible for LD and is consistent with a mechanism of haplo-insufficiency. At a clinical level, it reinforces the importance of genetic testing in subjects with familial lymphoedema or distichiasis, since measures can be taken at an early stage to prevent complications and to reduce the progression of lymphoedema or delay its occurrence.

IP540. OUTCOMES AND PROGNOSIS IN PATIENTS WITH TGFBR2 OR FBN1 GENE MUTATION

D. Attias $(1,2)$, C. Stheneur $(1,3,4)$, C. Roy (5), G. Collod-Béroud (6), D. Detaint (1, 2), L. Faivre (7), M.A. Delrue (8), L. Cohen (9), C. Francannet (10), C. Béroud (12), M. Claustres (12), F. Iserin (11), P. Khau Van Kien (12), D. Lacombe (13), M. Le Merrer (13), S. Lyonnet (13), S. Odent (14), H. Plauchu (15), M. Rio (13), A. Rossi (16), D. Sidi (11), C. Boileau $(1,18)$, G. Jondeau $(1,2,19)$

(1) AP-HP, Hôpital Bichat, Consultation multidisciplinaire Marfan, Paris, France : (2)AP-HP, Hôpital Bichat, Service de Cardiologie, Paris, France, and Université Denis Diderot Paris VII, Paris, France; (3) INSERM, U781, Paris, France; (4) AP-HP, Hôpital A Pare, Service de Pédiatrie, Boulogne, France, and Université Versailles-SQY, Boulogne, France ; (5) AP-HP, Hôpital Bichat, Biostatistique et Recherche Clinique, Paris, France and INSERM, U738, Paris, France ; (6) INSERM, U827, Montpellier, France and Univ Montpellier1, Montpellier, France ; (7) CHU de Dijon, Centre de Génétique, Dijon, France ; (8) CHU de Bordeaux, Service de Génétique Médicale, Bordeaux, France and Université de Bordeaux, Bordeaux, France; (9) Institut hospitalier Jacques Cartier, Unité de cardiologie pédiatrique, Massy, France ; (10) Univ Clermont1, Service de Cytogénétique Médicale. Clermont-Ferrand, France and CHU Clermont-Ferrand, UFR Médecine, France ; (II) AP-HP, Hôpital NeckerEnfants-Malades, Service de Cardiologie Pédiatrique, Paris, France and Université Paris Descartes Paris V, Paris, France ; (12) CHU Montpellier, Hôpital Arnaud-de-Villeneuve, Laboratoire de génétique moléculaire, Montpellier, France; (13) AP-HP, Hôpital Necker-Enfants-Malades, Département de Génétique médicale, Paris, France and Université Paris Descartes Paris V, Paris, France; (14) CHRU, Hôpital Pontchaillou, Rennes, France; (15) Hopital Hôtel-Dieu, Service de Génétique, Lyon, France and Université Claude Bernard, Lyon, France; (16) CHRU de Rouen, Unité de génétique Clinique, Rouen, France : (17) AP-HP, Hôpital Ambroise Paré, Laboratoire Central de Biochimie d'Hormonologie et de Génétique moléculaire, Boulogne, France: (18) INSERM, U781, Paris, France : (19) INSERM, U698, Paris, France

Contact : guillaume.jondeau@bch.aphp.fr

Background: TGFBR2 mutations were recently recognized among patients with a Marfan-like phenotype and have been associated with poor cardiovascular prognosis (early deaths, aortic dissections or early surgery for thoracic ascending aorta aneurysm). Methods : Clinical features and outcomes were analysed in a group of 71 patients (including 22 children) with a TGFBR2 mutation, collected through Marfan clinics throughout France. Patients were compared with age- and gender-matched unaffected family members $(n=50)$ and patients harbouring the FBN1 mutation (FBN1 group $n=243$ ). Systematic beta-blockade, yearly echocardiography and prophylactic aortic surgery when the aortic diameter exceeded $5.0 \mathrm{~cm}$ for adults or earlier in children were applied to patients carrying a FBN1 mutation or a TGFBR2 mutation. Results: Skeletal, ophthalmologic features and mitral involvement were less prominent in patients with TGFBR2 
mutations than in those with FBN1 mutations. The proportion of patients with aortic dilatation was similar in the TGFBR2 group and in the FBN1 group in both adults $[74 \%(35 / 47)$ vs. $76 \%(121 / 160)]$ and children $[86 \%$ (19/22) vs. $86 \%(57 / 66)]$ and aortic dilatation was very variable in both TGFBR2 and FBN1 groups. The incidence and average age of thoracic aortic surgery ( $31 \%$ vs. $27 \%$ and $35 \pm 16$ vs. $39 \pm 13$ years) were similar in the 2 groups. Surgery was performed for aortic dilatation in 12 patients and aortic dissection in 10 patients in the TGFBR2 group (vs. 43 and 23 in the FBN1 group, respectively). Both the frequency and the age of occurrence of aortic dissection were similar in the TGFBR2 and the FBN1 groups for the ascending aorta $[14 \%(10 / 71)$ vs. $10 \%(23 / 243), \mathrm{p}=0.26$; $38 \pm 12$ vs. $39 \pm 9$ years, $\mathrm{p}=0.82]$ and for the descending aorta $(3 / 71$ vs. $8 / 243, \mathrm{p}=0.72 ; 34 \pm 6$ vs. $44 \pm 10$ years, $\mathrm{p}=0.16$ ). In contrast, aortic dilatation, dissection or sudden death was the index event leading to genetic diagnosis within the family in $65 \%$ of families with TGFBR2 mutations and only $32 \%$ in families with FBN1 mutations $(p=0.002)$. When these family members were included in the survival analysis, mortality was worse in TGFBR2 families than in FBN1 families $(\mathrm{p}=0.017)$ whereas it was similar if only patients benefiting for preventive care (i.e. in whom the mutation had been identified) were included $(\mathrm{p}=0.39)$. Conclusions: The wide clinical spectrum of patients with TGFBR2 gene mutations underscores the importance of molecular biology for early diagnosis. The prognosis of a patient depends on the clinical expression of the disease and not solely on the presence of a mutation in the TGFBR2 gene.

Mots-clés : FBN1, TGFBR2, Marfan.

DP41. RENTABILITÉ DE L'ANALYSE MOLÉCULAIRE FBN1 DANS LE SYNDROME DE MARFAN COMPLET OU INCOMPLET : EXPÉRIENCE DU CENTRE DE RÉFÉRENCE

S. Funtowiez (1), V. Cusin (1), B. Grandchamp (1, 2), C. Stheneur (1-3), D. Detaint (1), G. Jondeau (1), C. Boileau (1-4), L. Gouya (1)

(1) Centre de référence Syndrome de Marfan et apparentés, Hôpital Bichat Claude-Bernard, AP-HP, Paris, France : (2) Service de biologie moléculaire, Hôpital Bichat Claude Bernard, AP-HP, Paris, France ; (3) Service de pédiatrie, CHU Ambroise Paré, Paris, France; (4) Laboratoire de biochimie,d'hormonologie et de génétique moléculaire, CHU Ambroise Paré, Paris, France

Contact : sarah.funtowiez@bch.aphp.fr

Le syndrome de Marfan (SM) est une fibrillinopathie autosomique dominante pour laquelle le diagnostic clinique est retenu selon des critères internationaux. La conférence de Berlin de 1986 retenait le diagnostic de SM devant une atteinte squelettique et d'au moins deux autres systèmes dont au moins un de manière majeure. En 1996, à Ghent, une modification des critères a permis d'affiner la clinique et d'intégrer des progrès de la génétique moléculaire. Selon les critères de Ghent, le diagnostic est retenu si 3 systèmes sont atteints dont au moins deux de manière majeure. Le SM est dâ le plus souvent à des mutations du gène FBN1 codant pour la protéine fibrilline 1. Plus rarement les gènes TGFBR1 et TGFBR2 sont mutés. Depuis 1995, le laboratoire de génétique moléculaire de l'hôpital Ambroise Paré a étudié 398 proposants du centre de référence « syndrome de Marfan et apparentés. Les critères de sélection des proposants étudiés au niveau moléculaire reposent, pour les plus anciens patients, sur la présence d'au moins un signe majeur des critères de Berlin. Ultérieurement, la sélection a été établie devant la présence d'au moins un signe majeur et de l'atteinte d'un deuxième système selon les critères de Ghent. Les mutations sont recherchées par deux approches complémentaires : i) le séquençage direct des 65 exons et régions flanquantes du gène FBN1 qui recherche les défauts moléculaires de petite taille ii) la technique de MLPA (Multiplex ligation-dependent probe amplification) qui recherche les réarrangements de grande taille sur 53 des 65 exons du gène. Une mutation du gène FBN1 a été mise en évidence chez 301 patients (75\%). Parmi les 398 proposants, 344 présentaient un diagnostic de SM selon les critères de Ghent. $280(81,4 \% ; 280 / 344)$ de ces patients, présentent une mutation délétère du gène FBN1 (170 mutations faux sens et 110 mutations caractérisées par la présence d'un codon STOP prématuré direct ou indirect). Chez 97 proposants (64 SM et 33 ne remplissant pas les critères de Ghent), aucune mutation du gène FBN1 n'a été identifiée et les gènes TGFBR2 et TGFBR1 ont été étudiés. Quatorze mutations ont alors été identifiées (10 dans le gène TGFBR2 et 4 dans le gène TGFBR1). Parmi les sujets SM, $11(3,2 \% ; 11 / 344)$ présentaient une mutation dans l'un des deux gènes ( 9 pour TGFBR2 et 2 pour TGFBR1). Ainsi, il reste 53 proposants SM $(15,4 \% ; 53 / 344)$ pour lesquels aucune altération n'a été retrouvée. Pour ces proposants nous allons : i) rechercher un remaniement majeur au niveau du promoteur et des régions non encore explorées et ii) procéder à des analyses familiales dans les familles de taille adéquate pour confirmer ou infirmer l'implication de l'un des trois gènes connus. Il est possible qu'à l'issue de ces explorations complémentaires, nous disposerons de quelques familles pour lesquelles l'implication d'un autre gène sera associée au SM, montrant ainsi l'existence d'une hétérogénéité génétique encore plus grande.

Mots-clés : syndrome de marfan, gène FBN1, hétérogénéité génétique.

P542. LA COEXISTENCE D'UN SYNDROME DE LOEYS-DIETZ DE TYPE I ET II CHEZ UN PATIENT, EXPLIQUÉE PAR LA GÉNÉTIQUE MOLÉCULAIRE

A. Plancke (1), C. Abadie (1, 2), C. Baudoin (1), P. Sarda (2, 3), M. Claustres $(1,4,5), P$. Blanchet (2), P. Khau Van Kien $(1,3)$

(1) Laboratoire de Génétique Moléculaire, CHU Montpellier, Montpellier. France; (2) Service de Génétique Médicale, CHU Montpellier, Montpellier, France ; (3) Centre de Compétence Maladies Vasculaires Rares, CHU Montpellier, Montpellier, France; (4) INSERM U827, Montpellier, France; (5) Université Montpellier 1, Montpellier, France Contact : aurelie.plancke@inserm.fr

Le syndrome de Loeys-Dietz (SLD) est caractérisé par : une transmission autosomique dominante, des mutations dans les gènes TGFBR1 et TGFBR2 et une triade : anévrismes /tortuosité artérielle diffuse, hypertélorisme et anomalie du palais ou de la luette. Le spectre des manifestations phénotypiques en rapport avec les mutations de ces gènes est très large : prédispositions au cancer (HNPCC), SLD, syndrome de Marfan de type II et prédispositions non syndromiques aux dissections de l'aorte. Le phénotype du SLD est lui même hétérogène et on distingue le SLD de type I « Marfan-like » du type II « Elhers-Danlos vasculaire (SEDv)-like ». Nous rapportons ici l'observation d'un homme de 20 ans, opéré de l'aorte ascendante à l'âge de 12 ans et présentant des signes cliniques de SEDv (2 critères majeurs : peau fine et translucide, fragilité artérielle et 3 mineurs : acrogéria, hypermobilité articulaire, pieds-bots varus-équin), de Marfan (critère majeur aortique, mineur squelettique : scoliose $>20 \%$ spondylolisthesis, pectus excavatum modéré, hypermobilité articulaire) et de SLD (état dolichoartériel diffus, fente palatine sous-muqueuse). Les antécédents familiaux sont sans particularités mais, sa mère présente de discrets signes de SEDv : peau fine et translucide, ecchymoses extensives (sans anomalies d'hémostase), récessions gingivales (greffes de gencive), hypermobilite articulaire, problèmes de cicatrisation (suites de chirurgie de hernie inguinale à 12 ans et d'une appendicectomie à 20 ans). L'étude génétique menée à partir d'un prélèvement de sang du cas index révèle la présence en trans de deux mutations non décrites à ce jour dans le même amplicon du gène TGFBR1 (exon 3 et bordures introniques) : c.400delG (prédictive d'un décalage du cadre de lecture) et c.344-4delT (ou IVS2-4delT). L'étude du prélèvement des parents montre que la mutation c.400delG est survenue de novo sur un chromosome paternel (sauf mosaïcisme germinal) alors que la mutation IVS2-4delT est héritée de la mère. L'étude d'une culture de fibroblastes cutanés obtenue chez le cas index permettant l'analyse des ARN totaux révèle trois populations de transcrits TGFBR1 : 1-mutation c.400delG, 2 - transcrits normaux et 3 - insertion de 11 nucleotides introniques en rapport avec l'activation par la mutation IVS2-4delT d'un site cryptique accepteur d'épissage. Le patient présente donc une hétérozygotie composite, avec un allèle maternel hypomorphe (anomalie partielle d'épissage) et un allèle nul de novo. Dans cette famille, l'allèle IVS2-4delT confere manifestement un phénotype SLD type II «SEDv-like » alors que l'allèle c.400delG est associé à un phénotype SLD type I « Marfan-like ». Il s'agit à notre connaissance de la première corrélation génotype/phénotype pour les mutations de TGFBR1.

Mots-clés : Loeys-Dietz, hétérozygotie composite, corrélation génotype/phénotype.

IP543. UNE MUTATION R611Q DE LA SOUS-UNITÉ MED23 DU COMPLEXE MÉDIATEUR EST ASSOCIÉE À UNE FORME AUTOSOMIQUE RÉCESSIVE DE RETARD MENTAL NON SYNDROMIQUE

S. Boissel*, M. Zarhrate*, M. Rio, A. Munnich, L. Colleaux

INSERM U78I and Département de Génétique, Université Paris Descartes, Hôpital Necker-Enfants Malades, Paris, France

* Ces deux auteurs ont contribué de façon équivalente au travail.

Contact : sarah.boissel@inserm.fr

Le retard mental (RM) est défini comme « un fonctionnement intellectuel significativement inférieur à la moyenne associé à des limitations des fonctions adaptatives » (DSM-IV). $25 \%$ des cas de retard mental non syndromique (RMNS), où le RM est le seul signe clinique observé, s'expliquent par un mode de transmission autosomique récessif (AR). Néanmoins, l'extrême hétérogénéité des formes récessives autosomiques et la taille souvent trop restreinte des fratries a longtemps freiné l'identification des gènes responsables de RM récessifs autosomiques. Seuls 5 gènes de RMNS AR ont été identifiés à ce jour. Nous avons eu l'opportunité d'étudier une 
famille consanguine algérienne dans laquelle 5 enfants atteints présentent un RMNS. Mettant à profit la stratégie de cartographie par autozygotie, nous avons mis en évidence une région unique d'homozygotie de $6,5 \mathrm{Mb}$ en 6q22.31-q23.2, avec un LOD score maximum de 4,16 au locus D6S3136. Cette région contient 44 gènes connus. Nous avons identifié une variation homozygote dans le gène MED23 (p.R611Q), qui co-ségrége avec la maladie et n'a pas été retrouvée chez 608 chromosomes contrôles (dont 242 chromosomes de la même origine ethnique), suggérant qu'elle est vraisemblablement à l'origine du RM. MED23 code une des sous-unités d'un large complexe multiprotéique : le Médiateur (MED). Ce complexe est un élément clef de la machinerie générale de transcription qui sert d'interface dynamique entre les facteurs de transcription spécifiques et l'ARN polymérase II (Ryu et al., Nature, 1999). De façon très intéressante, des mutations faux sens de la sous unité MED12 sont associées aux syndromes d'Opitz-Kaveggia (Risheg et al., Nature genetics, 2007) et de Lujan (Schwartz et al., Journal of Medical genetics, 2007), qui sont deux syndromes de RM liés à l'X. Des expériences de RT-PCR sur des ARNs de tissus adultes et fætaux montrent une expression ubiquitaire de MED23. Par ailleurs, des études de RT-PCR quantitative réalisées dans des cellules de patients, suggèrent une anomalie d'expression de certains des gènes régulés par le complexe MED. Enfin, des études par immunoprécipitation de chromatine sont maintenant en cours pour tenter de corréler ces anomalies à un éventuel défaut d'assemblage des différents partenaires de la machinerie transcriptionnelle. En conclusion, nos résultats confirment l'efficacité de la cartographie par autozygotie pour identifier de nouveaux gènes responsables de pathologies autosomiques récessives rares. Ils apportent également un nouvel argument en faveur du rôle majeur du complexe MED dans l'étiologie des retards mentaux. Enfin, les analyses en cours devraient nous permettre de mieux caractériser la fonction de MED23 au cours du développement du système nerveux central.

Mots-clés : retard mental, complexe mediator, transcription. D544. MALADIES GÉNÉTIQUES EN TUNISIE : UNE REVUE
SYSTÉMATIQUE

L. Romdhane, I. Manai, S. Romdhane, S. Abdelhak

Investigation Moléculaire des Maladies Orphelines d'Origine Génétique, Institut Pasteur de Tunis, Tunisie

Contact : sonia.abdelhak@hexabyte.tn

La Tunisie est un pays Nord Africain situé à un carrefour entre l'Afrique et l'Europe. Les données concernant les maladies génétiques dans les pays en voie de développement, tel que la Tunisie, sont peu disponibles bien que les pathologies héréditaires y sont considérées comme un réel problème de santé publique à cause du taux élevé de la consanguinité qui peut atteindre $60 \%$. L'objectif de cette présente étude est d'évaluer l'ampleur des maladies génétiques affectant la population tunisienne à travers une revue de la littérature scientifique. Une recherche dans les textes d'OMIM a été effectuée. Une revue systématique des publications de PubMed en utilisant les mots-clés « genetic disease Tunisia » et « Tunisian patient " a été aussi réalisée. Les données de la « littérature grise » ont été récupérées à partir des rapports de meetings nationaux et internationaux, journaux locaux, thèses de médecine et de biologie ainsi que de monographes. Les rapports comprenant les données épidémiologiques, cliniques, génétiques et moléculaires ont été sélectionnés. Les données concernant les maladies génétiques affectant la population tunisienne concernent des patients vivant en Tunisie et à l'étranger. Une liste non exhaustive de 251 de désordres génétiques a été identifiée. Parmi eux, $66,93 \%$ sont autosomiques récessifs, $21,51 \%$ autosomiques dominants, $5,18 \%$ liés au chromosome $\mathrm{X}, 0,4 \%$ liés au chromosome $\mathrm{Y}, 0,8 \%$ à transmission mitochondriale, $2 \%$ à transmission mixte, $2,4 \%$ sporadique, et $0,8 \%$ de mode de transmission inconnue. Cinquante cinq pour cent de ces maladies génétiques ont une étiologie moléculaire connue et sont due à au moins une mutation. Un effet fondateur a été noté dans $7,17 \%$ des maladies dont $50 \%$ sont spécifiques à la population Tunisienne, le reste étant communs à d'autres populations nord africaines. Pour plusieurs cas, l'hétérogénéité est responsable pour leur fréquence élevée. L'hétérogénéité allélique est aussi marquée et conduit à l'expression du même phénotype morbide. Étant donné les taux élevés de consanguinité et endogamie en Tunisie combinés avec un effet fondateur, plusieurs désordres génétiques ont émergé, ceux qui sont transmis selon le mode récessifs comptant pour la majorité. Actuellement en Tunisie, il existe plusieurs études sur la prévention de l'handicap à travers les réseaux pluridisciplinaires. Les programmes d'éducation sur la santé tôt pendant le cursus scolaire semble être un excellent moyen de prévention de telles pathologies pour un pays en voie de développement comme la Tunisie. Pour des raisons géographiques, socioculturelles et historiques, notre étude a un impact au niveau régional, étant donné que la structure de la population tunisienne est très similaire à celles des pays voisins.
- 545 . DÉVELOPPEMENT DU DIAGNOSTIC MOLÉCULAIRE POUR LES MALADIES GÉNÉTIQUES RARES EN TUNISIE

R. Kefi-Ben Atig pour l'Unité de recherche sur les maladies orphelines d'origine génétique (contribution égale par ordre alphabétique : S. Abdelhak, A. Amouri, A. Abid, I. Arfa, M. Bchetnia, A. Sabrine Ben Brick, N. Ben Halim, M. Ben Rekaya, F. Ben Rhouma, C. Charfeddine, S. Chakroun, F. Cherif, L. Chérif-Ben Abdallah, W. Chérif, I. Chouchane, I. Dorboz, K. Lasram, O. Messaoud, H. Messai, S. Nouira, F. Ouechtati, H. Ouragini, L. Romdhane, F. Talmoudi, M. Majdi Zorgati), M. Mokni, S. Boubaker, M.F. Ben Dridi, L. Elmatri, F. Warda, K. Monastiri, H. Ben Abid, N. Miladi, S. Kachboura, N. Kaabachi, F. Amri, A. Ayadi, M. Tahar Sfar

Exploration Moléculaire des Maladies Orphelines d'origine GénétiqueInstitut Pasteur de Tunis, Tunisie

Contact : rym.kefi@pasteur.rns.tn

En Tunisie, comme dans tous les pays nord africains, la fréquence des maladies génétiques est relativement élevée, notamment pour les maladies à transmission autosomique récessive, à cause de la consanguinité et de l'endogamie. Au cours de ces dernières années, nous avons contribué à la caractérisation moléculaire de plusieurs maladies génétiques rares telles que l'anémie de Fanconi, les BCGites, I'anémie mégaloblastique, la kératodermie palmo-plantaire et la granulomatose septique chronique. Une stratégie standardisée a été adoptée. Dans un premier temps, le génotype des patients et de leurs familles est déterminé par des marqueurs moléculaires polymorphes de type microsatellites, flanquant les gènes candidats connus dans la littérature. L'implication ou non du ou des gènes candidats est mise en évidence par homozygotie par descendance. Dans le cas d'une liaison génétique, la recherche des mutations est réalisée par séquençage direct de la région codante et de la jonction exon/ intron du gène d'intérêt. L'étude de ces affections a montré une hétérogénéité clinique et génétique reflétant une grande richesse du patrimoine génétique de la population tunisienne. Ce haut degré de polymorphisme est dû aux flux migratoires multidirectionnels qu'a connu l'Afrique du Nord tout au long de son histoire. La valorisation de ces résultats de recherche s'est traduite par la mise en place du diagnostic moléculaire pour plusieurs maladies rares, handicapantes sur le plan physique et social, telles que la maladie de Gaucher, les glycogénoses type la et type III et les maladies cassantes de l'ADN $\mathrm{Ce}$ qui a permis une meilleure prise en charge des patients et de leurs familles.

\section{Pharmaco-génétique}

DP546. EST-IL PERTINENT D'INTEGRER LE TEST GÉNÉTIQUE DE KRAS À L'ARBRE DÉCISIONNEL THÉRAPEUTIQUE DU CANCER COLORECTAL MÉTASTATIQUE ?

S. Küry (1, 2), C. Bossard (2), F. Airaud (1), I. Maury (1), P. Jamet (3), H. Senellart (4), T. Matysiak-Budnik (3), J.F. Mosnier (2), S. Bézieau (1, 2)

(1) CHU de Nantes, pôle de Biologie, service de Génétique Médicale, Nantes, France; (2) Université de Nantes, Faculté de Médecine, EA 427.3 Biométadys, Nantes, France; (3) CHU de Nantes, Institut des Maladies de l'Appareil Digestif, Service d'Hépato-Gastroentérologie, Nantes, France; (4) Centre de Lutte Contre le Cancer Nantes Atlantique René Gauducheau, Nantes, France

Contact : sebastien.kury@chu-nantes.fr

Introduction : Des études récentes ont montré que les patients atteints d'un cancer colorectal (CCR) métastatique dénué de mutation du gène KRAS pouvaient bénéficier d'un traitement associant à une chimiothérapie conventionnelle un anticorps monoclonal dirigé contre le récepteur à l'EGF (Epidermal Growth Factor). Or, il apparaît que seuls 20 à $50 \%$ des patients non mutés présentent une réponse objective à ce traitement. L'hétérogénéité tumorale pourrait être l'une des raisons de cette " résistance » thérapeutique. Pour vérifier cette hypothèse, nous avons comparé les statuts mutationnels des gènes KRAS et BRAF entre la tumeur primitive et les métastases correspondantes, synchrones et/ou métachrones, de patients atteints de CCR métastatique. Méthode : Le statut mutationnel des gènes KRAS (codons 12 et 13) et BRAF (mutation V600E) a été analysé rétrospectivement dans les tumeurs primitives $(n=24)$, les métastases viscérales synchrones et/ou métachrones $(n=33)$ et les récidives locales $(n=1)$ de 20 patients atteints d'un CCR métastatique et traités par un anticorps antiEGFR. Pour chaque patient, le nombre de métastases analysables était compris entre 1 et 4 . Au total, 17 appariements entre tumeur primitive et métastase(s) ou récidive ont pu être étudiés. L'analyse mutationnelle a été réalisée par séquençage direct des amplicons PCR d'ADN génomique extrait de coupes paraffinées. La zone d'intérêt était systématiquement 